\title{
Purinergic Signalling: Therapeutic Developments
}

\author{
Geoffrey Burnstock ${ }^{1,2 *}$ \\ ${ }^{1}$ Autonomic Neuroscience Centre, University College Medical School, London, United Kingdom, ${ }^{2}$ Department of \\ Pharmacology and Therapeutics, The University of Melbourne, Melbourne, VIC, Australia
}

\section{OPEN ACCESS}

Edited by:

Kenneth A. Jacobson,

National Institutes of Health (NIH),

United States

Reviewed by:

Stanko S. Stojilkovic,

National Institutes of Health (NIH)

United States

Rennolds S. Ostrom

Chapman University, United States

${ }^{*}$ Correspondence:

Geoffrey Burnstock

g.burnstock@ucl.ac.uk

Specialty section:

This article was submitted to

Experimental Pharmacology and Drug

Discovery,

a section of the journal

Frontiers in Pharmacology

Received: 07 August 2017 Accepted: 05 September 2017 Published: 25 September 2017

Citation:

Burnstock G (2017) Purinergic Signalling: Therapeutic Developments.

Front. Pharmacol. 8:661. doi: 10.3389/fphar.2017.00661
Purinergic signalling, i.e., the role of nucleotides as extracellular signalling molecules, was proposed in 1972. However, this concept was not well accepted until the early 1990's when receptor subtypes for purines and pyrimidines were cloned and characterised, which includes four subtypes of the P1 (adenosine) receptor, seven subtypes of P2X ion channel receptors and 8 subtypes of the P2Y G protein-coupled receptor. Early studies were largely concerned with the physiology, pharmacology and biochemistry of purinergic signalling. More recently, the focus has been on the pathophysiology and therapeutic potential. There was early recognition of the use of $\mathrm{P} 1$ receptor agonists for the treatment of supraventricular tachycardia and $\mathrm{A}_{2 \mathrm{~A}}$ receptor antagonists are promising for the treatment of Parkinson's disease. Clopidogrel, a $\mathrm{P}_{2} \mathrm{Y}_{12}$ antagonist, is widely used for the treatment of thrombosis and stroke, blocking $\mathrm{P}_{2} \mathrm{Y}_{12}$ receptor-mediated platelet aggregation. Diquafosol, a long acting $\mathrm{P} 2 \mathrm{Y}_{2}$ receptor agonist, is being used for the treatment of dry eye. P2X3 receptor antagonists have been developed that are orally bioavailable and stable in vivo and are currently in clinical trials for the treatment of chronic cough, bladder incontinence, visceral pain and hypertension. Antagonists to P2X7 receptors are being investigated for the treatment of inflammatory disorders, including neurodegenerative diseases. Other investigations are in progress for the use of purinergic agents for the treatment of osteoporosis, myocardial infarction, irritable bowel syndrome, epilepsy, atherosclerosis, depression, autism, diabetes, and cancer.

\section{Keywords: ATP, adenosine, CNS diseases, peripheral diseases, infection, inflammation}

\section{INTRODUCTION}

Purinergic signalling, i.e., nucleotides as extracellular signalling molecules, was proposed in 1972 (Burnstock, 1972). However, this concept was not well accepted until the 1990's when receptor subtypes for purines and pyrimidines were cloned and characterised, which includes four subtypes of the P1 (adenosine) receptor, seven subtypes of P2X ion channel receptors, and eight subtypes of the P2Y G protein-coupled receptor (Ralevic and Burnstock, 1998). Early studies were largely concerned with the physiology, pharmacology and biochemistry of purinergic signalling (Burnstock, 2007). Adenosine $5^{\prime}$-triphosphate (ATP) is a cotransmitter with classical transmitters in both the peripheral and central nervous systems. In addition, purines are powerful extracellular messengers to non-neuronal cells, including secretory, exocrine and endocrine, endothelial, immune, musculo-skeletal and inflammatory cells (Burnstock and Knight, 2004). Purinergic signalling is rapid in neurotransmission, neuromodulation and in secretion, but is also long-term in proliferation, differentiation, migration and death in development and regeneration (Burnstock, 2016f). 
More recently, the focus has been on the pathophysiology and therapeutic potential of both P1 (Chen et al., 2013; de Lera Ruiz et al., 2014; Layland et al., 2014; Liu and Xia, 2015; Borea et al., 2016) and P2 (Burnstock and Kennedy, 2011; Bartlett et al., 2014; Ford et al., 2015; Burnstock, 2016e) receptors. Reviews focussed on different aspects of purinergic pathophysiology are also available, including inflammatory and immune disorders (Arulkumaran et al., 2011; Junger, 2011; Hansson et al., 2016); cancer (Burnstock and Di Virgilio, 2013; Di Virgilio and Adinolfi, 2017); gout and fibrosis (Gicquel et al., 2017); P2X7 receptors (R) as therapeutic targets (Romagnoli et al., 2008); medicinal chemistry of purinoceptors (Jacobson and Muller, 2016); pain (Burnstock and Sawynok, 2010; Alves et al., 2013; Kuan and Shyu, 2016; Sawynok, 2016) and adenosine kinase inhibitors (Kowaluk and Jarvis, 2000). A number of purine-related compounds have been patented. Therapeutic developments for disorders of different systems in the body will now follow. Reviews concerned with the early literature will be quoted, so the focus of this review will be concerned largely with the most recent findings.

\section{DISORDERS OF THE CENTRAL NERVOUS SYSTEM (CNS)}

Investigations into purinergic signalling and its roles in disorders of the CNS have been reported, for instance following surgery, stroke, accidents and ischemia, neurodegenerative diseases (such as Parkinson's, Alzheimer's and Huntington's diseases), multiple sclerosis (MS), amyotrophic lateral sclerosis (ALS), epilepsy and neuropsychiatric disorders (including schizophrenia, depression and anxiety). Reviews covering this topic are available (Burnstock, 2008b; Burnstock et al., 2011), including the recent attention to the development of centrally penetrant P2X7R antagonists for the treatment of CNS disorders (Burnstock and Verkhratsky, 2012; Puchalowicz et al., 2014; Sperlagh and Illes, 2014; Burnstock, 2015b; Cisneros-Mejorado et al., 2015b).

\section{Neurodegenerative Diseases}

Neurodegeneration in the CNS is associated with inflammation and damage to both neurons and glia (Rama Rao and Kielian, 2015). Reviews are available that include coverage of early papers concerned with neurodegenerative diseases (Puchalowicz et al., 2014; Santiago et al., 2014; Fasullo and Endres, 2015; Förster and Reiser, 2015; Burnstock, 2016a; Fumagalli et al., 2016). Recent attention has been directed toward the use of P2X7R antagonists for the treatment of neurodegenerative diseases (Metzger et al., 2017). However, adenosine acting via $A_{2 A} R$ has also been claimed as a promising therapeutic agent for the prevention and treatment of neurodegenerative diseases (Cunha, 2016; Harmse et al., 2016; Olatunji et al., 2016).

\section{Alzheimer's Disease (AD)}

There is progressive cognitive impairment in $\mathrm{AD}$, with prominent deficits in short term memory. The potential of purinergic drugs for the treatment of $\mathrm{AD}$ has attracted much interest in recent years. Previous studies have led to the proposal that both P2X7R and $\mathrm{P} 2 \mathrm{Y}_{4} \mathrm{R}$ antagonists are potential therapeutic targets for the treatment of AD (Erb et al., 2015; Miras-Portugal et al., 2015; Woods et al., 2016). It has been suggested that the blockade of $\mathrm{P}_{2} \mathrm{Y}_{1} \mathrm{R}$ may have therapeutic potential against cognitive disturbances in AD (Guzman and Gerevich, 2016). $\beta$-Amyloid increased release of ATP, which potentiated excitatory synaptic activity via P2XR, effects that were blocked by P2X antagonists (Sáez-Orellana et al., 2016). The glycosylphosphatidylinositolanchored prion protein binds to and modulates the expression of P2X4R, which may be involved in AD (Carneiro et al., 2016). Recently, a potential role has been proposed for ATP-sensitive potassium channel $\left(\mathrm{K}_{\mathrm{ATP}}\right)$ modulation as a therapeutic strategy against AD (Salgado-Puga et al., 2017).

Adenosine $\mathrm{A}_{3} \mathrm{R}$ agonists suppress amyloid- $\beta$ protein precursor internalisation and amyloid- $\beta$ generation (Li S. et al., 2015). Hippocampal adenosine $A_{2 A} R$ up-regulation is necessary to trigger memory dysfunction in $\mathrm{AD}$ (Cunha, 2015).

\section{Parkinson's Disease (PD)}

The involvement of adenosine $\mathrm{A}_{2 \mathrm{~A}} \mathrm{R}$ in $\mathrm{PD}$ and their interactions with dopamine receptors has attracted most attention (see Jenner, 2014). $A_{2 \mathrm{~A}} \mathrm{R}$ antagonists have been proposed for the treatment of PD (Jenner, 2014; Mori, 2014; Pinna, 2014; Navarro et al., 2016). $\mathrm{K}_{\mathrm{ATP}}$ (Dragicevic et al., 2015) and P2X1R (Gan et al., 2015; Navarro et al., 2016; Yang Z. et al., 2016) in PD have also been reported, perhaps indicating new therapeutic targets. Clinical trials istradefylline, an $\mathrm{A}_{2 \mathrm{~A}} \mathrm{R}$ antagonist, have taken place and it may have a beneficial effect in conjunction with commonly used anti-Parkinson's therapies (Tao and Liang, 2015; Uchida et al., 2015; Vorovenci and Antonini, 2015), although in a later study, istradefylline was shown to enhance amyloid- $\beta$ generation and $\gamma$-secretase activity (Lu et al., 2016). Oligomerisation kinetics of $\mathrm{A}_{2 \mathrm{~A}}$ and dopamine $\mathrm{D}_{2} \mathrm{R}$ have important implications for PD (Casadó-Anguera et al., 2016; Ferré et al., 2016; GuixàGonzález et al., 2016). Clinical trials for $A_{2 A}$ antagonists for PD are assessed in a review (Navarro et al., 2016). $\mathrm{A}_{2 \mathrm{~A}} \mathrm{R}$ inhibition stopped rotenone-induced motor impairment in a rat model of PD (Fathalla et al., 2016). $A_{1}$ as well as $A_{2 A} R$ antagonists were recommended as promising candidates for treatment of $\mathrm{PD}$ in a recent paper (Essawy et al., 2017).

P2X7R antagonists have also been implicated in this disease (Jörg et al., 2014; Wang et al., 2017). A P2X7R antagonist, brilliant blue G, was recently shown to be protective in a lipopolysaccharide (LPS) animal model of PD (Wang et al., 2017).

\section{Huntington's Disease (HD)}

Earlier studies reported that $\mathrm{A}_{2 \mathrm{~A}} \mathrm{R}$ agonists could be therapeutically useful for $\mathrm{HD}$ and that P2X7R antagonists inhibited neuronal apoptosis and attenuated body weight loss and motor co-ordination deficit in HD patients. Adenosine $A_{2 A} R$ inhibition reversed working memory deficits of $H D$ at early stages models and it was proposed that $A_{2 A} R$ antagonists may therapeutically reverse the cognitive deficits in HD patients (Li W. et al., 2015). In earlier publications, $A_{1} R$ agonists were suggested to be therapeutic targets (Ferrante et al., 2014; Lee and Chern, 2014). It has been claimed that $\mathrm{K}_{\mathrm{ATP}}$ channels may be potential targets for treatment of HD (Gupta and Sharma, 2014). 
Inhibition of equilibrative nucleoside transporter 1 enhances the adenosine level and may be a potential therapeutic approach for treating HD (Kao et al., 2017).

\section{Amyotrophic Lateral Sclerosis}

Involvement of $\mathrm{A}_{2 \mathrm{~A}} \mathrm{R}, \mathrm{P} 2 \mathrm{X} 4 \mathrm{R}$ and $\mathrm{P} 2 \mathrm{X} 7 \mathrm{R}$ in ALS has been reported (Volonté et al., 2016). $\mathrm{A}_{1} \mathrm{R}$ have also been implicated in ALS (Nascimento et al., 2015). Preconditioning with latrepirdine, an adenosine $5^{\prime}$-monophosphate (AMP)-activated protein kinase activator, was beneficial in the SOD1 mouse model of ALS (Coughlan et al., 2015). $\mathrm{A}_{2 \mathrm{~A}} \mathrm{R}$ activation facilitated neuromuscular transmission in SOD1 mice during the pre-symptomatic but not symptomatic phase of the disease (Nascimento et al., 2014). Pharmacological inhibition of $\mathrm{A}_{2 \mathrm{~A}} \mathrm{R}$ protects against degeneration of spinal motor neurons in the mouse SOD1G93A ALS model (Ng et al., 2015).

P2X7R expression was significantly reduced, leading to $\mathrm{Ca}^{2+}$ disturbances in peripheral blood mononuclear cells in ALS patients (Liu J. et al., 2016). Low concentrations of endogenous ATP acting on P2X7R induced motor neuron death (Gandelman et al., 2013). Spinal cord pathology was reduced by P2X7R inhibitors in the mouse SOD1 ALS model (Apolloni et al., 2014).

\section{Multiple Sclerosis}

P2X7R and the P2YR-like GPR17 are involved in MS (Plemel et al., 2014; Burnstock, 2015c; Ou et al., 2016). There is upregulation of ecto- $5^{\prime}$-nucleotidase (CD73) in experimental autoimmune encephalomyelitis, which is an MS animal model (Lavrnja et al., 2015). Genetic variants in P2X7R affect susceptibility to MS (Gu et al., 2015; Sadovnick et al., 2017).

\section{Brain Injury, Neuroprotection, and Neuroregeneration \\ Brain Injury}

The importance of inflammation in the responses to brain injury has been reviewed and the involvement of P1R and P2R (Fiebich et al., 2014; Beamer et al., 2016) and of P2X7R in particular (Burnstock, 2016b; Giuliani A.L. et al., 2017). The roles of purinergic signalling in neurodegeneration as a consequence of brain injury, neuroprotection and neuroregeneration have been discussed in reviews ( $\mathrm{Hu}$ et al., 2014; Burnstock, 2016a). Ectonucleotidase activities and nucleotide levels in serum are altered by brain stab injury (Parabucki et al., 2014; Laketa et al., 2015). P2X7R antagonists appear to be effective as a treatment for radiation injury (Xu et al., 2015). Astrocytic p-connexin 43 stimulates neuronic autophagy through P2X7R activation in the hippocampus, resulting in brain injury-induced cognitive deficit repair (Sun L. et al., 2015). P2X7R antagonists have been implicated as a novel target to prevent secondary neurological injury after traumatic brain injury (Kimbler et al., 2012) and after spinal cord injury (Peng et al., 2009). P2X7R antagonists could be therapeutically effective to treat stroke patients (Kuan et al., 2015). P2X7R stimulation in acute ischaemic stroke is beneficial by restricting early oedema formation and perhaps by modulating responses of glia (Kaiser et al., 2016). Hypoxic-ischaemic brain injury increases intracellular $\mathrm{Ca}^{2+}$ in oligodendrocytes, which is partly mediated by P2X7R (Fern et al., 2014). P2X7R antagonists may provide a new target for the treatment of cerebral ischaemia (Bai and Li, 2013; Yu Q. et al., 2013) and for the prevention of ischaemic damage to oligodendrocytes (Domercq et al., 2010). P2X7 antagonists or inhibition of pannexin-1 channels reduced brain damage following ischaemia (Cisneros-Mejorado et al., 2015a; Mahi et al., 2015).

Activation of upregulated $\mathrm{P}_{2} \mathrm{Y}_{1} \mathrm{R}$ results in neuroblast migration to sites of brain damage (Cao et al., 2015). Microglial $\mathrm{P} 2 \mathrm{Y}_{12} \mathrm{R}$ activation follows neuronal injury (Swiatkowski et al., 2016). $A_{2 A} R$ activation was suggested for the treatment of brain injury and subsequent neuroinflammation (Dai and Zhou, 2011). $A_{2 A} R$ on cells derived from bone marrow modulate white matter lesions following chronic cerebral hypoperfusion (Ran et al., 2015). Diadenosine tetraphosphate $\left(\mathrm{Ap}_{4} \mathrm{~A}\right)$ may be a good candidate for traumatic spinal cord injury treatment (Reigada et al., 2017). Adenosine kinase facilitated astrogliosis after traumatic brain injury and its inhibition in reactive astrocytes ameliorated astrogliosis-induced cell death (Jin et al., 2016).

ATP and adenosine concentrations in the brain were significantly raised during brain ischaemia to stimulate $\mathrm{P} 1 \mathrm{R}$ and P2R (Cisneros-Mejorado et al., 2015b; Pedata et al., 2016). P2X4R expressed by microglia are involved in postischaemic brain inflammation (Cheng et al., 2014). P2X4R are required for neuroprotection via ischemic preconditioning (Ozaki et al., 2016). Neuronal $\mathrm{K}_{\mathrm{ATP}}$ channels play a role in hypoxic preconditioning where they decrease neonatal hypoxicischaemic brain injury; it has been proposed that openers of $\mathrm{K}_{\mathrm{ATP}}$ channels may prove to be therapeutically beneficial (Sun H.S. et al., 2015). Intranasal administration of guanosine reduced ischaemic brain damage in rats (Ramos et al., 2016). Ambiguously, both $\mathrm{A}_{2 \mathrm{~A}} \mathrm{R}$ agonists and antagonists may protect against ischaemic brain injury (Pedata et al., 2014). $A_{1} R$ contribute to immune responses following neonatal hypoxic ischaemic brain injury (Winerdal et al., 2016). A valuable review of the involvement of P1R, P2XR and P2YR in brain ischemia is available (Pedata et al., 2016).

\section{Neuroprotection and Neuroregeneration}

This topic is explored in recent reviews (Rodrigues et al., 2015; Burnstock, 2016a; Illes et al., 2016). Inhibition of P2XR and P2YR as well as activation of P1R following ATP breakdown to adenosine released from CNS cells, have been shown to be neuroprotective. Activation of the pannexin 1/P2X7R complex contribute to the neuroprotection that occurs after ischaemic pre- and post-conditioning (Mahi et al., 2015). Docosahexaenoic acid in the diet is thought to be a purinergic modulator via P2XR where it protects against neurodegenerative diseases (Molz et al., 2015). Neuroprotection mediated by microglia is associated with $\mathrm{P} 2 \mathrm{X} 7 \mathrm{R}$ activation and release of tumour necrosis factor- $\alpha$ (Masuch et al., 2016). Blockade of P2X7R provides neuroprotection against stroke, traumatic brain injury and subarachnoid haemorrhage (Zhao H. et al., 2016). P2X7R antagonists improve recovery after spinal cord injury (Wang et al., 2004). From a recent study on $\mathrm{P}_{2} \mathrm{Y}_{2} \mathrm{R}$ knockout (KO) mice and murine cell lines, it was concluded that $\mathrm{P}_{2} \mathrm{Y}_{2} \mathrm{R}$ play a neuroprotective role in neurological disorders, especially $\mathrm{AD}$ (de Diego-Garcia et al., 2017). Neuroprotection is claimed to 
be mediated by $\mathrm{P}_{2} \mathrm{Y}_{13}$ nucleotide receptors in neurons (PérezSen et al., 2015). Activation of $\mathrm{P}_{2} \mathrm{Y}_{2} \mathrm{R}$ evokes regeneration of glial cells and nerves and the P2YR-like GPR17 evokes oligodendrocyte regeneration. Neural stem cell activation leads to neuroregeneration, probably via P2X4R and P2X7R. Reviews focused on the roles played by P2X4R and P2X7R (MirasPortugal et al., 2015) and $\mathrm{A}_{2 \mathrm{~A}} \mathrm{R}$ (Ribeiro et al., 2016) in neurodegeneration and neuroprotection have been published.

\section{Psychiatric Disorders}

Several antipsychotic drugs (e.g., chlorpromazine, fluspirilene, and haloperidol) were found to antagonise responses mediated by P2XR. These antipsychotic drugs act therapeutically by inhibiting dopaminergic hyperactivity by suppressing P2X-mediated effects (see Burnstock, 2015b). Brain penetrant P2X7R antagonists are being developed as drug targets for psychiatric diseases (Bhattacharya and Biber, 2016). Reviews about purinergic signalling in psychiatric disorders, such as addiction, depression, schizophrenia, bipolar disorder and autism, have been previously published (Yamada et al., 2014; Lindberg et al., 2015; Krügel, 2016). The possibility has been raised that P1R agonists might be beneficial in the therapy of psychiatric disorders (Cieslak et al., 2016).

\section{Schizophrenia}

Adenosine neuromodulation in schizophrenia has received the most attention (see Rial et al., 2014; Ciruela et al., 2015; Turcin et al., 2016). Deletion of $A_{2 A} R$ from astrocytes interferes with glutamate homeostasis resulting in cognitive and psychomotor impairment in schizophrenia (Matos et al., 2015). It has been suggested that P2X7R and pannexin 1 channels are involved (see Burnstock and Verkhratsky, 2012; Avendano et al., 2015).

\section{Bipolar Disorder}

P2X7R, mediating neuroinflammation via microglial activity, contribute to bipolar disorder (see Gubert et al., 2013, 2016; Barron et al., 2014). Serum concentrations of uric acid increase in different clinical phases of bipolar disorder (Albert et al., 2015) and in those patients treated with lithium (Muti et al., 2015). The purinergic system may become dysregulated during manic episodes and it has been proposed that raised uric acid levels could be a useful indicator of bipolar disorder manic phases (Bartoli et al., 2017a). Purinergic modulators that reduce levels of uric acid may be therapeutically beneficial (Bartoli et al., 2017b). However, a decrease in serum adenosine levels in bipolar disorder patients was reported (Gubert et al., 2016).

\section{Depression and Anxiety}

Reviews describing the roles of both P1 $\left(\mathrm{A}_{1}\right.$ and $\left.\mathrm{A}_{2 \mathrm{~A}}\right)$ and P2X7R in mood disorders are available (Yamada et al., 2014; Ortiz et al., 2015). Antidepressant effects of P2X7 antagonists have been reported (Pereira et al., 2013; Zhang K. et al., 2016). P2X2R in the medial prefrontal cortex mediate the antidepressant-like actions of ATP released from astrocytes (Cao et al., 2013). Brilliant blue G, a P2X7R antagonist, had antidepressant and antiinflammatory actions in mice after LPS administration (Ma et al., 2014). The P2X7R antagonist, A-804598, affected neuroimmune and behavioural features of stress (Catanzaro et al., 2014). Stressrelated mood disorders activate the inflammasome via release of ATP and stimulation of P2X7R (Iwata et al., 2016). Co-expression of wild-type P2X7R with the polymorphism variant Gln460Arg alters receptor function associated with mood disorders (AprileGarcia et al., 2016). Increased $\mathrm{K}_{\mathrm{ATP}}$ channel activity due to a mutation led to reduced anxiety in mice (Lahmann et al., 2014). $K_{\text {ATP }}$ channels are involved in the pathogenesis of depression and may be a therapeutic target for this disorder (Fan et al., 2016). Altered levels of several ATP-dependent chromatin remodelling factors may be linked to high trait anxiety (Wille et al., 2016).

$\mathrm{A}_{2 \mathrm{~A}} \mathrm{R}$ antagonists were reported to have antidepressant activity (Yamada et al., 2014). Creatine and ketamine had antidepressant effects, probably mediated by activation of $A_{1} R$ and $A_{2 A} R$ (Cunha et al., 2015). Caffeine, acting as an $A_{2 A} R$ antagonist, prevented depression triggered by chronic stress (Kaster et al., 2015; Dziubina et al., 2016). Striatal and extrastriatal $\mathrm{A}_{2 \mathrm{~A}} \mathrm{R}$ in the forebrain regulate fear responses in mice (Wei et al., 2014). $A_{2 A} R$ mediated increased interleukin (IL)- $1 \beta$ in the brain contributed to anxiety (Chiu et al., 2014). Fear and anxiety in a mouse model of post-traumatic stress disorder were alleviated by administration of a derivative of adenosine, WS0701 (Huang et al., 2014). $A_{1} R$ agonists have been used to treat anxiety, but have troublesome side-effects so positive allosteric modulators have been developed as potent anxiolytic agents (Vincenzi et al., 2016). An antidepressant-like effect of inosine in mice has been reported (Gonçalves et al., 2017).

\section{Autism}

Adenosine in addition to suramin, a non-selective ATP antagonist, are reported to improve behaviour in autistic individuals (Masino et al., 2013; Naviaux et al., 2014, 2015; Hamidpour et al., 2016).

\section{Addiction}

Targeting $\mathrm{A}_{2 \mathrm{~A}} \mathrm{R}$ may offer strategies for combating drug addiction. Striatopallidal $\mathrm{A}_{2 \mathrm{~A}} \mathrm{R}$ signalling in the dorsomedial striatum has been suggested as a therapeutic target in drug addiction by reducing habit formation (Li et al., 2016). Caffeine potentiates the addictive effects of drugs of abuse, including cocaine and amphetamine derivatives (Ferrée, 2016).

Treatment of rodents with a P2X3R antagonist diminished opioid tolerance (Tai et al., 2010) and tolerance to morphineinduced antinociception ( $\mathrm{Ma}$ et al., 2015). Lead-induced neuroinflammation via P2X7R may be responsible for the intensification of morphine tolerance (Baranowska-Bosiacka et al., 2016). Opiate-induced changes in brain adenosine levels may be associated with opiate addiction and withdrawal ( $\mathrm{Wu}$ et al., 2013).

Adenosine, acting via $\mathrm{A}_{2 \mathrm{~A}} \mathrm{R}$, regulates addiction induced by cocaine. Women are more sensitive to cocaine and are therefore more vulnerable to becoming addicted. Adenosine antagonists may be an effective treatment (Broderick and Malave, 2014). $A_{2 A}-D_{2}$ receptor-receptor interactions in the dorsal striatum are differentially affected by cocaine, which contributes to compulsive drug seeking (Pintsuk et al., 2016). 
Interactions between striatal $\mathrm{A}_{2 \mathrm{~A}}$ and glutamate (mGlu5) receptors modulate the drug-seeking effects of methamphetamine (Wright S.R. et al., 2016). Methamphetamine produces alterations in adenosine receptor expression in the nucleus accumbens (Kavanagh et al., 2015). $\mathrm{A}_{2 \mathrm{~A}} \mathrm{R}$ antagonism in dorsomedial striatum reduces methamphetamine addiction (Furlong et al., 2015). Adenosine $\mathrm{A}_{2 \mathrm{~A}} \mathrm{R}$ integrate the rewarding and motivational behaviours of methamphetamine (Chesworth et al., 2016). Behavioural sensitisation provides a model in animals for drug craving that underlies human addiction and sensitisation to amphetamine was reduced by $\mathrm{P} 2 \mathrm{Y}_{1} \mathrm{R}$ antagonists in the mesocortico-limbic dopaminergic system (Krügel et al., 2013).

$\mathrm{A}_{2 \mathrm{~A}} \mathrm{R}$ agonist treatment can help counteract nicotine addiction (Jastrzebska et al., 2014). $\mathrm{A}_{2 \mathrm{~A}} \mathrm{R}$ are a potential target for the treatment of alcohol abuse (Micioni Di Bonaventura et al., 2012; Houchi et al., 2013). Regulation in adenosine signalling in striatal circuits in alcohol addiction was reviewed (Nam et al., 2013). $A_{1} R$ signalling contributes to the regulation of basolateral amygdala excitability and to the pathophysiology of alcohol addiction (Rau et al., 2014). A review of alcohol addiction discusses the role of adenosine (Michalak and Biala, 2016). P2X4R modulate synaptic signalling associated with alcohol addiction (Franklin et al., 2014; Khoja et al., 2016).

\section{Epilepsy}

Early focus was on the role of P1R in epileptic seizures, but P2X4R and P2YR, but especially P2X7R antagonists have been recently explored as neuroleptic agents (see Burnstock, 2015b; Engel et al., 2016; Rassendren and Audinat, 2016; Beamer et al., 2017; Cieslak et al., 2017). Seizure-induced increases in microglial process numbers were reduced and kainateinduced seizure behaviours were exacerbated in $\mathrm{P}_{2} \mathrm{Y}_{12} \mathrm{KO}$ mice (Eyo et al., 2014). Antiepileptic consequences of deep brain stimulation may be mediated by P1R activation (Miranda et al., 2014). P2X3R expression was upregulated in epileptic rats and humans and it was postulated that P2X3R antagonists might be therapeutically effective (Zhou X. et al., 2016). The release of adenosine and ATP during on-going epileptiform activity was measured with microelectrode biosensors (Frenguelli and Wall, 2016). ATP levels were increased during epileptic seizures, which were not of neuronal origin (Lietsche et al., 2016). There is an insightful Editorial about purinergic signalling-induced neuroinflammation in epilepsy (Engel, 2016).

A role for post-transcriptional control of the P2X7R expression has been proposed and therapeutic targeting of microRNA-22 was suggested to prevent development of epilepsy and inflammation (Jimenez-Mateos et al., 2015). Purinergic signalling, via P2X7R, regulates neonatal seizures associated with hypophosphatasia, an inherited metabolic bone disease characterised by spontaneous seizures (Sebastián-Serrano et al., 2016). P2X7R antagonists were effective against hypoxia-induced neonatal seizures in mice (Rodriguez-Alvarez et al., 2017).

\section{Migraine}

The role of ATP in migraine was initially suggested to involve vascular events (see Burnstock and Verkhratsky, 2012; Haanes and Edvinsson, 2014). Later, P2X3R in nociceptive brain areas, including the thalamus and trigeminal nucleus, were investigated and their interaction with $\mathrm{P}_{2} \mathrm{Y}_{1} \mathrm{R}$ in trigeminal neurons (Hullugundi et al., 2014; Marchenkova et al., 2016). The therapeutic potential of antagonists to P2X7R for migraine treatment has been proposed (Gölöncsér and Sperlágh, 2014), as well as P2X3R and P2X2/3R antagonists (Kilinc et al., 2015; Yegutkin et al., 2016). Reviews have been published about the roles and therapeutic potential of purinergic signalling in the aetiology of migraine (Cieslak et al., 2015; Yegutkin et al., 2016).

\section{Neuropathic Pain}

$\mathrm{P} 1 \mathrm{R}$ and $\mathrm{P} 2 \mathrm{R}$ involvement in neuropathic pain has been discussed in reviews (Burnstock and Sawynok, 2010; Burnstock et al., 2011; Burnstock, 2014a, 2016c). The discovery by Inoue and colleagues that antagonists to $\mathrm{P} 2 \mathrm{X} 4 \mathrm{R}$ on microglia are effective against neuropathic pain was particularly important (see Tsuda, 2016). Antagonists to $\mathrm{P} 2 \mathrm{X} 7 \mathrm{R}$ and $\mathrm{P} 2 \mathrm{Y}_{12} \mathrm{R}$ also act on microglia to reduce neuropathic pain (see Tsuda and Inoue, 2016; Tsuda, 2017). $A_{3} R$ agonists delay the development of neuropathic pain (Janes et al., 2016). Glial $\mathrm{P} 2 \mathrm{Y}_{2} \mathrm{R}$ are potential targets for the management of trigeminal-related pain (Magni et al., 2015). Pannexin 1 and P2X7R interactions have been suggested to play a role in chronic pain (Bravo et al., 2015). P2X7R antagonists have been recommended for the treatment of central post-stroke pain (Kuan et al., 2015). A review discusses the use of P2XR subtype antagonists for the treatment of central neuropathic pain (Kuan and Shyu, 2016). Purinergic signalling in the spinal dorsal horn and anterior cingulate cortex appear to be involved in neuropathic pain (Tsuda et al., 2017). P2X3 and P2X2/3R blockade has also been claimed to reduce chronic pain (Cantin et al., 2012; Xu et al., 2012; Giniatullin and Nistri, 2013).

\section{Brain Tumours}

Neuroblastoma, a rare childhood tumour, expresses P2X7R, which mediate proliferation. The P2X7R appears to be a regulator of neuroblastoma metabolic activity, angiogenesis and growth and may be a target for neuroblastoma treatment (Amoroso et al., 2015; Gomez-Villafuertes et al., 2015). The action of the antitumor agent, temozolomide, was increased by the antiproliferative actions of P2X7R agonists and antagonists to $\mathrm{A}_{3} \mathrm{R}$ and $\mathrm{P} 2 \mathrm{Y}_{1} \mathrm{R}$ on human glioblastoma (D'Alimonte et al., 2015). P2X4R may also be involved in human neuroblastoma (Gualix et al., 2015).

$\mathrm{P} 2 \mathrm{X} 7 \mathrm{R}$ were over-expressed in human malignant gliomas and P2X7R antagonists decreased tumour cell numbers (Fang et al., 2015; Morrone et al., 2016; McLarnon, 2017). Extracellular nucleotides control glioma growth via $\mathrm{P} 2 \mathrm{X} 7 \mathrm{R}$ and $\mathrm{P} 2 \mathrm{Y}_{6} \mathrm{R}$ activation (Braganhol et al., 2015). P2X7 and $\mathrm{A}_{2 \mathrm{~A}} \mathrm{R}$ activation leads to release of cytokines by macrophages, which was prevented by antagonists to these receptors (Bergamin et al., 2015). P2X7R antagonists blocked the cell cytotoxicity caused by irradiation for glioma (Gehring et al., 2015). Purine nucleoside phosphorylase is released from rat C6 glioma cells, contributing to the purinergic system homeostasis and exhibiting a pathophysiological role (Giuliani P. et al., 2017). 
It was claimed that $\mathrm{K}_{\mathrm{ATP}}$ channels are associated with tumorigenesis of human glioma ( $R u$ et al., 2014). A 3 R blockade enhances the actions of antitumour drugs used against human glioblastoma stem-like cells (Torres A. et al., 2016). $A_{1}$ and $A_{2 B} R$ also sensitise glioblastoma stem cells to chemotherapy (Daniele et al., 2014). $\mathrm{P} 2 \mathrm{Y}_{2} \mathrm{R}$ interactions with caveolin-1 represents a novel target for human astrocytoma cells (Martinez et al., 2016).

\section{Sleep Disorders}

$\mathrm{P}_{2} \mathrm{Y}_{11} \mathrm{R}$ appear to be associated with narcolepsy (Kornum et al., 2011). Reduced endothelial dilation to ATP in cerebral arteries occurred in a rat model of obstructive sleep apnoea (Crossland et al., 2013). Adenosine is a key player in the regulation and maintenance of sleep-wake dependent neural activity changes, where dysregulation can lead to sleep-wake disorders (Holst et al., 2016). The $A_{2 A} R$ antagonist, SCH58261, overcame the blood-brain barrier dysfunction as a result of sleep restriction (Hurtado-Alvarado et al., 2016).

\section{CARDIOVASCULAR DISEASES}

Reviews about this topic are available (Erlinge and Burnstock, 2008; Headrick et al., 2013; Burnstock and Ralevic, 2014; Burnstock, 2015a; Burnstock and Pelleg, 2015; Ralevic, 2015; Sousa and Diniz, 2017).

\section{Heart Diseases \\ Heart Failure}

In chronic heart failure adenosine accumulates, probably as a result of lowered adenosine deaminase (ADA) gene expression and raised CD73 activity. Adenosine therapy mediated by $\mathrm{A}_{1} \mathrm{R}$ and $A_{3} R$ is cardioprotective for chronic heart failure (Greene et al., 2016; Voors et al., 2017). $\mathrm{A}_{1} \mathrm{R}$ agonists attenuate cardiac hypertrophy and prevent heart failure in a mouse model (leftventricular pressure-overload) and in a rat model (neonatal cardiac myocyte) (Chuo et al., 2016). $\mathrm{A}_{2 \mathrm{~B}} \mathrm{R}$ agonists exert stronger cardioprotective effects against cardiac ischaemia/reperfusion injury compared to $\mathrm{A}_{2 \mathrm{~A}} \mathrm{R}$ activation in rats (Ke et al., 2015). CD73 and $\mathrm{A}_{2 \mathrm{~B}} \mathrm{R}$ agonists have been considered as therapeutic agents for myocardial ischaemia. Genetic deletion of CD39 results in increased myocardial ischaemia-reperfusion injury (Smith et al., 2016). Inflammatory responses initiated during ischemiamediated immune injury may be regulated by adenosine (Boros et al., 2016).

Early studies were concerned with the role of adenosine in ischaemic and reperfusion injuries. However, there is also interest in the role of ATP. Application of ATP, prior to or just after cardiac ischaemia is cardioprotective (Ren et al., 2016). ATP released from the ischaemic myocardium causes reflex responses mediated by cardiac sympathetic afferent nerves (Dong et al., 2016). P2YR are important therapeutic targets in myocardial protection during ischemia/reperfusion (Djerada et al., 2017). $\mathrm{P} 2 \mathrm{Y}_{6} \mathrm{R}$ could be a therapeutic target to regulate cardiac hypertrophy (Clouet et al., 2016). There is increased expression of $\mathrm{P}_{2} \mathrm{Y}_{2}$ and $\mathrm{P} 2 \mathrm{X} 1 \mathrm{R}$ in the hearts of rats with congestive heart failure.
P2X4R are needed for neuroprotection via ischemic preconditioning (Ozaki et al., 2016). P2X3R expression increased in dorsal root ganglion (DRG) and superior cervical ganglia neurons, resulting in exaggerated sympathoexcitatory reflexes. NONRATT021972 siRNA decreases the upregulation of P2X7R and improves cardiac function after myocardial ischemia (Tu et al., 2016). Mitochondrial $\mathrm{K}_{\mathrm{ATP}}$ channels provide protection against myocardial ischemia/reperfusion injury (Wang et al., 2015; Shimizu and Calvert, 2016). $\mathrm{K}_{\text {ATP }}$ channels maintain high energy phosphates and myocardial perfusion in heart failure (Jameel et al., 2016).

\section{Myocardial Infarction}

A clinical trial (Acute Myocardial Infarction Study of Adenosine) concluded that infusion for $3 \mathrm{~h}$ of adenosine resulted in a reduction of infarct size (Yetgin et al., 2015; Bulluck et al., 2016). Protection against myocardial infarction was mediated by $\mathrm{A}_{1} \mathrm{R}$ in the rabbit heart. The $\mathrm{A}_{3} \mathrm{R}$ agonist IB-MECA produced cardioprotection against myocardial infarction (Tian et al., 2015). Microglial P2X7R in the rat hypothalamic paraventricular nuclei regulate the sympathoexcitatory responses in acute myocardial infarction (Du D. et al., 2015). Loss of mouse $\mathrm{P}_{2} \mathrm{Y}_{4} \mathrm{R}$ protects against myocardial infarction (Horckmans et al., 2015). CD39 reduced infarct size following ischaemia-reperfusion injury (Smith et al., 2016). P2 $\mathrm{Y}_{12} \mathrm{R}$ antagonists have been recommended for long-term protection of patients, post-myocardial infarction (Alexopoulos et al., 2016). The $\mathrm{K}_{\mathrm{ATP}}$ channel opener, natakalim, improves ventricular remodelling of congestive heart failure after myocardial infarction (Jin, 2016).

\section{Atrial Fibrillation}

Adenosine reduces post-operative atrial fibrillation (AF). Up-regulation of $\mathrm{A}_{2 \mathrm{~A}} \mathrm{R}$ involves abnormal calcium handling in AF. Prevention of $A_{2 A} R$ activation in patients with $A F$ may sustain uniform beat-to-beat responses at higher beating frequencies (Molina et al., 2016). Adenosine-guided pulmonary vein isolation following a randomised clinical trial was recommended to treat paroxysmal AF (Macle et al., 2015), although this has been queried in a more recent clinical trial (Ghanbari et al., 2016). ATP-induced AF has also been investigated (Hasebe et al., 2016). The efficacy of the $\mathrm{P}_{2} \mathrm{Y}_{12}$ antagonists ticagrelor and prasugrel are not affected in AF (Ondrakova et al., 2016). The roles of adenosine and ATP in atrial arrhythmias and fibrillation have been discussed (Jared Bunch, 2015; Belhassen and Michowitz, 2016).

\section{Supraventricular Tachycardia}

Acute therapy by ATP for paroxysmal supraventricular tachycardia was used in the late 1940's. In patients with paroxysmal supraventricular tachycardia bolus injection of Adenocard (adenosine) is clinically prescribed to slow conduction time via the atrioventricular node, via $A_{1} R$ (Sachdeva and Gupta, 2013). Treatment of paroxysmal supraventricular tachycardia by adenosine and ATP is discussed (Lerman, 2015). To provoke vasovagal reaction in syncope patients ATP and adenosine are administered together with the head-up tilt table test (Fragakis et al., 2015). 


\section{Cardiomyopathy}

Cardiomyopathy can be an inherited disease, but can occur as a result of vitamin B deficiency, amyloidosis, alcoholism or viral infections. ATP synthase disruption contributes to diabetic cardiomyopathy (Ni et al., 2016). P2X7R involvement in dilated cardiomyopathy has been reported (Martinez et al., 2015).

\section{Cardiac Fibrosis and myocarditis}

$\mathrm{P}_{2} \mathrm{Y}_{11} \mathrm{R}$ agonists reduce cardiac fibrosis (Certal et al., 2015). Extracellular nucleotide regulation of signalling in cardiac fibrosis has been discussed (Novitskaya et al., 2016). The P2X7R antagonist, A740003, reduces experimental autoimmune myocarditis, suggesting a treatment for clinical myocarditis (Zempo et al., 2015).

\section{Angina}

ATP injections to treat angina pectoris associated with coronary disease were used during the 1940s, while AMP was used to treat angina. ATP treatment for patients with coronary insufficiency was also used early. Intracoronary administration of adenosine results in angina pain. Intracoronary administration of adenosine in patients with unstable angina produced decreased myonecrosis and improved coronary blood flow (Kizilirmak et al., 2015). In vascular pain, which encompasses pelvic and ischaemic pain, migraine and angina, it is thought that release of ATP from endothelial cells during reactive hyperaemia after vasospasm diffuses through the microvascular wall to activate P2X3R on perivascular sensory nerves to send impulses that travel to pain centres in the brain via the spinal cord (Joseph et al., 2015). P2X2/3R expressed on airway nociceptive sensory nerves mediate cardiovascular reflexes in conscious rats (Hooper et al., 2016).

\section{Cardiac Transplants}

Responses of the transplanted human heart to adenosine show supersensitivity. Protection of cardiac grafts from cold ischaemia/reperfusion injury is caused by donor pretreatment with AMP-activated protein kinase (Yang C. et al., 2016). Treatment with P2XR antagonists prolongs cardiac transplant survival.

\section{Vascular Diseases Hypertension}

It has been proposed in a recent review (Burnstock, 2017) that there are five different ways that purinergic signalling can contribute to the development of hypertension:

(1) ATP released as a cotransmitter from sympathetic nerves together with noradrenaline (NA) contributes, via P2X1R, to the vasoconstriction that results from increased sympathetic vasomotor activity in hypertension. Therefore, P2X1R antagonists should be useful for the treatment of hypertension, especially since there is a substantial increase in ATP relative to NA released from sympathetic nerves in spontaneously hypertensive rats (Brock and Van Helden, 1995; Goonetilleke et al., 2013).
(2) Release of ATP from endothelial cells by shear stress as a result of changes in blood flow acts on P2YR and $\mathrm{P} 2 \mathrm{X} 4 \mathrm{R}$ on endothelial cells to release nitric oxide (NO) resulting in vasodilation. Introduction of $\mathrm{P}_{2} \mathrm{Y}_{1}, \mathrm{P}_{2} \mathrm{Y}_{2}$, and P2X4 agonists would cause increased vasodilation in hypertension.

(3) Brain stem and hypothalamic neurons mediate sympathetic nerve activity. Recent studies show that P2X3R antagonists are antihypertensive, due to reduced sympathetic nerve activity as a result of increased peripheral P2X3R-mediated carotid body chemoreceptor reflexes (Pijacka et al., 2016).

(4) P2X7R in the kidney contribute to the pathophysiology of hypertension and $\mathrm{P} 2 \mathrm{X} 7 \mathrm{R}$ antagonists may have promise as clinical antihypertensive agents.

(5) In hypertensive patients adenosine activates the vascular renin-angiotensin system. P1R agonists have been suggested for the treatment of hypertension (Ho M.F. et al., 2016).

Recently, $\mathrm{P} 2 \mathrm{Y}_{6} \mathrm{R}$ were shown to age-dependently promote vascular remodelling in a mouse model, an effect inhibited by MRS2578, suggesting that $\mathrm{P}_{2} \mathrm{Y}_{6} \mathrm{R}$ are a therapeutic target for the prevention of age-related hypertension (Sunggip et al., 2017).

\section{Atherosclerosis}

ATP signalling influences the development of atherosclerosis (Burnstock, 2008a; Ferrari et al., 2015). Endothelial and smooth muscle cell proliferation are promoted by adenosine and ATP in atherosclerosis via $\mathrm{P} 2 \mathrm{Y}_{1}, \mathrm{P}_{2} \mathrm{Y}_{2}$, and $\mathrm{P} 1 \mathrm{R}$. In a human model, adenosine, via $\mathrm{A}_{2 \mathrm{~A}} \mathrm{R}$, modulates foam cell formation (Reiss and Cronstein, 2012). $A_{2 B}$ and $A_{3}$ antagonists reduce atherosclerotic plaque development. There are increased concentrations of circulating adenosine $5^{\prime}$-diphosphate (ADP) and ATP in atherosclerosis (Jalkanen et al., 2015). Uridine 5'-triphosphate (UTP), via $\mathrm{P} 2 \mathrm{Y}_{2} \mathrm{R}$, induces expression of vascular cell adhesion molecule-1 in coronary artery endothelial cells, which leads to the monocyte recruitment associated with atherosclerosis development. Upregulated $\mathrm{P}_{2} \mathrm{Y}_{2} \mathrm{R}$ mediate intimal hyperplasia in collared rabbit carotid artery. $\mathrm{P} 2 \mathrm{Y}_{1} \mathrm{R}$ antagonists are a therapeutic target for neointima formation (Liu R. et al., 2015). Endothelial $\mathrm{P} 2 \mathrm{X} 4 \mathrm{R}$ play a more important role in intense proliferation in atherosclerosis than $\mathrm{P} 2 \mathrm{Y}_{2} \mathrm{R}$. ATP, by inducing leukocyte recruitment in mice, contributes to atherogenesis, via $\mathrm{P}_{2} \mathrm{Y}_{2}, \mathrm{P}_{2} \mathrm{Y}_{6}, \mathrm{P} 2 \mathrm{X} 4$, and $\mathrm{P} 2 \mathrm{X} 7 \mathrm{R}$. P2X7R are over-expressed in atherosclerosis and P2X7R deficiency leads to less plaque formation (Stachon et al., 2016). CD39 mRNA-coated stents may be a treatment for atherosclerosis (Abraham et al., 2015).

Atherosclerosis of coronary vessels is called coronary artery disease or coronary artery syndrome. P2 $\mathrm{Y}_{12} \mathrm{R}$ antagonists combined with aspirin are beneficial for patients with acute coronary syndrome (De Luca et al., 2016; Rollini et al., 2016). $A_{2 A} R$ on coronary arteries have also been claimed to be involved in coronary artery disease (Gariboldi et al., 2017). 


\section{Vascular Injury, Angiogenesis and Restenosis}

An initiating event in the pathogenesis of vascular diseases is often vascular injury. Injured cells release ATP, which, together with adenosine, evoke endothelial and smooth muscle cell growth, proliferation, migration and death. Adenosine, following breakdown of ATP, is protective against ischaemic injury. $\mathrm{P} 2 \mathrm{Y}_{12} \mathrm{R}$ antagonists are prescribed as prevention against ischaemic stroke (Liu F. et al., 2015). P2 $\mathrm{Y}_{2} \mathrm{R}$ mediate regulation of endothelial inflammation and angiogenesis (Gidlöf et al., 2015).

Sustained control of proliferation of endothelial and smooth muscle cells as a result of P1 and P2YR activation during vascular remodelling in restenosis after angioplasty has been reported and therapeutic possibilities are being explored (Burnstock, 2002). Activation of $A_{2 B} R$ stimulates angiogenesis in human microvascular endothelial cells (Du X. et al., 2015). Adenosine, via $A_{2 A} R$, stimulates wound healing and angiogenesis following tissue injury in mice. CD39 administration decreases injuryinduced platelet deposition and recruitment of leukocytes and inhibits neointimal hyperplasia. A review discusses the vascular actions of P2XR in renal injury (Howarth et al., 2015).

\section{Thrombosis, Inflammation, and Stroke}

Nucleotides are extracellular mediators of vascular inflammation and thrombosis. Clopidogrel, a $\mathrm{P}_{2} \mathrm{Y}_{12}$ antagonist, inhibits aggregation in platelets and is widely prescribed for thrombosis and stroke (Sarafoff et al., 2012). Other P2Y 12 antagonists have been developed, including ticlopidine, cangrelor, ticagrelor, prasugrel, elinogrel, BX 667, and PSB 0739. P2Y ${ }_{1} \mathrm{R}$ antagonists also have antithrombotic actions and have been recommended as a complement to current $\mathrm{P}_{2} \mathrm{Y}_{12}$ anti-platelet strategies. The $\mathrm{P} 2 \mathrm{Y}_{6} \mathrm{R}$ may be a therapeutic target for systemic inflammatory responses. Review articles are available about purinergic signalling in thrombosis and inflammation, including the use of different oral or intravenous $\mathrm{P} 2 \mathrm{Y}_{12} \mathrm{R}$ antagonists (Tang et al., 2015; Nylander and Schulz, 2016; Rollini et al., 2017). P2X7R are pro-thrombotic and genetic $\mathrm{KO}$ of the gene for the P2X7R was shown to be protective in a mouse carotid artery thrombosis model (Furlan-Freguia et al., 2011). Adenosine, via $A_{2 A} R$ and $\mathrm{A}_{3} \mathrm{R}$, had antithrombotic effects (Cristalli et al., 1994; Hofer et al., 2013).

\section{Migraine}

Migraine pain involves two distinct cerebrovascular phases: an initial vasoconstriction (with no pain), followed by vasodilation (reactive hyperaemia) associated with pain. A purinergic hypothesis for migraine was proposed in 1981 (Burnstock, 1981). It was suggested that after the initial vasospasm ATP and adenosine (following breakdown of ATP) may mediate the vasodilation during reactive hyperaemia associated with pain. It was also hypothesised that stimulation of $\mathrm{P} 2 \mathrm{X} 3 \mathrm{R}$ on sensory nerve terminals located on the adventitia of the cerebral microvasculature by ATP contributed to the migraine pain. Data has also been presented recently that is consistent with the purinergic hypothesis of migraine pain (Yegutkin et al., 2016). P2X3R antagonists have been proposed as potential candidates for migraine treatment (Waeber and Moskowitz, 2003). The non-steroidal anti-inflammatory compound, naproxen, currently in use for migraine pain, blocks P2X3R-mediated responses in trigeminal neurons of the rat. Migraine could involve a chronic disorder of the sympathetic nervous system, where increased release of the sympathetic cotransmitter ATP contributes to the initial vasospasm.

Adenosine may also be involved in migraine. Adenosine infusion resulted in symptoms that were migraine-like and withdrawal of the P1R antagonists theophylline and caffeine also resulted in migraine-like symptoms. Clinical trials with the adenosine uptake inhibitor dipyridamole, that results in increased extracellular adenosine, were halted due to the increase of migraine attacks in all patients. Overactive P2YR on glial cells may contribute to pain transduction during migraine. Reviews have been published about the role of purinergic signalling in the aetiology of migraine and the potential of purinergic compounds (Cieslak et al., 2015; Jacobs and Dussor, 2016).

\section{Diabetic Vascular Disease}

There is pre-junctional $\mathrm{A}_{1} \mathrm{R}$-mediated sympathetic neurotransmission and ATP-mediated endothelial vasodilatation in mesenteric arteries of streptozotocin (STZ) diabetic rats (Burnstock and Novak, 2013). There is enhanced $\mathrm{A}_{2 \mathrm{~A}} \mathrm{R}$-mediated increase in coronary flow in type 1 diabetic mice (Labazi et al., 2016). UTP, ATP and adenosine evoked vasodilation is reduced in the circulation of skeletal muscle of type 2 diabetic patients. Erythrocyte release of ATP is diminished in type 2 diabetics, supporting the view that a defect in the physiology of erythrocytes may contribute to diabetic vascular disease. Erythrocytes are less deformable in type 2 diabetes leading to lowered levels of deformation-induced ATP release. Low erythrocyte ATP release may contribute to the prevention and treatment of diabetic peripheral vascular disease (Richards et al., 2015). P2X7R expression by monocytes might play a role in the pathological changes of type 2 diabetes mellitus (Wu et al., 2015).

\section{Aortic Valve Disease}

ATP inhibits mineralisation of the aortic valve seen in calcific aortic valve disease (Côté et al., 2012). Increased levels of ectonucleotidase are found in calcific aortic valve disease and inhibition of ectonucleotidase with ARL67156 prevented disease development in rats.

\section{Blood Cell Diseases}

Adenosine is a potential therapeutic target for the prevention and treatment of sickle cell disease (Fu and Davies, 2015). The circulating levels of adenosine are elevated in pregnant women with sickle cell disease (Ashimi et al., 2015). Amyloid- $\beta$ peptide inhibits ATP release from erythrocytes, suggesting that in AD, vascular amyloid peptide may play a role.

\section{DISEASES OF THE AIRWAYS}

Reviews covering the early literature are available (Burnstock et al., 2012a; McGovern and Mazzone, 2014).

Inflammation occurs in most diseases of the airways, including chronic obstructive pulmonary disease (COPD), 
dyspnea, asthma, cystic fibrosis (CF), allergy, infection and injury. Purine nucleotide release from airway epithelial cells is raised in inflammatory processes and has an important role in the pathophysiology of chronic lung disease. P2X7R are a therapeutic target in lung hypersensitivity reactions seen in chronic inflammatory responses. P2X7R modulate lung inflammatory, fibrotic and functional changes in silicosis, an occupational lung disease (Monção-Ribeiro et al., 2014). P2X4R mediate acute airway inflammation by regulating dendritic cell function (Wiesler et al., 2016). Vagal parasympathetic reflex bronchoconstriction has a role in inflammatory airway disease. Mucin hypersecretion is stimulated in various respiratory diseases and silencing of MUC8 by siRNA increased P2 $\mathrm{Y}_{2} \mathrm{R}$-induced airway inflammation (Cha et al., 2015). P2 $\mathrm{Y}_{2} \mathrm{R}$ have also been claimed to downregulate MUC5AC gene expression (Jeong et al., 2016). Nucleotides released during airway inflammation activate $\mathrm{P}_{2} \mathrm{Y}_{6} \mathrm{R}$ leading to further release of inflammatory cytokines (Hao et al., 2014). Anti-inflammatory effects of adenosine in the lung have been described involving immune cells.

\section{Asthma}

A role of adenosine in asthma has been considered for many years, because it is a powerful bronchoconstrictor in asthmatic, but not healthy lungs and P1R antagonists have been used for the treatment of asthma (see Cicala and Ialenti, 2013). It was suggested earlier that the bronchoconstriction evoked by adenosine in asthma was as a result of indirect actions by release of leukotrienes, histamine or endothelin (see Burnstock et al., 2012a). In early reports, $A_{2 A}, A_{2 B}$, and $A_{3} R$ were all claimed to mediate inhibition of allergic airway inflammation and mast cells were shown to be involved. Expression of adenosine receptors on monocytes from patients with asthma contributes to the progression of the disease (Yuryeva et al., 2015). $A_{2 A} R$ are involved in the immunological pathogenesis of asthma (Wang et al., 2016).

The roles of nucleotides in asthma has gained attention. In human lung mast cells, ATP is an important modulator of histamine release. Attenuated P2X7R function gives protection from asthma and was suggested to be age related. ATP in sputum was significantly elevated in patients with asthma, which was correlated with the percentage of neutrophils in sputum (Soma et al., 2016). Impaired P2X1R-mediated adhesion in eosinophils from asthmatic patients has been reported (Wright A. et al., 2016). P2 $\mathrm{Y}_{6} \mathrm{R}$ activation was effective for treatment of a mouse model of asthma (Chetty et al., 2016).

In allergic asthma, inhalation of allergens, such as pollen spores or house dust mite allergen, or triggers such as viral infection or air pollution, trigger inflammation. For an immune response to be triggered by allergens, activation of dendritic cells is required. In allergic asthmatics, bronchoconstriction following inhaled adenosine was mediated by $\mathrm{A}_{1} \mathrm{R}$. Selective antagonists to $\mathrm{A}_{2 \mathrm{~B}} \mathrm{R}$ improve inflammatory conditions in allergic asthma (Basu et al., 2017). ATP plays a role in inflammation in allergic asthma by recruitment and activation of inflammatory cells. $\mathrm{P}_{2} \mathrm{Y}_{1} \mathrm{R}$ are involved in allergic airway inflammation, probably by regulating maturation of dendritic cells. It has also been proposed that $\mathrm{P} 2 \mathrm{Y}_{2} \mathrm{R}$ in the respiratory epithelium are important sensors for airborne allergens. P2X7R have been implicated in the pathophysiology of allergy-induced lung inflammation. Activating P2X7R on hematopoietic cells, namely eosinophils or dendritic cells, may be a therapeutic approach to treat allergic asthma. Oxatomide, an anti-allergic antihistamine, is claimed to also act as a P2X7R antagonist (Yoshida et al., 2015). P2R act as modulators of rat eosinophil recruitment in allergic inflammation (Alberto et al., 2016). Asthmatic patients exhibit hypersensitivity to aerosolised ATP, but the effects of ATP are not mediated by adenosine (Basoglu et al., 2017).

\section{Chronic Obstructive Pulmonary Disease (COPD)}

COPD emphysema and chronic bronchitis. COPD is caused by gas or noxious particles, particularly in tobacco smoke, trigging a lung inflammatory response that when in the larger airways is called chronic bronchitis while in the alveoli is called emphysema.

The role of adenosine receptors in COPD has been the main emphasis to date. Combined stimulation of $\mathrm{A}_{2 \mathrm{~B}}$ and glucocorticoid receptors in epithelial cells of human airways induces genes that have anti-inflammatory potential for COPD (Greer et al., 2013). $A_{2 B} R$ on human mast cells were claimed to be a strategic target for COPD and inhaled $\mathrm{A}_{2 \mathrm{~A}} \mathrm{R}$ agonists have been used for the treatment of COPD. $A_{1}, A_{2 B}$, and $A_{3} R$ antagonists have also been used to treat COPD (Basu et al., 2017).

ATP is implicated in COPD as well as adenosine. COPD is characterised by up-regulation of ATP in bronchoalveolar lavage fluid, which promotes inflammation and tissue degradation. ATP-induced pulmonary vasodilation occurs in patients with COPD. Activation of P2X7R signalling by cigarette smoke appears to play a role in the pathogenesis of emphysema. Cigarette smoke induces neutrophil ATP release via P2X7 and probably $\mathrm{P}_{2} \mathrm{Y}_{2} \mathrm{R}$ activation. Aerosolised ATP exacerbates the symptoms of COPD (Basoglu et al., 2015). A valuable review about purinergic signalling and COPD has been published recently (Pelleg et al., 2016).

\section{Airway Infections}

Antibiotics, including erythromycin, are used widely for the treatment of lower and upper respiratory tract infections. Erythromycin blocks the P2XR-mediated $\mathrm{Ca}^{2+}$ influx and could represent one mechanism by which it exerts its effects. The airway epithelium has a role in activating the innate immune response during lung bacterial infections to fight the infection. Overexpression of the ectonucleotidase, CD39, promotes bacteriainduced inflammation, mediated largely by $\mathrm{P} 2 \mathrm{X} 7 \mathrm{R}$ in mouse airways. It has been proposed that P2X7R agonists together with low molecular weight anti-tuberculosis medicines could be used to treat multi-drug-resistant tuberculosis (TB) (Soares-Bezerra et al., 2015), although caution was advised as a polymorphism of the P2X7R was reported to increase the risk of recurrence of TB (Fernando et al., 2007). In infectious inflammatory diseases the roles of P2X7R and ectonucleotidases have been reviewed (Morandini et al., 2014). Polymorphisms of the P2X7R gene are 
associated with the prognosis and risk of human TB (Zheng X. et al., 2017). Data has been presented to support the view that P2X7R antagonists should be used to treat the aggressive forms of TB (Amaral et al., 2014). Selective, orally bioavailable and potent ATP synthase inhibitors show activity against both nonreplicating and replicating TB (Singh et al., 2015). P2X7R contain the spread of Toxoplasma gondii in vivo (Corrêa et al., 2017). The role of purinergic signalling in a mouse model of pneumococcal meningitis has been explored (Zierhut et al., 2017). The authors showed that although P2X7R activated the NLRP3 inflammasome/IL-1 $\beta$ pathway that mediates inflammation in pneumococcal meningitis, neither suramin nor brilliant blue $G$ affected the disease, possibly because of meningitis-associated down-regulation of brain P2X7R expression and/or a decrease in ATP levels in cerebrospinal fluid.

Adenosine protects against Streptococcus pneumoniae infection of the lungs by pulmonary neutrophil recruitment regulation (Bou Ghanem et al., 2015). Macrophages that engulf bacteria produce adenosine that suppresses sensitisation in response to early-life infections (Pei and Linden, 2016). Chemokine release and leukocyte recruitment are modulated by nucleotides in inflamed airways via an action on P2YR on immune and epithelial cells. Mucociliary clearance is the initial defence against infections of the airways. Airway epithelium releases ATP into the surface liquid layer that controls mucus clearance via P2R and, following breakdown to adenosine, also through P1R. Pulmonary TB patients had higher ADA activity in bronchoalveolar lavage fluid and in the sputum.

Infection with the malaria protozoan parasite, Plasmodium falciparum, induces ATP release from erythrocytes. Rupture of erythrocytes releases ATP during the blood-stage of $P$. chabaudi malaria that increases P2X7R expression on $\mathrm{CD} 4^{+} \mathrm{T}$ cells. Platelet ADA, CD39, and CD73 expression was reduced in Trypanosoma evansi infected rats. A review about purinergic signalling and malaria-infected erythrocytes is available (Huber, 2012). Haemolysis produced by leukotoxin, a bacterial virulence factor, was increased by ATP release and P2XR activation of human erythrocytes.

P2X7R activation regulates inflammatory responses during acute viral infection (Lee et al., 2012) and is involved in the exacerbated immune response seen during influenza virus infection (Leyva-Grado et al., 2017). ATP, released by activated macrophages and damaged cells, modulates lung inflammation in pneumonia in cattle. Both pulmonary microvascular endothelial cells and epithelial cells expressed P2X7R mRNA.

The pneumovirus respiratory syncytial virus commonly causes childhood lower respiratory tract diseases. It reduces alveolar clearance, probably via UTP, released by the bronchoalveolar epithelium following infection, suggesting that $\mathrm{P}_{2} \mathrm{Y}_{2} \mathrm{R}$ antagonists may be therapeutically important for the treatment of severe respiratory syncytial virus bronchiolitis (Vanderstocken et al., 2012). Rhinoviral stimuli and ATP signalling contribute to human bronchial smooth muscle production of IL-33 by severe asthmatics (Calvén et al., 2015). ATP is involved in the expression and release of a major airway mucin, MUC5AC, mainly via $\mathrm{P} 2 \mathrm{Y}_{2} \mathrm{R}$ and it was suggested that modulation of this pathway could be useful clinically for mucus hypersecretion following viral infections (Shishikura et al., 2016).

\section{Lung Injury}

Acute respiratory stress syndrome and lung injury can lead to respiratory failure. There is a protective effect of ATP- $\mathrm{MgCl}_{2}$ in ischaemia-reperfusion lung injury. Alveolar macrophages contribute substantially to chronic lung inflammation development, including silicosis, idiopathic pulmonary fibrosis, hypersensitivity pneumonitis, sarcoidosis, and asbestosis.

Alveolar macrophages express P2X7R, which stimulate the IL-1 to IL-5 proinflammatory cytokine cascade and may be clinically relevant in lung hypersensitivity reactions occurring due to chronic inflammation. P2X7R are involved in the pathophysiology of LPS-induced lung injury and LPS-induced inflammation occurs independently of $\mathrm{P}_{2} \mathrm{Y}_{1} \mathrm{R}$ (Liverani, 2017). There is up-regulation of pulmonary $\mathrm{P} 2 \mathrm{X} 4$ and $\mathrm{P} 2 \mathrm{X} 7 \mathrm{R}$ in both acute and chronic lung injury and P2X7R deletion, but not P2X4 deletion, was lung protective (Hafner et al., 2017). The initial inflammatory cells recruited during lung injury are pulmonary neutrophils and P2X7R antagonists reduced neutrophil infiltration and proinflammatory cytokine levels (Mishra et al., 2016).

Neuroendocrine body cells lining the lung epithelium at intervals, release ATP in response to distension, which then stimulates P2X3R to activate vagal sensory fibres that originate in the nodose ganglion (Brouns et al., 2003). This mechanism may control reflex responses to noxious gases and hyperventilation. Ventilator-induced lung injury may involve ATP release from neuroepithelial cell bodies in response to stretch and therefore may be therapeutically relevant.

Pulmonary fibrosis can be caused by injury. In patients with idiopathic pulmonary fibrosis $\mathrm{A}_{2 \mathrm{~B}} \mathrm{R}$ signalling may promote the production of inflammatory and fibrotic mediators. Extracellular adenosine levels are closely associated with the progression of pulmonary fibrosis (Luo F. et al., 2016). Adenosine production by CD73 enhanced radiation-induced lung fibrosis (Wirsdörfer et al., 2016). LPS caused increased expression of $A_{1}, A_{2 A}$, and $A_{2 B}$ and P2YR, and decreased expression of $A_{3} R$, while mechanical ventilation reduced $\mathrm{P}_{2} \mathrm{Y}_{4}$ mRNA levels. Both $\mathrm{A}_{2 \mathrm{~A}}$ and $\mathrm{A}_{2 \mathrm{~B}} \mathrm{R}$ were claimed to attenuate acute lung injury. Upregulated $A_{2 A} R$ activation is likely to improve the healing process after acute LPS-induced lung injury (Friebe et al., 2014). A protective role for $A_{2 B} R$ signalling has been reported to counter ischaemic lung injury (Densmore et al., 2017). Protective effects of $\mathrm{A}_{3} \mathrm{R}$ activation in attenuating reperfusion lung injury has also been reported. Inhibition of adenosine kinase attenuates acute lung injury (Köhler et al., 2016). Inhaled $\mathrm{A}_{2 \mathrm{~B}} \mathrm{R}$ agonists have been recommended for the treatment of acute lung injury (Hoegl et al., 2015). Adenosine is detrimental in lung recovery following hyperoxic lung injury (Davies et al., 2016). Reviews have been written about adenosine receptors as potential therapeutic targets for acute respiratory stress syndrome and acute lung injury (Schepp and Reutershan, 2008; Eckle et al., 2009). 


\section{Cystic Fibrosis (CF)}

Cystic fibrosis is hereditary as a result of a loss of function gene mutation in the cystic fibrosis transmembrane conductance regulator (CFTR) protein. Some outstanding reviews about purinergic signalling in CF have been published (Burnstock et al., 2012a; Della Latta et al., 2013). The regulation of ion transport by UTP and ATP in CF and normal human airway epithelium was proposed early, in retrospect probably via $\mathrm{P}_{2} \mathrm{Y}_{2}$ and/or $\mathrm{P}_{2} \mathrm{Y}_{4} \mathrm{R}$. P2R compounds for the treatment of CF to restore $\mathrm{Cl}^{-}$ secretion and/or inhibit $\mathrm{Na}^{+}$absorption are being investigated. Lipoxin $\mathrm{A}_{4}$, which stops inflammation, is inadequately produced in patients with CF, but it stimulates apical ATP release, which activates $\mathrm{P} 2 \mathrm{Y}_{11} \mathrm{R}$ resulting in epithelial repair (Higgins et al., 2014). With the R117H mutation associated with mild forms of $\mathrm{CF}$, there are faults with the gating conformational changes in the CFTR transmembrane domains, although the function of the nucleotide binding domains are unchanged (Yu et al., 2016). Gating of the R117H-CFTR was shown to be almost completely rectified by combined treatment with an ATP analogue $\left[N^{6}\right.$-(2-phenylethyl)-2'-deoxy-ATP], VX-770 (Ivacaftor, currently used to treat $\mathrm{CF}$ ) and nitrate ions $\left(\mathrm{NO}_{3}{ }^{-}\right)$. The authors concluded that future therapeutic developments might include the complementary use of ATP analogues with VX-770.

$\mathrm{Cl}^{-}$secretion across CF airway epithelia is restored by ATP by triggering calcium entry via P2XR. It was suggested that P2X4/6 heteromultimer receptors might be involved. CFTR activity is necessary for ATP release following erythrocyte deformation. CFTR is reduced or absent in CF and mechanical deformation of erythrocytes does not induce ATP release. Increasing nucleotide release via motion could have therapeutic implications for CF patients. Women with CF showed reduced survival compared with males. Oestrogen may lower breathing-induced release of ATP and ATP receptor-mediated $\left[\mathrm{Ca}^{2+}\right]_{\mathrm{i}}$ increase that induces $\mathrm{Cl}^{-}$secretion. Anti-oestrogens may therefore be beneficial in the treatment of $\mathrm{CF}$.

$\mathrm{A}_{1} \mathrm{R}$ agonists release $\left[\mathrm{Ca}^{2+}\right]_{\mathrm{i}}$ and activate $\mathrm{Cl}^{-}$and $\mathrm{K}^{+}$ currents in CF epithelial cells of the airways. $A_{1}$ and $A_{2 A} R$ participate in regulation of $\mathrm{Cl}^{-}$secretion in CF airway epithelial cells. Bronchoalveolar lavage from CF patients contained high concentrations of adenosine, correlating with higher $5^{\prime}$ nucleotidase and lower ADA activity. Adenosine regulates CFTR via $A_{2 B} R$. The exhaled breath condensate biomarker, adenosine, tracks changes in lung function in CF (Esther et al., 2013). There is increased airway adenosine metabolism in early CF (Esther et al., 2015).

\section{Lung Cancer}

Human lung A549 epithelial-like adenocarcinoma cells express $\mathrm{P} 2 \mathrm{UR}$ (i.e., $\mathrm{P} 2 \mathrm{Y}_{2}$ and/or $\mathrm{P}_{2} \mathrm{Y}_{4}$ ) that mediate increases in $\left[\mathrm{Ca}^{2+}\right]_{\mathrm{i}}$. In A549 cells there is calcium-dependent UTP and ATP release (with subsequent increase in adenosine levels). Erythromycin selectively inhibits influx of $\mathrm{Ca}^{2+}$ induced via P2X4R stimulation in A549 lung tumour cells. A549 cells express $\mathrm{P}_{2} \mathrm{Y}_{2}, \mathrm{P}_{2} \mathrm{Y}_{4}$, and $\mathrm{P} 2 \mathrm{Y}_{6}$, and $\mathrm{P} 2 \mathrm{X} 4 \mathrm{R}$. ATP induces dosedependent inhibition of growth of cell lines, including human mesothelioma (MER082), human papillary lung adenocarcinoma (H441), human squamous cell lung carcinoma (H520), human large cell lung carcinoma (H460), human small cell lung carcinoma (GLC4) and the PC14 lung adenocarcinoma cell line. ATP was released from Calu-3 cells derived from human lung adenocarcinoma, probably in response to $\mathrm{P}_{2} \mathrm{Y}_{2} \mathrm{R}$ activation. UTP, ATP and uridine diphosphate stimulate proliferation of lung tumour A549 cells through $\mathrm{P}_{2} \mathrm{Y}_{2}$ and $\mathrm{P}_{2} \mathrm{Y}_{6} \mathrm{R}$.

Autocrine ATP release and P2X7R activation affects the migration of human lung cancer cells (Takai et al., 2014). A significant increase in survival of non-small cell lung cancer patients with high P2X7R expression was identified compared to patients with low P2X7R expression (Boldrini et al., 2015). Nucleotides released by radiochemotherapy induce chemotaxis, adhesion and proliferation of human lung cancer cells and metastasis was inhibited in immunodeficient mice by purinergic receptor antagonists (Schneider et al., 2015). An association of $\mathrm{P} 2 \mathrm{X} 7, \mathrm{P} 2 \mathrm{X} 4$, and $\mathrm{P} 2 \mathrm{Y}_{1} \mathrm{R}$ with distant metastatic lung tumours was observed and increased degradation of ATP and ADP by CD39, which influence tumour growth and metastasisation (Hofman et al., 2015). ATP promotes cell survival by inducing a long lasting and sustained increase in $\left[\mathrm{Ca}^{2+}\right]_{\mathrm{i}}$ in lung cancer cells (Song et al., 2016). ATP binding cassette $\mathrm{E} 1$ promotes growth, invasion and metastasis of lung adenocarcinoma cells (Tian Y. et al., 2016). Ecto-5' -nucleotidase (CD73) inhibitors are currently under clinical trial to treat non-small cell lung cancer (Zhu et al., 2017).

$\mathrm{A}_{2 \mathrm{~B}} \mathrm{R}$ on host immune cells may participate in promoting angiogenesis and suppressing immunity and $\mathrm{A}_{2 \mathrm{~B}} \mathrm{R}$ KO mice showed reduced growth in a Lewis lung carcinoma isograft model. ATP increased the cytotoxicity of cisplatin, a common anti-cancer drug for the treatment of lung cancer, in the H460 carcinoma cell line. An $\mathrm{A}_{3} \mathrm{R}$ agonist inhibited cell proliferation by stopping the cell cycle and by apoptosis in A549 cells. Antagonism of $\mathrm{A}_{2 \mathrm{~A}} \mathrm{R}$ expressed by lung adenocarcinoma tumour cells inhibited their growth (MediavillaVarela et al., 2013). $A_{2 \mathrm{~A}} \mathrm{R}$ expression and CD73 have opposing prognostic effects in non-small cell lung cancer (Inoue et al., 2017).

Cachexia often occurs in lung cancer patients. In clinical trials, infusion of ATP in advanced non-small cell lung cancer patients contributed beneficially by increasing body weight, muscle strength and quality of life, as well as enhancing survival (Agteresch et al., 2003). ATP has been claimed to reduce radiation-induced damage in patients with non-small cell lung cancer (Swennen et al., 2008).

\section{Chronic Cough}

The therapeutic promise of P2X3R antagonists for the treatment of chronic cough was first recognised by Ford and Undem (2013). P2X3R are expressed by airway afferent nerves and mediate hypersensitivity of the cough reflex, which is dramatically reduced by the oral P2X3 antagonist, AF-219 (Abdulqawi et al., 2015). Central $A_{1} R$ were shown to suppress cough (El-Hashim et al., 2016). 


\section{Pleurisy}

Inosine contributed with adenosine to exert, via $\mathrm{A}_{2} \mathrm{R}$, antiinflammatory effects in pleural inflammation (da Rocha Lapa et al., 2012).

\section{Lung Allograft}

Ecto-5'-nucleotidase (CD73) reduced rejection of airway allografts by stimulating $\mathrm{A}_{2 \mathrm{~A}} \mathrm{R}$, which is a negative modulator of lymphocyte recruitment into the allograft. P2X7R antagonists prolong mouse lung allograft survival (Liu et al., 2014).

\section{DISEASES OF THE SPECIAL SENSES}

Purinergic signalling is involved in the physiology of the nasal organs, ear, eye and tongue. Purinergic drugs are being explored for corneal injury, retinal detachment, glaucoma, dry eye, retinitis, uveitis, rhinosinusitis, diabetic retinopathy, macular degeneration, noxious odour damage, Ménière’s disease, sensorineural deafness, tinnitus and taste defects (Burnstock, 2006; Housley et al., 2009).

\section{Eye}

The early literature up to 2006 was reviewed (Burnstock, 2006) and there are more recent reviews covering the treatment of ocular diseases by purinergic drugs (Guzman-Aranguez et al., 2014; Sanderson et al., 2014; Beckel et al., 2016; Lee et al., 2016; Reichenbach and Bringmann, 2016).

Glaucoma is characterised by progressive degeneration of retinal ganglion cells and visual loss. Elevated intraocular pressure reduction is the treatable risk factor for glaucoma. P2X7R antagonists are being explored for the treatment of glaucoma (Krizaj et al., 2014; Pérez de Lara et al., 2015; Sakamoto et al., 2015). There is elevation of extracellular ATP and upregulation of NTPDase1 in animal models of chronic glaucoma (Lu et al., 2015). Cromakalim, a $\mathrm{K}_{\mathrm{ATP}}$ channel opener, lowers intraocular pressure (Roy Chowdhury et al., 2015, 2016). Evidence for the use of adenosine receptor antagonists for the treatment of glaucoma has also been presented (Zhong et al., 2013; Agarwal and Agarwal, 2014). Ap 4 A improves adrenergic anti-glaucomatous therapeutic effectiveness (Loma et al., 2015) and has recently been claimed to be an effective compound for the treatment of glaucoma (Fonseca et al., 2017).

Treatment for dry eye by a long lasting $\mathrm{P} 2 \mathrm{Y}_{2} \mathrm{R}$ agonist, diquafosol, was developed by Inspire Pharmaceuticals, Inc. and is currently in use in Japan and Korea (see Lau et al., 2014).

A $\mathrm{P} 2 \mathrm{Y}_{2} \mathrm{R}$ agonist, INS37217, enhances subretinal fluid reabsorption and is recommended for the treatment of retinal detachment (Maminishkis et al., 2002; Meyer et al., 2002).

P2X7R antagonist and $A_{3} R$ agonists have been implicated for the treatment of diabetic neuropathy and retinopathy (Sugiyama, 2014; Reichenbach and Bringmann, 2016), photoreceptor neurodegeneration ( $\mathrm{Hu}$ et al., 2015; Ho T. et al., 2016), retinitis (Corso et al., 2016) and uveitis (Zhao R. et al., 2016).

The roles of $\mathrm{P}_{2} \mathrm{Y}_{2}$ and $\mathrm{P} 2 \mathrm{X} 7 \mathrm{R}$ in corneal wound healing have been reviewed (Minns and Trinkaus-Randall, 2016; Minns et al.,
2016). P2X7R activation in oxysterol cytotoxicity may be a target for the treatment of age-related macular degeneration (Olivier et al., 2016).

\section{Ear}

In the auditory system ATP depressed sound-evoked action potentials in the auditory nerve by stimulating P2YR. Acoustic over-stimulation can cause permanent hearing loss due to damage and death of cochlea hair cells. Noise exposure promotes the release of ATP into endolymph. ATP regulates hearing sensitivity and could be useful to treat sensorineural deafness, Ménières disease and tinnitus. UTP infusion into the deafened guinea pig inner ear rescued auditory neurons (Fransson et al., 2009). P2XR-mediated control of cochlear gap junctions may be protective by reducing hearing sensitivity to noise stress (Zhu and Zhao, 2012), perhaps via P2X2R (Yan et al., 2013; Mittal et al., 2016). Susceptibility to hearing loss and noise-induced neural injury in the mouse cochlea is regulated by $\mathrm{A}_{2 \mathrm{~A}} \mathrm{R}$ (Vlajkovic et al., 2017).

\section{Olfactory Organs}

Purinergic receptors are expressed in the nasal mucosa, including P2X3R on olfactory neurons. There is enhanced odour sensitivity in the presence of antagonists to P2R suggesting that endogenous ATP at a low level normally decreases odour responsiveness. Chemosensory trigeminal neurons express $\mathrm{P} 2 \mathrm{X} 2 \mathrm{R}$, which contribute to control of odour recognition (Housley et al., 2009). Activation of P2X3R negatively modulates the odour response, indicating a protective strategy for olfactory sensory neurons (Yu, 2015). Olfactory nerves and secretory cells in the vomeronasal organ express purinoceptors. Heatshock protein induction by noxious odour damage is inhibited by P2R antagonists in vivo. The inhibitory effect of ATP in odour responses may contribute to the reduction of odour sensitivity following exposure to noxious fumes and could be a new mechanism for neuroprotection (Yu and Zhang, 2014). ATP release following injury leads to post-injury neuroregeneration and may result in the development of therapies to restore loss of smell (Hayoz et al., 2012). P2 $\mathrm{Y}_{1} \mathrm{R}$ increase neuronal network activity in the developing olfactory bulb (Fischer et al., 2012). Purinergic signalling serves as a paracrine signal in regulating the neurogenesis of mouse olfactory epithelium (Gao L. et al., 2010). It has been claimed that purinoceptors are a therapeutic target to alleviate or restore loss of olfactory sensory neurons by the mycotoxin, satratoxin (Jia et al., 2011). Activation of $\mathrm{A}_{2} \mathrm{R}$ may be a novel therapeutic approach for enhancing nasal mucociliary clearance in chronic rhinosinusitis (Hua et al., 2013).

\section{Tongue}

ATP is a key neurotransmitter in the taste system, acting largely via P2X2/3 heteromultimer receptors (Kinnamon and Finger, 2013; Vandenbeuch et al., 2015). Consequently, disruption of taste function may be an unintentional consequence of therapeutic trials of pain, chronic cough and other conditions using purinergic P2X3R antagonists (Vandenbeuch et al., 2013). 


\section{IMMUNE SYSTEM AND INFLAMMATION}

$\mathrm{P} 2 \mathrm{X} 7, \mathrm{P}_{2} \mathrm{Y}_{1}$, and $\mathrm{P} 2 \mathrm{Y}_{2} \mathrm{R}$ on immune and inflammatory cells are important in immunomodulation and inflammation, and the purinergic contribution to neuroinflammation underlying neuropathology has been discussed in several recent reviews (Jacob et al., 2013; Takenouchi et al., 2014; Di Virgilio and Vuerich, 2015; Beamer et al., 2016). The role of P2X7R in particular in diseases related to neuroinflammation and the use of P2X7R centrally penetrant antagonists has been highlighted (Baudelet et al., 2015; Di Virgilio, 2015; Gentile et al., 2015; Burnstock, 2016b; Danquah et al., 2016; Karmakar et al., 2016; Rech et al., 2016; Corrêa et al., 2017; Giuliani A.L. et al., 2017; Jiang et al., 2017; Zheng B. et al., 2017). It has been claimed that activation of P2X1R on neutrophils and platelets is involved in regulation of thrombo-inflammation (Oury et al., 2015). $\mathrm{P} 2 \mathrm{Y}_{12} \mathrm{R}$ activation modulates sepsis-induced inflammation (Liverani et al., 2016). Adenosine, acting mainly via $A_{2 A} R$, is also involved in neoplastic and inflammatory and immune-mediated disease states (Antonioli et al., 2014a; da Rocha Lapa et al., 2014; Cekic and Linden, 2016; Ingwersen et al., 2016; Zhang X. et al., 2016; Faas et al., 2017).

\section{INFECTION}

Reviews have been published concerned with purinergic signalling in immune cell trafficking at sites of infection (Ferrari et al., 2016b) and P2X7R in infectious inflammatory diseases (Morandini et al., 2014). Apoptosis of macrophages via ATP-stimulated P2X7R leads to the killing of the mycobacteria they contain, which may lead to new strategies to combat bacterial infections. The cytotoxic effects of ATP on macrophages are through P2X7R, while the bactericidal actions of ATP (and UTP) may be though $\mathrm{P}_{2} \mathrm{Y}_{2} \mathrm{R}$. There is a valuable article about purinergic signalling in infection and autoimmune diseases (Savio and Coutinho-Silva, 2016). The P2X7R is a potential target for the treatment of Clostridium perfringens type $\mathrm{C}$ infection (Nagahama et al., 2015). P2X7R activation modulates cell death during Porphyromonas gingivalis infection (Almeidada-Silva et al., 2016). P2X7 and P2X4R activation is protective during severe Escherichia coli infection (Greve et al., 2017). P2X7R develop the inflammatory response associated with sepsis (Santana et al., 2015) and might serve as a therapeutic target to ameliorate brain damage in sepsis (Savio et al., 2016). $\mathrm{A}_{2 \mathrm{~A}} \mathrm{R}$ have also been recommended as a therapeutic target to treat sepsis (Sivak et al., 2016). The role of purinergic signalling in the immune response in sepsis has been reviewed (Ledderose et al., 2016). ATP protects against sepsis through P2X7R on macrophages by enhancing intracellular bacterial killing (Csóka et al., 2015). Chlamydiae are intracellular bacterial pathogens and these infections are influenced by inflammasomes and purinergic signalling (Pettengill et al., 2016). Purines modulate the inflammatory response in rats infected by Cryptococcus neoformans (de Azevedo et al., 2016). ADP facilitates monocyte recruitment in bacterial infection (Zhang X. et al., 2016). ATP synthase has been proposed as a target to kill Mycobacterium tuberculosis (Tantry et al., 2017). Purinergic enzymatic activities in lymphocytes and cardiomyocytes modulated the inflammatory responses of mice infected by T. cruzi (do Carmo et al., 2017). Adenosine restored LPS-inhibited chemotaxis via $A_{1} R$, making this a promising therapeutic strategy for infectious diseases (Xu et al., 2017).

Infection by Schistosoma mansoni, a parasitic blood fluke, results in thymic atrophy. The cloning and characterisation of a P2XR (schP2X) from S. mansoni was the first non-vertebrate ATP-gated ion channel, which could be an alternative drug target to treat schistosomiasis (see Burnstock and Kennedy, 2011). Purinergic signalling influences the immune response to infection by Leishmania, a protozoan parasite (Chaves et al., 2014; Figueiredo et al., 2016). Purinergic signalling is involved in Trichomonas vaginalis parasite infection (Menezes and Tasca, 2016). The dysfunction of P1, P2Y and P2X7R and NTPDases are likely to contribute to morbidity due to human schistosomiasis (Silva, 2016). P2X7R are important in parasite control as they regulate $\mathrm{T}$ effector cells and inflammation during L. amazonensis infection (Figliuolo et al., 2017a). A commentary about ATP as an initiator of immunity to parasitic infections has been published (Nelson et al., 2017).

P2X7R modulate the antiviral and inflammatory processes that occur during Dengue virus-2 infection (Corrêa et al., 2016) and exacerbate the immune response that occurs during influenza virus infection (Leyva-Grado et al., 2017). P2X4R antagonists reduce herpetic pain (Matsumura et al., 2016). Both P2X4 and $\mathrm{P} 2 \mathrm{X} 7 \mathrm{R}$ are involved in hepatitis $\mathrm{C}$ virus infection (Manzoor et al., 2016). Purinoceptors are putative targets for the treatment of HIV-1 infection (Pacheco et al., 2014). Ribavirin, an adenosine analogue, exhibited potent antiviral activities (Hao et al., 2017).

\section{ENDOCRINE DISEASES}

Purinoceptors are expressed widely by endocrine glands (Burnstock and Knight, 2004). Actions of purines have been described in the pituitary gland, with implications for pathological as well as physiological states. $5^{\prime}$-Nucleotidase activity in platelets is changed by hyper- and hypothyroidism and could be a further mechanism by which alterations in thyroid hormones are related to vascular diseases. The role of purinergic signalling in thyroid hormone activities in health and disease has been reviewed (Silveira et al., 2013). In the ovary, follicular atresia involves cellular degeneration that is due to apoptosis evoked by P2X7R activation in both somatic and germinal follicular cells. Adiponectin, secreted by adipocytes, is anti-inflammatory, protecting against fatty liver disorder, insulin-resistant type 2 diabetes and atherosclerosis. A review including the limited knowledge of purinergic signalling in the endocrine system has been published (Burnstock, 2014b).

\section{Diabetes}

Fibroblast P2YR are impaired in type 2 diabetes, which leads to lower glucose uptake, indicating that P2YR could be therapeutically useful as antidiabetic drugs. Analogues of the $\mathrm{P} 2 \mathrm{Y}_{1} \mathrm{R}$ agonist, 2-methylthio ADP, have been developed for 
the treatment of type 2 diabetes (Yelovitch et al., 2012). P2YR activation potentiates insulin secretion making it a promising therapeutic target for type 2 diabetes (Zhang et al., 2015b; DeOliveira et al., 2017). Adenosine, acting via $A_{2 A} R$, increases pancreatic $\beta$-cell proliferation, and has been suggested as a therapeutic target for diabetes (Schulz et al., 2016). Uridine adenosine tetraphosphate may be a therapeutic target for diabetes (Matsumoto et al., 2015).

There is an enhancement of P2X7R-induced apoptosis on the retinal microvasculature in early diabetes. P2X7R located on glucagon-containing $\alpha$ cells in pancreatic islets in STZ-induced diabetic animals increase and they migrate centrally to replace missing insulin-containing $\beta$ cells. P2X7R antagonists have been proposed as a therapeutic target to cause immunosuppression and tolerance induction in pancreatic islet transplantation (Aikin, 2013; Vergani et al., 2013). NONRATT021972 siRNA decreases the expression of $\mathrm{P} 2 \mathrm{X} 7 \mathrm{mRNA}$ and protein in DRG, reducing mechanical and thermal hyperalgesia in type 2 diabetic rats (Liu et al., 2016c). Type 1 diabetes impairs P2X7R signalling in osteocytes that affects osteoblast function and maintenance of bone health (Seref-Ferlengez et al., 2016). STZ-induced type 1 diabetes was prevented in P2X7R KO mice (Vieira et al., 2016). Diabetic sympathetic neuropathy in type 2 diabetic rats was reduced by decreasing the expression of $\mathrm{P} 2 \mathrm{X} 7 \mathrm{R}$ in superior cervical ganglia (Wu et al., 2016). P2X7R are expressed in pancreatic cancer cells. P2X7R antagonists are likely to be effective therapeutic agents (Giannuzzo et al., 2016).

P2X3R antagonists have been proposed for the treatment of diabetic neuropathic pain (Guo et al., 2015; Zhang et al., 2015b; Peng et al., 2017; Rao et al., 2017). Recently P2X7R have also been claimed to be involved in painful diabetic neuropathy in rats (Liu et al., 2017). P2X7R polymorphisms are associated with severe diabetic neuropathic pain scores (Ursu et al., 2014). $\mathrm{A}_{1} \mathrm{R}$ agonists improve mechanical allodynia in a painful diabetic neuropathy mouse model (Katz et al., 2015).

There is up-regulation of hippocampal $\mathrm{A}_{2 \mathrm{~A}} \mathrm{R}$ in STZ-diabetes and $\mathrm{A}_{2 \mathrm{~A}} \mathrm{R}$ antagonists gave neuroprotection. $\mathrm{A}_{2 \mathrm{~A}} \mathrm{R}$ are a therapeutic target for diabetic retinopathy (Ibrahim et al., 2011). Reduced expression of $A_{1} R$ in pancreatic $\alpha$-cells could result in the development of type 1 diabetes (Yip et al., 2013). There is increased expression of $\mathrm{A}_{2 \mathrm{~B}} \mathrm{R}$ in women with gestational diabetes mellitus (Wojcik et al., 2014). In diabetic rats, $A_{2 B} R$ agonists improve erectile function (Wen et al., 2015). It was suggested that reduction in $\mathrm{A}_{3} \mathrm{R}$ expression/function may slow the progression of diabetic neuropathy (Yan H. et al., 2016). Blockade of ATP synthase interaction with cyclophilin $\mathrm{D}$ has been proposed as a promising therapeutic target to treat diabetic encephalopathy (Yan S. et al., 2016).

$\mathrm{K}_{\mathrm{ATP}}$ channels have neuroprotective effects in patients with type 2 diabetes (Liu R. et al., 2016; Rubaiy, 2016). P2Y 1 and $\mathrm{P} 2 \mathrm{Y}_{2} \mathrm{R}$ mediate relaxation of the rat corpus cavernosum and may improve erectile function in men with diabetes (Gür et al., 2009).

An $\mathrm{A}_{2 \mathrm{~A}} \mathrm{R}$ agonist was shown to enhance healing of chronic diabetic foot ulcers in a clinical trial (Squadrito et al., 2014; Montesinos et al., 2015). Adenosine kinase inhibitors attenuate inflammation in diabetic retinopathy (Elsherbiny et al., 2013a). Retinal inflammation in diabetic retinopathy is mediated by
ADA2 and the anti-inflammatory activity of $\mathrm{A}_{2 \mathrm{~A}} \mathrm{R}$ signalling is impaired with increased ADA2 activity (Elsherbiny et al., 2013b).

Several reviews about purinergic signalling in diabetes and its therapeutic potential are available (Burnstock and Novak, 2013; Cieslak and Roszek, 2014; Antonioli et al., 2015; Fotino et al., 2015; Kishore et al., 2015; Merighi et al., 2015; Vindeirinho et al., 2016). The involvement of purinergic signalling in diabetic nephropathy is discussed later in the Section on Kidney.

\section{OBESITY}

ATP released as a cotransmitter from sympathetic nerves, stimulates brown adipocytes. ATP stimulates lipogenesis in rat adipocytes, regulating fat stores not via established hormones. Adipocytes express two different P2YR subtypes and $\mathrm{P} 2 \mathrm{Y}_{11} \mathrm{R}$ activation inhibited insulin-stimulated lepton production and lipolysis stimulation. $\mathrm{P}_{2} \mathrm{Y}_{4} \mathrm{R}$ mediate inhibition of cardiac fat formation (Lemaire et al., 2016). The antiobesity effects of sesamol, a potent anti-inflammatory compound, is mediated by AMP-activated protein kinase (Go et al., 2017). Blocking $\mathrm{P}_{2} \mathrm{Y}_{6} \mathrm{R}$ activation in the CNS with the antagonist MRS2578 inhibits feeding in mice (Steculorum et al., 2017).

Some ATP effects are as a result of adenosine, after breakdown of ATP, which is also involved in the activities of adipocytes. Adenosine increased lipolysis and induced thermogenesis in brown adipocytes via $\mathrm{A}_{2 \mathrm{~A}} \mathrm{R}$ ( $\mathrm{Gnad}$ et al., 2014). They showed that $\mathrm{A}_{2 \mathrm{~A}} \mathrm{R}$ antagonists counteract high fat-induced obesity in mice. Administration of $\mathrm{A}_{2 \mathrm{~A}} \mathrm{R}$ agonists to obese mice caused improvements in glucose homeostasis and adipose tissue inflammation, suggesting that this may show promise for therapeutic treatment of obesity (DeOliveira et al., 2017).

There is disturbance of adiponectin secretion in obese patients and adiponectin release is controlled by ATP (Yamauchi and Kadowaki, 2013). A combination of $\mathrm{Ca}^{2+}$ and ATP augments vesicular release of adiponectin (Komai et al., 2014). Both ATP and adenosine have roles in the regulation of leptin secretion from adipocytes (Szkudelski, 2007). There is abnormal fat distribution in P2X7R KO mice (Beaucage et al., 2014). Manipulation of P2XR signalling may represent a novel therapeutic target in metabolically unhealthy obesity under inflammatory conditions (Pandolfi et al., 2016). There is protection of rats on high fat diet by adenosine (Lee, 2015). Perivascular adipose tissue of heavy smokers has increased expression of P2X7R and inflammasome components leading to increased release of inflammatory cytokines (Rossi et al., 2014).

\section{GUT DISORDERS}

Purinergic signalling plays an important role in a variety of gut activities (Liñán-Rico et al., 2015; Burnstock, 2016d; Chaudhury et al., 2016). ATP is a cotransmitter in non-adrenergic, non-cholinergic inhibitory nerves involved in peristalsis, a synaptic transmitter in submucosal and myenteric ganglia, it mediates mucosal secretion and vascular control of the 
gastrointestinal tract. Both interstitial cells of Cajal and enteric glial cells express P2R.

Investigations of purinoceptors as therapeutic candidates for gut disorders are underway (Antonioli et al., 2013; Ochoa-Cortes et al., 2014; Burnstock, 2016d).

\section{Inflammatory Bowel Disease (IBD)}

Nucleotides and their receptors are involved in the pathogenesis of IBD, of which ulcerative colitis (UC) and Crohn's disease are the main types. $\mathrm{P}_{2} \mathrm{Y}_{6} \mathrm{R}$ are highly expressed on the $\mathrm{T}$ cells infiltrating $\mathrm{IBD}$, suggesting that $\mathrm{P}_{2} \mathrm{Y}_{6} \mathrm{R}$ may play a role in the pathogenesis of IBD. P2X7R agonists are involved in colonic motor dysfunction associated with bowel inflammation in rats (Antonioli et al., 2014b) and are over-expressed in gut mucosa of patients with IBD. P2X7R KO mice are protected against gut inflammation (Figliuolo et al., 2017b). ATP activates mast cells, which further promote the inflammatory process (Kurashima and Kiyono, 2016). Oestrogen receptor $\beta$ activation plays a therapeutic role in IBD by down-regulation of $\mathrm{P} 2 \mathrm{X} 3$ and $\mathrm{P} 2 \mathrm{X} 7 \mathrm{R}$ (Ma B. et al., 2016). The roles of P2X7R in IBD are discussed in reviews (Kurashima et al., 2015; Diezmos et al., 2016).

P2X7R-expressing enteric neurons are differentially affected in UC based on their chemical codes (da Silva et al., 2015). In a later paper, it was shown that UC affected secretory and vasodilatory neurons, enteric sensory neurons and enteric glia of the submucosal plexus expressing P2X7R (da Silva et al., 2017). The P2X7R antagonist, A438079, down-regulated the production of proinflammatory cytokines and attenuated murine colitis, indicating that P2X7R mediate inflammatory responses during UC (Wan P. et al., 2016). Activation of P2X7R triggers the death of mucosal regulatory T cells (Figliuolo et al., 2017b). There was P2XR enhancement in an animal model of UC. MicroRNA-16 and microRNA-206 have pro-inflammatory roles in UC by downregulating $A_{2 A}$ and $A_{3} R$ expression (Tian T. et al., 2016; Wu et al., 2017).

There is increased expression of P2X7R in the inflamed mucosa in Crohn's disease, suggesting that P2X7R antagonists may be targets for treatment of Crohn's disease (Neves et al., 2014; Eser et al., 2015; Wan P. et al., 2016; Figliuolo et al., 2017b). P2X3R antagonists are being explored as therapeutic agents against colic and UC pain (Eser et al., 2015), as well as antibodies against P2X3R (Deiteren et al., 2015; Diezmos et al., 2016; Shcherbatko et al., 2016).

The potential of P2X3R to treat irritable bowel syndrome has been suggested. In diarrhoea-predominant irritable bowel syndrome there is increased expression of $\mathrm{P} 2 \mathrm{Y}_{1}$ and $\mathrm{P} 2 \mathrm{Y}_{2} \mathrm{R}$, which was associated with abdominal pain (Luo Y. et al., 2016). P2X7R in DRG play a role in transmission of the nociceptive signal from the gut (Liu S. et al., 2015). A review about purinergic mediators in the control of intestinal inflammation and irritable bowel syndrome is available (Kurashima et al., 2015).

\section{Motility Disorders}

Purinergic signalling is involved in gastrointestinal motility disorders, including diarrhoea and constipation (Jiménez et al., 2014). Purinergic fast inhibitory junction potentials are impaired in Hirschsprung's disease (Jiménez et al., 2015). P2X4 and P2X7R activity was potentiated in enteric glia isolated from mice treated long-term with morphine (Bhave et al., 2017).

A review focuses on the pathophysiological roles of P2YR in inflammation (Wan H.X. et al., 2016). Aged mice have a lower ability to deal with inflammation evoked by Candida albicans infection, due to a lower gut density of $A_{2 A} R$, which reduce inflammation (Rodrigues et al., 2016).

\section{Colorectal Cancer}

Apoptosis is induced by extracellular ATP and ATP also reduced growth of primary cultures of colorectal carcinomas, possibly via $\mathrm{P} 2 \mathrm{Y}_{2} \mathrm{R}$ (see Burnstock and Di Virgilio, 2013; Wan H.X. et al., 2016). Purinergic responses of HT-29 cells, a colonic adenocarcinoma cell line, are mediated by $\mathrm{P} 2 \mathrm{Y}_{2}$ and/or $\mathrm{P} 2 \mathrm{Y}_{4} \mathrm{R}$. Enhanced expression of $\mathrm{A}_{2 \mathrm{~B}} \mathrm{R}$ on colorectal cancer cells that are proliferating suggests that antagonists to $\mathrm{A}_{2 \mathrm{~B}} \mathrm{R}$ may be a promising therapeutic target for colorectal cancer (Ma et al., 2010; Molck et al., 2016). 8-Chloro adenosine inhibited growth of colorectal cancer cell lines 80514 and HCT116 in vivo and in vitro. A phase $\mathrm{II}$, multi-centre study of $\mathrm{CF} 101$, an $\mathrm{A}_{3} \mathrm{R}$ agonist, showed stabilisation of tumour in $35 \%$ of refractory metastatic colorectal cancer patients. P2X7R antagonists reduce tumour occurrence in a colitis-associated cancer mouse model (Hofman et al., 2015). There is high expression of the ectonucleotidase CD39 in human rectal adenocarcinoma (Zhang et al., 2015a).

\section{DISEASES OF THE KIDNEY}

Reviews are available (Burnstock et al., 2014a; Menzies et al., 2017; Oyarzun et al., 2017), including one that focuses on the roles played by ATP released as a cotransmitter from sympathetic nerves in renal diseases (Burnstock and Loesch, 2017).

Purinoceptors are richly expressed in the glomerulus, renal vascular system and nephron in the kidney, including subtypes involved in the regulation of glomerular filtration, renin secretion and transport of nutrients, ions, water and toxins. The distribution of NTPDases 1 and 2 parallels the distribution of P2R in the kidney, influencing physiological and pathophysiological renal events (see Burnstock et al., 2014a). Adenosine and ATP have protective effects against renal ischaemic-reperfusion injury, and have been investigated for transplantation-induced erythrocytosis and chronic renal failure treatment. It has been suggested that nephron luminal P2R are part of an epithelial 'secretory' defence mechanism against harmful particles or bacteria. Increased expression of P2X7R in glomerulonephritis, hypertension, polycystic kidney disease (PKD) and diabetes may be therapeutically relevant as a novel treatment of kidney failure.

\section{Renal Injury and Failure}

ATP contributes to kidney remodelling and progression toward chronic renal failure (with associated sympathetic overactivity). P2X7R are expressed weakly in healthy glomerulus, but after glomerular injury (e.g., in hypertension and diabetes) are upregulated, mostly in podocytes, but also in mesangial and 
endothelial cells (Vonend et al., 2004). P2X7R play a role in altered intracellular calcium homeostasis in peripheral blood mononuclear cells of chronic kidney disease patients (Lajdova et al., 2012). P2X7R mediate deleterious renal epithelial-fibroblast cross-talk. P2X7R antagonists may be therapeutic targets to prevent and treat morbidity and mortality in kidney injury (Zarjou and Agarwal, 2011). P2X7R antagonists were protective against ischaemic acute kidney injury in mice (Yan et al., 2015). Arterial calcification is a feature in chronic kidney disease patients and ATP signalling is involved (see Fish et al., 2013). Cyclosporine is a potent immunosuppressive agent, but with limitations because of its side effect of nephrotoxicity. However, treatment with ATP following pre-treatment with verapamil greatly reduces nephrotoxicity. ATP is an important contributor to innate immunity regulation in primary idiopathic nephrotic syndrome. There is increased renal fibrosis in P2X4R-deficient mice following unilateral ureteric obstruction (Kim et al., 2014).

Adenosine mediates haemodynamic changes in adult renal failure. $\mathrm{A}_{2 \mathrm{~B}} \mathrm{R}$-mediated IL-6 induction contributes to renal fibrogenesis and this receptor has therapeutic potential for treatment of chronic kidney disease (Grenz et al., 2012). Dendritic cells and macrophages activated by $\mathrm{A}_{2 \mathrm{~A}} \mathrm{R}$ agonists attenuate acute renal injury (Li et al., 2012; Truong et al., 2016). There is a review describing $\mathrm{A}_{2 \mathrm{~A}} \mathrm{R}$ in acute kidney injury (Vincent and Okusa, 2015).

\section{Polycystic Kidney Disease (PKD)}

The genetic disorder PKD results in abnormal proliferation of tubular cells of the adult nephron, leading to progressive dilation of tubules and formation of fluid-filled cysts that destroy by compression neighbouring tissue. In the cysts ATP is released at high concentrations. The Caenorhabditis elegans nematode is an animal model for investigating basic molecular mechanisms underlying human autosomal dominant PKD; the C. elegans PKD-2 and LOV-1 proteins are homologues of human polycystin (PC)-1 and PC-2 proteins. Nucleotide release is involved in both fluid flow and pressure responses and its role in altered mechanosensory transduction in PKD is considered in a review (Patel and Honoré, 2010).

Cysts in the collecting ducts of the $c p k / c p k$ mouse model of congenital PKD express P2X7R, where they mediate cyst development. $\mathrm{P} 2 \mathrm{Y}_{1}, \mathrm{P} 2 \mathrm{Y}_{2}, \mathrm{P}_{2} \mathrm{Y}_{4}, \mathrm{P} 2 \mathrm{Y}_{6}, \mathrm{P} 2 \mathrm{X} 5$, and $\mathrm{P} 2 \mathrm{X} 7 \mathrm{R}$ were found on the lining epithelial cells of renal cysts in the rat Han:SPRD cy/+ model of autosomal dominant PKD. Expression of mRNA and protein for $\mathrm{P} 2 \mathrm{Y}_{2}, \mathrm{P} 2 \mathrm{Y}_{6}$, and $\mathrm{P} 2 \mathrm{X} 7 \mathrm{R}$ increased substantially as the disease developed. ATP inhibits renal cyst growth, via P2X7R. The P2X7R antagonists, oxidised ATP and A-438079, reduced cyst formation via extracellular signalregulated kinase-dependent pathways in a zebrafish model of PKD (Chang et al., 2011). Nucleotides in the lumen fluid of cysts stimulate P2YR resulting in increased growth of Madin Darby canine kidney-derived cysts. Knocking out PKD-1 gene expression increased $\mathrm{A}_{3} \mathrm{R}$ in human renal cells (Aguiari et al., 2009). There is a review about the functional and therapeutic importance of purinergic signalling in PKD (Ilatovskaya et al., 2016).

\section{Ischaemia}

Proximal tubular ATP declines rapidly in ischaemic acute renal failure. There was early recognition that there was restoration after ischaemia by perfusion of $\mathrm{ATP}-\mathrm{MgCl}_{2}$ and $\mathrm{A}_{3} \mathrm{R} \mathrm{KO}$ mice were shown to be protected against ischaemic renal failure. The ectonucleotidase CD73 protects the kidney from ischaemiareperfusion injury via production of adenosine and free radical reduction (Jian et al., 2012). The role of adenosine in protection from renal ischaemia-reperfusion injury has been investigated and discussed in reviews (Yap and Lee, 2012; Roberts et al., 2013; Sashindranath et al., 2017).

\section{Nephritis}

Glomerulonephritis is one of the leading causes of endstage renal disease. Increased expression of pro-apoptotic P2X7R has been demonstrated in both experimental and human glomerulonephritis, which suggests that P2X7R antagonists may have therapeutic potential (Deplano et al., 2013). It was shown later that P2X7R-deficiency attenuated renal injury in experimental glomerulonephritis (Taylor et al., 2009). P2X7R blockade attenuates lupus nephritis by inhibiting inflammasome activation (Zhao et al., 2013) and protects against cisplatin-induced nephrotoxicity (Zhang et al., 2014). The P2R antagonist, pyridoxal-phosphate-6azophenyl-2' $4^{\prime}$-disulfonate (PPADS), effectively inhibits mesangial cell proliferation in a rat mesangial proliferative glomerulonephritis model. Tubuloglomerular feedback is greatly reduced in Thy-1 nephritic rats, but exogenous $5^{\prime}$-nucleotidase improved it. In $\mathrm{P}_{2} \mathrm{Y}_{1} \mathrm{R} \mathrm{KO}$ mice, there is protection against fibrosis and death by renal failure as a result of experimental crescentic glomerulonephritis. Adenosine uptake inhibitors attenuated glomerulonephritis in mice. $\mathrm{A}_{2 \mathrm{~A}} \mathrm{R}$ agonists may treat macrophage-mediated experimental glomerulonephritis. $\mathrm{A}_{2 \mathrm{~A}} \mathrm{R}$ agonists reduced inflammation in mouse kidneys, suggesting a therapeutic approach for human lupus nephritis.

\section{Hypertension}

Hypertension is a feature of chronic renal disease; this is due largely to sympathetic overactivity as a result of afferent signals from the kidney and triggering sympathetic tone resetting by activation of hypothalamic centres (Orth et al., 2001). ATP is a cotransmitter released from sympathetic nerves, whose activity is increased in hypertension.

There is an enhanced P2R-mediated vasoconstriction of both efferent and afferent arterioles in chronic angiotensin IIinduced hypertensive rats, with predominant P2X1 and P2X7R control of glomerular haemodynamics (Franco et al., 2017). In connexin $30 \mathrm{KO}$ mice, epithelial sodium channels that are unable to respond to changes in sodium levels as a result of reduced paracrine ATP feedback regulation may play a role in salt-sensitive hypertension. Mice lacking $\mathrm{P} 2 \mathrm{Y}_{2} \mathrm{R}$ have salt-resistant hypertension. The $\mathrm{P} 2 \mathrm{X} 7 \mathrm{R}$ is expressed weakly in healthy kidney glomerulus, but expression is significantly increased in hypertension (Vonend et al., 2004; Menzies et al., 2015). P2X7R antagonists inhibited the development 
of renal injury and salt-sensitive hypertension in Dahl saltsensitive rats (Ji et al., 2012). Activation of $A_{1} R$ in mouse proximal tubules, modulated deoxycorticosterone acetate-salt hypertension.

\section{Diabetic Nephropathy}

Adenosine receptor agonists protect STZ-diabetic rats from nephropathy (Taskiran et al., 2016). A review discusses the role of $\mathrm{A}_{2 \mathrm{~B}} \mathrm{R}$ antagonists as therapeutic treatment of diabetic nephropathy (Quezada et al., 2013). CD73 generation of adenosine attenuates diabetic nephropathy (Tak et al., 2014). $\mathrm{A}_{3} \mathrm{R}$ antagonists reduced fibrosis in diabetic nephropathy (Kretschmar et al., 2016). AMP-activated protein kinase has been proposed as a potential treatment of diabetic nephropathy (Cameron et al., 2016). Chronic $\mathrm{A}_{2 \mathrm{~A}} \mathrm{R}$ stimulation prevents proteinuria and glomerular damage in experimental diabetes (Persson et al., 2015).

P2X7R agonists evoke renal inflammation and injury induced by high-fat diet in type 2 diabetes, suggesting that P2X7R antagonists might be of therapeutic interest (Solini et al., 2013). P2X7R expression was increased in the STZ diabetic rat model (Vonend et al., 2004). It has been suggested that P2X7R antagonists may be a useful coadjuvant treatment to delay the progression of diabetic nephropathy (Rodrigues et al., 2014). Data has been presented to suggest that activation of P2X7R contributes to the high prevalence of kidney disease found in diabetics (Rodrigues et al., 2014; Menzies et al., 2017). Deletion of the $\mathrm{P} 2 \mathrm{Y}_{2} \mathrm{R}$ reduced the development of nephrogenic diabetes insipidus polyuria induced by lithium. Blockade of $\mathrm{P} 2 \mathrm{Y}_{12} \mathrm{R}$ in the renal collecting duct alleviates nephrogenic diabetes insipidus (Zhang et al., 2015c).

\section{Nephrotoxicant Injury}

Drug-induced nephrotoxicity was reduced by ATP- $\mathrm{MgCl}_{2} \cdot \mathrm{A}_{1} \mathrm{R}$ antagonists are protective against cisplatin-induced acute kidney injury in rats (Gill et al., 2009). Adenosine antagonists are protective against acute renal failure. $A_{1} R$ antagonists also reduce nephrotoxicity induced by the immunosuppressive agent cyclosporine. Cyclosporine increases plasma adenosine levels in kidney transplant patients. $\mathrm{A}_{3} \mathrm{R}$ antagonism is effective against acute tacrolimus toxicity.

\section{Cancer}

P2X7R are strongly expressed on human embryonic kidney tumours, associated with increased proliferation (Adinolfi et al., 2012). The anthraquinone, emodin, inhibits human embryonic kidney cancer cell invasiveness by antagonising P2X7R (Jelassi et al., 2013). A prognostic indicator for post-operative cancerspecific survival of clear-cell renal cell carcinoma patients is P2X7R expression (Liu Z. et al., 2015).

\section{DISEASES OF THE LOWER URINARY TRACT}

The early literature has been thoroughly reviewed (Burnstock, 2014d; Keay et al., 2014).

\section{Urinary Bladder}

In contrast to laboratory animals, where the purinergic component of bladder parasympathetic cotransmission is about $50 \%$, in healthy human bladder it is approximately $2 \%$ although ATP receptors are expressed by the smooth muscle. In pathological conditions, however, such as neurogenic bladder, outflow obstruction and interstitial cystitis (IC), the purinergic component can be as much as $40 \%$ and is consequently a therapeutic target. Botulinum neurotoxin type A (BTXA) is often used to treat bladder incontinence, where it inhibits ATP release as well as acetylcholine (ACh) release both from parasympathetic nerves and urothelial cells. Bladder epithelial cell release of ATP from patients with IC was substantially higher compared to healthy controls. Dysfunction in micturition involves $\mathrm{P} 2 \mathrm{X} 3 \mathrm{R}$ in rats with chronic spinal cord-injury, suggesting that $\mathrm{P} 2 \mathrm{X} 3 \mathrm{R}$ antagonists could be used to treat neurogenic bladder dysfunction.

\section{Overactive Bladder (OAB) Syndrome}

There are valuable reviews that discuss the roles of purinergic signalling in OAB (Meng et al., 2012; Sacco et al., 2015). $\mathrm{OAB}$ syndrome, which increases in old age, is characterised by frequency, nocturia and urgency, with or without urge incontinence. Women with an OAB exhibit higher ATP levels in their urine compared to controls, suggesting that this could be used as a prognostic marker for detrusor overactivity (Cheng et al., 2013; Silva-Ramos et al., 2013). The increased urine ATP concentration is due to increased release of ATP from proliferating urothelium and reduced metabolism of ATP (SilvaRamos et al., 2013). P2X3R are present in the human bladder and, when upregulated, may play a role in the pathophysiology of $\mathrm{OAB}$.

Patients with metabolic syndrome, where the risk of developing cardiovascular disease and diabetes is increased, exhibit a higher incidence of OAB. P2X3R expression is increased in subepithelial sensory nerves resulting in increased bladder activity. $\mathrm{OAB}$ is common among $\mathrm{PD}$ patients and inhibition of overactivity by $\mathrm{A}_{2 \mathrm{~A}} \mathrm{R}$ antagonists occurs, probably by acting in the CNS to regulate the micturition reflex (Kitta et al., 2012). Uridine diphosphate via $\mathrm{P}_{2} \mathrm{Y}_{6} \mathrm{R}$ regulates abnormal smooth muscle activity in the $\mathrm{OAB}$ and increases contractions due to P2X1R activation (Yu W. et al., 2013). $\mathrm{P}_{2} \mathrm{Y}_{6}$-deficiency increases micturition frequency and reduces contractility in the mouse urinary bladder (Kira et al., 2017).

\section{Unstable Bladder}

Unstable bladder (also referred to as detrusor instability or detrusor hyperreflexia) is either neurogenic or idiopathic in origin. Neurogenic detrusor instability can occur as a result of a stroke, or pelvic or spinal cord injury, as well as in PD and MS. Neurogenic bladders are hyper-responsive to ATP, via P2X3R.

Expression of $\mathrm{P} 2 \mathrm{X} 2 \mathrm{R}$ in both urothelium and detrusor muscle of suprasacral spinal cord injury patients are similar to P2X2R expression in bladder tissue from idiopathic overactive bladder patients. ATP levels increased in the rat spinal cord-injured bladder that activates $\mathrm{P} 2 \mathrm{X} 3$ and $\mathrm{P} 2 \mathrm{X} 2 / 3 \mathrm{R}$ on afferent nerve fibres resulting in neurogenic bladder overactivity (Munoz et al., 2012). 
P2YR agonists ADP, UTP and uridine diphosphate originating in the urothelium augment the contractile overactivity following spinal cord injury. Sympathetic nerve hyperactivity may play a role in reflex sympathetic dystrophy where increased ATP would be released as a cotransmitter to activate P2X1R on smooth muscle resulting in increased contractions of the bladder and P2X3R on sensory nerve fibre terminals resulting in bladder reflexes and nociception. Inhibition of P2X7R expressed at spinal cord injury sites improved the dysfunction of neurogenic bladder (Munoz et al., 2017).

Urothelial cell ATP release (both resting and evoked) was substantially more from rat bladders following chronic spinal cord injury, contributing to bladder hyperactivity development. In a mouse bladder overactivity model, bradykinin facilitated the release of ATP from nerve terminals via prejunctional receptors (Fabiyi and Brading, 2006).

In the absence of $\mathrm{P} 2 \mathrm{X} 3 \mathrm{R}$ in $\mathrm{KO}$ mice, there is bladder hyperactivity (Cockayne et al., 2000; Vlaskovska et al., 2001). AF-219, a P2X3 and P2X2/3R antagonist that is metabolically stable and orally bioavailable, is being investigated as a treatment for urinary tract dysfunction (Ford and Cockayne, 2011).

Idiopathic detrusor instability patients show abnormal bladder purinergic transmission, which may contribute to the symptoms of OAB (Andersson and Hedlund, 2002; O'Reilly et al., 2002). There is greater potency of ATP via P2X1R for generating detrusor contractions in unstable bladder patients. Urothelial release of ATP from human bladders from patients with detrusor overactivity occurs in both idiopathic and neurogenic conditions (Kumar et al., 2010). Oxybutynin, administered chronically, results in a shift from muscarinic to purinergic transmission in the rat bladder wall. Expression of P2X3R is increased on suburothelial sensory nerve fibres in idiopathic detrusor overactivity patients (Liu H. et al., 2013).

\section{Interstitial Cystitis (IC)/Bladder Pain Syndrome}

The possible roles of ATP and adenosine in IC/bladder pain syndrome are discussed in a review (Veselá et al., 2012a). Hypoosmotic mechanical stimulation of urothelial cells released ATP that was substantially increased in cyclophosphamideinduced inflamed bladder (Smith et al., 2005). In rats with cystitis induced by hydrochloric acid, purinergic receptors were lost, although release of ATP from mucosal cells was increased.

Contractions of the rat bladder mediated by parasympathetic nerve stimulation and release of ATP and ACh, were reduced in cyclophosphamide-induced cystitis (Veselá et al., 2012b), while P2XR function in sensory neurons was enhanced. The purinergic component was abolished in the neurogenic bladder following desensitisation with $\alpha, \beta$-methylene ATP $(\alpha, \beta$-meATP). ATP release due to stretch from bladder urothelium from IC patients was greater compared to healthy urothelium and P2X3R expression was upregulated. Urothelial cells from cats with feline IC responded to $\alpha, \beta$-meATP, indicating increased expression of urothelial P2XR. There is mechanical hypersensitivity of the bladder in feline IC together with greater release of ATP from the urothelium; P2X1R expression is reduced and $\mathrm{P}_{2} \mathrm{Y}_{2} \mathrm{R}$ expression is lost.
$A_{1} R$ blockade during the initial phase of IC/bladder pain syndrome was suggested as a treatment for this condition (Aronsson et al., 2012). The $\mathrm{A}_{1} \mathrm{R}$ in rat urinary bladder is decreased in cyclophosphamide-induced cystitis (Veselá et al., 2011).

In neutrophils and macrophages in the submucosa of the bladder from mice with cyclophosphamide-induced haemorrhagic cystitis, expression of P2X7R is increased (Martins et al., 2012). Treatment with A-438079, a P2X7R antagonist, or genetic ablation of this receptor reduced nociceptive behaviour.

\section{Outflow Obstruction}

P2X1R expression in smooth muscle of the bladder was increased in the human obstructed bladder. In animal models of outlet obstruction, in vivo release of ATP from urothelial cells was increased compared to controls (Akino et al., 2011). Contractions to ATP were reduced in obstructed urinary bladder of rats (Sjuve Scott et al., 2004). There is P2X3R up-regulation in interstitial cells of Cajal in an experimental rat model of partial bladder outflow obstruction (Li et al., 2013). In the pig, partial bladder outlet obstruction resulted in reduced contractions in response to electrical field stimulation as well as to purinergic agonists (Milicic et al., 2006), but in the rat partial outlet obstruction model, contractions to ATP were increased after 2 weeks and 3 months (Murakami et al., 2008). Patients with bladder outflow obstruction release higher amounts of ATP into the urine (SilvaRamos et al., 2016).

\section{Botulinum Toxin A (BTXA) and ATP Release}

BTXA is increasingly being used to treat detrusor overactivity. In an early paper, it was shown that BTXA inhibited ATP as well as ACh release from parasympathetic nerves. Recently, BTX has been shown to inhibit ATP release from the urothelium (Chancellor et al., 2008; Ikeda Y. et al., 2012; Cruz, 2013). Daytime frequency, urgency, nocturia and pain were decreased by injection of BTXA into the human bladder. BTX is used to treat $\mathrm{OAB}$ syndrome and bladder hypersensory states (Apostolidis et al., 2005; Atiemo et al., 2005).

\section{Chronic Alcohol Consumption and Bladder Function}

Chronic consumption of ethanol impairs purinoceptor-mediated relaxation of detrusor smooth muscle of the rat (Calvert et al., 2002). Ethanol also alters neuronal P2XR.

\section{Diabetic Bladder}

Damage to human urinary bladder autonomic nerves and disturbances in micturition in diabetes have been recognised for many years. Urothelial release of ATP has been reported to play a role in bladder dysfunction in type 2 diabetes (Wang Z. et al., 2013). There is a transient increase in sensitivity of the STZ rat whole bladder preparation to $\alpha, \beta$-meATP. The cholinergic component of nerve-mediated contractions was reduced in diabetic rat and rabbit bladders, but the purinergic component was increased (Mumtaz et al., 2006).

In STZ mice, the urinary bladder had weaker nervemediated contractions in response to electrical field stimulation in contrast to normal mouse bladder. $\mathrm{Ca}^{2+}$ regulation of ATP release may be impaired in diabetes. Increased adenosine- 
and ATP-mediated relaxant responses, together with increased ATP-mediated contractile responses were observed in bladders from 8-week STZ diabetic rats. The enhanced contractions of STZ-diabetic rat bladders to nerve stimulation and ATP peaked between 6 and 9 weeks, but dropped to control levels by 12-20 weeks (Daneshgari et al., 2006). There is also an increase in release of ATP in STZ female rat bladders (Munoz et al., 2008). There is upregulation of P2X1R in the bladder of STZ-induced diabetes in the early phase, but downregulation of the P2X2R (Liu et al., 2008). Bladder overactivity occurring 2 months after induction of diabetes with STZ was accompanied by enhanced expression of $\mathrm{P} 2 \mathrm{Y}_{2}$ and $\mathrm{P} 2 \mathrm{Y}_{4} \mathrm{R}$ (Suadicani et al., 2009).

\section{Bacterial Infection}

Bacterial infection of the bladder causes urgency, urinary incontinence and overactivity. Urine ATP concentrations were reduced during episodes of bacteriuria (Walsh et al., 2013). An ATP assay method has been employed for many years to test for bacteriuria in urine.

\section{Bladder Cancer}

The growth of bladder cancer cells is reduced by ATP, both in vivo and in vitro (Shabbir et al., 2008; Shabbir and Burnstock, 2009). Doxorubicin, used to treat bladder cancer, has side effects, such as increased urgency and urinary frequency. Quercetin, a plant-derived flavonoid, prevents bladder cancer in cells lines, by suppressing cell proliferation and inducing arrest of the cell cycle or cell death by inhibiting the activity of ecto-nucleotidases (Rockenbach et al., 2013). In humans with bladder papillary carcinoma urothelial differentiation was correlated with the expression and localisation of P2X3 and P2X5R (Sterle et al., 2014). High CD73 immunoreactivity was associated with reduced bladder tumour progression (Wettstein et al., 2015). P2X7R protein favoured survival of patients with bladder urothelial cell carcinoma (Hu J. et al., 2016).

\section{Bladder Pain}

In 1995, P2X3 homomultimer and P2X2/3 heteromultimer receptors were cloned and were localised on sensory nerve endings. Purinergic mechanosensory transduction was proposed (Burnstock, 1999), suggesting that release of ATP from urothelial cells as a result of distension stimulates $\mathrm{P} 2 \mathrm{X} 3$ and $\mathrm{P} 2 \mathrm{X} 2 / 3 \mathrm{R}$ on suburothelial sensory nerve endings to activate high threshold nociceptive nerve fibres that reach the cortex pain centres (Burnstock, 2009). Distension of the bladder stimulates discharge in the nociceptive sensory nerves, which can be mimicked by ATP and can be inhibited by P2X3R antagonists (Vlaskovska et al., 2001). Stimulation of bladder P2X3R sensitises bladder afferent nerves, mimicking the sensitising effect of cyclophosphamideinduced cystitis. P2X3R are a potential target for therapeutic treatment of bladder pain. $\mathrm{P} 2 \mathrm{Y}_{2} \mathrm{R}$ are also expressed on bladder sensory nerves and are claimed to mediate increased stimulation of P2XR-mediated activity, playing a role in bladder pain syndrome (IC) (Chen et al., 2010).

\section{Urethra}

The principal non-adrenergic, non-cholinergic inhibitory transmitter to the urethra is NO, but a small component of purinergic neurotransmission is also involved (Andersson, 2001). ATP causes urethral relaxation, probably via $\mathrm{P} 2 \mathrm{Y}_{1} \mathrm{R}$, in pigs, guinea-pigs, rabbits and hamsters. The responses to non-adrenergic, non-cholinergic nerve stimulation were blocked by $\alpha, \beta$-meATP, indicating that the P2X1R subtype was involved.

Sensory nerve fibres supplying the urethra may release ATP during axon reflex activity. Prostaglandins, produced following stimulation of P2YR, contribute to contractions of the urethra in pathophysiological conditions. In the rat, SC19220 (a prostaglandin E1 antagonist), lowered detrusor tone leading to increased bladder capacity and decreased voiding efficiency. Some sensory functions in the urethra are mediated by afferent fibres that express P2X3R (Canda et al., 2006). A review that discusses the therapeutic opportunities offered by $\mathrm{K}_{\mathrm{ATP}}$ channels in the urethra is available (Kyle, 2014).

\section{Ureter}

The ureter motor innervation is sparse, perhaps because peristaltic activity is not neurogenic, but myogenic. The dominant nerves in the ureter are sensory, confined mainly to the suburothelial plexus. These nerves contribute to vesicoureteral reflux that activates reflexes that modulate urine delivery to the bladder.

ATP constricts the pig ureter, while intravesical adenosine evoked relaxation via $A_{2 B} R$. In the ureter, ATP, $\alpha, \beta$-meATP and adenosine evoked transient decreases in the frequency of peristalsis. In the rat ureter expression of P2X1R was shown on smooth muscle, while P2X5 and perhaps P2X7R are expressed on urothelium and P2X6R in the layer beneath the urothelium. $\mathrm{P} 2 \mathrm{X} 3 \mathrm{R}$ are localised on subepithelial sensory nerves that mediate nociception produced by release of ATP following distension of urothelial cells (Knight et al., 2002; Rong and Burnstock, 2004). Some ureter purinoceptors are likely to participate in long-term (trophic) events during development and regeneration, such as cell proliferation, differentiation, migration and cell death. The human ureter releases ATP in response to distension and human ureteric suburothelial sensory nerves express P2X3R (Calvert et al., 2008b). In a review of the pharmacology and physiology of the human ureter, it was proposed that purinergic receptors may prove to be analgesic targets to treat ureteral colicky pain and to facilitate ureteral stone passage (Canda et al., 2007).

Seven days after unilateral ureteral obstruction of wild-type mice, it was shown that there was increased expression of P2X7R associated with fibrogenic responses and inflammation in the cortex. However, in P2X7R KO mice, the alterations seen in the wild-type mice were not present. It was suggested that $\mathrm{P} 2 \mathrm{X} 7 \mathrm{R}$ antagonists may play a role in preventing renal interstitial fibrosis. In $\mathrm{A}_{2 \mathrm{~A}} \mathrm{R} \mathrm{KO}$ mice with unilateral ureteral obstruction, there was a substantially increased progression of renal interstitial fibrosis (Xiao et al., 2013).

\section{DISEASES OF THE LIVER}

In the liver, the two epithelial cell types that secrete bile, i.e., hepatocytes and cholangiocytes, express purinoceptor in the plasma membrane. Modulation of the release of ATP 
and purinergic signalling may be novel strategies to manage cholestasis and other bile flow disorders. Both quiescent and activated hepatic stellate cells (HSC) express purinergic receptors: $\mathrm{P}_{2} \mathrm{Y}_{2}$ and $\mathrm{P}_{2} \mathrm{Y}_{4} \mathrm{R}$ on quiescent and $\mathrm{P}_{2} \mathrm{Y}_{6} \mathrm{R}$ on activated HSC. P2YR on satellite cells could be a therapeutic target to treat or prevent liver fibrosis. Reviews concerned with purinergic signalling in liver disease are available (Burnstock et al., 2014b; Vaughn et al., 2014).

\section{Liver Injury, Inflammation, Immune Regulation and Repair}

Purinergic signalling regulates the immune response in the liver. In $\mathrm{A}_{1} \mathrm{R} \mathrm{KO}$ mice $\alpha$-naphthylisothiocyanate-induced cholestatic liver injury was decreased. CD39-deficient mice treated with adenosine, probably via $A_{2 A} R$, were protected from reperfusion injury (Sun et al., 2011). $A_{1} R$ antagonists abolished ischaemic preconditioning. After the resection of bile-duct-ligated cirrhotic livers, an $\mathrm{A}_{2 \mathrm{~A}} \mathrm{R}$ agonist improved liver function (Iskandarov et al., 2016). Inhalation of high concentrations of hydrogen protects against ischaemia/reperfusion injury through $\mathrm{A}_{2 \mathrm{~A}} \mathrm{R}$ activation (Li H. et al., 2017). Adenosine, via $\mathrm{A}_{2 \mathrm{~B}}$ or $\mathrm{A}_{3} \mathrm{R}$, accelerates the cell cycle during partial hepatectomy-induced liver regeneration in rats (Mendieta-Condado et al., 2007). In mice, ATL-146e, a selective $\mathrm{A}_{2 \mathrm{~A}} \mathrm{R}$ agonist, prevented concanavalin $\mathrm{A}$-induced acute liver injury.

$\mathrm{P} 2 \mathrm{Y}_{2} \mathrm{R}$ activation in mice makes a major contribution to endotoxin-induced acute liver injury (Samuel et al., 2010). In mice with acute liver injury, $\mathrm{P}_{2} \mathrm{Y}_{2} \mathrm{R}$ mediate neutrophil infiltration, regulating immune responses associated with death of hepatocytes. $\mathrm{P}_{2} \mathrm{Y}_{2} \mathrm{R}$ antagonists might be useful to treat inflammatory liver disease (Ayata et al., 2012). A review discusses the role of purinergic signalling in sterile liver injury (Oliveira et al., 2013).

Infusion of ATP- $\mathrm{MgCl}_{2}$ was shown early to improve hepatic function and survival following hepatic ischaemia and after reperfusion. During ischaemia there is a substantial loss of ATP from hepatocytes. Resistance of hepatocytes to hypoxia is promoted by $\mathrm{P}_{2} \mathrm{Y}_{2} \mathrm{R}$. In mice, CD39 deletion from natural killer (NK) cells reduced hepatic ischaemia/reperfusion injury, indicating that during liver regeneration ATP modulates NK cell function. UTP acting via $\mathrm{P}_{2} \mathrm{Y}_{2}$ and/or $\mathrm{P}_{2} \mathrm{Y}_{4} \mathrm{R}$ before induction of ischaemia attenuates post-ischaemic hepatocyte apoptosis resulting in a reduction of liver damage (Ben-Ari et al., 2009). Platelet aggregation triggered by ADP may have a role in ischaemia reperfusion injury (Schulte am Esch et al., 2010).

ATP released from sympathetic nerves and from hepatocytes, may participate in the regulation of liver repair. In $\mathrm{P}_{2} \mathrm{Y}_{2} \mathrm{R}$ $\mathrm{KO}$ mice, hepatocellular proliferation is impaired, indicating that ATP has a trophic role in liver regeneration and growth after injury (Thevananther et al., 2008). ATP release following partial hepatectomy in rats regulates liver regeneration. Apyrase (CD39/NTPDase1) reduces extracellular ATP allowing NK cells to play a role in the regulation of the immune response and to improve liver regeneration (Graubardt et al., 2013). After partial hepatectomy, liver regeneration is enhanced by the $\mathrm{K}_{\text {ATP }}$ opener, diazoxide (Nakagawa et al., 2012).
Hepatocyte lipoapoptosis contributes to hepatic inflammation in lipotoxic liver injury. Pannexin1 may contribute to hepatic inflammation by increasing ATP release in lipotoxic liver injury (Xiao et al., 2012). Acetaminophen (APAP), used to reduce pain and fever, may damage hepatocytes. In mouse APAP-induced inflammation models, full injury following an overdose involved P2X7R activation (Amaral et al., 2013). P2X7R activate hepatic caspase-1 as well as the migration of neutrophils into the liver, suggesting that ATP may play a major role in the development of inflammasomes following overdose of APAP (Hoque et al., 2012). A438079, a P2X7R antagonist, is protective against APAPinduced liver injury (Xie et al., 2013). P2X4R are involved in liver regeneration after partial hepatectomy in mice (Besnard et al., 2016). P2X1R-regulated IL-22 secretion is required for liver regeneration (Kudira et al., 2016).

\section{Fibrosis and Hepatic Stellate Cells (HSC)}

Liver fibrosis followed by cirrhosis is a common cause of liver failure. HSC are the main fibrogenic cells of the liver, which express nucleotide receptors that are functional (Kruglov et al., 2007) and mediate phospholipase D activity (Benitez-Rajal et al., 2006).

Adenosine $\mathrm{A}_{2 \mathrm{~B}} \mathrm{R}$ play pro-fibrotic roles in human $\mathrm{HSC}$ (Zhong et al., 2007). During fibrosis, HSC proliferate and undergo senescence and $\mathrm{A}_{2 \mathrm{~A}} \mathrm{R}$ mediate both these key processes, suggesting that $\mathrm{A}_{2 \mathrm{~A}} \mathrm{R}$ antagonists are potential antifibrotics (Ahsan, 2011). $A_{2 A} R$ contribute to the pathogenesis of hepatic fibrosis and $\mathrm{A}_{2 \mathrm{~A}} \mathrm{R}$ antagonists may reduce ethanol-induced stellate cell activation and fibrosis (Szuster-Ciesielska et al., 2012; Chiang et al., 2013). MRS1754, an $\mathrm{A}_{2 \mathrm{~B}} \mathrm{R}$ antagonist, reduced hepatic collagen deposition during fibrosis progression (Stoll et al., 2012).

Activation of $\mathrm{P}_{2} \mathrm{Y}_{2}$ and $\mathrm{P}_{2} \mathrm{Y}_{6} \mathrm{R}$ regulates procollagen-1 transcription and may be targets to treat or prevent liver fibrosis, thereby avoiding cirrhosis and chronic liver failure. PPADS, a P2R antagonist, inhibited HSC proliferation and prevented the development of non-biliary liver fibrosis (Dranoff et al., 2007). NTPDase 2 is a preferential ATPase that greatly influences inflammation and biliary type fibrogenesis. Ecto-5' ${ }^{\prime}$-nucleotidase (CD73) gene expression in HSC and portal fibroblasts increased during myofibroblastic differentiation and is a therapeutic target for antifibrotic therapy (Fausther et al., 2012).

Human platelet ATP release contributes to reduced type I collagen production and HSC activation in vitro (Ikeda N. et al., 2012). In progressive fibrosis, eicosapentaenoic acid replenishes hepatic levels of ATP leading to a reduction of steatosis and inflammation (Jia et al., 2012). Blockade of the P2X7R-NLRP3 inflammasome axis is considered to be a potential therapeutic target for liver fibrosis (Jiang et al., 2017).

\section{Cirrhosis}

Liver injury induced by alcohol is associated with enhanced inflammatory responses and adenosine, acting via $A_{2 A} R$, may prove to be an effective strategy for reducing liver injury (Pritchard et al., 2011). During fulminant hepatitis, $\mathrm{A}_{2 \mathrm{~A}} \mathrm{R}$ contribute to the anti-inflammatory actions that limit liver damage (Choukèr et al., 2008). In cirrhosis, platelet dysfunction 
is partly mediated by purinergic signalling. Cerebral $A_{1} R$ are involved in liver cirrhosis (Boy et al., 2008). Ectonucleotidase NTPDase 2 is down-regulated in biliary cirrhosis. It has also been claimed that endogenous $\mathrm{A}_{1} \mathrm{R}$ activation may protect mice against liver injury induced by acute ethanol by reducing oxidative stress and decreasing the accumulation of lipid (Yang et al., 2013). ATP- $\mathrm{MgCl}_{2}$ was used early to improve survival following massive hepatectomy in cirrhotic rats and adenosine partially reversed cirrhosis induced by carbon tetrachloride. In alcoholic liver disease, ATP and uric acid mediate inflammatory cross-talk between immune cells and hepatocytes (Petrasek et al., 2015). $A_{2 A} R$ are involved in the pathogenesis of hepatic cirrhosis (Chan et al., 2006).

\section{Hepatitis}

In vitro infection by duck hepatitis B virus, Rous sarcoma virus, and hepatitis delta virus was inhibited by suramin. Sympathetic nerves influence immune-mediated experimental hepatitis (Neuhuber and Tiegs, 2004) and ATP released as a cotransmitter with NA is probably involved. In autoimmune hepatitis P2X7R regulate NKT cells (Kawamura et al., 2006). P2X7R activation participates in hepatitis delta and hepatitis B virus infection of human hepatocytes (Taylor and Han, 2010). Leptin-induced GLUT4 function in stellate cells in non-alcoholic steatohepatitis is mediated by P2X7R (Chandrashekaran et al., 2016). Chronic hepatitis $C$ virus infection evokes progressive liver disease, exhibiting cirrhosis, insulin resistance, fibrosis and finally liver cancer. P2X4 and P2X7R may be a major component of the purinergic signalling complex in hepatitis $C$ virus-induced liver pathogenesis (Manzoor et al., 2016). In rats, $\mathrm{A}_{2 \mathrm{~A}} \mathrm{R}$ stimulation inhibited hepatocyte lipotoxicity and non-alcoholic steatohepatitis (Imarisio et al., 2012). Adenosine, via $A_{2 A} R$, controls NKT-cell-dependent hepatitis induction (Subramanian et al., 2014). Purinergic mechanisms involved in autoimmune hepatitis have been reviewed (Kapila et al., 2013).

\section{Liver Transplantation}

Human liver can be maintained successfully under hypothermic conditions for a maximum of $10 \mathrm{~h}$ using adenosine at high concentrations, although overcoming ischaemic damage is a major obstacle. However, infusion of ATP can preserve cells injured sublethally by enhancing their recovery following ischaemic injury and purinergic receptor antagonists prevent cold preservation-induced cell death. Upregulation of CD39 post-adenoviral infection prolongs transplant graft survival. After transplantation regeneration of the donor liver is important and ATP, via $\mathrm{P} 2 \mathrm{Y}_{2} \mathrm{R}$, activates hepatocyte cell cycle progression and proliferation in vitro and modulates growth factor activities in vivo (Thevananther et al., 2004). $\mathrm{A}_{2 \mathrm{~A}} \mathrm{R}$ stimulation down regulated adhesion molecules, proinflammatory cytokines and ultimately improved liver function following liver transplantation in rats (Tang et al., 2010). In post-transplant allografts, purinergic signalling is used to predict as well as monitor progression of fibrosis and rejection. Blood from acute rejection patients showed raised intracellular levels of ATP in $\mathrm{CD}^{+}{ }^{+}$lymphocytes (Qu et al., 2017).

\section{Liver Cancer}

Primary liver malignant tumours are subdivided in to hepatocarcinoma, bile duct carcinoma (cholangiocarcinoma) and hepatocholangiocarcinoma. ATP increases calcium uptake by rat hepatoma cells, probably via $\mathrm{P}_{2} \mathrm{Y}_{2}$ or $\mathrm{P}_{2} \mathrm{Y}_{4} \mathrm{R}$ subtypes. There is upregulation of $\mathrm{P} 2 \mathrm{Y}_{2} \mathrm{R}$ in human hepatocellular carcinoma cells (Tak et al., 2016), which mediates proliferation and migration of the cells (Xie et al., 2014). Carcinoma-specific expression of $\mathrm{P}_{2} \mathrm{Y}_{11} \mathrm{R}$ make a major contribution to ATP-induced signalling that controls cell migration in human hepatocellular carcinoma cells (Khalid et al., 2017). CD39 KO mice showed an increased incidence of spontaneous and induced hepatocellular carcinoma. Intra-arterial injection of an inhibitor of ATP production was suggested as a novel liver cancer therapy. The effects of ATP infusions in vivo on rat hepatocarcinomas have been investigated (Frontini et al., 2011). P2X3R over-expression is involved in poor recurrence-free survival in hepatocellular carcinoma patients and identifies the P2X3R as a potential therapeutic target (Maynard et al., 2015). Inhibition by adenosine of hepatoma cell growth was reported early.

The $\mathrm{A}_{3} \mathrm{R}$ agonist, $\mathrm{CF} 101$, inhibited liver metastasis (following colon carcinoma) (Bar-Yehuda et al., 2008). CF102, another selective $\mathrm{A}_{3} \mathrm{R}$ agonist, has anti-inflammatory and anti-tumour effects on the liver and was studied in a clinical trial for hepatocellular carcinoma patients (Stemmer et al., 2010). $\mathrm{A}_{2 \mathrm{~B}} \mathrm{R}$ are strongly expressed in human hepatoma cellular carcinoma (Xiang et al., 2006). Mouse regulatory T cell CD39 expression mediated inhibition of NK cell activity and promoted hepatic metastatic tumour growth (Sun et al., 2013). Liver metastasis from colorectal cancer is one of the main causes of cancer-related morbidity and ATP-chemotherapy may effectively treat initially unresectable colorectal liver metastasis (Hur et al., 2012). In hepatocellular carcinoma, up-regulation of ATP-binding cassette transporter genes is mediated by cellular microRNAs (Borel et al., 2012).

\section{DISEASES OF THE REPRODUCTIVE SYSTEM}

Reviews are available about purinergic signalling in the reproductive system in both health and disease (Burnstock, 2014c; Gorodeski, 2015).

\section{Disorders of the Male Reproductive Tract Erectile Dysfunction}

Abnormalities in purinergic signalling, including impaired ATPmediated cavernosal relaxation, may contribute to erectile dysfunction associated with prostate enlargement and diabetes and may provide a target for therapy (Hupertan et al., 2012; Wen and Xia, 2012). Normal penile erectile function involves a fine balance between contraction and relaxation in the corpus cavernosum smooth muscle. The strong relaxation induced by ATP via P2YR on human corpus cavernosum is comparable to that produced by NO, thus ATP together with an NO donor may prove to be effective for erectile disorders (Hupertan et al., 2012). ATP released from sympathetic nerves leads to relaxation 
of cavernosum smooth muscle via $\mathrm{P}_{2} \mathrm{Y}_{4} \mathrm{R}$, whereas $\mathrm{ADP}$, after breakdown of ATP released from endothelial cells, acts via $\mathrm{P} 2 \mathrm{Y}_{1} \mathrm{R}$ on endothelial cells to produce relaxation via NO (Calvert et al., 2008a). P2XR might also be involved (Gur et al., 2007; Phillips et al., 2014).

In anaesthetised dogs, ATP and adenosine induce penile tumescence, probably via $\mathrm{A}_{2} \mathrm{R}$; pelvic nerve stimulation also produced tumescence. Impaired adenosine signalling via $A_{1} R$ contributes to erectile dysfunction (Ning et al., 2012a). Elevated adenosine signalling, via $\mathrm{A}_{2 \mathrm{~B}} \mathrm{R}$, may contribute to priapism, where there is persistent penile erection lasting at least $4 \mathrm{~h}$ without sexual excitation (Dai et al., 2009; Ning et al., 2012b). A review highlights adenosine signalling in penile tissue as a potential therapeutic target to treat erectile disorders (Wen and Xia, 2012). Impaired erectile function occurs in CD73 KO mice resulting in decreased endogenous adenosine. Corpus cavernosum from men suffering from vasculogenic erectile dysfunction show lower ectonucleotidase CD39 activity resulting in ATP accumulation. Human corpus cavernosum relaxation by P2R agonists was substantially reduced in erectile dysfunction patients (Faria et al., 2010). ATP release from cavernosal tissue increased in patients following prostatectomy. Activating ATP-mediated pathways may restore erectile function in diabetics where there is impaired NO bioavailability. It has been suggested that P2X3 antagonists may improve recovery of erectile function (Li C.L. et al., 2015).

\section{Male Fertility and Contraception}

ATP increases the fertilising potential of sperm in humans and is used to treat spermatozoa during in vitro fertilisation. In P2X1R KO mice fertility was diminished with decreased number of spermatozoa in the ejaculate (Mulryan et al., 2000). This raises the possibility that $\mathrm{P} 2 \mathrm{X} 1 \mathrm{R}$ antagonists would provide a safe and effective contraceptive (White et al., 2013). In rat testes, during spermatogenesis there is differential, stagedependent immunostaining for P2XR subtypes (Glass et al., 2001), suggesting purinergic targets for both fertility and contraception. ATP triggers the acrosome reaction via P2X7R in human spermatozoa (Torres-Fuentes et al., 2015). Purinergic signalling plays a role in the maturation of sperm cells in the testes. When the selective P2X1 and P2X3R desensitiser, $\alpha, \beta$-meATP, was injected into the cauda epididymis, fertility in male rats was impaired. Adenosine stimulates human sperm motility via $\mathrm{A}_{2} \mathrm{R}$.

\section{Prostatic Hyperplasia}

Purinergic compounds have been suggested for the therapeutic treatment of benign prostatic hyperplasia (Andersson et al., 2002). Injection of BTX into the prostate, which reduces ATP and $\mathrm{ACh}$ release, treats bladder obstruction hyperactivity, by decreasing prostate size and as such improving urine flow rate (Chuang and Chancellor, 2006).

\section{Disorders of the Female Reproductive Tract}

Regulating the proliferation of ovarian granulosa cells as well as steroidogenesis contributes to ovarian pathophysiology, since in rats with polycystic ovarian syndrome, theca hyperplasia occurs (Salvetti et al., 2009). The hydrolysis of ADP and ATP was reduced by ovariectomy and oestradiol replacement therapy (Pochmann et al., 2004). Ovarian tumours arise largely from the surface of squamous-to-cuboid mesothelium covering the ovary. ATP stimulated mitogen-activated kinase in neoplastic and pre-neoplastic surface epithelium, suggesting that co-released ATP from sympathetic nerves may contribute to the regulation of cell proliferation in neoplastic epithelial cells from the surface of the ovary (Choi et al., 2003).

In the human fallopian tube, ATP-mediated contractions are increased during acute purulent inflammation, probably as a result of upregulation of P2X1 and P2X2R (Ziganshin et al., 2008). It has been claimed that targeting $P 2 X 7 R$ may lead to new treatments to prevent uterine contractions in preterm deliveries. A naturally occurring P2X7 splice variant, the P2X7jR, blocks P2X7R-mediated actions (Feng et al., 2006). It is co-expressed with P2X7R in female reproductive tract epithelia. The P2X7j isoform hetero-oligomerises with the $\mathrm{P} 2 \mathrm{X} 7 \mathrm{R}$ and co-expression of P2X7R and P2X7jR blocks ATP-induced pore formation, and abolishes agonist-induced apoptosis. $\mathrm{P} 2 \mathrm{Y}_{2} \mathrm{R}$ agonists may be a non-hormonal alternative therapy for treating vaginal dryness in post-menopausal women. ATP is considered as a therapeutic target to control uterine activity during difficult labours (Zafrah and Alotaibi, 2017).

The P2X7R contributes to the control of cervical infections. P2X7R-mediated activation of cervical epithelial cells inhibits Chlamydia and mycobacteria infection (Darville et al., 2007). ATP regresses endometriosis in a rat model (Zhang C. et al., 2016). Adenosine in the placenta mediates the placental disturbances induced by alcohol, perhaps contributing to the pathogenesis of foetal alcohol syndrome (Acevedo et al., 1997). Adenosine protects vaginal epithelial cells from $T$. vaginalis cytotoxicity (Menezes and Tasca, 2016). Plasma adenosine is raised in hyperemesis gravidarum (severe morning sickness) and serves as a prejunctional modulator of sympathetic neurotransmission, which limits further progression of this pregnancy-related disease (Yoneyama et al., 2004).

\section{Preeclampsia}

ATP infusion in pregnant rats involved an inflammatory response that occurs in preeclampsia (Spaans et al., 2014a). Elevated placental adenosine signalling contributes to the pathogenesis of preeclampsia (Iriyama et al., 2015). Hypoxia stimulates ATP release, which is rapidly broken down to adenosine by ectonucleotidases and women with preeclampsia and their foetuses have increased circulating adenosine concentrations. There is elevation of adenosine $\mathrm{A}_{2 \mathrm{~A}} \mathrm{R}$ expression in placental biopsies, villous explants and placental microvillous membranes (von Versen-Höynck et al., 2009). In preeclampsia $\mathrm{A}_{2 \mathrm{~B}} \mathrm{R}$ on microvascular endothelial cells have also been implicated (Escudero et al., 2008). Reduced adenosinemediated angiogenesis in preeclamptic pregnancies may be associated with hypertension development in the offspring (Escudero et al., 2014). The interaction between $A_{2 A} R$ and the angiotensin system may be involved in the early growth of the placenta. $\mathrm{A}_{2 \mathrm{~A}} \mathrm{R}$ expression is raised in pre-eclampsia, perhaps 
as a consequence of poor placental perfusion in preeclampsia (Kurlak et al., 2015). Elevation of ADA activity in women with preeclampsia may contribute to their increased levels of uric acid and pro-inflammatory immune activity (Giorgi et al., 2016). Release of ATP increases in preeclampsia following hypoxia and oxidative/nitrative stress, which acts on P2X4R to influence homeostasis of the placenta (Roberts et al., 2007). There is deficient spiral artery remodelling and trophoblast invasion in preeclampsia, both of which may be inhibited by ATP-induced activated macrophages (Spaans et al., 2014b). It was concluded from a study of the relationship between the foeto-placental adenosine release and utero-placental circulatory insufficiency in pregnancies featuring preeclampsia, that foetal plasma adenosine increases before utero-placental insufficiency induces generalised foetal hypoxia (Yoneyama et al., 1996).

\section{Malignant Cancer of Reproductive Organs \\ Prostate Cancer}

Prostate cancer is the second most common male cancer and the third leading cause of cancer death. Prostate cancer cells are sensitive to extracellular ATP. ATP and adenosine inhibit the growth of human prostate cancer cells (Lertsuwan et al., 2017), identified at that time to act via $\mathrm{P} 2 \mathrm{Y}_{1}, \mathrm{P}_{2} \mathrm{Y}_{2}$ and/or $\mathrm{P}_{2} \mathrm{Y}_{4}, \mathrm{P}_{2} \mathrm{Y}_{6}$ and $\mathrm{P} 2 \mathrm{Y}_{11} \mathrm{R}$ subtypes. Activation of $\mathrm{P} 2 \mathrm{Y}_{1} \mathrm{R}$ inhibited growth and induced cell death of prostate cancer PC-3 cells and $\mathrm{P}_{2} \mathrm{Y}_{1} \mathrm{R}$ agonists were claimed to be therapeutically promising for prostate cancer (Wei et al., 2011).

However, prostate tumour cells were shown later to also express P2X4, P2X5, and P2X7R in PC-3 cells and P2X4 and P2X5R in DU145 cells. ATP inhibited tumour cell growth, but not by UTP or adenosine, while $2^{\prime}\left(3^{\prime}\right)$-O-(4-benzoylbenzoyl) ATP increased apoptotic cell death in PC-3 cells, probably via P2X7R. CD73 KO mice resist prostate carcinogenesis and CD73 promoted de novo prostate tumorigenesis. Anti-CD73 monoclonal antibodies decreased tumour growth and metastasis in the prostate (Stagg et al., 2012).

There are many polymorphisms of the P2X7R (Fuller et al., 2009), which, as well as resulting in loss of function, alter receptor activity. Cytolytic P2X7R expression was found in 116 prostate cancer pathology specimens (Slater et al., 2004). In normal tissues from patients with no evidence of cancer, P2X7R were not expressed, suggesting that the appearance of P2X7R is an early marker of prostate cancer.

Prostate tumour cell proliferation is inhibited by adenosine. $\mathrm{A}_{3} \mathrm{R}$ activation by IB-MECA inhibited proliferation of prostate cancer cells and induced cell cycle arrest and apoptosis (Aghaei et al., 2012). Activation of $A_{3} R$ suppressed prostate cancer metastasis (Jajoo et al., 2009). The ATP synthase $\beta$ subunit also plays a role in prostate cancer metastasis (Li W. et al., 2017).

\section{Breast Cancer}

Inhibition of growth of human breast cancer cells by ATP was shown for the first time in 1993. Chemotherapeutic ATP release from breast tumour cells of mice increased tumour regression through apoptosis and it was suggested that $\mathrm{P}_{2} \mathrm{Y}_{2}$ and/or $\mathrm{P}_{2} \mathrm{Y}_{4} \mathrm{R}$ were involved. Oestrogen, acting via oestrogen receptor $\alpha$, promoted proliferation of breast cancer cells by downregulating expression of $\mathrm{P}_{2} \mathrm{Y}_{2} \mathrm{R}$ and reducing $\mathrm{P} 2 \mathrm{Y}_{2} \mathrm{R}$-induced increase in $\left[\mathrm{Ca}^{2+}\right]_{\mathrm{i}}$ ( $\mathrm{Li}$ et al., 2011). $\mathrm{P} 2 \mathrm{Y}_{2} \mathrm{R}$ activation by ATP released from cancer cells induces the invasion of metastatic breast cancer cells (Eun et al., 2015). Up-regulation of $\mathrm{P} 2 \mathrm{Y}_{6} \mathrm{R}$ occurs in the mesenchymal phenotype of breast cancer cells and inhibition of $\mathrm{P}_{2} \mathrm{Y}_{6} \mathrm{R}$ may be a useful therapeutic target for metastasis of breast cancer (Azimi et al., 2016; Ma X. et al., 2016).

Antibody therapy with anti-CD73 inhibited breast tumour growth and metastasis (Stagg et al., 2010). Bisphosphonates are also effective inhibitors of breast cancer (Fehm et al., 2012). Proteomic analysis of human breast carcinoma revealed upregulation of ATP synthase in tumours and the ATP synthase inhibitor, aurovertin B, inhibited proliferation of several breast cancer cell lines (Huang et al., 2008). Malignant breast carcinoma cells release ATP making the pre-metastatic environment suitable for micro-metastasis in lymph nodes and associated afferent lymph vessels (Kawai et al., 2008).

In breast tumour cells ATP increased $\left[\mathrm{Ca}^{2+}\right]_{\mathrm{i}}$ and high concentrations produced apoptosis via P2X7R. Activation of $\mathrm{P} 2 \mathrm{X} 7 \mathrm{R}$ in the human breast cancer cell line, T47D, increased cell migration and development of metastases, suggesting that P2X7R antagonists may have therapeutic roles (Xia et al., 2015). The role of hypoxia in the regulation of tumour progression has been debated. However, P2X7R expression is increased by hypoxia and hypoxia-driven increase in $\mathrm{P} 2 \mathrm{X} 7 \mathrm{R}$ enhances tumour cell invasion and migration. Silencing of P2X5R inhibited cell proliferation and may be a new mechanism to target cancer metastasis.

$A_{1}$ and $A_{3} R$ mRNA are expressed on human breast tumours (Panjehpour et al., 2012). Adenosine induces tumour cell proliferation and migration of T-47D and MCF-7 breast carcinoma cell lines (Mujoomdar et al., 2004). MDA-MB231, a human breast cancer cell line, expressed $A_{2 B} R$, which mediated cell proliferation and $\mathrm{A}_{2 \mathrm{~B}} \mathrm{R}$ inhibition slowed growth of breast tumours (Cekic et al., 2012). $A_{3} R$ agonists reduced bone metastasis of breast cancer, suggesting a therapeutic approach to bone-residing breast cancer (Varani et al., 2013).

\section{Cervical Cancer}

HeLa cells from human cervical cancer are used to study purinergic signalling involvement in cancer. Activation of $\mathrm{P} 2 \mathrm{Y}_{2} \mathrm{R}$ with UTP and ATP caused proliferation of HeLa cells. P2Y 4 and $\mathrm{P}_{2} \mathrm{Y}_{6} \mathrm{R}$ expression increased during proliferation. Stimulation of $\mathrm{P} 2 \mathrm{Y}_{1} \mathrm{R}$ on HeLa cells triggered epidermal growth factor receptor mitogen signalling and $\mathrm{P}_{2} \mathrm{Y}_{1}$ antagonists reduced proliferation. Permeabilisation of cervical cancer cells to a cytotoxin is activated by P2YR (Bukhari et al., 2015). P2Y 6 R activation induces HeLa cell migration (Gendaszewska-Darmach and Szustak, 2016). Oestrogen reversed the apoptotic activity mediated by P2X7R in normal human cervix, but not in cervical epithelial cancer cells. A truncated P2X7R variant (P2X7-j), expressed in cervical cancer cells, antagonised the P2X7R through hetero-oligomerisation. Gentle mechanical stimulation released ATP from HeLa cells. The presence of ectonucleotidase in human cervical cancer cell regulates the levels of nucleotides, limiting their effects (Beckenkamp et al., 2014). 


\section{Ovarian Cancer}

ATP raised $\left[\mathrm{Ca}^{2+}\right]_{i}$ and stimulated growth of SKOV-3 and OVCAR-3 human ovarian carcinoma cells. ATP may act as a messenger to control the ovarian epithelial cell cycle through $\mathrm{P} 2 \mathrm{Y}_{2} \mathrm{R}$ on human ovarian cancer cells. $\mathrm{A}_{2} \mathrm{R}$ antagonists inhibited angiogenic activity of human ovarian cancer cells. The treatment of human ovarian carcinoma with cisplatin in the presence of ATP results in additive cytotoxicity (Rotte et al., 2010). P2X7R were highly expressed in both human ovarian tumours and ovarian cancer cell lines and paracrine release of ATP acts on P2X7R to cause proliferation of ovarian cancer cells (VazquezCuevas et al., 2014).

\section{Uterine Cancer}

$\mathrm{P} 2 \mathrm{Y}_{2} \mathrm{R}$ play a role in the control of the cell cycle and in the suppression of proliferation of human endometrial carcinoma cells. The P2X7R has been used as a biomarker for uterine epithelial cancers. There is decreased P2X7R expression on endometrial epithelium in pre-cancerous and cancer cells (Li et al., 2007, 2009). Activation of P2X7R-dependent apoptosis has a chemotherapeutic growth-preventive effect on pre-cancerous and early cancerous epithelial lesions ( $\mathrm{Fu}$ et al., 2009; Gorodeski, 2009). Loss of CD73 in endometrial cancer allows for tumour progression (Bowser et al., 2016). A higher proportion of ADA2*1/*1 genotype was observed in women with uterine leiomyomas (Gloria-Bottini et al., 2016).

\section{SKIN DISEASES}

Reviews about purinergic signalling in the skin during health and disease have been published (Burnstock et al., 2012b; Geraghty et al., 2016). Changes in P2R subtype expression occurs in the epidermis during proliferative disorders, such as psoriasis and scleroderma, and $\mathrm{P}_{2} \mathrm{Y}_{2} \mathrm{R}$ may be a novel target to treat these disorders. ATP plays an important role in wound healing, the defence response, innate immunity and inflammation in the skin and might be an important therapeutically in psoriasis and scleroderma.

\section{Psoriasis}

Psoriasis is a chronic skin disease involving epidermal hyperproliferation. ATP and parathyroid hormone-related protein increased proliferation in $\mathrm{HaCaT}$ cells and may account for the hyperproliferation that occurs in psoriasis. $\mathrm{P} 2 \mathrm{Y}_{2} \mathrm{R}$ contribute to epidermal homeostasis and indicate a possible therapy for psoriasis. $\mathrm{P} 2 \mathrm{Y}_{11} \mathrm{R}$ mediate IL-6 production in human keratinocytes, which is important in psoriasis (Ishimaru et al., 2013). P2X7R signalling induces inflammation leading to differentiation of Th17 lymphocytes, which are involved in the pathogenesis and potential treatment of psoriasis (Killeen et al., 2013). P2X7R play a role in shaping the inflammatory microenvironment in psoriasis (Lioi et al., 2015). Strong P2X7R expression is confined to the basal layer cell membrane, while $\mathrm{P} 2 \mathrm{Y}_{1} \mathrm{R}$ were expressed all through the psoriatic epidermis.

In the blood of psoriasis patients, there are high levels of adenosine. Adenosine raised cyclic AMP levels in lesions of epidermis from psoriasis patients. There is a defective purine nucleotide synthetic pathway in patients with psoriasis and nucleotide metabolism is altered in psoriatic keratinocytes. In psoriasis patients, ADA levels were significantly elevated in both serum and epidermis. It was concluded from a clinical trial that caffeine, a P1R antagonist, is an inexpensive, safe and effective treatment for psoriasis. The role of adenosine as an endogenous mediator of the pathogenesis of psoriasis has been reviewed (Festugato, 2015) and clinical trials for the use of $A_{3} R$ agonists for the treatment of psoriasis described (Borea et al., 2015; Kofoed et al., 2015). $A_{2 A} R$ are upregulated in psoriasis and $A_{2 A} R$ agonists may counteract inflammation in this disease (Merighi et al., 2017). $\mathrm{A}_{2 \mathrm{~B}} \mathrm{R}$ are also expressed by human epidermal keratinocytes and their expression is reduced in psoriasis (Andrés et al., 2017).

\section{Scleroderma}

Scleroderma encompasses a spectrum of disorders that cause dermal fibrosis and systemic sclerosis (SSc). SSc is a severe disease of the connective tissue of affected organs, including the skin. Adenosine $\mathrm{A}_{2 \mathrm{~A}} \mathrm{R}$ contribute to the pathogenesis of dermal fibrosis and may be a therapeutic target to treat and prevent dermal fibrosis in scleroderma (Chan and Cronstein, 2010). Fibroblasts from SSc patients had a high rate of spontaneous IL-6 release, which was increased by ATP stimulation. SSc fibroblasts expressed mRNA for P2X3, P2X4, P2X7, $\mathrm{P} 2 \mathrm{Y}_{1}, \mathrm{P}_{2} \mathrm{Y}_{2}, \mathrm{P}_{2} \mathrm{Y}_{4}$, and $\mathrm{P}_{2} \mathrm{Y}_{6}$, suggesting a possible therapeutic role for $\mathrm{P} 2 \mathrm{R}$ antagonists in SSc patients via modulation of fibroblast function.

\section{Skin Inflammation}

The skin is an immune defence organ. Chemical, immunespecific or physical insults evoke increased expression of proinflammatory mediators, in particular keratinocytes release chemokines. Release of ATP is associated with inflammation of the skin, as well as increase in expression of, in particular, P2X7 and perhaps $\mathrm{P}_{2} \mathrm{Y}_{1} \mathrm{R}$, and subsequent release of proinflammatory cytokines. Skin inflammation is reduced by P2X7R antagonists.

In ectonucleotidase (CD39) KO mice, rapid ATP release from keratinocytes is triggered by irritant chemicals causing exacerbated skin inflammation. Ultraviolet (UV) radiation evokes ATP release from keratinocytes and $\mathrm{P}_{2} \mathrm{Y}_{6} \mathrm{R}$ mediate UV radiation-induced inflammatory responses (Takai et al., 2011). ATP, when released after trauma or infection, may enhance immunoresponses and $\mathrm{P} 2 \mathrm{R}$ agonists may increase vaccine efficacy. $A_{1}$ and $A_{2} R$ may function as cutaneous neurogenic pro-inflammatory mediators. Human skin keratinocytes infected by Staphylococcus aureus, which releases $\alpha$-toxin, exhibited a transient reduction in cellular ATP levels (Suriyaphol et al., 2009). $\mathrm{P} 2 \mathrm{X} 3$ and $\mathrm{P} 2 \mathrm{X} 2 / 3$ nociceptive receptors on sensory nerve endings are increased in inflamed skin and antagonists to these receptors have been developed as analgesics.

\section{Wound Healing}

Topically applied $\mathrm{A}_{2 \mathrm{~B}}$ and particularly $\mathrm{A}_{2 \mathrm{~A}} \mathrm{R}$ agonists promote cutaneous wound healing in both healthy and diabetic conditions. $\mathrm{A}_{2 \mathrm{~A}} \mathrm{R}$ agonists also promote collagen production by 
dermal fibroblasts. Adenosine inhibits proliferation of vascular smooth muscle cells via $\mathrm{A}_{2} \mathrm{R}$ activation. A review discusses the involvement of $\mathrm{A}_{2 \mathrm{~A}}$ and $\mathrm{A}_{2 \mathrm{~B}} \mathrm{R}$ signalling in wound healing and fibrosis (Shaikh and Cronstein, 2016).

ATP and ADP also play a role in wound healing. ATP, released by damaged cells and physiologically during gentle mechanical stimulation, contributes to wound healing, tissue repair and regeneration. Cutaneous wound healing is accelerated by Mg-ATP, probably by increasing synthesis of vascular endothelial growth factor. Release of ATP from platelets and other cells during wound healing results in an increase in $\left[\mathrm{Ca}^{2+}\right]_{\mathrm{i}}$ in keratinocytes, associated with epidermal growth and differentiation. ATP improves ischaemic skin flap survival after surgery, while $\mathrm{P} 2 \mathrm{R}$ antagonists accelerate barrier repair.

There is acute inflammation in the initial phase of wound healing. ATP is involved in the development of inflammation through P2X7R-mediated production and release of cytokines from immune cells. The presence of P2X7R on immune cells mediates killing of intracellular pathogens by stimulating apoptosis of the host macrophage, chemo-attraction and cell adhesion. In contrast, adenosine has anti-inflammatory effects. ATP released following infection and trauma acts as an endogenous adjuvant to increase the immune response and P2R agonists may enhance the efficacy of vaccines.

Wound healing also involves new vessel growth (angiogenesis) and both ATP and adenosine contribute to cell proliferation and migration during angiogenesis. In cultured porcine artery smooth muscle cells, ADP and ATP stimulate DNA synthesis and cell proliferation. ATP and UTP are mitogenic in human vascular smooth muscle cells and $\mathrm{P}_{2} \mathrm{Y}_{2}$ and $\mathrm{P} 2 \mathrm{Y}_{4} \mathrm{R}$ might be involved.

Wound healing is delayed in denervated wounds. In a rat model of this, $\mathrm{P} 2 \mathrm{X} 5, \mathrm{P} 2 \mathrm{X} 7, \mathrm{P}_{2} \mathrm{Y}_{1}$, and $\mathrm{P} 2 \mathrm{Y}_{2} \mathrm{R}$ expression was altered in the epidermis. $\mathrm{P} 2 \mathrm{Y}_{1} \mathrm{R}$ expression was increased in the basal proliferating layer of keratinocytes in the regenerating epidermis, while $\mathrm{P}_{2} \mathrm{Y}_{2} \mathrm{R}$ were significantly decreased. ATP and UTP, probably via $\mathrm{P} 2 \mathrm{Y}_{2} \mathrm{R}$, increased proliferation of the MCS-P5 murine keratinocyte cell line, and enhanced wound healing in mice (Kehasse et al., 2013; Jin et al., 2014). ATP release and P2YR signalling mediate electric field-stimulated directional keratinocyte migration (Riding and Pullar, 2016). A review discussed the clinical applications of purinergic compounds to enhance wound healing (Gendaszewska-Darmach and Kucharska, 2011).

\section{Warts}

Warts are caused by human papillomavirus infection of epidermis basal keratinocytes. P2X5R staining was increased in warts. P2X7R immunoreactivity was found in hyperkeratotic areas of the stratum corneum and in nuclei of koilocytes in the wart suprabasal layers. The nuclei positive for P2X7R were shrunken, showing much more intense P2X7R staining. The expression of P2X7R in the nucleus of human papillomavirusinfected cells was associated with disruption of the cellular machinery. P2X7R agonists may be used to trigger apoptosis in these virally infected cells. P2X5 and P2X7R are being explored for the treatment of warts.

\section{Allergy}

Contact allergen sensitisation involves immune system activation by endogenous danger signals. P2X7R KO mice exhibit resistance to contact hypersensitivity. P2X7R KO dendritic cells do not induce sensitisation in response to contact allergens or release IL- $1 \beta$ in response to ATP. This suggests that P2X7R are crucial for release of ATP from skin in response to contact allergens. Interference with $\mathrm{P} 2 \mathrm{X} 7 \mathrm{R}$ signalling could be a therapeutic approach to prevent allergic contact dermatitis.

\section{Hailey-Hailey and Darier Diseases}

These are autosomal dominant skin disorders and are characterised by epidermal keratinocyte dissociation (acantholysis) at the epidermis suprabasal layer. In lesions P2 $\mathrm{Y}_{2} \mathrm{R}$ are not localised on acantholytic cells, but P2X7R appear in the plasma membranes, potentially mediating apoptosis.

\section{Barrier Function}

The skin protects the water-rich internal organs from environmental dryness, with the stratum corneum being critical to the water-impermeable barrier. Topical application of ATP and $\alpha, \beta$-meATP via P2XR delayed barrier recovery, damaged by surfactant, organic solvent or tape stripping. P2R antagonists, however, accelerated barrier repair. In mice skin wounds healed faster following treatment with ATP-encapsulated fusogenic lipid vesicles rather than with lipid vesicles. PPADS, a P2XR antagonist, accelerates skin barrier repair and prevented epidermal hyperplasia.

\section{Burns}

There is increased concentration of adenosine in burn blister fluid, and depletion of ATP in skin after thermal injury. ATP has a protective effect against skin burns. ATP- $\mathrm{MgCl}_{2}$ administration following burn injury reversed the damage to the intravascular clearing of lipid emulsions within the reticuloendothelial immune system. Glucose metabolism was affected by ATP in thermally injured skin.

Burn injury produces severe pain and the relieving effects of tetramethylpyrazine, a Chinese medicine, are claimed to be due to it acting as a P2X3R antagonist (Gao Y. et al., 2010). Skin P2X3R expression was increased on sensory nerve terminals in first and second degree burns, but, following treatment with tetramethylpyrazine, P2X3R expression was reduced. P2X3R may play a role in the short-lasting thermal hyperalgesia induced by mild heat injury (Füredi et al., 2010). After a superficial skin burn, adenosine reduced the skin area showing hypersensitivity.

\section{Pain}

Skin pain is associated with changes in sympathetic nerve activity and ATP released from rat sympathetic nerves as a cotransmitter with NA may modulate cutaneous nociceptors. P2X3R are expressed on sensory neurons in DRG, nodose and trigeminal ganglia that innervate cutaneous tissues. ATP and ADP stimulate skin afferent nerve terminals and skin cell damage stimulates nociceptive sensory nerves via ATP release. In rat skin with carrageenan-induced inflammation, nociceptors can be 
selectively activated with $\alpha, \beta$-meATP, indicating the involvement of P2X3R. ATP contributes to the enhanced sensitivity of inflamed skin nociceptors, leading to heat hyperalgesia. P2X3R antagonists are anti-nociceptive. UV light evokes hypersensitivity of skin to ATP-induced pain. After subcutaneous bee venom injection, there is reduction of prolonged pain by PPADS, and it was suggested that stimulation of P2XR in the spinal cord contributes to cutaneous pain. The terminals of nociceptive neurons in the skin are targets for P2X3R antagonists. UTP sensitises a subpopulation of cutaneous C-fibre nociceptors to mechanical stimuli. There is increased P2X3R expression on calcitonin gene-related peptide-positive sensory fibres during the growth of tumours, mediating nociception. Increased expression of skin glial cell neurotrophic factor increases the mechanical sensitivity of nociceptive afferents expressing P2X3R.

Ecto-5'-nucleotidase (CD73) is located on nociceptive terminals and epidermal keratinocytes in the epidermis, which, by hydrolysing AMP to adenosine, is suggested to be antinociceptive. Blockade of peripheral $\mathrm{P} 2 \mathrm{Y}_{1} \mathrm{R}$ prevents thermal hyperalgesia (Kwon et al., 2014). Mechanical allodynia is induced by $\alpha, \beta$-meATP, which sensitises P2X3R on cutaneous nociceptive sensory fibres (Ren et al., 2015).

Reviews with coverage of purinergic signalling and cutaneous pain are available (Zhu and Lu, 2010; Burnstock, 2016c).

\section{Dermatitis}

In irritant dermatitis, there is a pathogenic role for keratinocytederived ATP. The necrosis produced by the chemical irritant croton oil was prevented by the pre-treatment with A438079, a selective P2X7R antagonist (Zanin et al., 2015). Topical application of $\mathrm{A}_{2 \mathrm{~A}}$ antagonists prevents radiation dermatitis (Perez-Aso et al., 2016).

\section{Skin Cancer}

Different subtypes of P1R and P2R are involved in skin cancer, playing roles in differentiation, apoptosis and proliferation. The roles of purinoceptors are complicated because there are multiple receptor subtypes on the same cell, which can have opposing effects. For example, $\mathrm{P} 2 \mathrm{Y}_{2} \mathrm{R}$ mediate an increase in tumour cell numbers, while P2 $\mathrm{Y}_{1}, \mathrm{P} 2 \mathrm{X} 5$, and $\mathrm{P} 2 \mathrm{X} 7 \mathrm{R}$ mediate a decrease in cell numbers. In cancers of the skin, proliferation outweighs apoptotic cell death.

UV light is a stimulus for the genesis of cutaneous cancer, including basal and squamous cell carcinoma as well as melanoma, where the UV-B component has the most severe effects. UV-B irradiation decreases the amount of $\mathrm{P} 2 \mathrm{X} 1, \mathrm{P}_{2} \mathrm{Y}_{2}$, and $\mathrm{P} 2 \mathrm{X} 7 \mathrm{R}$, which contributes to the malignant transformation of keratinocytes (Ruzsnavszky et al., 2011).

\section{Basal and Squamous Cell Carcinomas}

Basal cell and squamous cell carcinoma are tumours that generally occur after the age of 50, squamous cell carcinoma being the more common and aggressive of the two. Nucleoside analogues reduced basal cell carcinoma growth. A human cutaneous squamous cell (epidermal) carcinoma cell line, A431, express P2R that induce increases in $\left[\mathrm{Ca}^{2+}\right]_{\mathrm{i}}$. ATP-stimulated A431 cells induced production of inositol-trisphosphate, suggesting the involvement of P2YR. P2X5 and P2YR were strongly expressed on both squamous and basal cell carcinomas. $\mathrm{P} 2 \mathrm{X} 7 \mathrm{R}$ are expressed in apoptotic cells in superficial multifocal and infiltrative cells and in the necrotic centre of nodular basal cell carcinomas. Expression of $\mathrm{P} 2 \mathrm{Y}_{1} \mathrm{R}$ was confined to the stroma surrounding tumours. $\mathrm{P}_{2} \mathrm{Y}_{4} \mathrm{R}$ were present only in basal cell carcinomas. ATP and UTP at low concentrations induced an increase in the number of A431 cells, while high concentrations substantially decreased cell numbers.

ATP via P2X7R caused apoptosis of A431 cells and UTP and adenosine (after ATP breakdown) also induced cell death (Völkl et al., 2008). 2' (3')-O-(4-Benzoylbenzoyl) ATP, a potent P2X7R agonist, reduced skin carcinoma and papilloma and formation (Fu et al., 2009). ADA in saliva was shown to be a diagnostic marker of tongue squamous cell carcinoma (Rai et al., 2011).

\section{Melanoma}

Malignant melanoma, which is highly metastatic, is derived from melanocytes. ATP inhibited the growth of both human and animal melanoma cells in vivo. CD39 is over-expressed in differentiated human melanomas. Expression of P2X7R was increased in superficial spreading melanoma patients, which were later shown to be functional and may be a therapeutic target for melanoma therapy (White et al., 2005). A low pH environment as seen in solid tumours induced ATP release from B16 melanoma cells, which increases proliferation via P2X7R. Oxidised ATP, a P2X7R antagonist, inhibited tumour growth (Hattori et al., 2012). P2 $\mathrm{Y}_{1}, \mathrm{P} 2 \mathrm{Y}_{2}$, and $\mathrm{P} 2 \mathrm{Y}_{6} \mathrm{R}$ mRNA and protein expression was observed in human melanomas. The presence of $\mathrm{P}_{2} \mathrm{Y}_{1}$ and $\mathrm{P} 2 \mathrm{X} 7 \mathrm{R}$, which had been suggested to be therapeutic targets for melanoma treatment using ATP, was demonstrated by immunohistochemistry (White et al., 2009). The release of ATP from murine B16 melanoma cells was shown to upregulate CD39 expression on regulatory T cells. Dying tumour cells release ATP, which accumulates at high concentrations and acts as an immune danger signal, although it can also kill adjacent tumour cells directly via P2X7R (Feng et al., 2011). Accelerated melanoma tumour progression in mice lacking P2X7R has been reported (Adinolfi et al., 2015). $\gamma$-Irradiation that induces arrest in tumour cell growth and death, induced P2X7R-dependent release of ATP from B16 cells (Ohshima et al., 2010).

In an animal model of melanoma, tumour growth was reduced in CD73 KO mice (Yegutkin et al., 2011). Implanting B16 cells into CD73 KO mice, thereby decreasing the production of adenosine, resulted in reduced tumour growth (Ring et al., 2011). Adenosine potentiated the in vivo actions of chemotherapeutic agents. Antimetastatic therapies based on inhibition of $A_{1} R$ activation have been suggested. $A_{1}, A_{2 A}, A_{2 B}$ and $A_{3} R$ subtypes are expressed in the A375 human malignant melanoma cell line. $A_{3} R$ activation resulted in growth inhibition of melanoma cells (Morello et al., 2011). However, another study claimed that adenosine, acting through $\mathrm{A}_{3} \mathrm{R}$, induced cell proliferation of human malignant melanoma C32 cells (Soares et al., 2012). $\mathrm{A}_{2 \mathrm{~B}} \mathrm{R}$ antagonists impaired IL-8 production that is raised in malignant melanoma patients, while $\mathrm{A}_{3} \mathrm{R}$ antagonists decreased vascular endothelial growth factor that promotes human carcinoma cell angiogenesis and metastasis (Merighi et al., 2009). Adenosine is 
a potent immunoregulatory factor modulating cytotoxic activity and cytokine production of anti-melanoma specific T cells.

In conclusion, $\mathrm{P} 2 \mathrm{Y}_{2} \mathrm{R}$ mediate proliferation, $\mathrm{P} 2 \mathrm{X} 5 \mathrm{R}$ mediate differentiation (i.e., are antiproliferative) and P2X7R mediate cell death. Therefore, $\mathrm{P} 2 \mathrm{Y}_{2} \mathrm{R}$ antagonists and $\mathrm{P} 2 \mathrm{X} 5$ and $\mathrm{P} 2 \mathrm{X} 7 \mathrm{R}$ agonists have therapeutic potential for the treatment of skin cancer.

\section{MUSCULOSKELETAL DISEASES}

Reviews that include discussion of the pathophysiology of musculoskeletal diseases have been published (Burnstock et al., 2013; Young et al., 2013; Agrawal and Gartland, 2015; Jørgensen et al., 2015; Orriss et al., 2016). There are multiple purinoceptor subtypes expressed by bone and cartilage, which are potential targets for original therapeutic strategies to inhibit bone resorption in osteoporosis, rheumatoid arthritis (RA), periodontitis and tumour-induced osteolysis.

\section{Muscular Dystrophy}

ATP was used early to treat myopathies, although the mechanism of action was not known. Allopurinol has been used for the treatment of Duchenne muscular dystrophy (DMD). It counteracts low purine nucleotide degradation levels that occur in Duchenne muscle, but chronic administration of allopurinol failed to improve DMD symptoms. Lymphoblastoid cells from DMD patients were found to be highly sensitive to ATP stimulation.

The $m d x$ model of DMD, lacking the dystrophin protein, mimics muscle damage and subsequent regeneration. Sequential expression of $\mathrm{P} 2 \mathrm{X} 5, \mathrm{P}_{2} \mathrm{Y}_{1}$ and $\mathrm{P} 2 \mathrm{X} 2 \mathrm{R}$ was described during muscle regeneration in the $m d x$ model (Ryten et al., 2004). It was claimed recently that a $\mathrm{P}_{2} \mathrm{Y}_{2} \mathrm{R}$ antagonist may ameliorate cardiomyopathy in DMD (De Oliveira Moreira et al., 2017). $\mathrm{P}_{2} \mathrm{Y}_{1} \mathrm{R}$ were expressed on infiltrating immune cells. A purinergic dystrophic phenotype was seen during the earliest stage of developing dystrophic muscle. Dystrophic myoblasts express P2X 4 and P2X7R proteins and it was claimed that antagonists to these receptors may be of potential therapeutic benefit. ATP signalling is altered in muscular dystrophy, but was partly recovered after nifedipine treatment (Valladares et al., 2014). It was claimed that dihydropyridines may be used as a therapeutical tool to reduce muscle damage observed in dystrophic muscles. It was suggested that P2XR antagonists alter the adaptive immune component in the muscle infiltrates in DMD and are a promising therapeutic approach to treat DMD (Gazzerro et al., 2015).

Myofascial pain is a feature of DMD and ATP stimulates myofascial nociceptors. P2X7R upregulation occurs in dystrophic $m d x$ mouse muscles and treatment with $\mathrm{P} 2 \mathrm{X} 7 \mathrm{R}$ antagonists slows the progression of DMD (Sinadinos et al., 2015). Sensitivity to ATP is higher, release of ATP is greater and expression of $\mathrm{P}_{2} \mathrm{Y}_{2} \mathrm{R}$ is increased, but $\mathrm{P} 2 \mathrm{Y}_{1} \mathrm{R}$ expression is decreased in $m d x$ mice. A review about purinergic receptors in DMD was published (Krasowska et al., 2014).

\section{Myasthenia Gravis (MG)}

Myasthenia gravis is an autoimmune disease, which affects the neuromuscular junction. Lack of neuronal $\mathrm{A}_{2 \mathrm{~A}} \mathrm{R}$-mediated $\mathrm{Ca}_{\mathrm{V}}$ 1 (L-type) influx causes tetanic failure in MG (Noronha-Matos et al., 2011). It was suggested that the adenosinergic pathway was dysfunctional in autoimmune $\mathrm{MG}$ and that stimulation of CD73 activity and $\mathrm{A}_{2 \mathrm{~A}} \mathrm{R}$ may have therapeutic potential for $\mathrm{MG}$ (Oliveira et al., 2015).

\section{Fibromyalgia}

Fibromyalgia (a musculoskeletal disease) is characterised by allodynia, as well as mood disorders. In fibromyalgia patients, there are decreased levels of ATP in platelets perhaps contributing to the pathogenesis of the disease (Bazzichi et al., 2008).

\section{Osteoporosis}

Osteoporosis, characterised by bone mass loss (bone mineral density decrease), leads to a high risk of bone fracture. The P2X7R is considered to be important in relation to the treatment of osteoporosis (Kvist et al., 2014). P2X7R plays a critical role in both cortical and cancellous bone mass augmentation and were shown to stimulate cancellous and periosteal bone formation and inhibit cancellous bone resorption during growth. Single nucleotide polymorphisms of the P2X7R gene are associated with the risk of fractures, decrease in bone mineral density and osteoporosis. $\mathrm{P} 2 \mathrm{X} 7 \mathrm{R}$ are involved in the formation of human osteoclasts. P2X7R antagonists are being considered for the treatment of osteoporosis and with remodelling disorders, where bone mass is reduced (Jørgensen et al., 2011). Skeletal pain accompanies osteoporosis and P2X2/3R might have a role in osteoporosis patients under a high bone turnover state (Iba and Yamashita, 2016). P2X5R may be a therapeutic target for treatment for inflammatory bone loss (Kim et al., 2017). Polymorphisms in the P2X4 and P2X7R genes (Husted et al., 2013) and the Leu46Pro polymorphism of the human $\mathrm{P}_{2} \mathrm{Y}_{2} \mathrm{R}$ gene were linked to bone mineral density and the risk of osteoporosis in Dutch fracture patients (Wesselius et al., 2013). It was suggested that clopidogrel, a $\mathrm{P} 2 \mathrm{Y}_{12} \mathrm{R}$ antagonist used for stroke and thrombosis, increases the risk of fractures in osteoporotic patients (Jørgensen et al., 2012). P2 $Y_{13} R$ antagonists have also been proposed for the treatment of osteoporosis (Wang N. et al., 2013).

Adenosine receptors are involved in osteoporosis (McPhee and Whiting, 1989). Trans-differentiation of osteoblasts to adipocytes, which involves $\mathrm{A}_{2 \mathrm{~B}} \mathrm{R}$, may contribute to the pathogenesis of osteoporosis (Rayalam et al., 2011). P1R might be targets for treating osteoporosis and other diseases characterised by excessive bone turnover.

\section{Osteoarthritis (OA)}

Osteoarthritis is a degenerative joint disease as a result of wear of the articular cartilage due to abnormal load to the joint or from infection, trauma or due to ageing. The pain from OA is due largely to inflammation. Increased ATP levels and $5^{\prime}$-nucleotidase activity are present in osteoarthritic joint synovial fluid, compared to the joints from RA patients, particularly from osteoarthritic patients where deposition of 
calcium-containing crystals were also present. ATP contributes to pathologic mineralisation in articular cartilage and therefore P2R antagonists might provide therapeutic tools for crystal-associated arthritis (Costello et al., 2011). ATP levels in knee synovial fluid of patients with $\mathrm{OA}$ are related to pain intensity (Kumahashi et al., 2011). P2X3 and P2X2/3R play an important role in the development of articular hyperalgesia of arthritic joints (Teixeira et al., 2016). In the long-term complications following total hip arthroplasty, different polymorphic variants of the P2X7R are associated with high or reduced periprosthetic osteolysis (Mrazek et al., 2010). There are elevated concentrations of ATP in the synovial fluid of dogs with OA (Torres B.T. et al., 2016). Purinergic signalling, via $\mathrm{P} 2 \mathrm{R}$, produces calcium oscillations in migratory chondrogenic progenitor cells isolated from $\mathrm{OA}$ cartilage (Matta et al., 2015). Platelets promote cartilage repair and chondrocyte proliferation via release of ADP in a rodent model of OA, suggesting that $\mathrm{P}_{2} \mathrm{Y}_{1}$ or $\mathrm{P} 2 \mathrm{Y}_{12} \mathrm{R}$ agonists may be useful for treatment of OA (Zhou Q. et al., 2016). Treatment with the selective P2X7R antagonist, AZD9056, produced painrelieving and anti-inflammatory effects in rats with $\mathrm{OA}(\mathrm{Hu} \mathrm{H}$. et al., 2016).

Adenosine signalling is also involved in OA. Adenosine, produced following breakdown of ATP, is released from chondrocytes and contributes to tissue damage in arthritic conditions. The regulation of LPS-induced IL-6 release involves $\mathrm{A}_{2 \mathrm{~A}} \mathrm{R}$, indicating that adenosine has a regulatory role in controlling osteoclastogenesis and inflammation. Electromagnetic field stimulation up-regulates $A_{2 A} R$ in synovial fibroblasts and adenosine, acting through both $A_{1}$ and $A_{2 A} R$, had anti-inflammatory activity to control joint inflammation (De Mattei et al., 2009). $A_{2 A}$ and $A_{3} R$ agonists modulate prostaglandin $\mathrm{E}_{2}$ and cytokine release in human osteoarthritic fibroblasts (Ongaro et al., 2012). Adenosine receptors also mediate regulation of inflammatory responses in human synoviocytes (Varani et al., 2010b). $\mathrm{A}_{2 \mathrm{~A}} \mathrm{R}$ agonists are used to reduce joint destruction due to septic arthritis (Cohen et al., 2004). $\mathrm{A}_{2 \mathrm{~A}} \mathrm{R}$ deletion resulted in the development of $\mathrm{OA}$ in mice, suggesting that $\mathrm{A}_{2 \mathrm{~A}} \mathrm{R}$ agonists might be a target for the treatment of OA (Corciulo et al., 2017).

\section{Rheumatoid Arthritis (RA)}

The potential involvement in RA of purinergic signalling was recognised first in the 1990's when concentrations of adenosine, after released ATP was broken down, were reduced in synovial fluid in RA compared to OA. Increased levels of adenosine as a treatment for RA was suggested. UTP and ATP activate calcium-mobilising P2UR to synergistically act with IL-1 to stimulate release of prostaglandin $\mathrm{E}_{2}$ from human rheumatoid synovial cells. Hypotonic stress promotes ATP release and cell proliferation via transient receptor potential vanilloid 4 activation in RA rat synovial fibroblasts (Hu et al., 2017).

IL-1 $\beta$ is a proinflammatory cytokine that substantially contributes to the progression of RA. ATP, through P2X7R, induced increased levels of IL-1 $\beta$ in RA patient blood samples compared to control samples. Mononuclear cells from these patients were more sensitive to stimulation by ATP, possibly because of P2X7R genetic polymorphism (Portales-Cervantes et al., 2012). P2X7R are involved in the pathogenesis of RA and systemic lupus erythematosus (Portales-Cervantes et al., 2010). P2X7R mRNA and protein are expressed in human rheumatoid synoviocytes. Studies of arthritis animal models suggest an in vivo role for the P2X7R in the progression of this inflammatory disease. There was a lower incidence and reduced severity of the symptoms of arthritis induced by anti-collagen treatment in P2X7R KO mice. Therefore targeting the P2X7R may be a potential treatment for RA. P2X7R antagonists in the collagen-induced animal model of RA reduced destruction of peripheral inflammatory tissue. In a later study, it was claimed that AZD9056, a P2X7R antagonist, was not effective against RA (Keystone et al., 2012). P2X7R antagonists are being investigated for clinical use against inflammatory joint pain (Beswick et al., 2010). A multicentre, double-blind, placebo-controlled clinical trial showed that ATP infusions reduced inflammation and disease symptoms in patients with RA (Bours et al., 2010).

Bovine chondrocytes express P2X1 and P2X3R and following stimulation there was release of inflammatory mediators. Therefore antagonists to these receptors may be therapeutically useful for articular cartilage resorption and diseases involving inflammation. There was an increased platelet response to ADP in RA patients (Mac Mullan et al., 2010).

Sympathetic nerves mediate proinflammatory responses during the initial phase of arthritis induced by type II collagen, probably via cytokines such as interferon- $\gamma$ released in response to the sympathetic cotransmitters ATP and NA (Straub et al., 2008). $\alpha, \beta$-MeATP-sensitive P2XR (probably P2X3) are expressed on rat knee joint peripheral nociceptive afferent fibres and the increased ATP levels in damaged and inflamed tissues, may contribute to nociception and pain. P2X3R were found on nociceptive sensory fibres in lumber facet joints, where low back pain originates (Ishikawa et al., 2005). Changes in P2X3R expression on DRG neurons that label isolectin B4 were seen following the induction of RA (Averill et al., 2008). P2X3R expressed on trigeminal ganglia also contribute to orofacial pressure pain in monoarthritis of the temporomandibular joint. Plasma extravasation in the rat knee joint induced by bradykinin was enhanced by ATP, released as a cotransmitter from sympathetic nerves. Intravenous guanethidine, which inhibits release of sympathetic cotransmitters, proved to be effective in RA patients. Uncaria tomentosa extract affects the metabolism of adenine nucleotides and has been suggested as an adjuvant to treat arthritis (Castilhos et al., 2015).

The evidence showing a role for adenosine in RA has been reviewed (Varani et al., 2010a). Increased activity of ADA was seen in synovial fluid from patients with RA as well as in rheumatoid synovial fibroblasts (Nakamachi et al., 2003). The ImmuKnow assay might effectively identify RA patients that are more at risk of developing infections (Akimoto et al., 2013). Signalling via the $A_{2 A} R$ caused modification of the cytokine milieu in RA (Masahiro et al., 2003). Over-expression of $A_{3} R$ was observed in peripheral blood mononuclear cells of RA patients. Patients with RA had greater expression $A_{3} R$ in the synovium (Stamp et al., 2012). There are increased levels of $A_{2 A}$ and $A_{3} R$ on the lymphocytes and neutrophils of RA patients. In the dorsal horn of rats with induced $\mathrm{RA}, \mathrm{A}_{1} \mathrm{R}$ agonists decreased 
activation of $c$-fos and astrocytes. In a later study of the adjuvantinduced monoarthritis model an $\mathrm{A}_{3}$ specific agonist prevented bone resorption. ATP, working via $\mathrm{A}_{2} \mathrm{R}$, also reduced joint injury. A phase II clinical trial provided evidence for $\mathrm{A}_{3} \mathrm{R}$ agonists as a treatment for RA (Fishman et al., 2008; Silverman et al., 2008). CGS 21680, an agonist of the $A_{2 A} R$, reduced progression of murine type II collagen-induced arthritis (Mazzon et al., 2011). $\mathrm{A}_{2 \mathrm{~A}} \mathrm{R}$ agonists ameliorate adjuvant-induced arthritis in rats (Vincenzi et al., 2013). Methotrexate (MTX) is often used to treat RA. In human joints with inflammatory disease, MTX treatment involved $\mathrm{A}_{2 \mathrm{~A}} \mathrm{R}$. Adenosine via $\mathrm{A}_{2 \mathrm{~B}} \mathrm{R}$ prevented MTX-induced inhibition of osteoclast bone destruction in arthritis induced by adjuvant (Teramachi et al., 2011). In RA patients treated with MTX, studies of polymorphisms of the genes involved in adenosine release concluded that genotyping may help identify patients who would most benefit from MTX treatment (Wessels et al., 2006). Anti-tumour necrosis factor- $\alpha$ has also been used to treat RA, but it raises the risk of reactivating tuberculosis. ADA assay is a specific and sensitive test for the quick diagnosis of rheumatoid effusions (Zakeri et al., 2012).

\section{Tooth Pain}

There are many sensory nerves, originating in the trigeminal ganglia, expressing $\mathrm{P} 2 \mathrm{X} 3 \mathrm{R}$ in tooth pulp and ATP is released from odontoblasts in response to mechanical stimulation to act on these receptors, resulting in pain (Shibukawa et al., 2015). LPS-induced pulp inflammation increased the expression of P2XR in trigeminal sensory nerves (Chen et al., 2014). Mechanical or cold stimulation of odontoblast processes in dentin tubules, results in ATP release and dental pain (Liu X. et al., 2015). Therefore P2X3R antagonists may be therapeutically useful to reduce toothache.

\section{Bone Cancer Pain}

Bone metastases, common in prostate and breast cancer patients, may cause substantial bone loss and pain. Purinergic signalling involvement in bone cancer was initially reported in the 1990's when P2U (i.e., $\mathrm{P}_{2} \mathrm{Y}_{2} / \mathrm{P}_{2} \mathrm{Y}_{4}$ ) receptors were cloned from osteoclastoma. The expression of $\mathrm{P} 2 \mathrm{Y}_{2} \mathrm{R}$ from human osteoclasts from bone giant cell tumour was later reported. Butyl benzyl phthalate, which interferes with mammalian ion channel receptors, inhibited ATP-induced cell proliferation via P2XR in human osteosarcoma HOS cells (Liu and Chen, 2010). ATP was used in autologous bone marrow transplantation for removing residual tumour cells. In mice, a type of human apyrase, APT102, in addition to aspirin disrupts bone metastasis (Uluçkan et al., 2008). Release of ATP from tumour cells further stimulates osteoclast formation and activity, contributing to bone destruction that often happens around tumour metastases. Bisphosphonates, used to treat osteoporosis, treat bone cancer and may involve apoptosis induced by ApppI, an ATP analogue produced by bisphosphonates (Sillero et al., 2009).

Purinergic signalling plays a role in bone cancer pain. Bone pain can be relieved by radiation therapy, which may be related to the $\mathrm{Ca}^{2+}$-signalling cascade, mediated by P2X6R. In a mouse model of cancer pain, increased expression of P2X3R on calcitonin gene-related peptide immunoreactive DRG neurons during tumour growth, it was claimed that ATP had a role in cancer-related pain (Liu M. et al., 2013). In rats, systemic inhibition of $\mathrm{P} 2 \mathrm{X} 3$ and $\mathrm{P} 2 \mathrm{X} 2 / 3 \mathrm{R}$ with AF-353 strongly attenuated bone cancer pain-related behaviour (Kaan et al., 2010). In mice, administration of A-317491, a selective P2X3 and P2X2/3R antagonist, attenuated the early stages of bone pain in cancer (Hansen et al., 2012). Functional up-regulation of P2X3R has been described in DRG of bone cancer pain in a rat model (Wu et al., 2012).

In P2X7R KO mice, bone cancer pain-related behaviours had an earlier onset (Hansen et al., 2011). Most human osteosarcomas expressed P2X7R isoforms A and B (Giuliani et al., 2014) and $\mathrm{P} 2 \mathrm{X} 7 \mathrm{R}$ are involved in cancer-induced bone pain and P2X7R antagonists were suggested as a useful analgesic target (Falk et al., 2015). $P 2 Y_{1} R$ signalling in the DRG and spinal cord may mediate pain from bone cancer (Chen et al., 2012). Activation of $K_{A T P}$ channels at the spinal cord level reduces pain associated with bone cancer (Xia et al., 2014). Stimulation by AMP-activated protein kinase suppresses neuroinflammation and reduces bone cancer pain (Song et al., 2015).

\section{Myeloma}

Multiple myeloma (cancer of plasma cells) involves osteolytic bone lesions, due largely to enhanced osteoclast activity. $\mathrm{A}_{2} \mathrm{R}$ may be therapeutically useful to treat and prevent multiple myeloma-induced bone disease as activation of $A_{2 A} R$ reduces osteoclast function, while activation of $\mathrm{A}_{2 \mathrm{~B}} \mathrm{R}$ stimulates osteoblast differentiation (He et al., 2012). 8-Amino-adenosine is another possible therapeutic compound for the treatment of multiple myeloma.

\section{Severe Combined Immunodeficiency}

A major cause of severe combined immunodeficiency are genetic defects in the ADA gene. Lack of ADA causes accumulation of adenosine. Bone defects as a result of reduced osteoclastogenesis together with a defect in osteoblast function leading to low bone formation were seen in about a half of early-onset ADA-deficient patients (Sauer et al., 2009). Further, the microenvironment of bone marrow in ADA KO mice had a lower ability to support haematopoiesis. In $\mathrm{ADA} \mathrm{KO}$ mice, treatment with gene therapy, bone marrow transplantation or enzyme replacement, led to a full recovery. ADA-transduced hematopoietic stem cell gene therapy also enhanced the growth of children with this disease.

\section{Dwarfism (Achondroplasia)}

This is a congenital dysplasia of the skeleton as a result in a mutation in the gene encoding fibroblast growth factor receptor type 3 (FGR3). $\mathrm{Ap}_{4} \mathrm{~A}$ diminished the expression of the achondroplasic FGFR3 receptor and $\mathrm{P} 2 \mathrm{Y}_{1}, \mathrm{P}_{2} \mathrm{Y}_{2}, \mathrm{P} 2 \mathrm{Y}_{6}$, and $\mathrm{P} 2 \mathrm{Y}_{11} \mathrm{R}$ are expressed by achondroplasic chondrocytes mediating the action of $\mathrm{Ap}_{4} \mathrm{~A}$ (Guzmán-Aránguez et al., 2008). $\mathrm{Ap}_{4} \mathrm{~A}$ reversed the morphological changes supporting a therapeutic role for $\mathrm{Ap}_{4} \mathrm{~A}$ as a possible treatment of dwarfism (Huete et al., 2011). 


\section{Paget's Disease}

There is an increase in osteoclast numbers in Paget's disease, leading to an increase in bone resorption and a high turnover of bone. Bisphosphonates have been employed as a treatment for Paget's disease and P2X7 antagonists have also been considered (Agrawal et al., 2010).

\section{Ossification of the Posterior Longitudinal Ligament of the Spine}

This disease causes neurological damage as a result of ectopic bone formation in spinal ligaments. In this disease, extracellular ATP in ossification of cell cultures of the posterior longitudinal ligament of the spine (OPLL) is increased. $\mathrm{P}_{2} \mathrm{Y}_{1} \mathrm{R}$ are highly expressed in OPLL cells. Mechanical stress and ATP increase the levels of osteopontin and alkaline phosphatase mRNA in OPLL cells, effects that can be inhibited by MRS2179, a selective $\mathrm{P}_{2} \mathrm{Y}_{1} \mathrm{R}$ antagonist. Over-expression of $\mathrm{P} 2 \mathrm{Y}_{1} \mathrm{R}$ in OPLL-induced mineralisation resulted in ectopic bone formation in the spinal ligament cells of patients with OPLL (Tanaka et al., 2011).

\section{CONCLUDING COMMENTS}

Clinical interventions involving purinergic signalling are just beginning. However, the beginning and future of purinergic compounds for the treatment of a wide range of diseases is described in this review. $\mathrm{P} 2 \mathrm{Y}_{12} \mathrm{R}$ antagonists, such as clopidogrel and ticagrelor, are currently in wide use for stroke and thrombosis, as are $\mathrm{P} 2 \mathrm{Y}_{2} \mathrm{R}$ agonists for dry eye and $\mathrm{A}_{1} \mathrm{R}$ agonists for supraventricular tachycardia. The use of P2X7R antagonists for the treatment of inflammatory diseases is promising, but the presence of polymorphic variations of this receptor is holding up the development of selective antagonists appropriate for each disease. P2X3R antagonists are in clinical trials for use against visceral pain, chronic cough and hypertension. $\mathrm{A}_{2 \mathrm{~A}} \mathrm{R}$ agonists are in use for the treatment of $\mathrm{PD}$, and perhaps soon in wider use. P2X1R antagonists are being investigated for treatment of bladder disorders and hypertension, while P2X4, P2X7 and $\mathrm{A}_{3} \mathrm{R}$ antagonists are being explored for neuropathic pain. Treatments with inhalation of ectonucleotidases to alter the balance of ATP and adenosine and inhibitors of ATP release, are also a therapeutic approach being explored. The development of novel purinergic compounds by medicinal chemists that are orally available and stable in vivo would be a significant advantage in developing therapeutic approaches, including centrally penetrant P2X7R antagonists (Able et al., 2011).

The majority of the therapeutic approaches for many heart disorders based on purinergic signalling manipulation are not fully understood yet and strategies to overcome the side-effects

\section{REFERENCES}

Abdulqawi, R., Dockry, R., Holt, K., Layton, G., Mccarthy, B. G., Ford, A. P., et al. (2015). P2X3 receptor antagonist (AF-219) in refractory chronic cough: a randomised, double-blind, placebo-controlled phase 2 study. Lancet 385, 1198-1205. doi: 10.1016/S0140-6736(14)61255-1 of treatment need to be considered. The pathophysiological roles of purinergic signalling in blood vessels are clearer and it plays an important role in controlling vascular tone and remodelling. Immunologic factors related to purinergic signalling are attracting more attention and should be considered (Cekic and Linden, 2016). Human embryonic stem cells are able to self-renew and have the potential to differentiate into different cell types, including cardiovascular progenitor cells. This system of differentiation is being investigated for cardiac regenerative therapy (Huang et al., 2016). The single nucleotide polymorphisms in purinergic receptor genes and their association with diseases are being explored for potential use as diagnosis biomarkers (see Caseley et al., 2014). Purinoceptors modulate neural stem cell proliferation, differentiation, migration and cell death and could be therapeutic approaches for the treatment of neurological and psychiatric illnesses (Illes and Rubini, 2017). MicroRNAs modulating purinergic signalling are gaining interest as potential original therapeutic targets and disease biomarkers (Ferrari et al., 2016a).

Although still in its infancy, clinical use of purinergic compounds has started. Several relevant pharmacological interventions are currently in clinical use. The lack of more established purinergic therapies may be due to there being relatively few receptor subtype-specific agonists and antagonists that are both effective and stable in vivo (see Jacobson and Muller, 2016). In some situations, a degree of redundancy is present, with several different subtypes of receptor mediating similar functional effects. Purinergic signalling is implicated in multiple disorders and therefore offers many potential future therapeutic targets. It should be noted, however, that since most purinoceptors are ubiquitous, to selectively target specific cell types may prove to be a challenge. As well as the development of selective agonists and antagonists, therapeutic strategies will probably include compounds that control P2R expression, inhibitors of extracellular ATP breakdown and inhibitors or enhancers of ATP transport. Understanding the interactions of purinergic signalling with other established signalling systems will be necessary.

\section{AUTHOR CONTRIBUTIONS}

The author confirms being the sole contributor of this work and approved it for publication.

\section{ACKNOWLEDGMENT}

I am greatly indebted to Dr. Gillian E. Knight for the excellent editorial work in the preparation of this manuscript.

Able, S. L., Fish, R. L., Bye, H., Booth, L., Logan, Y. R., Nathaniel, C., et al. (2011). Receptor localization, native tissue binding and ex vivo occupancy for centrally penetrant P2X7 antagonists in the rat. Br. J. Pharmacol. 162, 405-414. doi: 10.1111/j.1476-5381.2010.01025.x

Abraham, M. K., Nolte, A., Reus, R., Behring, A., Zengerle, D., AvciAdali, M., et al. (2015). In vitro study of a novel stent coating using 
modified CD39 messenger RNA to potentially reduce stent angioplastyassociated complications. PLOS ONE 10:e0138375. doi: 10.1371/journal.pone. 0138375

Acevedo, C. G., Huambachano, A., Perez, E., Rojas, S., Bravo, I., and Contreras, E. (1997). Effect of ethanol on human placental transport and metabolism of adenosine. Placenta 18, 387-392. doi: 10.1016/S0143-4004(97)80038-0

Adinolfi, E., Capece, M., Franceschini, A., Falzoni, S., Giuliani, A. L., Rotondo, A., et al. (2015). Accelerated tumor progression in mice lacking the ATP receptor P2X7. Cancer Res. 75, 635-644. doi: 10.1158/0008-5472.CAN-14- 1259

Adinolfi, E., Raffaghello, L., Giuliani, A. L., Cavazzini, L., Capece, M., Chiozzi, P., et al. (2012). Expression of P2X7 receptor increases in vivo tumor growth. Cancer Res. 72, 2957-2969. doi: 10.1158/0008-5472.CAN-11-1947

Agarwal, R., and Agarwal, P. (2014). Newer targets for modulation of intraocular pressure: focus on adenosine receptor signaling pathways. Expert Opin. Ther. Targets 18, 527-539. doi: 10.1517/14728222.2014.888416

Aghaei, M., Karami-Tehrani, F., Panjehpour, M., Salami, S., and Fallahian, F. (2012). Adenosine induces cell-cycle arrest and apoptosis in androgendependent and -independent prostate cancer cell lines, LNcap-FGC-10, DU-145, and PC3. Prostate 72, 361-375. doi: 10.1002/pros.21438

Agrawal, A., Buckley, K. A., Bowers, K., Furber, M., Gallagher, J. A., and Gartland, A. (2010). The effects of P2X7 receptor antagonists on the formation and function of human osteoclasts in vitro. Purinergic Signal. 6, 307-315. doi: $10.1007 /$ s11302-010-9181-z

Agrawal, A., and Gartland, A. (2015). P2X7 receptors: role in bone cell formation and function. J. Mol. Endocrinol. 54, R75-R88. doi: 10.1530/JME-14-0226

Agteresch, H. J., Burgers, S. A., Van Der Gaast, A., Wilson, J. H., and Dagnelie, P. C. (2003). Randomized clinical trial of adenosine $5^{\prime}$-triphosphate on tumor growth and survival in advanced lung cancer patients. Anticancer Drugs 14, 639-644. doi: 10.1097/00001813-200309000-00009

Aguiari, G., Varani, K., Bogo, M., Mangolini, A., Vincenzi, F., Durante, C., et al. (2009). Deficiency of polycystic kidney disease-1 gene (PKD1) expression increases A3 adenosine receptors in human renal cells: implications for cAMPdependent signalling and proliferation of PKD1-mutated cystic cells. Biochim. Biophys. Acta 1792, 531-540. doi: 10.1016/j.bbadis.2009.03.001

Ahsan, M. K. (2011). The adenosine A2 receptor enhances primary rat HSC proliferation and inhibits senescence by down-regulation of P53 and RB. Hepatology 54, 750A-751A. doi: 10.3389/fphar.2014.00069

Aikin, R. A. (2013). Targeting purinergic receptors in islet transplantation. Diabetes Metab. Res. Rev. 62, 1394-1395. doi: 10.2337/db13-0211

Akimoto, M., Yunoue, S., Otsubo, H., Yoshitama, T., Kodama, K., Matsushita, K., et al. (2013). Assessment of peripheral blood CD4+ adenosine triphosphate activity in patients with rheumatoid arthritis. Mod. Rheumatol. 23, 19-27. doi: 10.1007/s10165-012-0621-1

Akino, H., Nagase, K., Watanabe, N., Tanase, K., Oyama, N., Miwa, Y., et al. (2011). ATP release from blassers is increased in-vivo and suppressed by alpha1 adrenoceptor blocker in a rat model of bladder outlet obstruction. Eur. Urol. Suppl. 10, 303-304. doi: 10.1016/S1569-9056(11)60955-X

Albert, U., De Cori, D., Aguglia, A., Barbaro, F., Bogetto, F., and Maina, G. (2015). Increased uric acid levels in bipolar disorder subjects during different phases of illness. J. Affect. Disord 173, 170-175. doi: 10.1016/j.jad.2014.11.005

Alberto, A. V., Faria, R. X., De Menezes, J. R., Surrage, A., Da Rocha, N. C., Ferreira, L. G., et al. (2016). Role of P2 receptors as modulators of rat eosinophil recruitment in allergic inflammation. PLOS ONE 11:e0145392. doi: 10.1371/ journal.pone. 0145392

Alexopoulos, D., Xanthopoulou, I., Moulias, A., and Lekakis, J. (2016). Long-term P2Y12-receptor antagonists in post-myocardial infarction patients: facing a new trilemma? J. Am. Coll. Cardiol. 68, 1223-1232. doi: 10.1016/j.jacc.2016.05.088

Almeida-da-Silva, C. L., Morandini, A. C., Ulrich, H., Ojcius, D. M., and CoutinhoSilva, R. (2016). Purinergic signaling during Porphyromonas gingivalis infection. Biomed. J. 39, 251-260. doi: 10.1016/j.bj.2016.08.003

Alves, L. A., Bezerra, R. J., Faria, R. X., Ferreira, L. G., and Da Silva Frutuoso, V. (2013). Physiological roles and potential therapeutic applications of the P2X7 receptor in inflammation and pain. Molecules 18, 10953-10972. doi: 10.3390/ molecules 180910953

Amaral, E. P., Ribeiro, S. C., Lanes, V. R., Almeida, F. M., De Andrade, M. R., Bomfim, C. C., et al. (2014). Pulmonary infection with hypervirulent Mycobacteria reveals a crucial role for the P2X7 receptor in aggressive forms of tuberculosis. PLOS Pathog. 10:e1004188. doi: 10.1371/journal.ppat.1004188
Amaral, S. S., Oliveira, A. G., Marques, P. E., Quintão, J. L., Pires, D. A., Resende, R. R., et al. (2013). Altered responsiveness to extracellular ATP enhances acetaminophen hepatotoxicity. Cell Commun. Signal. 11:10. doi: 10.1186/1478811X-11-10

Amoroso, F., Capece, M., Rotondo, A., Cangelosi, D., Ferracin, M., Franceschini, A., et al. (2015). The P2X7 receptor is a key modulator of the PI3K/GSK3 $\beta /$ VEGF signaling network: evidence in experimental neuroblastoma. Oncogene 34, 5240-5251. doi: 10.1038/onc.2014.444

Andersson, K. E. (2001). Neurotransmission and drug effects in urethral smooth muscle. Scand. J. Urol. Nephrol. Suppl. 207, 26-34. doi: 10.1080/ 003655901750174854

Andersson, K. E., Chapple, C. R., and Höfner, K. (2002). Future drugs for the treatment of benign prostatic hyperplasia. World J. Urol. 19, 436-442. doi: 10.1007/s00345-002-0253-8

Andersson, K. E., and Hedlund, P. (2002). Pharmacologic perspective on the physiology of the lower urinary tract. Urology 60, 13-20. doi: 10.1016/S00904295(02)01786-7

Andrés, R. M., Terencio, M. C., Arasa, J., Payá, M., Valcuende-Cavero, F., Navalón, P., et al. (2017). Adenosine A2A and A2B receptors differentially modulate keratinocyte proliferation: possible deregulation in psoriatic epidermis. J. Invest. Dermatol. 137, 123-131. doi: 10.1016/j.jid.2016. 07.028

Antonioli, L., Blandizzi, C., Csóka, B., Pacher, P., and Haskó, G. (2015). Adenosine signalling in diabetes mellitus - pathophysiology and therapeutic considerations. Nat. Rev. Endocrinol. 11, 228-241. doi: 10.1038/nrendo.2015.10

Antonioli, L., Colucci, R., Pellegrini, C., Giustarini, G., Tuccori, M., Blandizzi, C., et al. (2013). The role of purinergic pathways in the pathophysiology of gut diseases: pharmacological modulation and potential therapeutic applications. Pharmacol. Ther. 139, 157-188. doi: 10.1016/j.pharmthera.2013.04.002

Antonioli, L., Csóka, B., Fornai, M., Colucci, R., Kókai, E., Blandizzi, C., et al. (2014a). Adenosine and inflammation: what's new on the horizon? Drug Discov. Today 19, 1051-1068. doi: 10.1016/j.drudis.2014.02.010

Antonioli, L., Giron, M. C., Colucci, R., Pellegrini, C., Sacco, D., Caputi, V., et al. (2014b). Involvement of the P2X7 purinergic receptor in colonic motor dysfunction associated with bowel inflammation in rats. PLOS ONE 9:e116253. doi: 10.1371/journal.pone.0116253

Apolloni, S., Amadio, S., Parisi, C., Matteucci, A., Potenza, R. L., Armida, M., et al. (2014). Spinal cord pathology is ameliorated by P2X7 antagonism in a SOD1-mutant mouse model of amyotrophic lateral sclerosis. Dis. Model Mech. 7, 1101-1109. doi: 10.1242/dmm.017038

Apostolidis, A., Popat, R., Yiangou, Y., Cockayne, D., Ford, A. P., Davis, J. B., et al. (2005). Decreased sensory receptors P2X3 and TRPV1 in suburothelial nerve fibers following intradetrusor injections of botulinum toxin for human detrusor overactivity. J. Urol. 174, 977-982. doi: 10.1097/01.ju.0000169481.42259.54

Aprile-Garcia, F., Metzger, M. W., Paez-Pereda, M., Stadler, H., Acuña, M., Liberman, A. C., et al. (2016). Co-Expression of wild-type P2X7R with Gln460Arg variant alters receptor function. PLOS ONE 11:e0151862. doi: 10.1371/journal.pone.0151862

Aronsson, P., Johnsson, M., Vesela, R., Winder, M., and Tobin, G. (2012). Adenosine receptor antagonism suppresses functional and histological inflammatory changes in the rat urinary bladder. Auton. Neurosci. 171, 49-57. doi: 10.1016/j.autneu.2012.10.006

Arulkumaran, N., Unwin, R. J., and Tam, F. W. (2011). A potential therapeutic role for P2X7 receptor (P2X7R) antagonists in the treatment of inflammatory diseases. Expert Opin. Investig. Drugs 20, 897-915. doi: 10.1517/13543784.2011. 578068

Ashimi, O., Parchim, N., Zhang, Y., Song, A., Iriyama, T., Sibai, B., et al. (2015). Circulating adenosine levels are elevated in pregnant women with sickle cell disease through 2,3-DPG induction. Am. J. Obstet. Gynecol. 212, S409-S410. doi: 10.1016/j.ajog.2014.10.1060

Atiemo, H., Wynes, J., Chuo, J., Nipkow, L., Sklar, G. N., and Chai, T. C. (2005). Effect of botulinum toxin on detrusor overactivity induced by intravesical adenosine triphosphate and capsaicin in a rat model. Urology 65, 622-626. doi: 10.1016/j.urology.2004.10.057

Avendano, B. C., Montero, T. D., Chávez, C. E., Von Bernhardi, R., and Orellana, J. A. (2015). Prenatal exposure to inflammatory conditions increases Cx43 and Panx1 unopposed channel opening and activation of astrocytes in the offspring effect on neuronal survival. Glia 63, 2058-2072. doi: 10.1002/glia.22877 
Averill, S., Inglis, J. J., King, V. R., Thompson, S. W., Cafferty, W. B., Shortland, P. J., et al. (2008). Reg-2 expression in dorsal root ganglion neurons after adjuvant-induced monoarthritis. Neuroscience 155, 1227-1236. doi: 10.1016/j. neuroscience.2008.06.049

Ayata, C. K., Ganal, S. C., Hockenjos, B., Willim, K., Vieira, R. P., Grimm, M., et al. (2012). Purinergic P2Y2 receptors promote neutrophil infiltration and hepatocyte death in mice with acute liver injury. Gastroenterology 143, 1620-1629. doi: 10.1053/j.gastro.2012.08.049

Azimi, I., Beilby, H., Davis, F. M., Marcial, D. L., Kenny, P. A., Thompson, E. W., et al. (2016). Altered purinergic receptor-Ca2+ signaling associated with hypoxia-induced epithelial-mesenchymal transition in breast cancer cells. Mol. Oncol. 10, 166-178. doi: 10.1016/j.molonc.2015.09.006

Bai, H. Y., and Li, A. P. (2013). P2X7 receptors in cerebral ischemia. Neurosci. Bull. 29, 390-398. doi: 10.1007/s12264-013-1338-7

Baranowska-Bosiacka, I., Listos, J., Gutowska, I., Hoy-Mokrzynska, A., KolasaWolosiuk, A., Tarnowski, M., et al. (2016). Effects of perinatal exposure to lead $(\mathrm{Pb})$ on purine receptor expression in the brain and gliosis in rats tolerant to morphine analgesia. Toxicology 339, 19-33. doi: 10.1016/j.tox.2015.10.003

Barron, M. L., Werry, E. L., Mcgregor, I. S., and Kassiou, M. (2014). "P2X7 in bipolar and depressive disorders," in Pathologies of Calcium Channels, eds N. Weiss and A. Koschak (Berlin: Springer), 635-661.

Bartlett, R., Stokes, L., and Sluyter, R. (2014). The P2X7 receptor channel: recent developments and the use of $\mathrm{P} 2 \mathrm{X} 7$ antagonists in models of disease. Pharmacol. Rev. 66, 638-675. doi: 10.1124/pr.113.008003

Bartoli, F., Carrà, G., and Clerici, M. (2017a). Update on bipolar disorder biomarker candidates: what about uric acid/adenosine hypothesis? Expert Rev. Mol. Diagn. 17, 105-106. doi: 10.1080/14737159.2017.1270757

Bartoli, F., Crocamo, C., Dakanalis, A., Brosio, E., Miotto, A., Capuzzi, E., et al. (2017b). Purinergic system dysfunctions in subjects with bipolar disorder: a comparative cross-sectional study. Compr. Psychiatry 73, 1-6. doi: 10.1016/j. comppsych.2016.09.011

Bar-Yehuda, S., Stemmer, S. M., Madi, L., Castel, D., Ochaion, A., Cohen, S., et al. (2008). The A3 adenosine receptor agonist CF102 induces apoptosis of hepatocellular carcinoma via de-regulation of the Wnt and NF- $\mathrm{kB}$ signal transduction pathways. Int. J. Oncol. 33, 287-295.

Basoglu, O. K., Barnes, P. J., Kharitonov, S. A., and Pelleg, A. (2015). Effects of aerosolized adenosine $5^{\prime}$-triphosphate in smokers and patients with COPD. Chest 148, 430-435. doi: 10.1378/chest.14-2285

Basoglu, O. K., Pelleg, A., Kharitonov, S. A., and Barnes, P. J. (2017). Contrasting effects of ATP and adenosine on capsaicin challenge in asthmatic patients. Pulm. Pharmacol. Ther. 45, 13-18. doi: 10.1016/j.pupt.2017.04.004

Basu, S., Barawkar, D. A., Ramdas, V., Patel, M., Waman, Y., Panmand, A., et al. (2017). Design and synthesis of novel xanthine derivatives as potent and selective $\mathrm{A} 2 \mathrm{~B}$ adenosine receptor antagonists for the treatment of chronic inflammatory airway diseases. Eur. J. Med. Chem. 134, 218-229. doi: 10.1016/j. ejmech.2017.04.014

Baudelet, D., Lipka, E., Millet, R., and Ghinet, A. (2015). Involvement of the P2X7 purinergic receptor in inflammation: an update of antagonists series since 2009 and their promising therapeutic potential. Curr. Med. Chem. 22, 713-729. doi: 10.2174/0929867322666141212120926

Bazzichi, L., Giannaccini, G., Betti, L., Fabbrini, L., Schmid, L., Palego, L., et al. (2008). ATP, calcium and magnesium levels in platelets of patients with primary fibromyalgia. Clin. Biochem. 41, 1084-1090. doi: 10.1016/j.clinbiochem.2008. 06.012

Beamer, E., Fischer, W., and Engel, T. (2017). The ATP-gated P2X7 receptor as a target for the treatment of drug-resistant epilepsy. Front. Neurosci. 11:21. doi: 10.3389/fnins.2017.00021

Beamer, E., Gölöncsér, F., Horváth, G., Bekö, K., Otrokocsi, L., Koványi, B., et al. (2016). Purinergic mechanisms in neuroinflammation: an update from molecules to behavior. Neuropharmacology 104, 94-104. doi: 10.1016/j. neuropharm.2015.09.019

Beaucage, K. L., Xiao, A., Pollmann, S. I., Grol, M. W., Beach, R. J., Holdsworth, D. W., et al. (2014). Loss of P2X7 nucleotide receptor function leads to abnormal fat distribution in mice. Purinergic Signal. 10, 291-304. doi: 10.1007/s11302013-9388-x

Beckel, J. M., Lu, W., Civan, M. M., and Mitchell, C. H. (2016). Treatment of retinal disorders with purinergic drugs: beyond receptors. J. Ocul. Pharmacol. Ther. 32, 488-489. doi: 10.1089/jop.2016.29020.jbe
Beckenkamp, A., Santana, D. B., Bruno, A. N., Calil, L. N., Casali, E. A., Paccez, J. D., et al. (2014). Ectonucleotidase expression profile and activity in human cervical cancer cell lines. Biochem. Cell Biol. 92, 95-104. doi: 10.1139/bcb-20130051

Belhassen, B., and Michowitz, Y. (2016). Unmasking right atrial fibrillation: a new indication of adenosine triphosphate test? Heart Rhythm 13, 364-365. doi: 10.1016/j.hrthm.2015.10.020

Ben-Ari, Z., Pappo, O., Yitzhaki, S., Cheporko, Y., Shainberg, A., Zinman, T., et al. (2009). Uridine- $5^{\prime}$-triphosphate protects against hepaticischemic/reperfusion injury in mice. Transplantation 87, 1155-1162. doi: 10.1097/TP.0b013e31819e3cdc

Benitez-Rajal, J., Lorite, M. J., Burt, A. D., Day, C. P., and Thompson, M. G. (2006). Phospholipase D and extracellular signal-regulated kinase in hepatic stellate cells: effects of platelet-derived growth factor and extracellular nucleotides. Am. J Physiol. Gastrointest. Liver Physiol. 291, G977-G986. doi: 10.1152/ajpgi.00041. 2006

Bergamin, L. S., Braganhol, E., Figueiró, F., Casali, E. A., Zanin, R. F., Sévigny, J., et al. (2015). Involvement of purinergic system in the release of cytokines by macrophages exposed to glioma-conditioned medium. J. Cell Biochem. 116, 721-729. doi: $10.1002 / j \mathrm{cb} .25018$

Besnard, A., Gautherot, J., Julien, B., Tebbi, A., Garcin, I., Doignon, I., et al. (2016). The P2X4 purinergic receptor impacts liver regeneration after partial hepatectomy in mice through the regulation of biliary homeostasis. Hepatology 64, 941-953. doi: 10.1002/hep.28675

Beswick, P. J., Billinton, A., Chambers, L. J., Dean, D. K., Fonfria, E., Gleave, R. J., et al. (2010). Structure-activity relationships and in vivo activity of (1H-pyrazol4-yl)acetamide antagonists of the P2X7 receptor. Bioorg. Med. Chem. Lett. 20, 4653-4656. doi: 10.1016/j.bmcl.2010.05.107

Bhattacharya, A., and Biber, K. (2016). The microglial ATP-gated ion channel P2X7 as a CNS drug target. Glia 64, 1772-1787. doi: 10.1002/glia. 23001

Bhave, S., Gade, A., Kang, M., Hauser, K. F., Dewey, W. L., and Akbarali, H. I. (2017). Connexin-purinergic signaling in enteric glia mediates the prolonged effect of morphine on constipation. FASEB J. 31, 2649-2660. doi: 10.1096/fj. 201601068R

Boldrini, L., Giordano, M., Alì, G., Melfi, F., Romano, G., Lucchi, M., et al. (2015). P2X7 mRNA expression in non-small cell lung cancer: MicroRNA regulation and prognostic value. Oncol. Lett. 9, 449-453.

Borea, P. A., Gessi, S., Merighi, S., and Varani, K. (2016). Adenosine as a multisignalling guardian angel in human diseases: when, where and how does it exert its protective effects? Trends Pharmacol. Sci. 37, 419-434. doi: 10.1016/j.tips. 2016.02.006

Borea, P. A., Varani, K., Vincenzi, F., Baraldi, P. G., Tabrizi, M. A., Merighi, S., et al. (2015). The A3 adenosine receptor: history and perspectives. Pharmacol. Rev. 67, 74-102. doi: 10.1124/pr.113.008540

Borel, F., Han, R., Visser, A., Petry, H., Van Deventer, S. J., Jansen, P. L., et al. (2012). Adenosine triphosphate-binding cassette transporter genes up-regulation in untreated hepatocellular carcinoma is mediated by cellular microRNAs. Hepatology 55, 821-832. doi: 10.1002/hep. 24682

Boros, D., Thompson, J., and Larson, D. F. (2016). Adenosine regulation of the immune response initiated by ischemia reperfusion injury. Perfusion 31, 103-110. doi: 10.1177/0267659115586579

Bou Ghanem, E. N., Clark, S., Roggensack, S. E., Mciver, S. R., Alcaide, P., Haydon, P. G., et al. (2015). Extracellular adenosine protects against Streptococcus pneumoniae lung infection by regulating pulmonary neutrophil recruitment. PLOS Pathog. 11:e1005126. doi: 10.1371/journal.ppat.1005126

Bours, M. J., Peeters, R. H., Landewé, R. B., Beijer, S., Arts, I. C., and Dagnelie, P. C. (2010). Adenosine $5^{\prime}$-triphosphate infusions reduced disease activity and inflammation in a patient with active rheumatoid arthritis. Rheumatology (Oxford) 49, 2223-2225. doi: 10.1093/rheumatology/keq177

Bowser, J. L., Blackburn, M. R., Shipley, G. L., Molina, J. G., Dunner, K. Jr., and Broaddus, R. R. (2016). Loss of CD73-mediated actin polymerization promotes endometrial tumor progression. J. Clin. Invest. 126, 220-238. doi: 10.1172/ JCI79380

Boy, C., Meyer, P. T., Kircheis, G., Holschbach, M. H., Herzog, H., Elmenhorst, D., et al. (2008). Cerebral A1 adenosine receptors (A1AR) in liver cirrhosis. Eur. J. Nucl. Med. Mol. Imaging 35, 589-597. doi: 10.1007/s00259-007-0586-z 
Braganhol, E., Kukulski, F., Lévesque, S. A., Fausther, M., Lavoie, E. G., ZanottoFilho, A., et al. (2015). Nucleotide receptors control IL-8/CXCL8 and MCP1/CCL2 secretions as well as proliferation in human glioma cells. Biochim. Biophys. Acta 1852, 120-130. doi: 10.1016/j.bbadis.2014.10.014

Bravo, D., Maturana, C. J., Pelissier, T., Hernández, A., and Constandil, L. (2015). Interactions of pannexin 1 with NMDA and P2X7 receptors in central nervous system pathologies: possible role on chronic pain. Pharmacol. Res. 101, 86-93. doi: 10.1016/j.phrs.2015.07.016

Brock, J. A., and Van Helden, D. F. (1995). Enhanced excitatory junction potentials in mesenteric arteries from spontaneously hypertensive rats. Pflügers Arch. Eur. J. Physiol. 430, 901-908. doi: 10.1007/BF01837403

Broderick, P. A., and Malave, L. B. (2014). Cocaine shifts the estrus cycle out of phase and caffeine restores it. J. Caffeine Res. 4, 109-113. doi: 10.1089/jcr.2014. 0015

Brouns, I., Van Genechten, J., Burnstock, G., Timmermans, J.-P., and Adriaensen, D. (2003). Ontogenesis of P2X3 receptor-expressing nerve fibres in the rat lung, with special reference to neuroepithelial bodies. Biomed. Res. 14, 80-86.

Bukhari, M., Deng, H., Jones, N., Towne, Z., Woodworth, C. D., and Samways, D. S. (2015). Selective permeabilization of cervical cancer cells to an ionic DNAbinding cytotoxin by activation of P2Y receptors. FEBS Lett. 589, 1498-1504. doi: 10.1016/j.febslet.2015.04.044

Bulluck, H., Sirker, A., Loke, Y. K., Garcia-Dorado, D., and Hausenloy, D. J. (2016). Clinical benefit of adenosine as an adjunct to reperfusion in ST-elevation myocardial infarction patients: an updated meta-analysis of randomized controlled trials. Int. J. Cardiol. 202, 228-237. doi: 10.1016/j.ijcard.2015.09.005

Burnstock, G. (1972). Purinergic nerves. Pharmacol. Rev. 24, 509-581.

Burnstock, G. (1981). Pathophysiology of migraine: a new hypothesis. Lancet 317, 1397-1399. doi: 10.1016/S0140-6736(81)92572-1

Burnstock, G. (1999). Release of vasoactive substances from endothelial cells by shear stress and purinergic mechanosensory transduction. J. Anat. 194, 335-342. doi: 10.1046/j.1469-7580.1999.19430335.x

Burnstock, G. (2002). Purinergic signalling and vascular cell proliferation and death. Arterioscler. Thromb. Vasc. Biol. 22, 364-373. doi: 10.1161/hq0302. 105360

Burnstock, G. (2006). Pathophysiology and therapeutic potential of purinergic signaling. Pharmacol. Rev. 58, 58-86. doi: 10.1124/pr.58.1.5

Burnstock, G. (2007). Purine and pyrimidine receptors. Cell. Mol. Life Sci. 64, 1471-1483. doi: 10.1007/s00018-007-6497-0

Burnstock, G. (2008a). Dual control of vascular tone and remodelling by ATP released from nerves and endothelial cells. Pharmacol. Rep. 60, 12-20.

Burnstock, G. (2008b). Purinergic signalling and disorders of the central nervous system. Nat. Rev. Drug Discov. 7, 575-590. doi: 10.1038/nrd2605

Burnstock, G. (2009). Purinergic mechanosensory transduction and visceral pain. Mol. Pain 5:69. doi: 10.1186/1744-8069-5-69

Burnstock, G. (2014a). The erasmus lecture 2012, Academia Europaea. The concept of cotransmission: focus on ATP as a cotransmitter and its significance in health and disease. Eur. Rev. 22, 1-17. doi: 10.1017/S1062798713000586

Burnstock, G. (2014b). Purinergic signalling in endocrine organs. Purinergic Signal. 10, 189-231. doi: 10.1007/s11302-013-9396-x

Burnstock, G. (2014c). Purinergic signalling in the reproductive system in health and disease. Purinergic Signal. 10, 157-187. doi: 10.1007/s11302-013-9399-7

Burnstock, G. (2014d). Purinergic signalling in the urinary tract in health and disease. Purinergic Signal. 10, 103-155. doi: 10.1007/s11302-013-9395-y

Burnstock, G. (2015a). Blood cells: an historical account of the roles of purinergic signalling. Purinergic Signal. 11, 411-434. doi: 10.1007/s11302-015-9462-7

Burnstock, G. (2015b). Physiopathological roles of P2X receptors in the central nervous system. Curr. Med. Chem. 22, 819-844.

Burnstock, G. (2015c). Purinergic signalling in neuroregeneration. Neural Regen. Res. 10, 1919. doi: 10.4103/1673-5374.165300

Burnstock, G. (2016a). An introduction to the roles of purinergic signalling in neurodegeneration, neuroprotection and neuroregeneration. Neuropharmacology 104, 4-17. doi: 10.1016/j.neuropharm.2015. 05.031

Burnstock, G. (2016b). P2X ion channel receptors and inflammation. Purinergic Signal. 12, 59-67. doi: 10.1007/s11302-015-9493-0

Burnstock, G. (2016c). Purinergic receptors and pain - an update. Front. Med. Chem. 9:3-55. doi: 10.2174/9781681082493116090003
Burnstock, G. (2016d). "Purinergic signalling in the gut," in The Enteric Nervous System. 30 Years Later, Vol. 891, eds S. Brierley and M. Costa (Berlin: Springer), 91-112.

Burnstock, G. (2016e). Purinergic signalling: pathophysiology and therapeutic potential. J. Drug Res. Dev. 2. doi: 10.16966/2470-1009.122

Burnstock, G. (2016f). Short- and long-term (trophic) purinergic signalling. Philos. Trans. R. Soc. B Biol. Sci. 371:20150422. doi: 10.1098/rstb.2015.0422

Burnstock, G. (2017). Purinergic signalling in the cardiovascular system. Circ. Res. 120, 207-228. doi: 10.1161/CIRCRESAHA.116.309726

Burnstock, G., Arnett, T. R., and Orriss, I. R. (2013). Purinergic signalling in the musculoskeletal system. Purinergic Signal. 9, 541-572. doi: 10.1007/s11302013-9381-4

Burnstock, G., Brouns, I., Adriaensen, D., and Timmermans, J. P. (2012a). Purinergic signalling in the airways. Pharmacol. Rev. 64, 834-868. doi: 10.1124/ pr.111.005389

Burnstock, G., and Di Virgilio, F. (2013). Purinergic signalling in cancer. Purinergic Signal. 9, 491-540. doi: 10.1007/s11302-013-9372-5

Burnstock, G., Evans, L., and Bailey, M. (2014a). Purinergic signalling in the kidney in health and disease. Purinergic Signal. 10, 71-101. doi: 10.1007/s11302-0139400-5

Burnstock, G., and Kennedy, C. (2011). "P2X receptors in health and disease," in Purine and Pyrimidine Receptor Pharmacology, eds K. A. Jacobson and J. Linden (Burlington: Academic Press), 333-372.

Burnstock, G., and Knight, G. E. (2004). Cellular distribution and functions of P2 receptor subtypes in different systems. Int. Rev. Cytol. 240, 31-304. doi: 10.1016/S0074-7696(04)40002-3

Burnstock, G., Knight, G. E., and Greig, A. V. H. (2012b). Purinergic signalling in healthy and diseased skin. J. Invest. Dermatol. 132, 526-546. doi: 10.1038/jid. 2011.344

Burnstock, G., Krügel, U., Abbracchio, M. P., and Illes, P. (2011). Purinergic signalling: from normal behaviour to pathological brain function. Prog. Neurobiol. 95, 229-274. doi: 10.1016/j.pneurobio.2011.08.006

Burnstock, G., and Loesch, A. (2017). Sympathetic innervation of the kidney in health and disease: emphasis on the role of purinergic cotransmission. Auton. Neurosci. 204, 4-16. doi: 10.1016/j.autneu.2016.05.007

Burnstock, G., and Novak, I. (2013). Purinergic signalling and diabetes. Purinergic Signal. 9, 307-324. doi: 10.1007/s11302-013-9359-2

Burnstock, G., and Pelleg, A. (2015). Cardiac purinergic signalling in health and disease. Purinergic Signal. 11, 1-46. doi: 10.1007/s11302-014-9436-1

Burnstock, G., and Ralevic, V. (2014). Purinergic signaling and blood vessels in health and disease. Pharmacol. Rev. 66, 102-192. doi: 10.1124/pr.113.00 8029

Burnstock, G., and Sawynok, J. (2010). “ATP and adenosine receptors and pain," in Pharmacology of Pain, eds P. Beaulieu, D. Lussier, F. Porreca, and A. H. Dickenson (Seattle: IASP Press), 303-326.

Burnstock, G., Vaughn, B., and Robson, S. (2014b). Purinergic signalling in the liver in health and disease. Purinergic Signal. 10, 51-70. doi: 10.1007/s11302013-9398-8

Burnstock, G., and Verkhratsky, A. (2012). Purinergic Signalling and the Nervous System. Berlin: Springer.

Calvén, J., Akbarshahi, H., Menzel, M., Ayata, C. K., Idzko, M., Bjermer, L., et al. (2015). Rhinoviral stimuli, epithelial factors and ATP signalling contribute to bronchial smooth muscle production of IL-33. J. Transl. Med. 13, 281. doi: 10.1186/s12967-015-0645-3

Calvert, R. C., Banks, F. C., Thompson, C. S., Mikhailidis, D. P., and Morgan, R. J. (2002). Chronic ethanol consumption impairs adrenoceptor- and purinoceptor-mediated relaxations in isolated rat detrusor smooth muscle. BJU. Int 89, 793-794. doi: 10.1046/j.1464-410X.2002.t01-4-02801.x

Calvert, R. C., Khan, M. A., Thompson, C. S., Mikhailidis, D. P., and Burnstock, G. (2008a). A functional study of purinergic signalling in the normal and pathological rabbit corpus cavernosum. BJU Int. 101, 1043-1047. doi: 10.1111/ j.1464-410X.2007.07385.x

Calvert, R. C., Thompson, C. S., and Burnstock, G. (2008b). ATP release from the human ureter on distension and $\mathrm{P} 2 \mathrm{X} 3$ receptor expression on suburothelial sensory nerves. Purinergic Signal. 4, 377-381. doi: 10.1007/s11302-008-9123-1

Cameron, K. O., Kung, D. W., Kalgutkar, A. S., Kurumbail, R. G., Miller, R., Salatto, C. T., et al. (2016). Discovery and preclinical characterization of 6-chloro-5[4-(1-hydroxycyclobutyl)phenyl]-1H-indole-3-carboxylic acid (PF-06409577), 
a direct activator of adenosine monophosphate-activated protein kinase (AMPK), for the potential treatment of diabetic nephropathy. J. Med. Chem. 59, 8068-8081. doi: 10.1021/acs.jmedchem.6b00866

Canda, A. E., Cross, R. L., and Chapple, C. R. (2006). Pharmacology of the lower urinary tract and management of overactive bladder. J. Turk. Ger. Gynecol. Assoc. 7, 146-158.

Canda, A. E., Turna, B., Cinar, G. M., and Nazli, O. (2007). Physiology and pharmacology of the human ureter: basis for current and future treatments. Urol. Int. 78, 289-298. doi: 10.1159/000100830

Cantin, L. D., Bayrakdarian, M., Buon, C., Grazzini, E., Hu, Y. J., Labrecque, J., et al. (2012). Discovery of P2X3 selective antagonists for the treatment of chronic pain. Bioorg. Med. Chem. Lett. 22, 2565-2571. doi: 10.1016/j.bmcl.2012.01.124

Cao, L., Pu, J., Scott, R. H., Ching, J., and Mccaig, C. D. (2015). Physiological electrical signals promote chain migration of neuroblasts by up-regulating P2Y1 purinergic receptors and enhancing cell adhesion. Stem Cell Rev. 11, 75-86. doi: $10.1007 / \mathrm{s} 12015-014-9524-1$

Cao, X., Li, L. P., Wang, Q., Wu, Q., Hu, H. H., Zhang, M., et al. (2013). Astrocytederived ATP modulates depressive-like behaviors. Nat. Med. 19, 773-777. doi: $10.1038 / \mathrm{nm} .3162$

Carneiro, M. V., Americo, T. A., Guimarães, M. Z., and Linden, R. (2016). The prion protein selectively binds to and modulates the content of purinergic receptor P2X4R. Biochem. Biophys. Res. Commun. 472, 293-298. doi: 10.1016/j. bbrc.2016.02.122

Casadó-Anguera, V., Bonaventura, J., Moreno, E., Navarro, G., Cortés, A., Ferré, S., et al. (2016). Evidence for the heterotetrameric structure of the adenosine A2A-dopamine D2 receptor complex. Biochem. Soc. Trans. 44, 595-600. doi: $10.1042 /$ BST20150276

Caseley, E. A., Muench, S. P., Roger, S., Mao, H. J., Baldwin, S. A., and Jiang, L. H. (2014). Non-synonymous single nucleotide polymorphisms in the P2X receptor genes: association with diseases, impact on receptor functions and potential use as diagnosis biomarkers. Int. J. Mol. Sci. 15, 13344-13371. doi: $10.3390 /$ ijms 150813344

Castilhos, L. G., Rezer, J. F., Ruchel, J. B., Thorstenberg, M. L., Jaques, J. A., Schlemmer, J. B., et al. (2015). Effect of Uncaria tomentosa extract on purinergic enzyme activities in lymphocytes of rats submitted to experimental adjuvant arthritis model. BMC Complement. Altern. Med. 15:189. doi: 10.1186/s12906015-0694-4

Catanzaro, J. M., Hueston, C. M., Deak, M. M., and Deak, T. (2014). The impact of the P2X7 receptor antagonist A-804598 on neuroimmune and behavioral consequences of stress. Behav. Pharmacol. 25, 582-598. doi: 10.1097/FBP. 0000000000000072

Cekic, C., and Linden, J. (2016). Purinergic regulation of the immune system. Nat. Rev. Immunol. 16, 177-192. doi: 10.1038/nri.2016.4

Cekic, C., Sag, D., Li, Y., Theodorescu, D., Strieter, R. M., and Linden, J. (2012). Adenosine A2B receptor blockade slows growth of bladder and breast tumor. J. Immunol. 188, 198-205. doi: 10.4049/jimmunol.1101845

Certal, M., Vinhas, A., Pinheiro, A. R., Ferreirinha, F., Costa, M. A., and CorreiaDe-Sá, P. (2015). Novel anti-fibrotic effect of the UTP-sensitive P2Y11 receptor in rat cardiac myofibroblasts. Auton. Neurosci. 192, 72. doi: 10.1016/j.ceca.2015. 08.004

Cha, H. J., Jung, M. S., Ahn, D. W., Choi, J. K., Ock, M. S., Kim, K. S., et al. (2015). Silencing of MUC8 by siRNA increases P2Y2-induced airway inflammation. Am. J. Physiol. Lung. Cell Mol. Physiol. 308, L495-L502. doi: 10.1152/ajplung. 00332.2014

Chan, E. S., and Cronstein, B. N. (2010). Adenosine in fibrosis. Mod. Rheumatol. 20, 114-122. doi: 10.1007/s10165-009-0251-4

Chan, E. S., Montesinos, M. C., Fernandez, P., Desai, A., Delano, D. L., Yee, H., et al. (2006). Adenosine A2A receptors play a role in the pathogenesis of hepatic cirrhosis. Br. J. Pharmacol. 148, 1144-1155. doi: 10.1038/sj.bjp.0706812

Chancellor, M. B., Fowler, C. J., Apostolidis, A., De Groat, W. C., Smith, C. P., Somogyi, G. T., et al. (2008). Drug insight: biological effects of botulinum toxin A in the lower urinary tract. Nat. Clin. Pract. Urol. 5, 319-328. doi: 10.1038/ ncpuro1124

Chandrashekaran, V., Das, S., Seth, R. K., Dattaroy, D., Alhasson, F., Michelotti, G., et al. (2016). Purinergic receptor X7 mediates leptin induced GLUT4 function in stellate cells in nonalcoholic steatohepatitis. Biochim. Biophys. Acta 1862, 32-45. doi: 10.1016/j.bbadis.2015.10.009
Chang, M. Y., Lu, J. K., Tian, Y. C., Chen, Y. C., Hung, C. C., Huang, Y. H., et al. (2011). Inhibition of the P2X7 receptor reduces cystogenesis in PKD. J. Am. Soc. Nephrol. 22, 1696-1706. doi: 10.1681/ASN.2010070728

Chaudhury, A., Dendi, V. S., and Mirza, W. (2016). Colligative property of ATP: implications for enteric purinergic neuromuscular neurotransmission. Front. Physiol. 7:500. doi: 10.3389/fphys.2016.00500

Chaves, M. M., Marques-Da-Silva, C., Monteiro, A. P., Canetti, C., and CoutinhoSilva, R. (2014). Leukotriene B4 modulates P2X7 receptor-mediated Leishmania amazonensis elimination in murine macrophages. J. Immunol. 192, 4765-4773. doi: 10.4049/jimmunol.1301058

Chen, J., Wang, L., Zhang, Y., and Yang, J. (2012). P2Y1 purinoceptor inhibition reduces extracellular signal-regulated protein kinase $1 / 2$ phosphorylation in spinal cord and dorsal root ganglia: implications for cancer-induced bone pain. Acta Biochim. Biophys. Sin. (Shanghai) 44, 367-372. doi: 10.1093/abbs/ gms007

Chen, J. F., Eltzschig, H. K., and Fredholm, B. B. (2013). Adenosine receptors as drug targets - what are the challenges? Nat. Rev. Drug Discov. 12, 265-286. doi: $10.1038 / \mathrm{nrd} 3955$

Chen, X., Molliver, D. C., and Gebhart, G. F. (2010). The P2Y2 receptor sensitizes mouse bladder sensory neurons and facilitates purinergic currents. J. Neurosci. 30, 2365-2372. doi: 10.1523/JNEUROSCI.5462-09.2010

Chen, Y., Zhang, L., Yang, J., Zhang, L., and Chen, Z. (2014). LPS-induced dental pulp inflammation increases expression of ionotropic purinergic receptors in rat trigeminal ganglion. Neuroreport 25, 991-997. doi: 10.1097/WNR. 0000000000000193

Cheng, R. D., Ren, J. J., Zhang, Y. Y., and Ye, X. M. (2014). P2X4 receptors expressed on microglial cells in post-ischemic inflammation of brain ischemic injury. Neurochem. Int. 67, 9-13. doi: 10.1016/j.neuint.2014.01.011

Cheng, Y., Mansfield, K. J., Allen, W., Millard, R. J., Burcher, E., and Moore, K. H. (2013). Correlation between cystometric volumes, ATP release, and $\mathrm{pH}$ in women with overactive bladder versus controls. Neurourol. Urodyn. 32, 969-973. doi: 10.1002/nau.22344

Chesworth, R., Brown, R. M., Kim, J. H., Ledent, C., and Lawrence, A. J. (2016). Adenosine $2 \mathrm{~A}$ receptors modulate reward behaviours for methamphetamine. Addict. Biol. 21, 407-421. doi: 10.1111/adb.12225

Chetty, A., Sharda, A., Warburton, R., Chen, T., Xue, C., Castellot, J., et al. (2016). Effect of a purinergic P2Y6 receptor agonist on airway remodeling and responsiveness in a mouse model of asthma. Am. J. Respir. Crit. Care Med. 193, A2173.

Chiang, D. J., Roychowdhury, S., Bush, K., Mcmullen, M. R., Pisano, S., Niese, K., et al. (2013). Adenosine 2A receptor antagonist prevented and reversed liver fibrosis in a mouse model of ethanol-exacerbated liver fibrosis. PLOS ONE 8:e69114. doi: 10.1371/journal.pone.0069114

Chiu, G. S., Darmody, P. T., Walsh, J. P., Moon, M. L., Kwakwa, K. A., Bray, J. K., et al. (2014). Adenosine through the A2A adenosine receptor increases IL-1 $\beta$ in the brain contributing to anxiety. Brain Behav. Immun. 41, 218-231. doi: 10.1016/j.bbi.2014.05.018

Choi, K. C., Tai, C. J., Tzeng, C. R., Auersperg, N., and Leung, P. C. (2003). Adenosine triphosphate activates mitogen-activated protein kinase in preneoplastic and neoplastic ovarian surface epithelial cells. Biol. Reprod. 68, 309-315. doi: 10.1095/biolreprod.102.006551

Choukèr, A., Thiel, M., Lukashev, D., Ward, J. M., Kaufmann, I., Apasov, S., et al. (2008). Critical role of hypoxia and A2A adenosine receptors in liver tissueprotecting physiological anti-inflammatory pathway. Mol. Med. 14, 116-123. doi: 10.2119/2007-00075.Chouker

Chuang, Y. C., and Chancellor, M. B. (2006). The application of botulinum toxin in the prostate. J. Urol. 176, 2375-2382. doi: 10.1016/j.juro.2006.07.127

Chuo, C. H., Devine, S. M., Scammells, P. J., Krum, H., Christopoulos, A., May, L. T., et al. (2016). VCP746, a novel A1 adenosine receptor biased agonist, reduces hypertrophy in a rat neonatal cardiac myocyte model. Clin. Exp. Pharmacol. Physiol. 43, 976-982. doi: 10.1111/1440-1681.12616

Cicala, C., and Ialenti, A. (2013). Adenosine signaling in airways: toward a promising antiasthmatic approach. Eur. J. Pharmacol. 714, 522-525. doi: 10.1016/j.ejphar.2013.06.033

Cieslak, M., Czarnecka, J., and Roszek, K. (2016). The roles of purinergic signaling in psychiatric disorders. Acta Biochim. Pol. 63, 1-9. doi: 10.18388/abp.2015_ 1004 
Cieslak, M., Czarnecka, J., Roszek, K., and Komoszynski, M. (2015). The role of purinergic signaling in the etiology of migraine and novel antimigraine treatment. Purinergic Signal. 11, 307-316. doi: 10.1007/s11302-015-9453-8

Cieslak, M., and Roszek, K. (2014). Purinergic signaling in the pancreas and the therapeutic potential of ecto-nucleotidases in diabetes. Acta Biochim. Pol. 61, 655-662.

Cieslak, M., Wojtczak, A., and Komoszynski, M. (2017). Role of the purinergic signaling in epilepsy. Pharmacol. Rep. 69, 130-138. doi: 10.1016/j.pharep.2016. 09.018

Ciruela, F., Fernández-Dueñas, V., Contreras, F., Arnau, J. M., Menchón, J. M., Vallano, A., et al. (2015). "Adenosine in the neurobiology of schizophrenia: potential adenosine receptor-based pharmacotherapy," in Psychiatry and Neuroscience Update: Bridging the Divide, eds P. Á Gargiulo and H. L. M. Arroyo (Cham: Springer International Publishing), 375-388.

Cisneros-Mejorado, A., Gottlieb, M., Cavaliere, F., Magnus, T., Koch-Nolte, F., Scemes, E., et al. (2015a). Blockade of P2X7 receptors or pannexin-1 channels similarly attenuates postischemic damage. J. Cereb. Blood Flow Metab. 35, 843-850. doi: 10.1038/jcbfm.2014.262

Cisneros-Mejorado, A., Perez-Samartin, A., Gottlieb, M., and Matute, C. (2015b). ATP signaling in brain: release, excitotoxicity and potential therapeutic targets. Cell. Mol. Neurobiol. 35, 1-6. doi: 10.1007/s10571-014-0092-3

Clouet, S., Di Pietrantonio, L., Daskalopoulos, E. P., Esfahani, H., Horckmans, M., Vanorlé, M., et al. (2016). Loss of mouse P2Y6 nucleotide receptor is associated with physiological macrocardia and amplified pathological cardiac hypertrophy. J. Biol. Chem. 291, 15841-15852. doi: 10.1074/jbc.M115.684118

Cockayne, D. A., Hamilton, S. G., Zhu, Q.-M., Dunn, P. M., Zhong, Y., Novakovic, S., et al. (2000). Urinary bladder hyporeflexia and reduced painrelated behaviour in P2X3-deficient mice. Nature 407, 1011-1015. doi: 10.1038/ 35039519

Cohen, S. B., Gill, S. S., Baer, G. S., Leo, B. M., Scheld, W. M., and Diduch, D. R. (2004). Reducing joint destruction due to septic arthrosis using an adenosine $2 \mathrm{~A}$ receptor agonist. J. Orthop. Res. 22, 427-435. doi: 10.1016/j.orthres.2003.08.011

Corciulo, C., Lendhey, M., Wilder, T., Schoen, H., Cornelissen, A. S., Chang, G., et al. (2017). Endogenous adenosine maintains cartilage homeostasis and exogenous adenosine inhibits osteoarthritis progression. Nat. Commun. 8:15019. doi: 10.1038/ncomms15019

Corrêa, G., Almeida, L. C., Moreira-Souza, A. C., Savio, L. E., Takiya, C. M., Marques-Da-Silva, C., et al. (2017). Inflammatory early events associated to the role of P2X7 receptor in acute murine toxoplasmosis. Immunobiology 222, 676-683. doi: 10.1016/j.imbio.2016.12.007

Corrêa, G., Lindenberg, C. D. A., Fernandes-Santos, C., Gandini, M., Petitinga, P. F., Coutinho-Silva, R., et al. (2016). The purinergic receptor P2X7 role in control of Dengue virus-2 infection and cytokine/chemokine production in infected human monocytes. Immunobiology 221, 794-802. doi: 10.1016/j.imbio. 2016.02.003

Corso, L., Cavallero, A., Baroni, D., Garbati, P., Prestipino, G., Bisti, S., et al. (2016). Saffron reduces ATP-induced retinal cytotoxicity by targeting P2X7 receptors. Purinergic Signal. 12, 161-174. doi: 10.1007/s11302-015-9490-3

Costello, J. C., Rosenthal, A. K., Kurup, I. V., Masuda, I., Medhora, M., and Ryan, L. M. (2011). Parallel regulation of extracellular ATP and inorganic pyrophosphate: roles of growth factors, transduction modulators, and ANK. Connect. Tissue Res. 52, 139-146. doi: 10.3109/03008207.2010.491928

Côté, N., El Husseini, D., Pepin, A., Guauque-Olarte, S., Ducharme, V., BouchardCannon, P., et al. (2012). ATP acts as a survival signal and prevents the mineralization of aortic valve. J. Mol. Cell Cardiol. 52, 1191-1202. doi: 10.1016/ j.yjmcc.2012.02.003

Coughlan, K. S., Mitchem, M. R., Hogg, M. C., and Prehn, J. H. (2015). "Preconditioning" with latrepirdine, an adenosine 5 '-monophosphate-activated protein kinase activator, delays amyotrophic lateral sclerosis progression in SOD1(G93A) mice. Neurobiol. Aging 36, 1140-1150. doi: 10.1016/j. neurobiolaging.2014.09.022

Cristalli, G., Vittori, S., Thompson, R. D., Padgett, W. L., Shi, D., Daly, J. W., et al. (1994). Inhibition of platelet aggregation by adenosine receptor agonists. Naunyn Schmiedebergs Arch. Pharmacol. 349, 644-650.

Crossland, R. F., Durgan, D. J., Lloyd, E. E., Phillips, S. C., Reddy, A. K., Marrelli, S. P., et al. (2013). A new rodent model for obstructive sleep apnea: effects on ATP-mediated dilations in cerebral arteries. Am. J. Physiol. Regul. Integr. Comp. Physiol. 305, R334-R342. doi: 10.1152/ajpregu.00244.2013
Cruz, F. (2013). Targets for botulinum toxin in the lower urinary tract. Neurourol. Urodyn. 33, 31-38. doi: 10.1002/nau.22445

Csóka, B., Németh, Z. H., Törö, G., Idzko, M., Zech, A., Koscsó, B., et al. (2015). Extracellular ATP protects against sepsis through macrophage P2X7 purinergic receptors by enhancing intracellular bacterial killing. FASEB J. 29, 3626-3637. doi: 10.1096/fj.15-272450

Cunha, M. P., Pazini, F. L., Rosa, J. M., Ramos-Hryb, A. B., Oliveira, Á, Kaster, M. P., et al. (2015). Creatine, similarly to ketamine, affords antidepressant-like effects in the tail suspension test via adenosine A1 and A2A receptor activation. Purinergic Signal. 11, 215-227. doi: 10.1007/s11302-015-9446-7

Cunha, R. (2015). Hippocampal adenosine A2A receptor up-regulation isnecessary and sufficient to trigger memory dysfunction inAlzheimer's disease. J. Neurochem. 134, 322.

Cunha, R. A. (2016). How does adenosine control neuronal dysfunction and neurodegeneration? J. Neurochem. 139, 1019-1055. doi: 10.1111/jnc.13724

da Rocha Lapa, F., Da Silva, M. D., De Almeida Cabrini, D., and Santos, A. R. (2012). Anti-inflammatory effects of purine nucleosides, adenosine and inosine, in a mouse model of pleurisy: evidence for the role of adenosine A2 receptors. Purinergic Signal. 8, 693-704. doi: 10.1007/s11302-012-9299-2

da Rocha Lapa, F., Júnior, S. J. M., Cerutti, M. L., and Santos, A. R. S. (2014). "Pharmacology of adenosine receptors and their signaling role in immunity and inflammation," in Pharmacology and Therapeutics, ed. S. J. T. Gowder (Rijeka: InTech), 85-130.

da Silva, M. V., Marosti, A. R., Mendes, C. E., Palombit, K., and Castelucci, P. (2015). Differential effects of experimental ulcerative colitis on P2X7 receptor expression in enteric neurons. Histochem. Cell Biol. 143, 171-184. doi: 10.1007/ s00418-014-1270-6

da Silva, M. V., Marosti, A. R., Mendes, C. E., Palombit, K., and Castelucci, P. (2017). Submucosal neurons and enteric glial cells expressing the P2X7 receptor in rat experimental colitis. Acta Histochem. 119, 481-494. doi: 10.1016/j.acthis. 2017.05.001

Dai, S. S., and Zhou, Y. G. (2011). Adenosine 2A receptor: a crucial neuromodulator with bidirectional effect in neuroinflammation and brain injury. Rev. Neurosci. 22, 231-239. doi: 10.1515/RNS.2011.020

Dai, Y., Zhang, Y., Phatarpekar, P., Mi, T., Zhang, H., Blackburn, M. R., et al. (2009). Adenosine signaling, priapism and novel therapies. J. Sex. Med. 6(Suppl. 3), 292-301. doi: 10.1111/j.1743-6109.2008.01187.x

D’Alimonte, I., Nargi, E., Zuccarini, M., Lanuti, P., Di Iorio, P., Giuliani, P., et al. (2015). Potentiation of temozolomide antitumor effect by purine receptor ligands able to restrain the in vitro growth of human glioblastoma stem cells. Purinergic Signal. 11, 331-346. doi: 10.1007/s11302-0159454-7

Daneshgari, F., Liu, G., and Imrey, P. B. (2006). Time dependent changes in diabetic cystopathy in rats include compensated and decompensated bladder function. J. Urol. 176, 380-386. doi: 10.1016/S0022-5347(06)00582-9

Daniele, S., Zappelli, E., Natali, L., Martini, C., and Trincavelli, M. L. (2014). Modulation of $\mathrm{A} 1$ and $\mathrm{A} 2 \mathrm{~B}$ adenosine receptor activity: a new strategy to sensitise glioblastoma stem cells to chemotherapy. Cell. Death Dis. 5, e1539. doi: $10.1038 /$ cddis. 2014.487

Danquah, W., Meyer-Schwesinger, C., Rissiek, B., Pinto, C., Serracant-Prat, A., Amadi, M., et al. (2016). Nanobodies that block gating of the P2X7 ion channel ameliorate inflammation. Sci. Transl. Med. 8, 366ra162. doi: 10.1126/ scitranslmed.aaf8463

Darville, T., Welter-Stahl, L., Cruz, C., Sater, A. A., Andrews, C. W. Jr., and Ojcius, D. M. (2007). Effect of the purinergic receptor P2X7 on Chlamydia infection in cervical epithelial cells and vaginally infected mice. J. Immunol. 179, 3707-3714. doi: 10.4049/jimmunol.179.6.3707

Davies, J., Li, H., Karmouty-Quintana, H., Chen, N. Y., Weng, T., Molina, J., et al. (2016). Extracellular adenosine is detrimental in lung recovery following hyperoxic lung injury in a murine model of bronchopulmonary dysplasia. Am. J. Respir. Crit. Care Med. 193, A3850.

de Azevedo, M. I., Ferreiro, L., Da Silva, A. S., Tonin, A. A., Monteiro, D. U., Casali, E. A., et al. (2016). Participation of purines in the modulation of inflammatory response in rats experimentally infected by Cryptococcus neoformans. Microb. Pathog. 99, 36-40. doi: 10.1016/j.micpath.2016.07.015

de Diego-Garcia, L., Ramírez-Escudero, M., Sebastián-Serrano, A., DiazHernández, J. I., Pintor, J., Lucas, J. J., et al. (2017). Regulation of proteasome activity by $\mathrm{P} 2 \mathrm{Y} 2$ receptor underlies the neuroprotective effects of extracellular 
nucleotides. Biochim. Biophys. Acta 1863, 43-51. doi: 10.1016/j.bbadis.2016. 10.012

de Lera Ruiz, M., Lim, Y. H., and Zheng, J. (2014). Adenosine A2A receptor as a drug discovery target. J. Med. Chem. 57, 3623-3650. doi: 10.1021/jm4011669

De Luca, L., Capranzano, P., Patti, G., and Parodi, G. (2016). Switching of platelet P2Y12 receptor inhibitors in patients with acute coronary syndromes undergoing percutaneous coronary intervention: review of the literature and practical considerations. Am. Heart J. 176, 44-52. doi: 10.1016/j.ahj.2016.03.006

De Mattei, M., Varani, K., Masieri, F. F., Pellati, A., Ongaro, A., Fini, M., et al. (2009). Adenosine analogs and electromagnetic fields inhibit prostaglandin E2 release in bovine synovial fibroblasts. Osteoarthritis Cartilage 17, 252-262. doi: $10.1016 /$ j.joca.2008.06.002

Deiteren, A., Van Der Linden, L., De Wit, A., Ceuleers, H., Buckinx, R., Timmermans, J. P., et al. (2015). P2X3 receptors mediate visceral hypersensitivity during acute chemically-induced colitis and in the postinflammatory phase via different mechanisms of sensitization. PLOS ONE 10:e0123810. doi: 10.1371/journal.pone.0123810

Della Latta, V., Cabiati, M., Rocchiccioli, S., Del Ry, S., and Morales, M. A. (2013). The role of the adenosinergic system in lung fibrosis. Pharmacol. Res. 76, 182-189. doi: 10.1016/j.phrs.2013.08.004

Densmore, J. C., Schaid, T. R., Jeziorczak, P. M., Medhora, M., Audi, S., Nayak, S., et al. (2017). Lung injury pathways: adenosine receptor $2 \mathrm{~B}$ signaling limits development of ischemic bronchiolitis obliterans organizing pneumonia. Exp. Lung Res. 43, 38-48. doi: 10.1080/01902148.2017.1286697

De Oliveira Moreira, D., Santo Neto, H., and Marques, M. J. (2017). P2Y2 purinergic receptors are highly expressed in cardiac and diaphragm muscles of mdx mice, and their expression is decreased by suramin. Muscle Nerve 55, 116-121. doi: 10.1002/mus.25199

DeOliveira, C. C., Paiva Caria, C. R., Ferreira Gotardo, E. M., Ribeiro, M. L., and Gambero, A. (2017). Role of A1 and A2A adenosine receptor agonists in adipose tissue inflammation induced by obesity in mice. Eur. J. Pharmacol. 799, 154-159. doi: 10.1016/j.ejphar.2017.02.017

Deplano, S., Cook, H. T., Russell, R., Franchi, L., Schneiter, S., Bhangal, G., et al. (2013). P2X7 receptor-mediated Nlrp3-inflammasome activation is a genetic determinant of macrophage-dependent crescentic glomerulonephritis. J. Leukoc. Biol. 93, 127-134. doi: 10.1189/jlb.0612284

Diezmos, E. F., Bertrand, P. P., and Liu, L. (2016). Purinergic signaling in gut inflammation: the role of connexins and pannexins. Front. Neurosci. 10:311. doi: $10.3389 /$ fnins.2016.00311

Di Virgilio, F. (2015). P2X receptors and inflammation. Curr. Med. Chem. 22, 866-877. doi: 10.2174/0929867322666141210155311

Di Virgilio, F., and Adinolfi, E. (2017). Extracellular purines, purinergic receptors and tumor growth. Oncogene 36, 293-303. doi: 10.1038/onc.2016.206

Di Virgilio, F., and Vuerich, M. (2015). Purinergic signaling in the immune system. Auton. Neurosci. 191, 117-123. doi: 10.1016/j.autneu.2015.04.011

Djerada, Z., Feliu, C., Richard, V., and Millart, H. (2017). Current knowledge on the role of $\mathrm{P} 2 \mathrm{Y}$ receptors in cardioprotection against ischemia-reperfusion. Pharmacol. Res. 118, 5-18. doi: 10.1016/j.phrs.2016.08.009

do Carmo, G. M., Doleski, P. H., De Sá, M. F., Grando, T. H., Bottari, N. B., Leal, D. B., et al. (2017). Purinergic enzymatic activities in lymphocytes and cardiomyocytes of mice acutely infected by Trypanosoma cruzi modulating the inflammatory responses. Exp. Parasitol. 175, 44-50. doi: 10.1016/j.exppara. 2017.02.002

Domercq, M., Perez-Samartin, A., Aparicio, D., Alberdi, E., Pampliega, O., and Matute, C. (2010). P2X7 receptors mediate ischemic damage to oligodendrocytes. Glia 58, 730-740. doi: 10.1002/glia.20958

Dong, F., Yang, X. J., Jiang, T. B., and Chen, Y. (2016). Ischemia triggered ATP release through Pannexin-1 channel by myocardial cells activates sympathetic fibers. Microvasc. Res. 104, 32-37. doi: 10.1016/j.mvr.2015.11.005

Dragicevic, E., Schiemann, J., and Liss, B. (2015). Dopamine midbrain neurons in health and Parkinson's disease: emerging roles of voltage-gated calcium channels and ATP-sensitive potassium channels. Neuroscience 284, 798-814. doi: 10.1016/j.neuroscience.2014.10.037

Dranoff, J. A., Kruglov, E. A., Abreu-Lanfranco, O., Nguyen, T., Arora, G., and Jain, D. (2007). Prevention of liver fibrosis by the purinoceptor antagonist pyridoxal-phosphate-6-azophenyl-2 $2^{\prime}, 4^{\prime}$-disulfonate (PPADS). In Vivo 21, 957-965.
Du, D., Jiang, M., Liu, M., Wang, J., Xia, C., Guan, R., et al. (2015). Microglial P2X7 receptor in the hypothalamic paraventricular nuclei contributes to sympathoexcitatory responses in acute myocardial infarction rat. Neurosci. Lett. 587, 22-28. doi: 10.1016/j.neulet.2014.12.026

Du, X., Ou, X., Song, T., Zhang, W., Cong, F., Zhang, S., et al. (2015). Adenosine A2B receptor stimulates angiogenesis by inducing VEGF and eNOS in human microvascular endothelial cells. Exp. Biol. Med. 240, 1472-1479. doi: 10.1177/ 1535370215584939

Dziubina, A., Szmyd, K., Zygmunt, M., Sapa, J., Dudek, M., Filipek, B., et al. (2016). Evaluation of antidepressant-like and anxiolytic-like activity of purinedionederivatives with affinity for adenosine A2A receptors in mice. Pharmacol. Rep. 68, 1285-1292. doi: 10.1016/j.pharep.2016.07.008

Eckle, T., Koeppen, M., and Eltzschig, H. K. (2009). Role of extracellular adenosine in acute lung injury. Physiology 24, 298-306. doi: 10.1152/physiol.00022. 2009

El-Hashim, A. Z., Mathew, S., and Spina, D. (2016). The Al adenosine receptor is a novel inhibitory receptor that regulates the cough reflex via a central mechanism of action. Am. J. Respir. Crit. Care Med. 193, A6003-A6003.

Elsherbiny, N. M., Ahmad, S., Naime, M., Elsherbini, A. M., Fulzele, S., Al-Gayyar, M. M., et al. (2013a). ABT-702, an adenosine kinase inhibitor, attenuates inflammation in diabetic retinopathy. Life Sci. 93, 78-88. doi: 10.1016/j.lfs.2013. 05.024

Elsherbiny, N. M., Naime, M., Ahmad, S., Elsherbini, A. M., Mohammad, S., Fulzele, S., et al. (2013b). Potential roles of adenosine deaminase-2 in diabetic retinopathy. Biochem. Biophys. Res. Commun. 436, 355-361. doi: 10.1016/j.bbrc. 2013.05.023

Engel, T. (2016). Purinergic signaling-induced neuroinflammation and status epilepticus. Expert. Rev. Neurother. 16, 735-737. doi: 10.1586/14737175.2016. 1164036

Engel, T., Alves, M., Sheedy, C., and Henshall, D. C. (2016). ATPergic signalling during seizures and epilepsy. Neuropharmacology 104, 140-153. doi: 10.1016/j. neuropharm.2015.11.001

Erb, L., Cao, C., Ajit, D., and Weisman, G. A. (2015). P2Y receptors in Alzheimer's disease. Biol. Cell 107, 1-21. doi: 10.1111/boc.201400043

Erlinge, D., and Burnstock, G. (2008). P2 receptors in cardiovascular regulation and disease. Purinergic Signal. 4, 1-20. doi: 10.1007/s11302-007-9078-7

Escudero, C., Casanello, P., and Sobrevia, L. (2008). Human equilibrative nucleoside transporters 1 and 2 may be differentially modulated by A2B adenosine receptors in placenta microvascular endothelial cells from pre-eclampsia. Placenta 29, 816-825. doi: 10.1016/j.placenta.2008.06.014

Escudero, C., Roberts, J. M., Myatt, L., and Feoktistov, I. (2014). Impaired adenosine-mediated angiogenesis in preeclampsia: potential implications for fetal programming. Front. Pharmacol. 5:134. doi: 10.3389/fphar.2014.00134

Eser, A., Colombel, J. F., Rutgeerts, P., Vermeire, S., Vogelsang, H., Braddock, M., et al. (2015). Safety and efficacy of an oral inhibitor of the purinergic receptor P2X7 in adult patients with moderately to severely active Crohn's disease: a randomized placebo-controlled, double-blind, phase IIa study. Inflamm. Bowel Dis. 21, 2247-2253. doi: 10.1097/MIB.0000000000000514

Essawy, S. S., Tawfik, M. K., and Korayem, H. E. (2017). Effects of adenosine receptor antagonists in MPTP mouse model of Parkinson's disease: mitochondrial DNA integrity. Arch. Med. Sci. 13, 659-669. doi: 10.5114/aoms. 2017.67284

Esther, C. R. Jr., Olsen, B. M., Lin, F. C., Fine, J., and Boucher, R. C. (2013). Exhaled breath condensate adenosine tracks lung function changes in cystic fibrosis. Am. J. Physiol. Lung. Cell Mol. Physiol. 304, L504-L509. doi: 10.1152/ajplung. 00344.2012

Esther, C. R., Muhlebach, M. M., Turkovic, L., Rosenow, T., Boucher, R. C., and Stick, S. M. (2015). Increased airway adenosine metabolism and oxidative stress in early cystic fibrosis lung disease. Am. J. Respir. Crit. Care Med. 191, A1032.

Eun, S. Y., Ko, Y. S., Park, S. W., Chang, K. C., and Kim, H. J. (2015). P2Y2 nucleotide receptor-mediated extracellular signal-regulated kinases and protein kinase $\mathrm{C}$ activation induces the invasion of highly metastatic breast cancer cells. Oncol. Rep. 34, 195-202. doi: 10.3892/or.2015.3972

Eyo, U. B., Peng, J., Swiatkowski, P., Mukherjee, A., Bispo, A., and Wu, L. J. (2014). Neuronal hyperactivity recruits microglial processes via neuronal NMDA receptors and microglial $\mathrm{P} 2 \mathrm{Y} 12$ receptors after status epilepticus. J. Neurosci. 34, 10528-10540. doi: 10.1523/JNEUROSCI.0416-14.2014 
Faas, M. M., Sáez, T., and De Vos, P. (2017). Extracellular ATP and adenosine: the Yin and Yang in immune responses? Mol. Aspects Med. 55, 9-19. doi: 10.1016/j.mam.2017.01.002

Fabiyi, A. C., and Brading, A. F. (2006). The use of the isolated mouse whole bladder for investigating bladder overactivity. J. Pharmacol. Exp. Ther. 319, 1386-1394. doi: 10.1124/jpet.106.108902

Falk, S., Schwab, S. D., Frosig-Jorgensen, M., Clausen, R. P., Dickenson, A. H., and Heegaard, A. M. (2015). P2X7 receptor-mediated analgesia in cancer-induced bone pain. Neuroscience 291, 93-105. doi: 10.1016/j.neuroscience.2015.02.011

Fan, Y., Kong, H., Ye, X., Ding, J., and Hu, G. (2016). ATP-sensitive potassium channels: uncovering novel targets for treating depression. Brain Struct. Funct. 221, 3111-3122. doi: 10.1007/s00429-015-1090-z

Fang, J., Chen, X., Wang, S., Xie, T., Du, X., Liu, H., et al. (2015). The expression of P2X7 receptors in EPCs and their potential role in the targeting of EPCs to brain gliomas. Cancer Biol. Ther. 16, 498-510. doi: 10.1080/15384047.2015.1016663

Faria, M., Timóteo, M. A., Lafuente-De-Carvalho, M., and Correia-De-Sá, P. (2010). P2 purinoceptor subtyope changes in patients with vasculogenic erectile dysfunction. Purinergic Signal. 6, S115.

Fasullo, M., and Endres, L. (2015). Nucleotide salvage deficiencies, DNA damage and neurodegeneration. Int. J. Mol. Sci. 16, 9431-9449. doi: 10.3390/ ijms16059431

Fathalla, A. M., Soliman, A. M., Ali, M. H., and Moustafa, A. A. (2016). Adenosine $\mathrm{A} 2 \mathrm{~A}$ receptor blockade prevents rotenone-induced motor impairment in a rat model of Parkinsonism. Front. Behav. Neurosci. 10:35. doi: 10.3389/fnbeh.2016. 00035

Fausther, M., Sheung, N., Saiman, Y., Bansal, M. B., and Dranoff, J. A. (2012). Activated hepatic stellate cells upregulate transcription of ecto- $5^{\prime}$ nucleotidase/CD73 via specific SP1 and SMAD promoter elements. Am. J. Physiol. Gastrointest. Liver Physiol. 303, G904-G914. doi: 10.1152/ajpgi.00015. 2012

Fehm, T., Zwirner, M., Wallwiener, D., Seeger, H., and Neubauer, H. (2012). Antitumor activity of zoledronic acid in primary breast cancer cells determined by the ATP tumor chemosensitivity assay. BMC Cancer 12:308. doi: 10.1186/ 1471-2407-12-308

Feng, L., Sun, X., Csizmadia, E., Han, L., Bian, S., Murakami, T., et al. (2011). Vascular CD39/ENTPD1 directly promotes tumor cell growth by scavenging extracellular adenosine triphosphate. Neoplasia 13, 206-216. doi: 10.1593/neo. 101332

Feng, Y. H., Li, X., Wang, L., Zhou, L., and Gorodeski, G. I. (2006). A truncated $\mathrm{P} 2 \mathrm{X} 7$ receptor variant (P2X7-j) endogenously expressed in cervical cancer cells antagonizes the full-length P2X7 receptor through hetero-oligomerization. J. Biol. Chem. 281, 17228-17237. doi: 10.1074/jbc.M602999200

Fern, R. F., Matute, C., and Stys, P. K. (2014). White matter injury: ischemic and nonischemic. Glia 62, 1780-1789. doi: 10.1002/glia.22722

Fernando, S. L., Saunders, B. M., Sluyter, R., Skarratt, K. K., Goldberg, H., Marks, G. B., et al. (2007). A polymorphism in the P2X7 gene increases susceptibility to extrapulmonary tuberculosis. Am. J. Respir. Crit. Care Med. 175, 360-366. doi: $10.1164 /$ rccm.200607-970OC

Ferrante, A., Martire, A., Pepponi, R., Varani, K., Vincenzi, F., Ferraro, L., et al. (2014). Expression, pharmacology and functional activity of adenosine A1 receptors in genetic models of Huntington's disease. Neurobiol. Dis. 71, 193-204. doi: 10.1016/j.nbd.2014.08.013

Ferrari, D., Bianchi, N., Eltzschig, H. K., and Gambari, R. (2016a). MicroRNAs modulate the purinergic signaling network. Trends Mol. Med. 22, 905-918. doi: 10.1016/j.molmed.2016.08.006

Ferrari, D., Mcnamee, E. N., Idzko, M., Gambari, R., and Eltzschig, H. K. (2016b). Purinergic signaling during immune cell trafficking. Trends Immunol. 37, 399-411. doi: 10.1016/j.it.2016.04.004

Ferrari, D., Vitiello, L., Idzko, M., and La Sala, A. (2015). Purinergic signaling in atherosclerosis. Trends Mol. Med. 21, 184-192. doi: 10.1016/j.molmed.2014. 12.008

Ferré, S., Bonaventura, J., Tomasi, D., Navarro, G., Moreno, E., Cortés, A., et al. (2016). Allosteric mechanisms within the adenosine A2A-dopamine D2 receptor heterotetramer. Neuropharmacology 104, 154-160. doi: 10.1016/ j.neuropharm.2015.05.028

Ferrée, S. (2016). Mechanisms of the psychostimulant effects of caffeine: implications for substance use disorders. Psychopharmacology 233, 1963-1979. doi: $10.1007 / \mathrm{s} 00213-016-4212-2$
Festugato, M. (2015). Adenosine: an endogenous mediator in the pathogenesis of psoriasis. An. Bras. Dermatol. 90, 862-867. doi: 10.1590/abd1806-4841. 20153689

Fiebich, B. L., Akter, S., and Akundi, R. S. (2014). The two-hit hypothesis for neuroinflammation: role of exogenous ATP in modulating inflammation in the brain. Front. Cell Neurosci. 8:260. doi: 10.3389/fncel.2014.00260

Figliuolo, V. R., Chaves, S. P., Savio, L. E. B., Thorstenberg, M. L. P., Machado Salles, E., Takiya, C. M., et al. (2017a). The role of the P2X7 receptor in murine cutaneous leishmaniasis: aspects of inflammation and parasite control. Purinergic Signal. 13, 143-152. doi: 10.1007/s11302-016-9544-1

Figliuolo, V. R., Savio, L. E. B., Safya, H., Nanini, H., Bernardazzi, C., Abalo, A., et al. (2017b). P2X7 receptor promotes intestinal inflammation in chemically induced colitis and triggers death of mucosal regulatory T cells. Biochim. Biophys. Acta 1863, 1183-1194. doi: 10.1016/j.bbadis.2017.03.004

Figueiredo, A. B., Souza-Testasicca, M. C., and Afonso, L. C. (2016). Purinergic signaling and infection by Leishmania: a new approach to evasion of the immune response. Biomed. J. 39, 244-250. doi: 10.1016/j.bj.2016.08.004

Fischer, T., Rotermund, N., Lohr, C., and Hirnet, D. (2012). P2Y1 receptor activation by photolysis of caged ATP enhances neuronal network activity in the developing olfactory bulb. Purinergic Signal. 8, 191-198. doi: 10.1007/s11302011-9286-z

Fish, R. S., Klootwijk, E., Tam, F. W. K., Kleta, R., Wheeler, D. C., Unwin, R. J., et al. (2013). ATP and arterial calcification. Eur. J. Clin. Invest. 43, 405-412. doi: 10.1111/eci.12055

Fishman, P., Ochaion, A., Cohen, S., Patoka, R., Barer, F., and Bar-Yehuda, S. (2008). The anti-inflammatory effect of A3 adenosine receptor agonists: a novel targeted therapy for rheumatoid arthritis. Expert Opin. Investig. Drugs 16, 1601-1613. doi: 10.1517/13543784.16.10.1601

Fonseca, B., Martinez-Aguila, A., De Lara, M. J. P., and Pintor, J. (2017). Diadenosine tetraphosphate as a potential therapeutic nucleotide to treat glaucoma. Purinergic Signal. 13, 171-177. doi: 10.1007/s11302-016-9547-y

Ford, A. P., and Cockayne, D. A. (2011). ATP and P2X purinoceptors in urinary tract disorders. Handb. Exp. Pharmacol. 202, 485-526. doi: 10.1007/978-3-64216499-6_22

Ford, A. P., and Undem, B. J. (2013). The therapeutic promise of ATP antagonism at $\mathrm{P} 2 \mathrm{X} 3$ receptors in respiratory and urological disorders. Front. Cell Neurosci. 7:267. doi: $10.3389 /$ fncel.2013.00267

Ford, A. P., Undem, B. J., Birder, L. A., Grundy, D., Pijacka, W., and Paton, J. F. (2015). P2X3 receptors and sensitization of autonomic reflexes. Auton. Neurosci. 191, 16-24. doi: 10.1016/j.autneu.2015.04.005

Förster, D., and Reiser, G. (2015). Supportive or detrimental roles of P2Y receptors in brain pathology?-The two faces of P2Y receptors in stroke and neurodegeneration detected in neural cell and in animal model studies. Purinergic Signal. 11, 441-454. doi: 10.1007/s11302-015-9471-6

Fotino, C., Vergani, A., Fiorina, P., and Pileggi, A. (2015). P2X receptors and diabetes. Curr. Med. Chem. 22, 891-901. doi: 10.2174/ 0929867321666141012173520

Fragakis, N., Antoniadis, A. P., Saviano, M., Vassilikos, V., and Pappone, C. (2015). The use of adenosine and adenosine triphosphate testing in the diagnosis, risk stratification and management of patients with syncope: current evidence and future perspectives. Int. J. Cardiol. 183, 267-273. doi: 10.1016/j.ijcard.2015. 01.089

Franco, M., Bautista-Perez, R., Cano-Martinez, A., Pacheco, U., Santamaria, J., Del Valle-Mondragon, L., et al. (2017). Physiopathological implications of P2X1 and P2X7 receptors in regulation of glomerular hemodynamics in angiotensin II-induced hypertension. Am. J. Physiol. Renal Physiol. 313, F9-F19. doi: 10. 1152/ajprenal.00663.2016

Franklin, K. M., Asatryan, L., Jakowec, M. W., Trudell, J. R., Bell, R. L., and Davies, D. L. (2014). P2X4 receptors (P2X4Rs) represent a novel target for the development of drugs to prevent and/or treat alcohol use disorders. Front. Neurosci. 8:176. doi: 10.3389/fnins.2014.00176

Fransson, A., Järlebark, L. E., and Ulfendahl, M. (2009). In vivo infusion of UTP and uridine to the deafened guinea pig inner ear: effects on response thresholds and neural survival. J. Neurosci. Res. 87, 1712-1717. doi: 10.1002/jnr.21969

Frenguelli, B. G., and Wall, M. J. (2016). Combined electrophysiological and biosensor approaches to study purinergic regulation of epileptiform activity in cortical tissue. J. Neurosci. Methods 260, 202-214. doi: 10.1016/j.jneumeth.2015. 09.011 
Friebe, D., Yang, T., Schmidt, T., Borg, N., Steckel, B., Ding, Z., et al. (2014). Purinergic signaling on leukocytes infiltrating the LPS-injured lung. PLOS ONE 9:e95382. doi: 10.1371/journal.pone.0095382

Frontini, A. V., Cd, D. L. V. E., Nicolorich, M. V., Naves, A., Schwarzbaum, P., and Venera, G. D. (2011). In vivo effects of adenosine $5^{\prime}$-triphosphate on rat preneoplastic liver. Medicina 71, 139-145.

$\mathrm{Fu}, \mathrm{S}$., and Davies, K. P. (2015). Opiorphin-dependent upregulation of CD73 (a key enzyme in the adenosine signaling pathway) in corporal smooth muscle cells exposed to hypoxic conditions and in corporal tissue in pre-priapic sickle cell mice. Int. J. Impot. Res. 27, 140-145. doi: 10.1038/ijir.2015.5

Fu, W., Mccormick, T., Qi, X., Luo, L., Zhou, L., Li, X., et al. (2009). Activation of P2X7-mediated apoptosis Inhibits DMBA/TPA-induced formation of skin papillomas and cancer in mice. BMC Cancer 9:114. doi: 10.1186/1471-24079-114

Fuller, S. J., Stokes, L., Skarratt, K. K., Gu, B. J., and Wiley, J. S. (2009). Genetics of the P2X7 receptor and human disease. Purinergic Signal. 5, 257-262. doi: 10.1007/s11302-009-9136-4

Fumagalli, M., Lecca, D., and Abbracchio, M. P. (2016). CNS remyelination as a novel reparative approach to neurodegenerative diseases: the roles of purinergic signaling and the P2Y-like receptor GPR17. Neuropharmacology 104, 82-93. doi: 10.1016/j.neuropharm.2015.10.005

Füredi, R., Bölcskei, K., Szolcsányi, J., and Pethö, G. (2010). Comparison of the peripheral mediator background of heat injury- and plantar incision-induced drop of the noxious heat threshold in the rat. Life Sci. 86, 244-250. doi: 10.1016/ j.lfs.2009.12.010

Furlan-Freguia, C., Marchese, P., Gruber, A., Ruggeri, Z. M., and Ruf, W. (2011). $\mathrm{P} 2 \mathrm{X} 7$ receptor signaling contributes to tissue factor-dependent thrombosis in mice. J. Clin. Invest. 121, 2932-2944. doi: 10.1172/JCI46129

Furlong, T. M., Supit, A. S., Corbit, L. H., Killcross, S., and Balleine, B. W. (2015). Pulling habits out of rats: adenosine $2 \mathrm{~A}$ receptor antagonism in dorsomedial striatum rescues meth-amphetamine-induced deficits in goal-directed action. Addict. Biol. 22, 172-183. doi: 10.1111/adb.12316

Gan, M., Moussaud, S., Jiang, P., and Mclean, P. J. (2015). Extracellular ATP induces intracellular alpha-synuclein accumulation via P2X1 receptormediated lysosomal dysfunction. Neurobiol. Aging 36, 1209-1220. doi: 10.1016/ j.neurobiolaging.2014.10.037

Gandelman, M., Levy, M., Cassina, P., Barbeito, L., and Beckman, J. S. (2013). P2X7 receptor-induced death of motor neurons by a peroxynitrite/FAS-dependent pathway. J. Neurochem. 126, 382-388. doi: 10.1111/jnc.12286

Gao, L., Cao, L., Qui, Y., Su, Z., Burnstock, G., Xiang, Z., et al. (2010). Blocking P2X receptors can inhibit the injury-induced proliferation of olfactory epithelium progenitor cells in adult mouse. Int. J. Pediatr. Otorhinolaryngol. 74, 747-751. doi: 10.1016/j.ijporl.2010.03.030

Gao, Y., Xu, C., Yu, K., Li, G., Wan, F., Liu, S., et al. (2010). Effect of tetramethylpyrazine on DRG neuron P2X3 receptor involved in transmitting pain after burn. Burns 36, 127-134. doi: 10.1016/j.burns.2009.04.032

Gariboldi, V., Vairo, D., Guieu, R., Marlingue, M., Ravis, E., Lagier, D., et al. (2017). Expressions of adenosine A2A receptors in coronary arteries and peripheral blood mononuclear cells are correlated in coronary artery disease patients. Int. J. Cardiol. 230, 427-431. doi: 10.1016/j.ijcard.2016.12.089

Gazzerro, E., Baldassari, S., Assereto, S., Fruscione, F., Pistorio, A., Panicucci, C., et al. (2015). Enhancement of muscle T regulatory cells and improvement of muscular dystrophic process in mdx mice by blockade of extracellular ATP/P2X axis. Am. J. Pathol. 185, 3349-3360. doi: 10.1016/j.ajpath.2015.08.010

Gehring, M. P., Kipper, F., Nicoletti, N. F., Sperotto, N. D., Zanin, R., Tamajusuku, A. S., et al. (2015). P2X7 receptor as predictor gene for glioma radiosensitivity and median survival. Int. J. Biochem. Cell Biol. 68, 92-100. doi: 10.1016/j.biocel. 2015.09.001

Gendaszewska-Darmach, E., and Kucharska, M. (2011). Nucleotide receptors as targets in the pharmacological enhancement of dermal wound healing. Purinergic Signal. 7, 193-206. doi: 10.1007/s11302-011-9233-z

Gendaszewska-Darmach, E., and Szustak, M. (2016). Thymidine 5'-Omonophosphorothioate induces HeLa cell migration by activation of the P2Y6 receptor. Purinergic Signal. 12, 199-209. doi: 10.1007/s11302-0159492-1

Gentile, D., Natale, M., Lazzerini, P. E., Capecchi, P. L., and Laghi-Pasini, F. (2015). The role of P2X7 receptors in tissue fibrosis: a brief review. Purinergic Signal. 11, 435-440. doi: 10.1007/s11302-015-9466-3
Geraghty, N. J., Watson, D., Adhikary, S. R., and Sluyter, R. (2016). P2X7 receptor in skin biology and diseases. World J. Dermatol. 5, 72-83. doi: 10.5314/wjd.v5. i2.72

Ghanbari, H., Jani, R., Hussain-Amin, A., Al-Assad, W., Huether, E., Ansari, S., et al. (2016). Role of adenosine after antral pulmonary vein isolation of paroxysmal atrial fibrillation: a randomized controlled trial. Heart Rhythm 13, 407-415. doi: 10.1016/j.hrthm.2015.10.016

Giannuzzo, A., Saccomano, M., Napp, J., Ellegaard, M., Alves, F., and Novak, I. (2016). Targeting of the P2X7 receptor in pancreatic cancer and stellate cells. Int. J. Cancer 139, 2540-2552. doi: 10.1002/ijc.30380

Gicquel, T., Le Dare, B., Boichot, E., and Lagente, V. (2017). Purinergic receptors: new targets for the treatment of gout and fibrosis. Fundam. Clin. Pharmacol. 31, 136-146. doi: 10.1111/fcp. 12256

Gidlöf, O., Sathanoori, R., Magistri, M., Faghihi, M. A., Wahlestedt, C., Olde, B., et al. (2015). Extracellular uridine triphosphate and adenosine triphosphate attenuate endothelial inflammation through miR-22-mediated ICAM-1 inhibition. J. Vasc. Res. 52, 71-80. doi: 10.1159/000431367

Gill, A., Wortham, K., Costa, D., Davis, W., Ticho, B., and Whalley, E. (2009). Protective effect of tonapofylline (BG9928), an adenosine A1 receptor antagonist, against cisplatin-induced acute kidney injury in rats. Am. J. Nephrol. 30, 521-526. doi: $10.1159 / 000248762$

Giniatullin, R., and Nistri, A. (2013). Desensitization properties of P2X3 receptors shaping pain signaling. Front. Cell Neurosci. 7:245. doi: 10.3389/fncel.2013. 00245

Giorgi, V. S., Witkin, S. S., Bannwart-Castro, C. F., Sartori, M. S., Romao-Veiga, M., Borges, V. T., et al. (2016). Elevated circulatingadenosine deaminase activity in women with preeclampsia: association with pro-inflammatory cytokine production and uric acid levels. Pregnancy Hypertens. 6, 400-405. doi: 10.1016/ j.preghy.2016.09.004

Giuliani, A. L., Colognesi, D., Ricco, T., Roncato, C., Capece, M., Amoroso, F., et al. (2014). Trophic activity of human P2X7 receptor isoforms A and B in osteosarcoma. PLOS ONE 9:e107224. doi: 10.1371/journal.pone.0107224

Giuliani, A. L., Sarti, A. C., Falzoni, S., and Di Virgilio, F. (2017). The P2X7 receptor-interleukin-1 liaison. Front. Pharmacol. 8:123. doi: 10.3389/fphar. 2017.00123

Giuliani, P., Zuccarini, M., Buccella, S., Pena-Altamira, L. E., Polazzi, E., Virgili, M., et al. (2017). Evidence for purine nucleoside phosphorylase (PNP) release from rat C6 glioma cells. J. Neurochem. 141, 208-221. doi: 10.1111/jnc.14004

Glass, R., Bardini, M., Robson, T., and Burnstock, G. (2001). Expression of nucleotide $\mathrm{P} 2 \mathrm{X}$ receptor subtypes during spermatogenesis in the adult rat testis. Cells Tissues Org. 169, 377-387. doi: 10.1159/000047905

Gloria-Bottini, F., Saccucci, P., Ammendola, M., Neri, A., Magrini, A., and Bottini, E. (2016). Genetic variability within Adenosine Deaminase gene and uterine leiomyomas. Eur. J. Obstet. Gynecol. Reprod. Biol. 199, 108-109. doi: 10.1016/j.ejogrb.2016.02.002

Gnad, T., Scheibler, S., Von Kügelgen, I., Scheele, C., Kilic, A., Glode, A., et al. (2014). Adenosine activates brown adipose tissue and recruits beige adipocytes via A2A receptors. Nature 516, 395-399. doi: 10.1038/nature13816

Go, G., Sung, J.-S., Jee, S.-C., Kim, M., Jang, W.-H., Kang, K.-Y., et al. (2017). In vitro anti-obesity effects of sesamol mediated by adenosine monophosphateactivated protein kinase and mitogen-activated protein kinase signaling in 3T3-L1 cells. Food Sci. Biotechnol. 26, 195-200. doi: 10.1007/s10068-0170026-1

Gölöncsér, F., and Sperlágh, B. (2014). Effect of genetic deletion and pharmacological antagonism of $\mathrm{P} 2 \mathrm{X} 7$ receptors in a mouse animal model of migraine. J. Headache Pain 15:24. doi: 10.1186/1129-2377-15-24

Gomez-Villafuertes, R., Garcia-Huerta, P., Diaz-Hernandez, J. I., and MirasPortugal, M. T. (2015). PI3K/Akt signaling pathway triggers P2X7 receptor expression as a pro-survival factor of neuroblastoma cells under limiting growth conditions. Sci. Rep. 5:18417. doi: 10.1038/srep18417

Gonçalves, F. M., Neis, V. B., Rieger, D. K., Lopes, M. W., Heinrich, I. A., Costa, A. P., et al. (2017). Signaling pathways underlying the antidepressant-like effect of inosine in mice. Purinergic Signal. 13, 203-214. doi: 10.1007/s11302-0169551-2

Goonetilleke, L., Ralevic, V., and Dunn, W. R. (2013). Influence of pressure on adenosine triphosphate function as a sympathetic neurotransmitter in small mesenteric arteries from the spontaneously hypertensive rat. J. Hypertens. 31, 312-320. doi: 10.1097/HJH.0b013e32835bd74d 
Gorodeski, G. I. (2009). P2X7-mediated chemoprevention of epithelial cancers. Expert. Opin. Ther. Targets 13, 1313-1332. doi: 10.1517/14728220903277249

Gorodeski, G. I. (2015). Purinergic signalling in the reproductive system. Auton. Neurosci. 191, 82-101. doi: 10.1016/j.autneu.2015.04.008

Graubardt, N., Fahrner, R., Trochsler, M., Keogh, A., Breu, K., Furer, C., et al. (2013). Promotion of liver regeneration by natural killer cells in a murine model is dependent on extracellular adenosine triphosphate phosphohydrolysis. Hepatology 57, 1969-1979. doi: 10.1002/hep.26008

Greene, S. J., Sabbah, H. N., Butler, J., Voors, A. A., Abrecht-Küpper, B. E., Düngen, H. D., et al. (2016). Partial adenosine A1 receptor agonism: a potential new therapeutic strategy for heart failure. Heart Fail. Rev. 21, 95-102. doi: 10.1007/ s10741-015-9522-7

Greer, S., Page, C. W., Joshi, T., Yan, D., Newton, R., and Giembycz, M. A. (2013). Concurrent agonism of adenosine $\mathrm{A} 2 \mathrm{~B}$ and glucocorticoid receptors in human airway epithelial cells cooperatively induces genes with anti-inflammatory potential: a novel approach to treat chronic obstructive pulmonary disease. J. Pharmacol. Exp. Ther. 346, 473-485. doi: 10.1124/jpet.113.206284

Grenz, A., Kim, J.-H., Bauerle, J. D., Tak, E., Eltzschig, H. K., and Clambey, E. T. (2012). Adora2b adenosine receptor signaling protects during acute kidney injury via inhibition of neutrophil-dependent TNF- $\alpha$ release. J. Immunol. 189, 4566-4573. doi: 10.4049/jimmunol.1201651

Greve, A. S., Skals, M., Fagerberg, S. K., Tonnus, W., Ellermann-Eriksen, S., Evans, R. J., et al. (2017). P2X1, P2X4, and P2X7 receptor knock out mice expose differential outcome of sepsis induced by $\alpha$-haemolysin producing Escherichia coli. Front. Cell Infect. Microbiol. 7:113. doi: 10.3389/fcimb.2017.00113

Gu, B. J., Field, J., Dutertre, S., Ou, A., Kilpatrick, T. J., Lechner-Scott, J., et al. (2015). A rare P2X7 variant Arg307Gln with absent pore formation function protects against neuroinflammation in multiple sclerosis. Hum. Mol. Genet. 24, 5644-5654. doi: $10.1093 / \mathrm{hmg} / \mathrm{ddv} 278$

Gualix, J., León-Otegui, M., Recuero, M., Bullido, M. J., Valdivieso, F., and MirasPortugal, M. T. (2015). Functional characterization of P2Y1 and P2X4 receptors in human neuroblastoma SK-N-MC cells. An. Real Acad. Farm. 81, 247-257.

Gubert, C., Fries, G. R., Wollenhaupt De Aguiar, B., Ribeiro, A. R., Busnello, J. V., Ribeiro, L., et al. (2013). The P2X7 purinergic receptor as a molecular target in bipolar disorder. Neuropsychiatry Neuropsychol. 8, 1-7.

Gubert, C., Jacintho Moritz, C. E., Vasconcelos-Moreno, M. P., Quadros Dos Santos, B. T., Sartori, J., Fijtman, A., et al. (2016). Peripheral adenosine levels in euthymic patients with bipolar disorder. Psychiatry Res. 246, 421-426. doi: 10.1016/j.psychres.2016.10.007

Guixà-González, R., Javanainen, M., Gómez-Soler, M., Cordobilla, B., Domingo, J. C., Sanz, F., et al. (2016). Membrane omega-3 fatty acids modulate the oligomerisation kinetics of adenosine A2A and dopamine D2 receptors. Sci. Rep. 6:19839. doi: 10.1038/srep19839

Guo, J., Fu, X., Cui, X., and Fan, M. (2015). Contributions of purinergic $\mathrm{P} 2 \mathrm{X} 3$ receptors within the midbrain periaqueductal gray to diabetes-induced neuropathic pain. J. Physiol. Sci. 65, 99-104. doi: 10.1007/s12576-0140344-5

Gupta, S., and Sharma, B. (2014). Protective effects of phosphodiesterase-1 (PDE1) and ATP sensitive potassium (KATP) channel modulators against 3-nitropropionic acid induced behavioral and biochemical toxicities in experimental Huntington $\times s$ disease. Eur. J. Pharmacol. 732, 111-122. doi: 10.1016/j.ejphar.2014.03.032

Gür, S., Kadowitz, P. J., Abdel-Mageed, A. S., Kendirci, M., Sikka, S. C., Burnstock, G., et al. (2009). Management of erectile function by penile purinergic P2 receptors in the diabetic rat. J. Urol. 181, 2375-2382. doi: 10.1016/ j.juro.2009.01.002

Gur, S., Kadowitz, P. J., and Hellstrom, W. J. (2007). Purinergic (P2) receptor control of lower genitourinary tract function and new avenues for drug action: an overview. Curr. Pharm. Des. 13, 3236-3244. doi: 10.2174/ 138161207782341277

Guzman, S. J., and Gerevich, Z. (2016). P2Y receptors in synaptic transmission and plasticity: therapeutic potential in cognitive dysfunction. Neural Plast. 2016:1207393. doi: 10.1155/2016/1207393

Guzman-Aranguez, A., Gasull, X., Diebold, Y., and Pintor, J. (2014). Purinergic receptors in ocular inflammation. Mediators Inflamm. 2014:320906. doi: 10.1155/2014/320906

Guzmán-Aránguez, A., Irazu, M., Yayon, A., and Pintor, J. (2008). P2Y receptors activated by diadenosine polyphosphates reestablish $\mathrm{Ca}^{2+}$ transients in achondroplasic chondrocytes. Bone 42, 516-523. doi: 10.1016/j.bone.2007. 10.023

Haanes, K. A., and Edvinsson, L. (2014). Expression and characterization of purinergic receptors in rat middle meningeal artery-potential role in migraine. PLOS ONE 9:e108782. doi: 10.1371/journal.pone.0108782

Hafner, S., Wagner, K., Weber, S., Gröger, M., Wepler, M., Mccook, O., et al. (2017). Role of the purinergic receptor P2XR4 after blunt chest trauma in cigarette smoke-exposed mice. Shock 47, 193-199. doi: 10.1097/SHK.0000000000000726

Hamidpour, R., Hamidpour, S., Hamidpour, M., Zarabi, M., Sohraby, M., and Shalari, M. (2016). Antipurinergic therapy with suramin as a treatment for autism spectrum disorder. J. Biomed. Sci. 5, 14. doi: 10.4172/2254-609X.100028

Hansen, R. R., Nasser, A., Falk, S., Baldvinsson, S. B., Ohlsson, P. H., Bahl, J. M., et al. (2012). Chronic administration of the selective P2X3, P2X2/3 receptor antagonist, A-317491, transiently attenuates cancer-induced bone pain in mice. Eur. J. Pharmacol. 688, 27-34. doi: 10.1016/j.ejphar.2012.05.008

Hansen, R. R., Nielsen, C. K., Nasser, A., Thomsen, S. I., Eghorn, L. F., Pham, Y., et al. (2011). P2X7 receptor-deficient mice are susceptible to bone cancer pain. Pain 152, 1766-1776. doi: 10.1016/j.pain.2011.03.024

Hansson, E., Werner, T., Björklund, U., and Skiöldebrand, E. (2016). Therapeutic innovation: inflammatory-reactive astrocytes as targets of inflammation. IBRO Rep. 1, 1-9. doi: 10.1016/j.ibror.2016.06.001

Hao, Y., Liang, J. F., Chow, A. W., Cheung, W. T., and Ko, W. H. (2014). P2Y6 receptor-mediated proinflammatory signaling in human bronchial epithelia. PLOS ONE 9:e106235. doi: 10.1371/journal.pone.0106235

Hao, Y., Zhou, G., Wu, W., Zhang, Y., Tao, L., Yao, J., et al. (2017). Synthesis and antiviral evaluation of novel N-6 substituted adenosine analogues. Tetrahedron Lett. 58, 190-193. doi: 10.1016/j.tetlet.2016.11.059

Harmse, R., Van Der Walt, M. M., Petzer, J. P., and Terre'blanche, G. (2016). Discovery of 1,3-diethyl-7-methyl-8-(phenoxymethyl)-xanthine derivatives as novel adenosine $\mathrm{A} 1$ and $\mathrm{A} 2 \mathrm{~A}$ receptor antagonists. Bioorg. Med. Chem. Lett. 26, 5951-5955. doi: 10.1016/j.bmcl.2016.10.086

Hasebe, H., Yoshida, K., Iida, M., Hatano, N., Muramatsu, T., and Aonuma, K. (2016). Right-to-left frequency gradient during atrial fibrillation initiated by right atrial ectopies and its augmentation by adenosine triphosphate: implications of right atrial fibrillation. Heart Rhythm 13, 354-363. doi: 10.1016/ j.hrthm.2015.09.030

Hattori, F., Ohshima, Y., Seki, S., Tsukimoto, M., Sato, M., Takenouchi, T., et al. (2012). Feasibility study of B16 melanoma therapy using oxidized ATP to target purinergic receptor P2X7. Eur. J. Pharmacol. 695, 20-26. doi: 10.1016/j.ejphar. 2012.09.001

Hayoz, S., Jia, C., and Hegg, C. (2012). Mechanisms of constitutive and ATP-evoked ATP release in neonatal mouse olfactory epithelium. BMC Neurosci. 13:53. doi: $10.1186 / 1471-2202-13-53$

He, W., Mazumder, A., Wilder, T., and Cronstein, B. N. (2012). Adenosine regulates bone metabolism via $A_{1}, A_{2 A}$ and $A_{2 B}$ receptors in bone marrow cells from normal humans and patients with multiple myeloma. FASEB J. 27, 3446-3454. doi: 10.1096/fj.13-231233

Headrick, J. P., Ashton, K. J., Rose'meyer, R. B., and Peart, J. N. (2013). Cardiovascular adenosine receptors: expression, actions and interactions. Pharmacol. Ther. 140, 92-111. doi: 10.1016/j.pharmthera.2013.06.002

Higgins, G., Buchanan, P., Perriere, M., Al-Alawi, M., Costello, R. W., Verriere, V., et al. (2014). Activation of P2RY11 and ATP release by lipoxin A4 restores the airway surface liquid layer and epithelial repair in cystic fibrosis. Am. J. Respir. Cell Mol. Biol. 51, 178-190. doi: 10.1165/rcmb.2012-0424OC

Ho, M. F., Low, L. M., and Rose'meyer, R. B. (2016). Pharmacology of the adenosine $\mathrm{A} 3$ receptor in the vasculature and essential hypertension. PLOS ONE 11:e0150021. doi: 10.1371/journal.pone.0150021

Ho, T., Aplin, F. P., Jobling, A. I., Phipps, J. A., De Iongh, R. U., Greferath, U., et al. (2016). Localization and possible function of P2X receptors in normal and diseased retinae. J Ocul. Pharmacol. Ther. 32, 509-517. doi: 10.1089/jop.2015. 0158

Hoegl, S., Brodsky, K. S., Blackburn, M. R., Karmouty-Quintana, H., Zwissler, B., and Eltzschig, H. K. (2015). Alveolar epithelial A2B adenosine receptors in pulmonary protection during acute lung injury. J. Immunol. 195, 1815-1824. doi: 10.4049/jimmunol.1401957

Hofer, M., Pospisil, M., Dusek, L., Hoferova, Z., Weiterova, L., and Komurkova, D. (2013). Erythropoiesis- and thrombopoiesis-characterizing parameters in adenosine A3 receptor knock-out mice. Physiol. Res. 62, 305-311. 
Hofman, P., Cherfils-Vicini, J., Bazin, M., Ilie, M., Juhel, T., Hébuterne, X., et al. (2015). Genetic and pharmacological inactivation of the purinergic P2RX7 receptor dampens inflammation but increases tumor incidence in a mouse model of colitis-associated cancer. Cancer Res. 75, 835-845. doi: 10.1158/00085472.CAN-14- 1778

Holst, S. C., Valomon, A., and Landolt, H. P. (2016). Sleep pharmacogenetics: personalized sleep-wake therapy. Annu. Rev. Pharmacol. Toxicol. 56, 577-603. doi: 10.1146/annurev-pharmtox-010715-103801

Hooper, J. S., Hadley, S. H., Morris, K. F., Breslin, J. W., Dean, J. B., and TaylorClark, T. E. (2016). Characterization of cardiovascular reflexes evoked by airway stimulation with allylisothiocyanate, capsaicin, and ATP in Sprague-Dawley rats. J. Appl. Physiol. 120, 580-591. doi: 10.1152/japplphysiol.00944.2015

Hoque, R., Sohail, M. A., Salhanick, S., Malik, A. F., Ghani, A., Robson, S. C., et al. (2012). P2X7 receptor-mediated purinergic signaling promotes liver injury in acetaminophen hepatotoxicity in mice. Am. J. Physiol. Gastrointest. Liver Physiol. 302, G1171-G1179. doi: 10.1152/ajpgi.00352.2011

Horckmans, M., Esfahani, H., Beauloye, C., Clouet, S., Di Pietrantonio, L., Robaye, B., et al. (2015). Loss of mouse P2Y4 nucleotide receptor protects against myocardial infarction through endothelin-1 downregulation. J. Immunol. 194, 1874-1881. doi: 10.4049/jimmunol.1401364

Houchi, H., Persyn, W., Legastelois, R., and Naassila, M. (2013). The adenosine A2A receptor agonist CGS 21680 decreases ethanol self-administration in both non-dependent and dependent animals. Addict. Biol. 18, 812-825. doi: 10.1111/ adb. 12032

Housley, G. D., Bringmann, A., and Reichenbach, A. (2009). Purinergic signaling in special senses. Trends Neurosci. 32, 128-141. doi: 10.1016/j.tins.2009.01.001

Howarth, A. R., Conway, B. R., and Bailey, M. A. (2015). Vascular and inflammatory actions of P2X receptors in renal injury. Auton. Neurosci. 191, 135-140. doi: 10.1016/j.autneu.2015.05.001

Hu, F., Hui, Z., Wei, W., Yang, J., Chen, Z., Guo, B., et al. (2017). Hypotonic stress promotes ATP release, reactive oxygen species production and cell proliferation via TRPV4 activation in rheumatoid arthritis rat synovial fibroblasts. Biochem. Biophys. Res. Commun. 486, 108-115. doi: 10.1016/j.bbrc.2017.03.008

Hu, H., Yang, B., Li, Y., Zhang, S., and Li, Z. (2016). Blocking of the P2X7 receptor inhibits the activation of the MMP-13 and NF- $\mathrm{BB}$ pathways in the cartilage tissue of rats with osteoarthritis. Int. J. Mol. Med. 38, 1922-1932. doi: 10.3892/ijmm.2016.2770

Hu, J., Ye, F., Cui, M., Lee, P., Wei, C., Hao, Y., et al. (2016). Protein profiling of bladder urothelial cell carcinoma. PLOS ONE 11:e0161922. doi: 10.1371/ journal.pone.0161922

Hu, S. J., Calippe, B., Lavalette, S., Roubeix, C., Montassar, F., Housset, M., et al. (2015). Upregulation of P2RX7 in Cx3cr1-deficient mononuclear phagocytes leads to increased interleukin-1 $\beta$ secretion and photoreceptor neurodegeneration. J. Neurosci. 35, 6987-6996. doi: 10.1523/JNEUROSCI. 3955-14.2015

Hu, X., Liou, A. K., Leak, R. K., Xu, M., An, C., Suenaga, J., et al. (2014). Neurobiology of microglial action in CNS injuries: receptor-mediated signaling mechanisms and functional roles. Prog. Neurobiol. 11, 60-84. doi: 10.1016/j. pneurobio.2014.06.002

Hua, X., Naselsky, W. C., Bennett, W. D., Ledent, C., Senior, B. A., and Tilley, S. L. (2013). Adenosine increases nasal mucociliary clearance rate in mice through $\mathrm{A} 2 \mathrm{~A}$ and $\mathrm{A} 2 \mathrm{~B}$ adenosine receptors. Laryngoscope 123, 306-310. doi: 10.1002/lary.23586

Huang, J., Zhang, M., Zhang, P., Liang, H., Ouyang, K., and Yang, H. T. (2016). Coupling switch of P2Y-IP3 receptors mediates differential $\mathrm{Ca}^{2+}$ signaling in human embryonic stem cells and derived cardiovascular progenitor cells. Purinergic Signal. 12, 465-478. doi: 10.1007/s11302-016-9512-9

Huang, T. C., Chang, H. Y., Hsu, C. H., Kuo, W. H., Chang, K. J., and Juan, H. F. (2008). Targeting therapy for breast carcinoma by ATP synthase inhibitor aurovertin B. J. Proteome Res. 7, 1433-1444. doi: 10.1021/pr700742h

Huang, Z. L., Liu, R., Bai, X. Y., Zhao, G., Song, J. K., Wu, S., et al. (2014). Protective effects of the novel adenosine derivative WS0701 in a mouse model of posttraumatic stress disorder. Acta Pharmacol. Sin. 35, 24-32. doi: 10.1038/ aps. 2013.143

Huber, S. M. (2012). Purinoceptor signaling in malaria-infected erythrocytes. Microbes Infect. 14, 779-786. doi: 10.1016/j.micinf.2012.04.009

Huete, F., Guzmán-Aránguez, A., Ortin, J., Hoyle, C. H., and Pintor, J. (2011). Effects of diadenosine tetraphosphate on FGF9-induced chloride flux changes in achondroplastic chondrocytes. Purinergic Signal. 7, 243-249. doi: 10.1007/ s11302-011-9234-y

Hullugundi, S. K., Ansuini, A., Ferrari, M. D., Van Den Maagdenberg, A. M., and Nistri, A. (2014). A hyperexcitability phenotype in mouse trigeminal sensory neurons expressing the R192Q Cacnala missense mutation of familial hemiplegic migraine type-1. Neuroscience 266, 244-254. doi: 10.1016/j. neuroscience.2014.02.020

Hupertan, V., Neuzillet, Y., Stücker, O., Pons, C., Leammel, E., and Lebret, T. (2012). Effects of nucleotides adenosine monophosphate and adenosine triphosphate in combination with L-arginine on male rabbit corpus cavernosum tissue. Int. J. Androl. 35, 860-866. doi: 10.1111/j.1365-2605.2012.01290.x

Hur, H., Kim, N. K., Kim, H. G., Min, B. S., Lee, K. Y., Shin, S. J., et al. (2012). Adenosine triphosphate-based chemotherapy response assay-guided chemotherapy in unresectable colorectal liver metastasis. Br. J. Cancer 106, 53-60. doi: 10.1038/bjc.2011.469

Hurtado-Alvarado, G., Domínguez-Salazar, E., Velázquez-Moctezuma, J., and Gómez-González, B. (2016). A2A adenosine receptor antagonism reverts the blood-brain barrier dysfunction induced by sleep restriction. PLOS ONE 11:e0167236. doi: 10.1371/journal.pone.0167236

Husted, L. B., Harsløf, T., Stenkjær, L., Carstens, M., Jørgensen, N. R., and Langdahl, B. L. (2013). Functional polymorphisms in the P2X7 receptor gene are associated with osteoporosis. Osteoporos. Int. 24, 949-959. doi: 10.1007/ s00198-012-2035-5

Iba, K., and Yamashita, T. (2016). "Osteoclast-mediated pain in osteoporosis," in Osteoporosis in Orthopedics: Assessment and Therapeutic Options, eds Y. Shimada and N. Miyakoshi (Tokyo: Springer), 23-34. doi: 10.1007/978-4-43155778-4_2

Ibrahim, A. S., El-Shishtawy, M. M., Zhang, W., Caldwell, R. B., and Liou, G. I. (2011). A2A adenosine receptor (A2AAR) as a therapeutic target in diabetic retinopathy. Am. J. Pathol. 178, 2136-2145. doi: 10.1016/j.ajpath.2011. 01.018

Ikeda, N., Murata, S., Maruyama, T., Tamura, T., Nozaki, R., Kawasaki, T., et al. (2012). Platelet-derived adenosine $5^{\prime}$-triphosphate suppresses activation of human hepatic stellate cell: in vitro study. Hepatol. Res. 42, 91-102. doi: 10.1111/j.1872-034X.2011.00893.x

Ikeda, Y., Zabbarova, I. V., Birder, L. A., De Groat, W. C., Mccarthy, C. J., HannaMitchell, A. T., et al. (2012). Botulinum neurotoxin serotype A suppresses neurotransmitter release from afferent as well as efferent nerves in the urinary bladder. Eur. Urol. 62, 1157-1164. doi: 10.1016/j.eururo.2012.03.031

Ilatovskaya, D. V., Palygin, O., and Staruschenko, A. (2016). Functional and therapeutic importance of purinergic signaling in polycystic kidney disease. Am. J. Physiol. Renal Physiol. 311, F1135-F1139. doi: 10.1152/ajprenal.00406. 2016

Illes, P., and Rubini, P. (2017). Regulation of neural stem/progenitor cell functions by P2X and P2Y receptors. Neural Regen. Res. 12, 395-396. doi: 10.4103/16735374.202937

Illes, P., Verkhratsky, A., Burnstock, G., and Sperlagh, B. (2016). Purines in neurodegeneration and neuroregeneration. Neuropharmacology 104, 1-3. doi: 10.1016/j.neuropharm.2016.01.020

Imarisio, C., Alchera, E., Sutti, S., Valente, G., Boccafoschi, F., Albano, E., et al. (2012). Adenosine $A_{2 A}$ receptor stimulation prevents hepatocyte lipotoxicity and non-alcoholic steatohepatitis (NASH) in rats. Clin. Sci. 123, 323-332. doi: 10.1042/CS20110504

Ingwersen, J., Wingerath, B., Graf, J., Lepka, K., Hofrichter, M., Schröter, F., et al. (2016). Dual roles of the adenosine A2a receptor in autoimmune neuroinflammation. J. Neuroinflammation 13, 48. doi: 10.1186/s12974-0160512-z

Inoue, Y., Yoshimura, K., Kurabe, N., Kahyo, T., Kawase, A., Tanahashi, M., et al. (2017). Prognostic impact of CD73 and A2A adenosine receptor expression in non-small-cell lung cancer. Oncotarget 8, 8738-8751. doi: 10.18632/oncotarget. 14434

Iriyama, T., Sun, K., Parchim, N. F., Li, J., Zhao, C., Song, A., et al. (2015). Elevated placental adenosine signaling contributes to the pathogenesis of preeclampsia. Circulation 131, 730-741. doi: 10.1161/CIRCULATIONAHA.114. 013740

Ishikawa, T., Miyagi, M., Ohtori, S., Aoki, Y., Ozawa, T., Doya, H., et al. (2005). Characteristics of sensory DRG neurons innervating the lumbar facet joints in rats. Eur. Spine J. 14, 559-564. doi: 10.1007/s00586-004-0834-x 
Ishimaru, M., Tsukimoto, M., Harada, H., and Kojima, S. (2013). Involvement of P2Y11 receptor in IFN- $\gamma$-induced IL- 6 production in human keratinocytes. Eur. J. Pharmacol. 703, 67-73. doi: 10.1016/j.ejphar.2013.02.020

Iskandarov, E., Kadaba Srinivasan, P., Xin, W., Bleilevens, C., Afify, M., Hamza, A., et al. (2016). Protective effects of adenosine receptor agonist in a cirrhotic liver resection model. Hepat. Mon. 16:e36821. doi: 10.5812/hepatmon.36821

Iwata, M., Ota, K. T., Li, X. Y., Sakaue, F., Li, N., Dutheil, S., et al. (2016). Psychological stress activates the inflammasome via release of adenosine triphosphate and stimulation of the purinergic type $2 \mathrm{X} 7$ receptor. Biol. Psychiatry 80, 12-22. doi: 10.1016/j.biopsych.2015.11.026

Jacob, F., Novo, C. P., Bachert, C., and Crombruggen, K. (2013). Purinergic signaling in inflammatory cells: P2 receptor expression, functional effects, and modulation of inflammatory responses. Purinergic Signal. 9, 285-306. doi: 10.1007/s11302-013-9357-4

Jacobs, B., and Dussor, G. (2016). Neurovascular contributions to migraine: moving beyond vasodilation. Neuroscience 338, 130-144. doi: 10.1016/j.neuroscience. 2016.06.012

Jacobson, K. A., and Muller, C. E. (2016). Medicinal chemistry of adenosine, P2Y and P2X receptors. Neuropharmacology 104, 31-49. doi: 10.1016/j.neuropharm. 2015.12.001

Jajoo, S., Mukherjea, D., Watabe, K., and Ramkumar, V. (2009). Adenosine A3 receptor suppresses prostate cancer metastasis by inhibiting NADPH oxidase activity. Neoplasia 11, 1132-1145. doi: 10.1593/neo.09744

Jalkanen, J., Yegutkin, G. G., Hollmén, M., Aalto, K., Kiviniemi, T., Salomaa, V., et al. (2015). Aberrant circulating levels of purinergic signaling markers are associated with several key aspects of peripheral atherosclerosis and thrombosis. Circ. Res. 116, 1206-1215. doi: 10.1161/CIRCRESAHA.116.305715

Jameel, M. N., Xiong, Q., Mansoor, A., Bache, R. J., and Zhang, J. (2016). ATP sensitive $\mathrm{K}^{+}$channels are critical for maintaining myocardial perfusion and high energy phosphates in the failing heart. J. Mol. Cell. Cardiol. 92, 116-121. doi: 10.1016/j.yjmcc.2016.02.005

Janes, K., Symons-Liguori, A. M., Jacobson, K. A., and Salvemini, D. (2016). Identification of A3 adenosine receptor agonists as novel non-narcotic analgesics. Br. J. Pharmacol. 173, 1253-1267. doi: 10.1111/bph.13446

Jared Bunch, T. (2015). One drug, two outcomes after atrial fibrillation ablation: the situational irony of adenosine. Eur. Heart J. 36, 3288-3290. doi: 10.1093/ eurheartj/ehv473

Jastrzebska, J., Nowak, E., Smaga, I., Bystrowska, B., Frankowska, M., Bader, M., et al. (2014). Adenosine A2A receptor modulation of nicotine-induced locomotor sensitization. A pharmacological and transgenic approach. Neuropharmacology 81, 318-326. doi: 10.1016/j.neuropharm.2014.03.002

Jelassi, B., Anchelin, M., Chamouton, J., Cayuela, M. L., Clarysse, L., Li, J., et al. (2013). Anthraquinone emodin inhibits human cancer cell invasiveness by antagonizing P2X7 receptors. Carcinogenesis 34, 1487-1496. doi: 10.1093/ carcin/bgt099

Jenner, P. (2014). An overview of adenosine A2A receptor antagonists in Parkinson's disease. Int. Rev. Neurobiol. 119, 71-86. doi: 10.1016/B978-0-12801022-8.00003-9

Jeong, J. Y., Kim, J., Kim, B., Kim, J., Shin, Y., Kim, J., et al. (2016). IL-1ra secreted by ATP-induced P2Y2 negatively regulates MUC5AC overproduction via PLCß3 during airway inflammation. Mediators Inflamm. 2016:7984853. doi: 10.1155/2016/7984853

Ji, X., Naito, Y., Hirokawa, G., Weng, H., Hiura, Y., Takahashi, R., et al. (2012). P2X7 receptor antagonism attenuates the hypertension and renal injury in Dahl salt-sensitive rats. Hypertens. Res. 35, 173-179. doi: 10.1038/hr.2011.153

Jia, C., Sangsiri, S., Belock, B., Iqbal, T. R., Pestka, J. J., and Hegg, C. C. (2011). ATP mediates neuroprotective and neuroproliferative effects in mouse olfactory epithelium following exposure to satratoxin $\mathrm{G}$ in vitro and in vivo. Toxicol. Sci. 124, 169-178. doi: 10.1093/toxsci/kfr213

Jia, X., Naito, H., Yetti, H., Tamada, H., Kitamori, K., Hayashi, Y., et al. (2012). The modulation of hepatic adenosine triphosphate and inflammation by eicosapentaenoic acid during severe fibrotic progression in the SHRSP5/Dmcr rat model. Life Sci. 90, 934-943. doi: 10.1016/j.lfs.2012.04.029

Jian, R., Sun, Y., Wang, Y., Yu, J., Zhong, L., and Zhou, P. (2012). CD73 protects kidney from ischemia-reperfusion injury through reduction of free radicals. APMIS 120, 130-138. doi: 10.1111/j.1600-0463.2011.02827.x

Jiang, S., Zhang, Y., Zheng, J. H., Li, X., Yao, Y. L., Wu, Y. L., et al. (2017). Potentiation of hepatic stellate cell activation by extracellular ATP is dependent on P2X7R-mediated NLRP3 inflammasome activation. Pharmacol. Res. 117, 82-93. doi: 10.1016/j.phrs.2016.11.040

Jiménez, M., Clavé, P., Accarino, A., and Gallego, D. (2014). Purinergic neuromuscular transmission in the gastrointestinal tract; functional basis for future clinical and pharmacological studies. Br. J. Pharmacol. 171, 4360-4375. doi: $10.1111 /$ bph. 12802

Jiménez, M., De Diego, M., Martínez-Cutillas, M., Mañé Reed, N., Gallego, D., Ojanguren, M., et al. (2015). Purinergic and nitrergic inhibitory neuromuscular transmission in ganglionic, transitional and aganglionic segments from Hirschsprung's disease patients. Neurogastroenterol. Motil. 27, 71.

Jimenez-Mateos, E. M., Arribas-Blazquez, M., Sanz-Rodriguez, A., Concannon, C., Olivos-Ore, L. A., Reschke, C. R., et al. (2015). microRNA targeting of the P2X7 purinoceptor opposes a contralateral epileptogenic focus in the hippocampus. Sci. Rep. 5:17486. doi: 10.1038/srep 17486

Jin, F. (2016). Analysis on mechanism of ATP-sensitive $\mathrm{K}^{+}$channel opener natakalim improving congestive heart failure after myocardial infarction. Exp. Ther. Med. 12, 3993-3997. doi: 10.3892/etm.2016.3853

Jin, H., Seo, J., Eun, S. Y., Joo, Y. N., Park, S. W., Lee, J. H., et al. (2014). P2Y2R activation by nucleotides promotes skin wound-healing process. Exp. Dermatol. 23, 480-485. doi: 10.1111/exd.12440

Jin, W., Xu, W., Chen, J., Zhang, X., Shi, L., and Ren, C. (2016). Adenosine kinase facilitated astrogliosis-induced cortical neuronal death in traumatic brain injury. J. Mol. Histol. 47, 259-271. doi: 10.1007/s10735-016-9670-7

Jörg, M., Scammells, P. J., and Capuano, B. (2014). The dopamine D2 and adenosine A2A receptors: past, present and future trends for the treatment of Parkinson's disease. Curr. Med. Chem. 21, 3188-3210. doi: 10.2174/ 1389200215666140217110716

Jørgensen, N. R., Boeynaems, J. M., and Di Virgilio, F. (2011). European meeting "P2 receptors: new targets for the treatment of osteoporosis". Purinergic Signal. 7, 275-276. doi: 10.1007/s11302-011-9223-1

Jørgensen, N. R., Grove, E. L., Schwarz, P., and Vestergaard, P. (2012). Clopidogrel and the risk of osteoporotic fractures: a nationwide cohort study. J. Intern. Med. 272, 385-393. doi: 10.1111/j.1365-2796.2012.02535.x

Jørgensen, N. R., Syberg, S., and Ellegaard, M. (2015). The role of P2X receptors in bone biology. Curr. Med. Chem. 22, 902-914. doi: 10.2174/ 0929867321666141215094749

Joseph, E. K., Green, P. G., Ferrari, L. F., and Levine, J. D. (2015). Homocysteineinduced attenuation of vascular endothelium-dependent hyperalgesia in the rat. Neuroscience 284, 678-684. doi: 10.1016/j.neuroscience.2014.10.056

Junger, W. G. (2011). Immune cell regulation by autocrine purinergic signalling. Nat. Rev. Immunol. 11, 201-212. doi: 10.1038/nri2938

Kaan, T. K., Yip, P. K., Patel, S., Davies, M., Marchand, F., Cockayne, D. A., et al. (2010). Systemic blockade of P2X 3 and P2X2/3 receptors attenuates bone cancer pain behaviour in rats. Brain 133, 2549-2564. doi: 10.1093/brain/awq194

Kaiser, M., Penk, A., Franke, H., Krügel, U., Norenberg, W., Huster, D., et al. (2016). Lack of functional P2X7 receptor aggravates brain edema development after middle cerebral artery occlusion. Purinergic Signal. 12, 453-463. doi: 10.1007/ s11302-016-9511-x

Kao, Y. H., Lin, M. S., Chen, C. M., Wu, Y. R., Chen, H. M., Lai, H. L., et al. (2017). Targeting ENT1 and adenosine tone for the treatment of Huntington's disease. Hum. Mol. Genet. 26, 467-478. doi: 10.1093/hmg/ddw402

Kapila, N., Higa, J. T., Longhi, M. S., and Robson, S. C. (2013). Autoimmune hepatitis: clinical review with insights into the purinergic mechanism of disease. J. Clin. Transl. Hepatol. 1, 79-86. doi: 10.14218/JCTH.2013.00015

Karmakar, M., Katsnelson, M. A., Dubyak, G. R., and Pearlman, E. (2016). Neutrophil P2X7 receptors mediate NLRP3 inflammasome-dependent IL-1 $\beta$ secretion in response to ATP. Nat. Commun. 7:10555. doi: 10.1038/ ncomms 10555

Kaster, M. P., Machado, N. J., Silva, H. B., Nunes, A., Ardais, A. P., Santana, M., et al. (2015). Caffeine acts through neuronal adenosine A2A receptors to prevent mood and memory dysfunction triggered by chronic stress. Proc. Natl. Acad. Sci. U.S.A 112, 7833-7838. doi: 10.1073/pnas.1423088112

Katz, N. K., Ryals, J. M., and Wright, D. E. (2015). Central or peripheral delivery of an adenosine A1 receptor agonist improves mechanical allodynia in a mouse model of painful diabetic neuropathy. Neuroscience 285, 312-323. doi: 10.1016/ j.neuroscience.2014.10.065

Kavanagh, K. A., Schreiner, D. C., Levis, S. C., O'neill, C. E., and Bachtell, R. K. (2015). Role of adenosine receptor subtypes in methamphetamine reward and 
reinforcement. Neuropharmacology 89, 265-273. doi: 10.1016/j.neuropharm. 2014.09.030

Kawai, Y., Kaidoh, M., and Ohhashi, T. (2008). MDA-MB-231 produces ATPmediated ICAM-1-dependent facilitation of the attachment of carcinoma cells to human lymphatic endothelial cells. Am. J. Physiol. Cell Physiol. 295, C1123-C1132. doi: 10.1152/ajpcell.00247.2008

Kawamura, H., Aswad, F., Minagawa, M., Govindarajan, S., and Dennert, G. (2006). P2X7 receptors regulate NKT cells in autoimmune hepatitis. J. Immunol. 176, 2152-2160. doi: 10.4049/jimmunol.176.4.2152

Ke, J., Yao, B., Li, T., Cui, S., and Ding, H. (2015). A2 adenosine receptor-mediated cardioprotection against reperfusion injury in rat hearts is associated with autophagy downregulation. J. Cardiovasc. Pharmacol. 66, 25-34. doi: 10.1097/ FJC.0000000000000239

Keay, S. K., Birder, L. A., and Chai, T. C. (2014). Evidence for bladder urothelial pathophysiology in functional bladder disorders. Biomed Res. Int. 2014:865463. doi: $10.1155 / 2014 / 865463$

Kehasse, A., Rich, C. B., Lee, A., Mccomb, M. E., Costello, C. E., and TrinkausRandall, V. (2013). Epithelial wounds induce differential phosphorylation changes in response to purinergic and EGF receptor activation. Am. J. Pathol. 183, 1841-1852. doi: 10.1016/j.ajpath.2013.08.015

Keystone, E. C., Wang, M. M., Layton, M., Hollis, S., and Mcinnes, I. B. (2012). Clinical evaluation of the efficacy of the $\mathrm{P} 2 \mathrm{X} 7$ purinergic receptor antagonist AZD9056 on the signs and symptoms of rheumatoid arthritis in patients with active disease despite treatment with methotrexate or sulphasalazine. Ann. Rheum. Dis. 71, 1630-1635. doi: 10.1136/annrheumdis-2011-143578

Khalid, M., Brisson, L., Tariq, M., Hao, Y., Guibon, R., Fromont, G., et al. (2017). Carcinoma-specific expression of P2Y11 receptor and its contribution in ATPinduced purinergic signalling and cell migration in human hepatocellular carcinoma cells. Oncotarget 8, 37278-37290. doi: 10.18632/oncotarget.16191

Khoja, S., Shah, V., Garcia, D., Asatryan, L., Jakowec, M. W., and Davies, D. L. (2016). Role of purinergic P2X4 receptors in regulating striatal dopamine homeostasis and dependent behaviors. J. Neurochem. 139, 134-148. doi: 10.1111/jnc. 13734

Kilinc, E., Koroleva, K., Guerrero Toro, C., Töre, F., and Giniatullin, R. (2015). The role of adenosine triphosphate and its receptors in migraine pathophysiology. Acta Physiol. 215, 44.

Killeen, M. E., Ferris, L., Kupetsky, E. A., Falo, L. Jr., and Mathers, A. R. (2013). Signaling through purinergic receptors for ATP induces human cutaneous innate and adaptive Th17 responses: implications in the pathogenesis of psoriasis. J. Immunol. 190, 4324-4336. doi: 10.4049/jimmunol.1202045

Kim, H., Walsh, M. C., Takegahara, N., Middleton, S. A., Shin, H. I., Kim, J., et al. (2017). The purinergic receptor P2X5 regulates inflammasome activity and hyper-multinucleation of murine osteoclasts. Sci. Rep. 7, 196. doi: 10.1038/ s41598-017-00139-2

Kim, M. J., Turner, C. M., Hewitt, R., Smith, J., Bhangal, G., Pusey, C. D., et al. (2014). Exaggerated renal fibrosis in P2X4 receptor-deficient mice following unilateral ureteric obstruction. Nephrol. Dial. Transplant. 29, 1350-1361. doi: 10.1093/ndt/gfu019

Kimbler, D. E., Shields, J., Yanasak, N., Vender, J. R., and Dhandapani, K. M. (2012). Activation of P2X7 promotes cerebral edema and neurological injury after traumatic brain injury in mice. PLOS ONE 7:e41229. doi: 10.1371/journal. pone.0041229

Kinnamon, S. C., and Finger, T. E. (2013). A taste for ATP: neurotransmission in taste buds. Front. Cell Neurosci. 7:264. doi: 10.3389/fncel.2013.00264

Kira, S., Yoshiyama, M., Tsuchiya, S., Shigetomi, E., Miyamoto, T., Nakagomi, H., et al. (2017). P2Y6-deficiency increases micturition frequency and attenuates sustained contractility of the urinary bladder in mice. Sci. Rep. 7, 771. doi: 10.1038/s41598-017-00824-2

Kishore, B. K., Carlson, N. G., Ecelbarger, C. M., Kohan, D. E., Müller, C. E., Nelson, R. D., et al. (2015). Targeting renal purinergic signalling for the treatment of lithium-induced nephrogenic diabetes insipidus. Acta Physiol. 214, 176-188. doi: 10.1111/apha.12507

Kitta, T., Chancellor, M. B., De Groat, W. C., Kuno, S., Nonomura, K., and Yoshimura, N. (2012). Suppression of bladder overactivity by adenosine A2A receptor antagonist in a rat model of Parkinson disease. J. Urol. 187, 1890-1897. doi: 10.1016/j.juro.2011.12.062

Kizilirmak, F., Gunes, H. M., Demir, G. G., Gokdeniz, T., Guler, E., Cakal, B., et al. (2015). Impact of intracoronary adenosine on myonecrosis in patients with unstable angina pectoris undergoing percutaneous coronary intervention. Cardiovasc. Drugs Ther. 29, 519-526. doi: 10.1007/s10557-015-6631-4

Knight, G. E., Bodin, P., De Groat, W. C., and Burnstock, G. (2002). ATP is released from guinea pig ureter epithelium on distension. Am. J. Physiol. Renal Physiol. 282, F281-F288. doi: 10.1152/ajprenal.00293.2000

Kofoed, K., Skov, L., and Zachariae, C. (2015). New drugs and treatment targets in psoriasis. Acta Derm. Venereol. 95, 133-139. doi: 10.2340/00015555-1931

Köhler, D., Streienberger, A., Morote-García, J. C., Granja, T. F., Schneider, M., Straub, A., et al. (2016). Inhibition of adenosine kinase attenuates acute lung injury. Crit. Care Med. 44, e181-e189. doi: 10.1097/CCM.0000000000001370

Komai, A. M., Brännmark, C., Musovic, S., and Olofsson, C. S. (2014). PKAindependent CAMP stimulation of white adipocyte exocytosis and adipokine secretion: modulations by $\mathrm{Ca}^{2+}$ and ATP. J. Physiol. 592, 5169-5186. doi: 10.1113/jphysiol.2014.280388

Kornum, B. R., Kawashima, M., Faraco, J., Lin, L., Rico, T. J., Hesselson, S., et al. (2011). Common variants in P2RY11 are associated with narcolepsy. Nat. Genet. 43, 66-71. doi: 10.1038/ng.734

Kowaluk, E. A., and Jarvis, M. F. (2000). Therapeutic potential of adenosine kinase inhibitors. Expert Opin. Investig. Drugs 9, 551-564. doi: 10.1517/13543784.9. 3.551

Krasowska, E., Róg, J., Sinadinos, A., Young, C. N., Górecki, D. C., and Zablocki, K. (2014). Purinergic receptors in skeletal muscles in health and in muscular dystrophy. Postepy Biochem. 60, 483-489.

Kretschmar, C., Oyarzún, C., Villablanca, C., Jaramillo, C., Alarcón, S., Perez, G., et al. (2016). Reduced adenosine uptake and its contribution to signaling that mediates profibrotic activation in renal tubular epithelial cells: implication in diabetic nephropathy. PLOS ONE 11:e0147430. doi: 10.1371/journal.pone. 0147430

Krizaj, D., Ryskamp, D. A., Tian, N., Tezel, G., Mitchell, C. H., Slepak, V. Z., et al. (2014). From mechanosensitivity to inflammatory responses: new players in the pathology of glaucoma. Curr. Eye Res. 39, 105-119. doi: 10.3109/02713683.2013. 836541

Krügel, U. (2016). Purinergic receptors in psychiatric disorders. Neuropharmacology 104, 212-225. doi: 10.1016/j.neuropharm.2015.10.032

Krügel, U., Köles, L., and Illes, P. (2013). Integration of neuronal and glial signalling by pyramidal cells of the rat prefrontal cortex; control of cognitive functions and addictive behaviour by purinergic mechanisms. Neuropsychopharmacol. Hung. $15,206-213$.

Kruglov, E. A., Correa, P. R., Arora, G., Yu, J., Nathanson, M. H., and Dranoff, J. A. (2007). Molecular basis for calcium signaling in hepatic stellate cells. Am. J. Physiol. Gastrointest. Liver Physiol. 292, G975-G982.

Kuan, Y. H., Shih, H. C., Tang, S. C., Jeng, J. S., and Shyu, B. C. (2015). Targeting P2X7 receptor for the treatment of central post-stroke pain in a rodent model. Neurobiol. Dis. 78, 134-145. doi: 10.1016/j.nbd.2015.02.028

Kuan, Y. H., and Shyu, B. C. (2016). Nociceptive transmission and modulation via P2X receptors in central pain syndrome. Mol. Brain 9, 58. doi: 10.1186/s13041016-0240-4

Kudira, R., Malinka, T., Kohler, A., Dosch, M., De Aguero, M. G., Melin, N., et al. (2016). P2X1-regulated IL-22 secretion by innate lymphoid cells is required for efficient liver regeneration. Hepatology 63, 2004-2017. doi: 10.1002/hep.28492

Kumahashi, N., Naitou, K., Nishi, H., Oae, K., Watanabe, Y., Kuwata, S., et al. (2011). Correlation of changes in pain intensity with synovial fluid adenosine triphosphate levels after treatment of patients with osteoarthritis of the knee with high-molecular-weight hyaluronic acid. Knee 18, 160-164. doi: 10.1016/j. knee.2010.04.013

Kumar, V., Chapple, C. R., Rosario, D., Tophill, P. R., and Chess-Williams, R. (2010). In vitro release of adenosine triphosphate from the urothelium of human bladders with detrusor overactivity, both neurogenic and idiopathic. Eur. Urol. 57, 1087-1092. doi: 10.1016/j.eururo.2009.11.042

Kurashima, Y., and Kiyono, H. (2016). "Physiological and pathological inflammation at the mucosal frontline," in Chronic Inflammation: Mechanisms and Regulation, eds M. Miyasaka and K. Takatsu (Tokyo: Springer), 567-590.

Kurashima, Y., Kiyono, H., and Kunisawa, J. (2015). Pathophysiological role of extracellular purinergic mediators in the control of intestinal inflammation. Mediators Inflamm. 2015:427125. doi: 10.1155/2015/427125

Kurlak, L. O., Williams, P. J., Bulmer, J. N., Broughton Pipkin, F., and Mistry, H. D. (2015). Placental expression of adenosine A2A receptor and hypoxia inducible factor-1 alpha in early pregnancy, term and pre-eclamptic pregnancies: 
interactions with placental renin-angiotensin system. Placenta 36, 611-613. doi: $10.1016 /$ j.placenta.2015.02.011

Kvist, T. M., Schwarz, P., and Jørgensen, N. R. (2014). The P2X7 receptor: a key player in immune-mediated bone loss? Sci. World J. 2014:954530. doi: 10.1155/ 2014/954530

Kwon, S. G., Roh, D. H., Yoon, S. Y., Moon, J. Y., Choi, S. R., Choi, H. S., et al. (2014). Blockade of peripheral P2Y1 receptors prevents the induction of thermal hyperalgesia via modulation of TRPV1 expression in carrageenaninduced inflammatory pain rats: involvement of p38 MAPK phosphorylation in DRGs. Neuropharmacology 79, 368-379. doi: 10.1016/j.neuropharm.2013. 12.005

Kyle, B. D. (2014). Ion channels of the mammalian urethra. Channels 8, 393-401. doi: $10.4161 / 19336950.2014 .954224$

Labazi, H., Teng, B., Zhou, Z., and Mustafa, S. J. (2016). Enhanced A2A adenosine receptor-mediated increase in coronary flow in type I diabetic mice. J. Mol. Cell. Cardiol. 90, 30-37. doi: 10.1016/j.yjmcc.2015.11.033

Lahmann, C., Clark, R. H., Iberl, M., and Ashcroft, F. M. (2014). A mutation causing increased KATP channel activity leads to reduced anxiety in mice. Physiol. Behav. 129, 79-84. doi: 10.1016/j.physbeh.2014.02.031

Lajdova, I., Oksa, A., Chorvat, J. D., Topor, P., and Spustova, V. (2012). Purinergic $\mathrm{P} 2 \mathrm{X} 7$ receptors participate in disturbed intracellular calcium homeostasis in peripheral blood mononuclear cells of patients with chronic kidney disease. Kidney Blood Press. Res. 35, 48-57. doi: 10.1159/000330349

Laketa, D., Savic, J., Bjelobaba, I., Lavrnja, I., Vasic, V., Stojiljkovic, M., et al. (2015). Brain injury alters ectonucleotidase activities and adenine nucleotide levels in rat serum. J. Med. Biochem. 34, 215-222. doi: 10.2478/jomb-2014-0025

Lau, O. C., Samarawickrama, C., and Skalicky, S. E. (2014). P2Y2 receptor agonists for the treatment of dry eye disease: a review. Clin. Ophthalmol. 8, 327-334. doi: 10.2147/OPTH.S39699

Lavrnja, I., Laketa, D., Savic, D., Bozic, I., Bjelobaba, I., Pekovic, S., et al. (2015). Expression of a second ecto- $5^{\prime}$-nucleotidase variant besides the usual protein in symptomatic phase of experimental autoimmune encephalomyelitis. J. Mol. Neurosci. 55, 898-911. doi: 10.1007/s12031-014-0445-x

Layland, J., Carrick, D., Lee, M., Oldroyd, K., and Berry, C. (2014). Adenosine: physiology, pharmacology, and clinical applications. JACC Cardiovasc. Interv. 7, 581-591. doi: 10.1016/j.jcin.2014.02.009

Ledderose, C., Bao, Y., Kondo, Y., Fakhari, M., Slubowski, C., Zhang, J., et al. (2016). Purinergic signaling and the immune response in sepsis: a review. Clin. Ther. 38, 1054-1065. doi: 10.1016/j.clinthera.2016.04.002

Lee, B. H., Hwang, D. M., Palaniyar, N., Grinstein, S., Philpott, D. J., and $\mathrm{Hu}$, J. (2012). Activation of P2X7 receptor by ATP plays an important role in regulating inflammatory responses during acute viral infection. PLOS ONE 7:e35812. doi: 10.1371/journal.pone.0035812

Lee, C. F., and Chern, Y. (2014). Adenosine receptors and Huntington's disease. Int. Rev. Neurobiol. 119, 195-232. doi: 10.1016/B978-0-12-801022-8.00010-6

Lee, C. Y. (2015). Adenosine protects Sprague Dawley rats from high-fat diet and repeated acute restraint stress-induced intestinal inflammation and altered expression of nutrient transporters. J. Anim. Physiol. Anim. Nutr. 99, 317-325. doi: 10.1111/jpn.12247

Lee, D., Myers, J. S., and Katz, L. J. (2016). The role of adenosine receptor agonists in ocular disorders. Drugs Future 41, 547-552. doi: 10.1358/dof.2016.041.09. 2533858

Lemaire, A., Vanorlé, M., Horckmans, M., Di Pietrantonio, L., Clouet, S., Robaye, B., et al. (2016). Mouse P2Y4 nucleotide receptor is a negative regulator of cardiac adipose-derived stem cell differentiation and cardiac fat formation. Stem Cells Dev. 26, 363-373. doi: 10.1089/scd.2016.0166

Lerman, B. B. (2015). Ventricular tachycardia: mechanistic insights derived from adenosine. Circ. Arrhythm. Electrophysiol. 8, 483-491. doi: 10.1161/CIRCEP. 115.001693

Lertsuwan, K., Peters, W., Johnson, L., Lertsuwan, J., Marwa, I., and Sikes, R. A. (2017). Purinergic receptor expression and cellular responses to purinergic agonists in human prostate cancer cells. Anticancer Res. 37, 529-537. doi: 10.21873/anticanres.11345

Leyva-Grado, V. H., Ermler, M. E., Schotsaert, M., Gonzalez, M. G., Gillespie, V., Lim, J. K., et al. (2017). Contribution of the purinergic receptor P2X7 to development of lung immunopathology during influenza virus infection. mBio 8:e00229-17. doi: 10.1128/mBio.00229-17
Li, C. L., Yang, X. L., Wang, J. J., Du, G. H., Yang, W. M., and Zhang, H. P. (2015). Effects of intracavernous injection of P2X3 and NK1 receptor antagonists on erectile dysfunction induced by spinal cord transection in rats. Andrologia 47, 25-29. doi: 10.1111/and.12217

Li, H., Chen, O., Ye, Z., Zhang, R., Hu, H., Zhang, N., et al. (2017). Inhalation of high concentrations of hydrogen ameliorates liver ischemia/reperfusion injury through A2A receptor mediated PI3K-Akt pathway. Biochem. Pharmacol. 130, 83-92. doi: 10.1016/j.bcp.2017.02.003

Li, H. J., Wang, L. Y., Qu, H. N., Yu, L. H., Burnstock, G., Ni, X., et al. (2011). P2Y2 receptor-mediated modulation of estrogen-induced proliferation of breast cancer cells. Mol. Cell. Endocrinol. 338, 28-37. doi: 10.1016/j.mce.2011. 02.014

Li, L., Huang, L., Ye, H., Song, S. P., Bajwa, A., Lee, S. J., et al. (2012). Dendritic cells tolerized with adenosine A2AR agonist attenuate acute kidney injury. J. Clin. Invest. 122, 3931-3942. doi: 10.1172/JCI63170

Li, S., Geiger, N. H., Soliman, M. L., Hui, L., Geiger, J. D., and Chen, X. (2015). Caffeine, through adenosine A3 receptor-mediated actions, suppresses amyloid- $\beta$ protein precursor internalization and amyloid- $\beta$ generation. J. Alzheimers Dis. 47, 73-83. doi: 10.3233/JAD-142223

Li, W., Li, Y., Li, G., Zhou, Z., Chang, X., Xia, Y., et al. (2017). Ectopic expression of the ATP synthase beta subunit on the membrane of PC-3M cells supports its potential role in prostate cancer metastasis. Int. J. Oncol. 50, 1312-1320. doi: 10.3892/ijo.2017.3878

Li, W., Silva, H. B., Real, J., Wang, Y. M., Rial, D., Li, P., et al. (2015). Inactivation of adenosine A2A receptors reverses working memory deficits at early stages of Huntington's disease models. Neurobiol. Dis. 79, 70-80. doi: 10.1016/j.nbd. 2015.03.030

Li, X., Qi, X., Zhou, L., Catera, D., Rote, N. S., Potashkin, J., et al. (2007). Decreased expression of P2X7 in endometrial epithelial pre-cancerous and cancer cells. Gynecol. Oncol. 106, 233-243. doi: 10.1016/j.ygyno.2007.03.032

Li, X., Qi, X., Zhou, L., Fu, W., Bdul-Karim, F. W., Maclennan, G., et al. (2009). $\mathrm{P} 2 \mathrm{X} 7$ receptor expression is decreased in epithelial cancer cells of ectodermal, uro-genital sinus, and distal paramesonephric duct origin. Purinergic Signal. 5, 351-368. doi: 10.1007/s11302-009-9161-3

Li, Y., He, Y., Chen, M., Pu, Z., Chen, L., Li, P., et al. (2016). Optogenetic activation of adenosine A2A receptor signaling in the dorsomedial striatopallidal neurons suppresses goal-directed behavior. Neuropsychopharmacology 41, 1003-1013. doi: 10.1038/npp.2015.227

Li, Y., Xue, L., Miao, Q., Mao, F., Yao, L., Yuan, J., et al. (2013). Expression and electrophysiological characteristics of $\mathrm{P} 2 \mathrm{X} 3$ receptors in interstitial cells of Cajal in rats with partial bladder outlet obstruction. BJU Int. 111, 843-851. doi: 10.1111/j.1464-410X.2012.11408.x

Lietsche, J., Imran, I., and Klein, J. (2016). Extracellular levels of ATP and acetylcholine during lithium-pilocarpine induced status epilepticus in rats. Neurosci. Lett. 611, 69-73. doi: 10.1016/j.neulet.2015.11.028

Liñán-Rico, A., Wunderlich, J. E., Enneking, J. T., Tso, D. R., Grants, I., Williams, K. C., et al. (2015). Neuropharmacology of purinergic receptors in human submucous plexus: involvement of P2X1, P2X2, P2X3 channels, P2Y and A3 metabotropic receptors in neurotransmission. Neuropharmacology 95, 83-99. doi: 10.1016/j.neuropharm.2015.02.014

Lindberg, D., Shan, D., Ayers-Ringler, J., Oliveros, A., Benitez, J., Prieto, M., et al. (2015). Purinergic signaling and energy homeostasis in psychiatric disorders. Curr. Mol. Med. 15, 275-295. doi: 10.2174/156652401566615033016 3724

Lioi, A. B., Ferrari, B. M., Dubyak, G. R., Weinberg, A., and Sieg, S. F. (2015). Human $\beta$ defensin-3 increases CD86 expression on monocytes by activating the ATP-gated channel P2X7. J. Immunol. 195, 4438-4445. doi: 10.4049/jimmunol. 1401319

Liu, F., Tantry, U. S., and Gurbel, P. A. (2015). P2Y12 receptor inhibitors for secondary prevention of ischemic stroke. Expert Opin. Pharmacother. 16, 1149-1165. doi: 10.1517/14656566.2015.1035256

Liu, G., Li, M., and Daneshgari, F. (2008). Temporal expression of muscarinic and purinergic receptors in diabetic rat bladder. Neurourol. Urodyn. 27, 594-595.

Liu, H., Jiang, Y., and Kuo, H. (2013). Increased suburothelial nerve fiber and purinergic P2X3 receptor expressions in patients with idiopathic detrusor overactivity and their relationship with botulinum toxin A therapeutic outcome. Neurourol. Urodyn. 32, 733-735. 
Liu, H., and Xia, Y. (2015). Beneficial and detrimental role of adenosine signaling in diseases and therapy. J. Appl. Physiol. 119, 1173-1182. doi: 10.1152/ japplphysiol.00350.2015

Liu, J., Prell, T., Stubendorff, B., Keiner, S., Ringer, T., Gunkel, A., et al. (2016). Down-regulation of purinergic P2X7 receptor expression and intracellular calcium dysregulation in peripheral blood mononuclear cells of patients with amyotrophic lateral sclerosis. Neurosci. Lett. 630, 77-83. doi: 10.1016/j.neulet. 2016.07.039

Liu, K., Vergani, A., Zhao, P., Ben, N. M., Wu, X., Iken, K., et al. (2014). Inhibition of the purinergic pathway prolongs mouse lung allograft survival. Am. J. Respir. Cell Mol. Biol. 51, 300-310. doi: 10.1165/rcmb.2013-0362OC

Liu, M., Yang, H., Fang, D., Yang, J. J., Cai, J., Wan, Y., et al. (2013). Upregulation of P2X3 receptors by neuronal calcium sensor protein VILIP-1 in dorsal root ganglions contributes to the bone cancer pain in rats. Pain 154, 1551-1568. doi: $10.1016 /$ j.pain.2013.04.022

Liu, P. S., and Chen, C. Y. (2010). Butyl benzyl phthalate suppresses the ATPinduced cell proliferation in human osteosarcoma HOS cells. Toxicol. Appl. Pharmacol. 244, 308-314. doi: 10.1016/j.taap.2010.01.007

Liu, R., Ma, S., Lu, Z., Shen, H., Sun, L., and Wei, M. (2015). The ADP antagonist MRS2179 regulates the phenotype of smooth muscle cells to limit intimal hyperplasia. Cardiovasc. Drugs Ther. 29, 23-29. doi: 10.1007/s10557-0146561-6

Liu, R., Wang, H., Xu, B., Chen, W., Turlova, E., Dong, N., et al. (2016). Cerebrovascular safety of sulfonylureas: the role of KATP channels in neuroprotection and the risk of stroke in patients with type 2 diabetes. Diabetes Metab. Res. Rev. 65, 2795-2809. doi: 10.2337/db15-1737

Liu, S., Shi, Q., Zhu, Q., Zou, T., Li, G., Huang, A., et al. (2015). P2X7 receptor of rat dorsal root ganglia is involved in the effect of moxibustion on visceral hyperalgesia. Purinergic Signal. 11, 161-169. doi: 10.1007/s11302-014-9439-y

Liu, S., Zou, L., Xie, J., Xie, W., Wen, S., Xie, Q., et al. (2016). LncRNA NONRATT021972 siRNA regulates neuropathic pain behaviors in type 2 diabetic rats through the $\mathrm{P}_{2} \mathrm{X}_{7}$ receptor in dorsal root ganglia. Mol. Brain 9, 44. doi: 10.1186/s13041-016-0226-2

Liu, W., Ao, Q., Guo, Q., He, W., Peng, L., Jiang, J., et al. (2017). miR-9 mediates CALHM1-activated ATP-P2X7R signal in painful diabetic neuropathy rats. Mol. Neurobiol. 54, 922-929. doi: 10.1007/s12035-016-9700-1

Liu, X., Wang, C., Fujita, T., Malmstrom, H. S., Nedergaard, M., Ren, Y. F., et al. (2015). External dentin stimulation induces ATP release in human teeth. J. Dent. Res. 94, 1259-1266. doi: 10.1177/0022034515592858

Liu, Z., Liu, Y., Xu, L., An, H., Chang, Y., Yang, Y., et al. (2015). P2X7 receptor predicts postoperative cancer-specific survival of patients with clear-cell renal cell carcinoma. Cancer Sci. 106, 1224-1231. doi: 10.1111/cas.12736

Liverani, E. (2017). Lung injury during LPS-induced inflammation occurs independently of the receptor P2Y1. Purinergic Signal. 13, 119-125. doi: 10.1007/s11302-016-9543-2

Liverani, E., Rico, M. C., Tsygankov, A. Y., Kilpatrick, L. E., and Kunapuli, S. P. (2016). P2Y12 receptor modulates sepsis-induced inflammation. Arterioscler. Thromb. Vasc. Biol. 36, 961-971. doi: 10.1161/ATVBAHA.116.307401

Loma, P., Guzman-Aranguez, A., Perez De Lara, M. J., and Pintor, J. (2015). Diadenosine tetraphosphate improves adrenergic anti-glaucomatous drug delivery and efficiency. Exp. Eye Res. 134, 141-147. doi: 10.1016/j.exer.2015. 02.014

Lu, J., Cui, J., Li, X., Wang, X., Zhou, Y., Yang, W., et al. (2016). An antiParkinson's disease drug via targeting adenosine A2A receptor enhances amyloid- $\beta$ generation and $\gamma$-secretase activity. PLOS ONE 11:e0166415. doi: 10.1371/journal.pone. 0166415

Lu, W., Hu, H., Sévigny, J., Gabelt, B. T., Kaufman, P. L., Johnson, E. C., et al. (2015). Rat, mouse, and primate models of chronic glaucoma show sustained elevation of extracellular ATP and altered purinergic signaling in the posterior eye. Invest. Ophthalmol. Vis. Sci. 56, 3075-3083. doi: 10.1167/iovs.14-15891

Luo, F., Le, N. B., Mills, T., Chen, N. Y., Karmouty-Quintana, H., Molina, J. G., et al. (2016). Extracellular adenosine levels are associated with the progression and exacerbation of pulmonary fibrosis. FASEB J. 30, 874-883. doi: 10.1096/fj. $15-274845$

Luo, Y., Feng, C., Wu, J., Wu, Y., Liu, D., Wu, J., et al. (2016). P2Y1, P2Y2, and TRPV1 receptors are increased in diarrhea-predominant irritable bowel syndrome and P2Y2 correlates with abdominal pain. Dig. Dis. Sci. 61, 2878-2886. doi: 10.1007/s10620-016-4211-5
Ma, B., Jiang, Q., Li, W., and Li, Z. (2016). Estrogen receptor beta (ER $\beta$ ) activation plays a therapeutic role in murine models of inflammatory bowel disease (IBD) via inhibiting $\mathrm{P} 2 \mathrm{X} 3$ and $\mathrm{P} 2 \mathrm{X} 7$ receptors. FASEB J. 30, 1023-1023.

Ma, D. F., Kondo, T., Nakazawa, T., Niu, D. F., Mochizuki, K., Kawasaki, T., et al. (2010). Hypoxia-inducible adenosine A2B receptor modulates proliferation of colon carcinoma cells. Hum. Pathol. 41, 1550-1557. doi: 10.1016/j.humpath. 2010.04.008

Ma, M., Ren, Q., Zhang, J. C., and Hashimoto, K. (2014). Effects of brilliant blue G on serum tumor necrosis factor- $\alpha$ levels and depression-like behavior in mice after lipopolysaccharide administration. Clin. Psychopharmacol. Neurosci. 12, 31-36. doi: 10.9758/cpn.2014.12.1.31

Ma, X., Pan, X., Wei, Y., Tan, B., Yang, L., Ren, H., et al. (2016). Chemotherapyinduced uridine diphosphate release promotes breast cancer metastasis through P2Y6 activation. Oncotarget 7, 29036-29050. doi: 10.18632/oncotarget.8664

$\mathrm{Ma}, \mathrm{X} ., \mathrm{Xu}, \mathrm{T} ., \mathrm{Xu}, \mathrm{H}$., and Jiang, W. (2015). Blockade and reversal of spinal morphine tolerance by $\mathrm{P} 2 \mathrm{X} 3$ receptor antagonist. Behav. Pharmacol. 26, 260-267. doi: 10.1097/FBP.0000000000000105

Mac Mullan, P. A., Peace, A. J., Madigan, A. M., Tedesco, A. F., Kenny, D., and Mccarthy, G. M. (2010). Platelet hyper-reactivity in active inflammatory arthritis is unique to the adenosine diphosphate pathway: a novel finding and potential therapeutic target. Rheumatology 49, 240-245. doi: 10.1093/ rheumatology/kep377

Macle, L., Khairy, P., Weerasooriya, R., Novak, P., Verma, A., Willems, S., et al. (2015). Adenosine-guided pulmonary vein isolation for the treatment of paroxysmal atrial fibrillation: an international, multicentre, randomised superiority trial. Lancet 386, 672-679. doi: 10.1016/S0140-6736(15) 60026-5

Magni, G., Merli, D., Verderio, C., Abbracchio, M. P., and Ceruti, S. (2015). $\mathrm{P} 2 \mathrm{Y} 2$ receptor antagonists as anti-allodynic agents in acute and sub-chronic trigeminal sensitization: role of satellite glial cells. Glia 63, 1256-1269. doi: 10.1002/glia.22819

Mahi, N., Kumar, A., Jaggi, A. S., Singh, N., and Dhawan, R. (2015). Possible role of pannexin 1/P2x7 purinoceptor in neuroprotective mechanism of ischemic postconditioning in mice. J. Surg. Res. 196, 190-199. doi: 10.1016/j.jss.2015. 02.050

Maminishkis, A., Jalickee, S., Blaug, S. A., Rymer, J., Yerxa, B. R., Peterson, W. M., et al. (2002). The P2Y2 receptor agonist INS37217 stimulates RPE fluid transport in vitro and retinal reattachment in rat. Invest. Ophthalmol. Vis. Sci. $43,3555-3566$.

Manzoor, S., Akhtar, U., Naseem, S., Khalid, M., Mazhar, M., Parvaiz, F., et al. (2016). Ionotropic purinergic receptors P2X4 and P2X7: proviral or antiviral? An insight into P2X receptor signaling and hepatitis C virus infection. Viral Immunol. 29, 401-408. doi: 10.1089/vim.2016.0008

Marchenkova, A., Van Den Maagdenberg, A. M., and Nistri, A. (2016). Loss of inhibition by brain natriuretic peptide over $\mathrm{P} 2 \mathrm{X} 3$ receptors contributes to enhanced spike firing of trigeminal ganglion neurons in a mouse model of familial hemiplegic migraine type-1. Neuroscience 331, 197-205. doi: 10.1016/j. neuroscience.2016.06.034

Martinez, C. G., Zamith-Miranda, D., Da Silva, M. G., Ribeiro, K. C., Brandão, I. T., Silva, C. L., et al. (2015). P2x7 purinergic signaling in dilated cardiomyopathy induced by auto-immunity against muscarinic M2 receptors: autoantibody levels, heart functionality and cytokine expression. Sci. Rep. 5:16940. doi: 10.1038/srep 16940

Martinez, N. A., Ayala, A. M., Martinez, M., Martinez-Rivera, F. J., Miranda, J. D., and Silva, W. I. (2016). Caveolin-1 regulates the P2Y2 receptor signaling in human $1321 \mathrm{~N} 1$ astrocytoma cells. J. Biol. Chem. 291, 12208-12222. doi: 10.1074/jbc.M116.730226

Martins, J. P., Silva, R. B., Coutinho-Silva, R., Takiya, C. M., Battastini, A. M., Morrone, F. B., et al. (2012). The role of P2X7 purinergic receptors in inflammatory and nociceptive changes accompanying cyclophosphamideinduced haemorrhagic cystitis in mice. Br. J. Pharmacol. 165, 183-196. doi: 10.1111/j.1476-5381.2011.01535.x

Masahiro, K., Nakamachi, Y., Kosaka, H., Nakazawa, T., Tsuji, G., and Kumagai, S. (2003). Modification of cytokine milieu in rheumatoid arthritis by signalling through A2A adenosine receptors. FASEB J. 17, C69.

Masino, S. A., Kawamura, M. Jr., Cote, J. L., Williams, R. B., and Ruskin, D. N. (2013). Adenosine and autism: a spectrum of opportunities. Neuropharmacology 68, 116-121. doi: 10.1016/j.neuropharm.2012.08.013 
Masuch, A., Shieh, C. H., Van Rooijen, N., Van Calker, D., and Biber, K. (2016). Mechanism of microglia neuroprotection: involvement of $\mathrm{P} 2 \mathrm{X} 7, \mathrm{TNF} \alpha$, and valproic acid. Glia 64, 76-89. doi: 10.1002/glia.22904

Matos, M., Shen, H. Y., Augusto, E., Wang, Y., Wei, C. J., Wang, Y. T., et al. (2015). Deletion of adenosine A2A receptors from astrocytes disrupts glutamate homeostasis leading to psychomotor and cognitive impairment: relevance to schizophrenia. Biol. Psychiatry 78, 763-774. doi: 10.1016/j.biopsych.2015. 02.026

Matsumoto, T., Goulopoulou, S., Taguchi, K., Tostes, R. C., and Kobayashi, T. (2015). Constrictor prostanoids and uridine adenosine tetraphosphate: vascular mediators and therapeutic targets in hypertension and diabetes. Br. J. Pharmacol. 172, 3980-4001. doi: 10.1111/bph.13205

Matsumura, Y., Yamashita, T., Sasaki, A., Nakata, E., Kohno, K., Masuda, T., et al. (2016). A novel P2X4 receptor-selective antagonist produces anti-allodynic effect in a mouse model of herpetic pain. Sci. Rep. 6:32461. doi: 10.1038/ srep32461

Matta, C., Fodor, J., Miosge, N., Takács, R., Juhász, T., Rybaltovszki, H., et al. (2015). Purinergic signalling is required for calcium oscillations in migratory chondrogenic progenitor cells. Pflugers Arch. 467, 429-442. doi: 10.1007/ s00424-014-1529-8

Maynard, J. P., Lee, J. S., Sohn, B. H., Yu, X., Lopez-Terrada, D., Finegold, M. J., et al. (2015). P2X3 purinergic receptor overexpression is associated with poor recurrence-free survival in hepatocellular carcinoma patients. Oncotarget 6, 41162-41179. doi: 10.18632/oncotarget.6240

Mazzon, E., Esposito, E., Impellizzeri, D., Di Paola, R., Melani, A., Bramanti, P., et al. (2011). CGS 21680, an agonist of the adenosine (A2A) receptor, reduces progression of murine type II collagen-induced arthritis. J. Rheumatol. 38, 2119-2129. doi: 10.3899/jrheum.110111

McGovern, A. E., and Mazzone, S. B. (2014). Neural regulation of inflammation in the airways and lungs. Auton. Neurosci. 182, 95-101. doi: 10.1016/j.autneu. 2013.12.008

McLarnon, J. G. (2017). Roles of purinergic P2X7 receptor in glioma and microglia in brain tumors. Cancer Lett. 402, 93-99. doi: 10.1016/j.canlet.2017.05.004

McPhee, M. D., and Whiting, S. J. (1989). The effect of adenosine and adenosine analogues on methylxanthine-induced hypercalciuria in the rat. Can. J. Physiol. Pharmacol. 67, 1278-1282. doi: 10.1139/y89-203

Mediavilla-Varela, M., Luddy, K., Noyes, D., Khalil, F. K., Neuger, A. M., Soliman, H., et al. (2013). Antagonism of adenosine A2A receptor expressed by lung adenocarcinoma tumor cells and cancer associated fibroblasts inhibits their growth. Cancer Biol. Ther. 14, 860-868. doi: 10.4161/cbt.25643

Mendieta-Condado, E., Chagoya de Sanchez, V., and Hernandez-Munoz, R. (2007). Adenosine can accelerate the cell cycle during rat liver regeneration induced by partial hepatectomy. J. Hepatol. 46, S142-S143. doi: 10.1124/jpet. 109.156620

Menezes, C. B., and Tasca, T. (2016). Trichomoniasis immunity and the involvement of the purinergic signaling. Biomed. J. 39, 234-243. doi: 10.1016/j. bj.2016.06.007

Meng, E., Lin, W. Y., Lee, W. C., and Chuang, Y. C. (2012). Pathophysiology of overactive bladder. LUTS 4, 48-55. doi: 10.1111/j.1757-5672.2011.00122.x

Menzies, R. I., Tam, F. W., Unwin, R. J., and Bailey, M. A. (2017). Purinergic signaling in kidney disease. Kidney Int. 91, 315-323. doi: 10.1016/j.kint.2016. 08.029

Menzies, R. I., Unwin, R. J., and Bailey, M. A. (2015). Renal P2 receptors and hypertension. Acta Physiol. 213, 232-241. doi: 10.1111/apha.12412

Merighi, S., Borea, P. A., and Gessi, S. (2015). Adenosine receptors and diabetes: focus on the A2B adenosine receptor subtype. Pharmacol. Res. 99, 229-236. doi: 10.1016/j.phrs.2015.06.015

Merighi, S., Borea, P. A., Varani, K., and Gessi, S. (2017). Deregulation of adenosine receptors in psoriatic epidermis: an option for therapeutic treatment. J. Invest. Dermatol. 137, 11-13. doi: 10.1016/j.jid.2016.08.001

Merighi, S., Simioni, C., Gessi, S., Varani, K., Mirandola, P., Tabrizi, M. A., et al. (2009). A2B and A3 adenosine receptors modulate vascular endothelial growth factor and interleukin-8 expression in human melanoma cells treated with etoposide and doxorubicin. Neoplasia 11, 1064-1073. doi: 10.1593/neo.09768

Metzger, M. W., Walser, S. M., Aprile-Garcia, F., Dedic, N., Chen, A., Holsboer, F., et al. (2017). Genetically dissecting P2rx7 expression within the central nervous system using conditional humanized mice. Purinergic Signal. 13, 153-170. doi: $10.1007 / \mathrm{s} 11302-016-9546-\mathrm{z}$
Meyer, C. H., Hotta, K., Peterson, W. M., Toth, C. A., and Jaffe, G. J. (2002). Effect of INS37217, a P2Y2 receptor agonist, on experimental retinal detachment and electroretinogram in adult rabbits. Invest. Ophthalmol. Vis. Sci. 43, 3567-3574.

Michalak, A., and Biala, G. (2016). Alcohol dependence - neurobiology and treatment. Acta Pol. Pharm. 73, 3-12.

Micioni Di Bonaventura, M. V., Cifani, C., Lambertucci, C., Volpini, R., Cristalli, G., Froldi, R., et al. (2012). Effects of A2A adenosine receptor blockade or stimulation on alcohol intake in alcohol-preferring rats. Psychopharmacology 219, 945-957. doi: 10.1007/s00213-011-2430-1

Milicic, I., Buckner, S. A., Daza, A., Coghlan, M., Fey, T. A., Brune, M. E., et al. (2006). Pharmacological characterization of urinary bladder smooth muscle contractility following partial bladder outlet obstruction in pigs. Eur. J. Pharmacol. 532, 107-114. doi: 10.1016/j.ejphar.2005.12.076

Minns, M. S., Teicher, G., Rich, C. B., and Trinkaus-Randall, V. (2016). Purinoreceptor P2X7 regulation of $\mathrm{Ca}^{2+}$ mobilization and cytoskeletal rearrangement is required for corneal reepithelialization after injury. Am. J. Pathol. 186, 285-296. doi: 10.1016/j.ajpath.2015.10.006

Minns, M. S., and Trinkaus-Randall, V. (2016). Purinergic signaling in corneal wound healing: a tale of 2 receptors. J. Ocul. Pharmacol. Ther. 32, 498-503. doi: 10.1089/jop.2016.0009

Miranda, M. F., Hamani, C., De Almeida, A. C., Amorim, B. O., Macedo, C. E., Fernandes, M. J., et al. (2014). Role of adenosine in the antiepileptic effects of deep brain stimulation. Front. Cell. Neurosci. 8:312. doi: 10.3389/fncel.2014. 00312

Miras-Portugal, M. T., Diaz-Hernandez, J. I., Gomez-Villafuertes, R., DiazHernandez, M., Artalejo, A. R., and Gualix, J. (2015). Role of P2X7 and P2Y2 receptors on $\alpha$-secretase-dependent APP processing: control of amyloid plaques formation "in vivo" by P2X7 receptor. Comput. Struct. Biotechnol. J. 13, 176-181. doi: 10.1016/j.csbj.2015.02.005

Mishra, A., Guo, Y., Zhang, L., More, S., Weng, T., Chintagari, N. R., et al. (2016). A critical role for P2X7 receptor-induced VCAM-1 shedding and neutrophil infiltration during acute lung injury. J. Immunol. 197, 2828-2837. doi: 10.4049/ jimmunol.1501041

Mittal, R., Chan, B., Grati, M., Mittal, J., Patel, K., Debs, L. H., et al. (2016). Molecular structure and regulation of P2X receptors with a special emphasis on the role of P2X2 in the auditory system. J. Cell. Physiol. 231, 1656-1670. doi: $10.1002 /$ jcp. 25274

Molck, C., Ryall, J., Failla, L. M., Coates, J. L., Pascussi, J. M., Heath, J. K., et al. (2016). The A2b adenosine receptor antagonist PSB-603 promotes oxidative phosphorylation and ROS production in colorectal cancer cells via adenosine receptor-independent mechanism. Cancer Lett. 383, 135-143. doi: 10.1016/j. canlet.2016.09.018

Molina, C. E., Llach, A., Herraiz-Martínez, A., Tarifa, C., Barriga, M., Wiegerinck, R. F., et al. (2016). Prevention of adenosine A2A receptor activation diminishes beat-to-beat alternation in human atrial myocytes. Basic Res. Cardiol. 111, 5. doi: 10.1007/s00395-015-0525-2

Molz, S., Olescowicz, G., Kraus, J. R., Ludka, F. K., and Tasca, C. I. (2015). Purine receptors are required for DHA-mediated neuroprotection against oxygen and glucose deprivation in hippocampal slices. Purinergic Signal. 11, 117-126. doi: 10.1007/s11302-014-9438-z

Monção-Ribeiro, L. C., Faffe, D. S., Santana, P. T., Vieira, F. S., Da Graca, C. L., Marques-Da-Silva, C., et al. (2014). P2X7 receptor modulates inflammatory and functional pulmonary changes induced by silica. PLOS ONE 9:e110185. doi: 10.1371/journal.pone.0110185

Montesinos, M. C., Desai-Merchant, A., and Cronstein, B. N. (2015). Promotion of wound healing by an agonist of adenosine $\mathrm{A} 2 \mathrm{~A}$ receptor is dependent on tissue plasminogen activator. Inflammation 38, 2036-2041. doi: 10.1007/s10753-0150184-3

Morandini, A. C., Savio, L. E., and Coutinho-Silva, R. (2014). The role of P2X7 receptor in infectious inflammatory diseases and the influence of ectonucleotidases. Biomed. J. 37, 169-177. doi: 10.4103/2319-4170.127803

Morello, S., Sorrentino, R., Montinaro, A., Luciano, A., Maiolino, P., Ngkelo, A., et al. (2011). NK1.1 ${ }^{+}$cells and $\mathrm{CD} 8^{+} \mathrm{T}$ cells mediate the antitumor activity of Cl-IB-MECA in a mouse melanoma model. Neoplasia 13, 365-373. doi: $10.1593 /$ neo. 101628

Mori, A. (2014). Mode of action of adenosine A2A receptor antagonists as symptomatic treatment for Parkinson's disease. Int. Rev. Neurobiol. 119, 87-116. doi: 10.1016/B978-0-12-801022-8.00004-0 
Morrone, F. B., Gehring, M. P., and Nicoletti, N. F. (2016). Calcium channels and associated receptors in malignant brain tumor therapy. Mol. Pharmacol. 90, 403-409. doi: 10.1124/mol.116.103770

Mrazek, F., Gallo, J., Stahelova, A., and Petrek, M. (2010). Functional variants of the P2RX7 gene, aseptic osteolysis, and revision of the total hip arthroplasty: a preliminary study. Hum. Immunol. 71, 201-205. doi: 10.1016/j.humimm.2009. 10.013

Mujoomdar, M., Bennett, A., Hoskin, D., and Blay, J. (2004). Adenosine stimulation of proliferation of breast carcinoma cell lines: evaluation of the $[3 \mathrm{H}]$ thymidine assay system and modulatory effects of the cellular microenvironment in vitro. J. Cell. Physiol. 201, 429-438. doi: 10.1002/jcp. 20089

Mulryan, K., Gitterman, D. P., Lewis, C. J., Vial, C., Leckie, B. J., Cobb, A. L., et al. (2000). Reduced vas deferens contraction and male infertility in mice lacking P2X1 receptors. Nature 403, 86-89. doi: 10.1038/47495

Mumtaz, F. H., Lau, D. H., Siddiqui, E. J., Morgan, R. J., Thompson, C. S., and Mikhailidis, D. P. (2006). Changes in cholinergic and purinergic neurotransmission in the diabetic rabbit bladder. In Vivo 20, 1-4.

Munoz, A., Romain, Z., Munch, E., Gangitano, D., Boone, T., Smith, C., et al. (2008). Changes in purinergic and nitrergic sensory signals in female rats during early diabetes. Neurourol. Urodyn. 28, 110-111.

Munoz, A., Somogyi, G. T., Boone, T. B., Ford, A. P., and Smith, C. P. (2012), Modulation of bladder afferent signals in normal and spinal cord-injured rats by purinergic P2X3 and P2X2/3 receptors. BJU Int. 110, E409-E414. doi: 10.1111/j.1464-410X.2012.11189.x

Munoz, A., Yazdi, I. K., Tang, X., Rivera, C., Taghipour, N., Grossman, R. G., et al. (2017). Localized inhibition of P2X7R at the spinal cord injury site improves neurogenic bladder dysfunction by decreasing urothelial P2X3R expression in rats. Life Sci. 171, 60-67. doi: 10.1016/j.lfs.2016.12.017

Murakami, S., Yoshida, M., Masunaga, K., Maeda, Y., and Ueda, S. (2008). Change in acetylcholine release from rat bladder with partial outlet obstruction. BJU Int. 101, 633-639. doi: 10.1111/j.1464-410X.2007. 07325.x

Muti, M., Del Grande, C., Musetti, L., Marazziti, D., Turri, M., Cirronis, M., et al. (2015). Serum uric acid levels and different phases of illness in bipolar I patients treated with lithium. Psychiatry Res. 225, 604-608. doi: 10.1016/j.psychres.2014. 11.038

Nagahama, M., Seike, S., Shirai, H., Takagishi, T., Kobayashi, K., Takehara, M., et al. (2015). Role of P2X7 receptor in Clostridium perfringens beta-toxin-mediated cellular injury. Biochim. Biophys. Acta 1850, 2159-2167. doi: 10.1016/j.bbagen. 2015.08.011

Nakagawa, Y., Yoshioka, M., Abe, Y., Uchinami, H., Ohba, T., Ono, K., et al. (2012). Enhancement of liver regeneration by adenosine triphosphate-sensitive $\mathrm{K}+$ channel opener (diazoxide) after partial hepatectomy. Transplantation 93, 1094-1100. doi: 10.1097/TP.0b013e31824ef1d1

Nakamachi, Y., Koshiba, M., Nakazawa, T., Hatachi, S., Saura, R., Kurosaka, M., et al. (2003). Specific increase in enzymatic activity of adenosine deaminase 1 in rheumatoid synovial fibroblasts. Arthritis Rheum. 48, 668-674. doi: 10.1002/ art.10956

Nam, H. W., Bruner, R. C., and Choi, D. S. (2013). Adenosine signaling in striatal circuits and alcohol use disorders. Mol. Cells 36, 195-202. doi: 10.1007/s10059013-0192-9

Nascimento, F., Pousinha, P. A., Correia, A. M., Gomes, R., Sebastião, A. M., and Ribeiro, J. A. (2014). Adenosine A2A receptors activation facilitates neuromuscular transmission in the pre-symptomatic phase of the SOD1(G93A) ALS mice, but not in the symptomatic phase. PLOS ONE 9:e104081. doi: 10.1371/journal.pone. 0104081

Nascimento, F., Sebastião, A. M., and Ribeiro, J. A. (2015). Presymptomatic and symptomatic ALS SOD1(G93A) mice differ in adenosine $\mathrm{A} 1$ and A2A receptormediated tonic modulation of neuromuscular transmission. Purinergic Signal. 11, 471-480. doi: 10.1007/s11302-015-9465-4

Navarro, G., Borroto-Escuela, D. O., Fuxe, K., and Franco, R. (2016). Purinergic signaling in Parkinson's disease. Relevance for treatment. Neuropharmacology 104, 161-168. doi: 10.1016/j.neuropharm.2015.07.024

Naviaux, J. C., Schuchbauer, M. A., Li, K., Wang, L., Risbrough, V. B., Powell, S. B., et al. (2014). Reversal of autism-like behaviors and metabolism in adult mice with single-dose antipurinergic therapy. Transl. Psychiatry 4, e400. doi: $10.1038 /$ tp.2014.33
Naviaux, J. C., Wang, L., Li, K., Bright, A. T., Alaynick, W. A., Williams, K. R., et al. (2015). Antipurinergic therapy corrects the autism-like features in the Fragile $\mathrm{X}$ (Fmr1 knockout) mouse model. Mol. Autism 6:1. doi: 10.1186/2040-2392-6-1

Nelson, S., Kiyono, H., and Kurashima, Y. (2017). Epithelial extracellular ATP: an initiator of immunity to parasitic infections. Immunol. Cell Biol. 95, 117-118. doi: 10.1038/icb.2016.106

Neuhuber, W. L., and Tiegs, G. (2004). Innervation of immune cells: evidence for neuroimmunomodulation in the liver. Anat. Rec. A Discov. Mol. Cell. Evol. Biol. 280, 884-892. doi: 10.1002/ar.a.20093

Neves, A. R., Castelo-Branco, M. T., Figliuolo, V. R., Bernardazzi, C., Buongusto, F., Yoshimoto, A., et al. (2014). Overexpression of ATP-activated P2X7 receptors in the intestinal mucosa is implicated in the pathogenesis of Crohn's disease. Inflamm. Bowel Dis. 20, 444-457. doi: 10.1097/01.MIB.0000441201.10454.06

Ng, S. K., Higashimori, H., Tolman, M., and Yang, Y. (2015). Suppression of adenosine 2a receptor (A2aR)-mediated adenosine signaling improves disease phenotypes in a mouse model of amyotrophic lateral sclerosis. Exp. Neurol. 267, 115-122. doi: 10.1016/j.expneurol.2015.03.004

Ni, R., Zheng, D., Xiong, S., Hill, D. J., Sun, T., Gardiner, R. B., et al. (2016). Mitochondrial calpain-1 disrupts ATP synthase and induces superoxide generation in type 1 diabetic hearts: a novel mechanism contributing to diabetic cardiomyopathy. Diabetes Metab. Res. Rev. 65, 255-268. doi: 10.2337/db150963

Ning, C., Wen, J., Zhang, Y., Blackburn, M., Kellems, R., and Xia, Y. (2012a). Impaired adenosine signaling contributes to erectile dysfunction. J. Sex. Med. 9, 183-298. doi: 10.1161/ATVBAHA.111.226803

Ning, C., Zhang, Y., Wen, J., Blackburn, M., Kellems, R., and Xia, Y. (2012b). Adenosine signaling in priapism and novel therapies. J. Sex. Med. 9, 223.

Noronha-Matos, J. B., Morais, T., Trigo, D., Timóteo, M. A., Magalhães-Cardoso, M. T., Oliveira, L., et al. (2011). Tetanic failure due to decreased endogenous adenosine A2A tonus operating neuronal Cav 1 (L-type) influx in Myasthenia gravis. J. Neurochem. 117, 797-811. doi: 10.1111/j.1471-4159.2011.07216.x

Novitskaya, T., Chepurko, E., Covarrubias, R., Novitskiy, S., Ryzhov, S. V., Feoktistov, I., et al. (2016). Extracellular nucleotide regulation and signaling in cardiac fibrosis. J. Mol. Cell. Cardiol. 93, 47-56. doi: $10.1016 /$ j.yjmcc.2016.02.010

Nylander, S., and Schulz, R. (2016). Effects of P2Y12 receptor antagonists beyond platelet inhibition - comparison of ticagrelor with thienopyridines. $\mathrm{Br}$. J. Pharmacol. 173, 1163-1178. doi: 10.1111/bph.13429

Ochoa-Cortes, F., Liñán-Rico, A., Jacobson, K. A., and Christofi, F. L. (2014). Potential for developing purinergic drugs for gastrointestinal diseases. Inflamm. Bowel Dis. 20, 1259-1287. doi: 10.1097/MIB.0000000000000047

Ohshima, Y., Tsukimoto, M., Takenouchi, T., Harada, H., Suzuki, A., Sato, M., et al. (2010). $\gamma$-Irradiation induces P2X7 receptor-dependent ATP release from B16 melanoma cells. Biochim. Biophys. Acta 1800, 40-46. doi: 10.1016/j.bbagen. 2009.10.008

Olatunji, O. J., Feng, Y., Olatunji, O. O., Tang, J., Ouyang, Z., Su, Z., et al. (2016). Neuroprotective effects of adenosine isolated from Cordyceps cicadae against oxidative and ER stress damages induced by glutamate in PC12 cells. Environ. Toxicol. Pharmacol. 44, 53-61. doi: 10.1016/j.etap.2016. 02.009

Oliveira, A. G., Marques, P. E., Amaral, S. S., Quintão, J. L., Cogliati, B., Dagli, M. L., et al. (2013). Purinergic signalling during sterile liver injury. Liver Int. 33, 353-361. doi: 10.1111/liv.12109

Oliveira, L., Correia, A., Costa, A. C., Guerra-Gomes, S., Ferreirinha, F., Magalhães-Cardoso, M. T., et al. (2015). Deficits in endogenous adenosine formation by ecto- $5^{\prime}$-nucleotidase/CD73 impair neuromuscular transmission and immune competence in experimental autoimmune myasthenia gravis. Mediators Inflamm. 2015:460610. doi: 10.1155/2015/460610

Olivier, E., Dutot, M., Regazzetti, A., Leguillier, T., Dargere, D., Auzeil, N., et al. (2016). P2X7-pannexin-1 and amyloid $\beta$-induced oxysterol input in human retinal cell: role in age-related macular degeneration? Biochimie 127, 70-78. doi: 10.1016/j.biochi.2016.04.014

Ondrakova, M., Knot, J., Ulman, J., Maly, M., and Motovska, Z. (2016). Efficacy of P2Y12 receptor antagonists in patients with atrial fibrillation according to the CHA2DS2VASc score. Int. J. Cardiol. 207, 84-86. doi: 10.1016/j.ijcard.2016. 01.039

Ongaro, A., Varani, K., Masieri, F. F., Pellati, A., Massari, L., Cadossi, R. et al. (2012). Electromagnetic fields (EMFs) and adenosine receptors modulate 
prostaglandin $\mathrm{E}(2)$ and cytokine release in human osteoarthritic synovial fibroblasts. J. Cell. Physiol. 227, 2461-2469. doi: 10.1002/jcp.22981

O’Reilly, B. A., Kosaka, A. H., Knight, G. E., Chang, T. K., Ford, A. P. D. W., Rymer, J. M., et al. (2002). P2X receptors and their role in female idiopathic detrusor instability. J. Urol. 167, 157-164. doi: 10.1016/S0022-5347(05)65403-1

Orriss, I. R., Arnett, T. R., and Russell, R. G. (2016). Pyrophosphate: a key inhibitor of mineralisation. Curr. Opin. Pharmacol. 28, 57-68. doi: 10.1016/j.coph.2016. 03.003

Orth, S. R., Amann, K., Strojek, K., and Ritz, E. (2001). Sympathetic overactivity and arterial hypertension in renal failure. Nephrol. Dial. Transplant. 16(Suppl. 1), 67-69. doi: 10.1093/ndt/16.suppl_1.67

Ortiz, R., Ulrich, H., Zarate, C. A. Jr., and Machado-Vieira, R. (2015). Purinergic system dysfunction in mood disorders: a key target for developing improved therapeutics. Prog. Neuropsychopharmacol. Biol. Psychiatry 57, 117-131. doi: 10.1016/j.pnpbp.2014.10.016

Ou, Z., Sun, Y., Lin, L., You, N., Liu, X., Li, H., et al. (2016). Olig2targeted G-protein-coupled receptor Gpr17 regulates oligodendrocyte survival in response to lysolecithin-induced demyelination. J. Neurosci. 36, 10560-10573. doi: 10.1523/JNEUROSCI.0898-16.2016

Oury, C., Lecut, C., Hego, A., Wéra, O., and Delierneux, C. (2015). Purinergic control of inflammation and thrombosis: role of P2X1 receptors. Comput. Struct. Biotechnol. J. 13, 106-110. doi: 10.1016/j.csbj.2014.11.008

Oyarzun, C., Garrido, W., Alarcon, S., Yanez, A., Sobrevia, L., Quezada, C., et al. (2017). Adenosine contribution to normal renal physiology and chronic kidney disease. Mol. Aspects Med. 55, 75-89. doi: 10.1016/j.mam.2017.01.004

Ozaki, T., Muramatsu, R., Sasai, M., Yamamoto, M., Kubota, Y., Fujinaka, T., et al. (2016). The P2X4 receptor is required for neuroprotection via ischemic preconditioning. Sci. Rep. 6:25893. doi: 10.1038/srep25893

Pacheco, P. A., Faria, R. X., Ferreira, L. G., and Paixão, I. C. (2014). Putative roles of purinergic signaling in human immunodeficiency virus-1 infection. Biol. Direct 9:21. doi: $10.1186 / 1745-6150-9-21$

Pandolfi, J. B., Ferraro, A. A., Sananez, I., Gancedo, M. C., Baz, P., Billordo, L. A., et al. (2016). ATP-induced inflammation drives tissue-resident Th17 cells in metabolically unhealthy obesity. J. Immunol. 196, 3287-3296. doi: 10.4049/ jimmunol.1502506

Panjehpour, M., Hemati, S., and Forghani, M. A. (2012). Expression of A1 and A3 adenosine receptors in human breast tumors. Tumori 98, 137-141. doi: 10.1700/1053.11512

Parabucki, A., Savic, D., Laketa, D., Pekovic, S., Stojiljkovic, M., Nedeljkovic, N., et al. (2014). Expression of major ectonucleotidases after cortical stab brain injury in rats: a real-time PCR study. Arch. Biol. Sci. Belgrade 66, 149-155. doi: 10.2298/ABS1401148P

Patel, A., and Honoré, E. (2010). Polycystins and renovascular mechanosensory transduction. Nat. Rev. Nephrol. 6, 530-538. doi: 10.1038/nrneph.2010.97

Pedata, F., Dettori, I., Coppi, E., Melani, A., Fusco, I., Corradetti, R., et al. (2016). Purinergic signalling in brain ischemia. Neuropharmacology 104, 105-130. doi: 10.1016/j.neuropharm.2015.11.007

Pedata, F., Pugliese, A. M., Coppi, E., Dettori, I., Maraula, G., Cellai, L., et al. (2014). Adenosine A2A receptors modulate acute injury and neuroinflammation in brain ischemia. Mediators Inflamm. 2014:805198. doi: 10.1155/2014/805198

Pei, H., and Linden, J. (2016). Adenosine influences myeloid cells to inhibit aeroallergen sensitization. Am. J. Physiol. Lung Cell. Mol. Physiol. 310, L985-L992. doi: 10.1152/ajplung.00330.2015

Pelleg, A., Schulman, E. S., and Barnes, P. J. (2016). Extracellular adenosine 5'triphosphate in obstructive airway diseases. Chest 150, 908-915. doi: 10.1016/j. chest.2016.06.045

Peng, H., Zou, L., Xie, J., Wu, H., Wu, B., Zhu, G., et al. (2017). IncRNA NONRATT021972 siRNA decreases diabetic neuropathic pain mediated by the P2X3 receptor in dorsal root ganglia. Mol. Neurobiol. 54, 511-523. doi: $10.1007 / \mathrm{s} 12035-015-9632-1$

Peng, W., Cotrina, M. L., Han, X., Yu, H., Bekar, L., Blum, L., et al. (2009). Systemic administration of an antagonist of the ATP-sensitive receptor P2X7 improves recovery after spinal cord injury. Proc. Natl. Acad. Sci. U.S.A. 106, 12489-12493. doi: 10.1073/pnas.0902531106

Pereira, V. S., Casarotto, P. C., Hiroaki-Sato, V. A., Sartim, A. G., Guimarães, F. S., and Joca, S. R. (2013). Antidepressant- and anticompulsive-like effects of purinergic receptor blockade: involvement of nitric oxide. Eur. Neuropsychopharmacol. 23, 1769-1778. doi: 10.1016/j.euroneuro.2013.01.008
Pérez de Lara, M. J., Guzmán-Aránguez, A., De La Villa, P., Díaz-Hernández, J. I., Miras-Portugal, M. T., and Pintor, J. (2015). Increased levels of extracellular ATP in glaucomatous retinas: possible role of the vesicular nucleotide transporter during the development of the pathology. Mol. Vis. 21, 1060-1070.

Perez-Aso, M., Mediero, A., Low, Y. C., Levine, J., and Cronstein, B. N. (2016). Adenosine A2A receptor plays an important role in radiation-induced dermal injury. FASEB J. 30, 457-465. doi: 10.1096/fj.15-280388

Pérez-Sen, R., Queipo, M. J., Morente, V., Ortega, F., Delicado, E. G., and Miras-Portugal, M. T. (2015). Neuroprotection mediated by P2Y13 nucleotide receptors in neurons. Comput. Struct. Biotechnol. J. 13, 160-168. doi: 10.1016/j. csbj.2015.02.002

Persson, P., Friederich-Persson, M., Fasching, A., Hansell, P., Inagi, R., and Palm, F. (2015). Adenosine A2 a receptor stimulation prevents proteinuria in diabetic rats by promoting an anti-inflammatory phenotype without affecting oxidative stress. Acta Physiol. 214, 311-318. doi: 10.1111/apha.12511

Petrasek, J., Iracheta-Vellve, A., Saha, B., Satishchandran, A., Kodys, K., Fitzgerald, K. A., et al. (2015). Metabolic danger signals, uric acid and ATP, mediate inflammatory cross-talk between hepatocytes and immune cells in alcoholic liver disease. J. Leukoc. Biol. 98, 249-256. doi: 10.1189/jlb.3AB1214-590R

Pettengill, M. A., Abdul-Sater, A., Coutinho-Silva, R., and Ojcius, D. M. (2016). Danger signals, inflammasomes, and the intricate intracellular lives of chlamydiae. Biomed. J. 39, 306-315. doi: 10.1016/j.bj.2016.07.001

Phillips, E., Carpenter, C., and Oates, R. D. (2014). Ejaculatory dysfunction. Urol. Clin. North Am. 41, 115-128. doi: 10.1016/j.ucl.2013.08.018

Pijacka, W., Moraes, D. J. A., Ratcliffe, L. E. K., Nightingale, A. K., Hart, E. C., Da Silva, M. P., et al. (2016). Purinergic receptors in the carotid body as a new drug target for controlling hypertension. Nat. Med. 22, 1151-1159. doi: $10.1038 / \mathrm{nm} .4173$

Pinna, A. (2014). Adenosine A2A receptor antagonists in Parkinson's disease: progress in clinical trials from the newly approved istradefylline to drugs in early development and those already discontinued. CNS Drugs 28, 455-474. doi: 10.1007/s40263-014-0161-7

Pintsuk, J., Borroto-Escuela, D. O., Pomierny, B., Wydra, K., Zaniewska, M., Filip, M., et al. (2016). Cocaine self-administration differentially affects allosteric A2A-D2 receptor-receptor interactions in the striatum. Relevance for cocaine use disorder. Pharmacol. Biochem. Behav. 144, 85-91. doi: 10.1016/j. pbb.2016.03.004

Plemel, J. R., Keough, M. B., Duncan, G. J., Sparling, J. S., Yong, V. W., Stys, P. K., et al. (2014). Remyelination after spinal cord injury: is it a target for repair? Prog. Neurobiol. 117, 54-72. doi: 10.1016/j.pneurobio.2014.02.006

Pochmann, D., Rucker, B., Battastini, A. M., and Sarkis, J. J. (2004). Ovariectomy and estradiol replacement therapy alters the adenine nucleotide hydrolysis in rat blood serum. Thromb. Res. 114, 275-281. doi: 10.1016/j.thromres.2004.06.021

Portales-Cervantes, L., Niño-Moreno, P., Doníz-Padilla, L., Baranda-Candido, L., García-Hernández, M., Salgado-Bustamante, M., et al. (2010). Expression and function of the P2X7 purinergic receptor in patients with systemic lupus erythematosus and rheumatoid arthritis. Hum. Immunol. 71, 818-825. doi: 10.1016/j.humimm.2010.05.008

Portales-Cervantes, L., Niño-Moreno, P., Salgado-Bustamante, M., GarcíaHernández, M. H., Baranda-Candido, L., Reynaga-Hernández, E., et al. (2012). The His155Tyr $(489 \mathrm{C}>\mathrm{T})$ single nucleotide polymorphism of P2RX7 gene confers an enhanced function of P2X7 receptor in immune cells from patients with rheumatoid arthritis. Cell. Immunol. 276, 168-175. doi: 10.1016/j.cellimm. 2012.05.005

Pritchard, M., Mandal, P., Chiang, D. J., Ndum, O., and Nagy, L. E. (2011). Adenosine and adenosine signaling contribute to the anti-inflammatory effect of globular adiponectin in macrophages. Hepatology 54, 1099A-1100A.

Puchalowicz, K., Tarnowski, M., Baranowska-Bosiacka, I., Chlubek, D., and Dziedziejko, V. (2014). P2X and P2Y receptors-role in the pathophysiology of the nervous system. Int. J. Mol. Sci. 15, 23672-23704. doi: 10.3390/ ijms 151223672

Qu, W., Zhu, Z. J., Sun, L. Y., Wei, L., Liu, Y., and Zeng, Z. G. (2017). Correlation between survival interval and CD4+ T-cell intracellular ATP levels in liver transplant recipients. Transplant. Proc. 49, 316-321. doi: 10.1016/j. transproceed.2016.11.044

Quezada, C., Alarcón, S., Jaramillo, C., Muñoz, D., Oyarzún, C., and San Martín, R. (2013). Targeting adenosine signaling to treatment of diabetic nephropathy. Curr. Drug Targets 14, 490-496. doi: 10.2174/1389450111314040010 
Rai, B., Kaur, J., Jacobs, R., and Anand, S. C. (2011). Adenosine deaminase in saliva as a diagnostic marker of squamous cell carcinoma of tongue. Clin. Oral Investig. 15, 347-349. doi: 10.1007/s00784-010-0404-z

Ralevic, V. (2015). P2X receptors in the cardiovascular system and their potential as therapeutic targets in disease. Curr. Med. Chem. 22, 851-865. doi: 10.2174/ 0929867321666141215094050

Ralevic, V., and Burnstock, G. (1998). Receptors for purines and pyrimidines. Pharmacol. Rev. 50, 413-492.

Rama Rao, K. V., and Kielian, T. (2015). Neuron-astrocyte interactions in neurodegenerative diseases: role of neuroinflammation. Clin. Exp. Neuroimmunol. 6, 245-263. doi: 10.1111/cen3.12237

Ramos, D. B., Muller, G. C., Rocha, G. B., Dellavia, G. H., Almeida, R. F., Pettenuzzo, L. F., et al. (2016). Intranasal guanosine administration presents a wide therapeutic time window to reduce brain damage induced by permanent ischemia in rats. Purinergic Signal. 12, 149-159. doi: 10.1007/s11302-0159489-9

Ran, H., Duan, W., Gong, Z., Xu, S., Zhu, H., Hou, X., et al. (2015). Critical contribution of adenosine A2A receptors in bone marrow-derived cells to white matter lesions induced by chronic cerebral hypoperfusion. J. Neuropathol. Exp. Neurol. 74, 305-318. doi: 10.1097/NEN.000000000000 0174

Rao, S., Liu, S., Zou, L., Jia, T., Zhao, S., Wu, B., et al. (2017). The effect of sinomenine in diabetic neuropathic pain mediated by the P2X3 receptor in dorsal root ganglia. Purinergic Signal. 13, 227-235. doi: 10.1007/s11302-0169554-z

Rassendren, F., and Audinat, E. (2016). Purinergic signaling in epilepsy. J. Neurosci. Res. 94, 781-793. doi: 10.1002/jnr.23770

Rau, A. R., Ariwodola, O. J., and Weiner, J. L. (2014). Presynaptic adenosine A1 receptors modulate excitatory transmission in the rat basolateral amygdala. Neuropharmacology 77, 465-474. doi: 10.1016/j.neuropharm.2013. 10.029

Rayalam, S., Yang, J. Y., La-Fera, M. A., and Baile, C. A. (2011). Novel molecular targets for prevention of obesity and osteoporosis. J. Nutr. Biochem. 22, 1099-1104. doi: 10.1016/j.jnutbio.2010.11.009

Rech, J. C., Bhattacharya, A., Letavic, M. A., and Savall, B. M. (2016). The evolution of P2X7 antagonists with a focus on CNS indications. Bioorg. Med. Chem. Lett. 26, 3838-3845. doi: 10.1016/j.bmcl.2016.06.048

Reichenbach, A., and Bringmann, A. (2016). Purinergic signaling in retinal degeneration and regeneration. Neuropharmacology 104, 194-211. doi: 10.1016/j.neuropharm.2015.05.005

Reigada, D., Navarro-Ruiz, R. M., Caballero-López, M. J., Del Águila, Á, MuñozGaldeano, T., Maza, R. M., et al. (2017). Diadenosine tetraphosphate (Ap4A) inhibits ATP-induced excitotoxicity: a neuroprotective strategy for traumatic spinal cord injury treatment. Purinergic Signal. 13, 75-87. doi: 10.1007/s11302016-9541-4

Reiss, A. B., and Cronstein, B. N. (2012). Regulation of foam cells by adenosine. Arterioscler. Thromb. Vasc. Biol. 32, 879-886. doi: 10.1161/ATVBAHA.111. 226878

Ren, C., Qiu, C. Y., Gan, X., Liu, T. T., Qu, Z. W., Rao, Z., et al. (2015). Prokineticin 2 facilitates mechanical allodynia induced by $\alpha, \beta$-methylene ATP in rats. Eur. J. Pharmacol. 767, 24-29. doi: 10.1016/j.ejphar.2015.09.047

Ren, M., Liu, Y., Zhao, H., Dong, S., Jiang, Z., Li, K., et al. (2016). Adenosine triphosphate postconditioning is associated with better preserved global and regional cardiac function during myocardial ischemia and reperfusion: a speckle tracking imaging-based echocardiologic study. Cardiovasc. Ther. 34, 343-351. doi: 10.1111/1755-5922.12205

Rial, D., Lara, D. R., and Cunha, R. A. (2014). The adenosine neuromodulation system in schizophrenia. Int. Rev. Neurobiol. 119, 395-449. doi: 10.1016/B9780-12-801022-8.00016-7

Ribeiro, F. F., Xapelli, S., Miranda-Lourenco, C., Tanqueiro, S. R., FonsecaGomes, J., Diogenes, M. J., et al. (2016). Purine nucleosides in neuroregeneration and neuroprotection. Neuropharmacology 104, 226-242. doi: 10.1016/j.neuropharm.2015.11.006

Richards, J. P., Bowles, E. A., Gordon, W. R., Ellsworth, M. L., Stephenson, A. H., and Sprague, R. S. (2015). Mechanisms of C-peptide-mediated rescue of low O2-induced ATP release from erythrocytes of humans with Type 2 diabetes. Am. J. Physiol. Regul. Integr. Comp. Physiol. 308, R411-R418. doi: 10.1152/ ajpregu.00420.2014
Riding, A., and Pullar, C. E. (2016). ATP release and P2Y receptor signaling are essential for keratinocyte galvanotaxis. J. Cell. Physiol. 231, 181-191. doi: $10.1002 /$ jcp. 25070

Ring, S., Enk, A., and Mahnke, K. (2011). A role for adenosine triphosphate in regulating immune responses during melanoma growth. J. Invest. Dermatol. 131, S91.

Roberts, V., Lu, B., Rajakumar, S., Cowan, P. J., and Dwyer, K. M. (2013). The CD39-adenosinergic axis in the pathogenesis of renal ischemia-reperfusion injury. Purinergic Signal. 9, 135-143. doi: 10.1007/s11302-012-9342-3

Roberts, V. H., Webster, R. P., Brockman, D. E., Pitzer, B. A., and Myatt, L. (2007). Post-translational modifications of the P2X4 purinergic receptor subtype in the human placenta are altered in preeclampsia. Placenta 28, 270-277. doi: 10.1016/j.placenta.2006.04.008

Rockenbach, L., Bavaresco, L., Fernandes, F. P., Cappellari, A. R., Barrios, C. H., Bueno, M. F., et al. (2013). Alterations in the extracellular catabolism of nucleotides are involved in the antiproliferative effect of quercetin in human bladder cancer T24 cells. Urol. Oncol. 31, 1204-1211. doi: 10.1016/j.urolonc. 2011.10.009

Rodrigues, A. M., Bergamaschi, C. T., Fernandes, M. J., Paredes-Gamero, E. J., Buri, M. V., Ferreira, A. T., et al. (2014). P2X7 receptor in the kidneys of diabetic rats submitted to aerobic training or to $\mathrm{N}$-acetylcysteine supplementation [corrected]. PLOS ONE 9:e97452. doi: 10.1371/journal.pone.0097452

Rodrigues, L., Miranda, I. M., Andrade, G. M., Mota, M., Cortes, L., Rodrigues, A. G., et al. (2016). Blunted dynamics of adenosine A2A receptors is associated with increased susceptibility to Candida albicans infection in the elderly. Oncotarget 7, 62862-62872. doi: 10.18632/oncotarget.11760

Rodrigues, R. J., Tomé, A. R., and Cunha, R. A. (2015). ATP as a multi-target danger signal in the brain. Front. Neurosci. 9:148. doi: 10.3389/fnins.2015.00148

Rodriguez-Alvarez, N., Jimenez-Mateos, E. M., Engel, T., Quinlan, S., Reschke, C. R., Conroy, R. M., et al. (2017). Effects of P2X7 receptor antagonists on hypoxia-induced neonatal seizures in mice. Neuropharmacology 116, 351-363. doi: 10.1016/j.neuropharm.2017.01.005

Rollini, F., Franchi, F., and Angiolillo, D. J. (2016). Switching P2Y12-receptor inhibitors in patients with coronary artery disease. Nat. Rev. Cardiol. 13, 11-27. doi: 10.1038/nrcardio.2015.113

Rollini, F., Franchi, F., and Angiolillo, D. J. (2017). Drug-drug interactions when switching between intravenous and oral P2Y12 receptor inhibitors: how real is it? JACC Cardiovasc. Interv. 10, 130-132. doi: 10.1016/j.jcin.2016.11.021

Romagnoli, R., Baraldi, P. G., Cruz-Lopez, O., Lopez-Cara, C., Preti, D., Borea, P. A., et al. (2008). The P2X7 receptor as a therapeutic target. Expert Opin. Ther. Targets 12, 647-661. doi: 10.1517/14728222.12.5.647

Rong, W., and Burnstock, G. (2004). Activation of ureter nociceptors by exogenous and endogenous ATP in guinea pig. Neuropharmacology 47, 1093-1101. doi: 10.1016/j.neuropharm.2004.08.003

Rossi, C., Santini, E., Chiarugi, M., Salvati, A., Comassi, M., Vitolo, E., et al. (2014). The complex P2X7 receptor/inflammasome in perivascular fat tissue of heavy smokers. Eur. J. Clin. Invest. 44, 295-302. doi: 10.1111/eci.12232

Rotte, A., Garmann, D., Buss, I., and Jaehde, U. (2010). Effect of extracellular ATP on cisplatin-induced cytotoxicity in human ovarian carcinoma cells. Chemotherapy 56, 1-8. doi: 10.1159/000287351

Roy Chowdhury, U., Bahler, C. K., Holman, B. H., Dosa, P. I., and Fautsch, M. P. (2015). Ocular hypotensive effects of the ATP-sensitive potassium channel opener cromakalim in human and murine experimental model systems. PLOS ONE 10:e0141783. doi: 10.1371/journal.pone.0141783

Roy Chowdhury, U., Viker, K. B., Stoltz, K. L., Holman, B. H., Fautsch, M. P., and Dosa, P. I. (2016). Analogs of the ATP-sensitive potassium (KATP) channel opener cromakalim with in vivo ocular hypotensive activity. J. Med. Chem. 59, 6221-6231. doi: 10.1021/acs.jmedchem.6b00406

Ru, Q., Tian, X., Wu, Y. X., Wu, R. H., Pi, M. S., and Li, C. Y. (2014). Voltagegated and ATP-sensitive $\mathrm{K}+$ channels are associated with cell proliferation and tumorigenesis of human glioma. Oncol. Rep. 31, 842-848. doi: 10.3892/or.2013. 2875

Rubaiy, H. N. (2016). The therapeutic agents that target ATP-sensitive potassium channels. Acta Pharm. 66, 23-34. doi: 10.1515/acph-2016-0006

Ruzsnavszky, O., Telek, A., Gönczi, M., Balogh, A., Remenyik, E., and Csernoch, L. (2011). UV-B induced alteration in purinergic receptors and signaling on HaCaT keratinocytes. J. Photochem. Photobiol. B 105, 113-118. doi: 10.1016/ j.jphotobiol.2011.07.009 
Ryten, M., Yang, S. Y., Dunn, P. M., Goldspink, G., and Burnstock, G. (2004). Purinoceptor expression in regenerating skeletal muscle in the $\mathrm{mdx}$ mouse model of muscular dystrophy and in satellite cell cultures. FASEB J. 18, 1404-1406. doi: 10.1096/fj.03-1175fje

Sacco, E., Recupero, S. M., Bientinesi, R., Palermo, G., D’agostino, D., Currò, D., et al. (2015). Pioneering drugs for overactive bladder and detrusor overactivity: ongoing research and future directions. World J. Obstet. Gynecol. 4, 24-39.

Sachdeva, S., and Gupta, M. (2013). Adenosine and its receptors as therapeutic targets: an overview. Saudi. Pharm. J. 21, 245-253. doi: 10.1016/j.jsps.2012. 05.011

Sadovnick, A. D., Gu, B. J., Traboulsee, A. L., Bernales, C. Q., Encarnacion, M., Yee, I. M., et al. (2017). Purinergic receptors P2RX4 and P2RX7 in familial multiple sclerosis. Hum. Mutat. 38, 736-744. doi: 10.1002/humu.23218

Sáez-Orellana, F., Godoy, P. A., Bastidas, C. Y., Silva-Grecchi, T., Guzmán, L., Aguayo, L. G., et al. (2016). ATP leakage induces P2XR activation and contributes to acute synaptic excitotoxicity induced by soluble oligomers of $\beta$-amyloid peptide in hippocampal neurons. Neuropharmacology 100, 116-123. doi: 10.1016/j.neuropharm.2015.04.005

Sakamoto, K., Endo, K., Suzuki, T., Fujimura, K., Kurauchi, Y., Mori, A., et al. (2015). P2X7 receptor antagonists protect against N-methyl-D-aspartic acidinduced neuronal injury in the rat retina. Eur. J Pharmacol. 756, 52-58. doi: 10.1016/j.ejphar.2015.03.008

Salgado-Puga, K., Rodriguez-Colorado, J., Prado-Alcala, R. A., and Pena-Ortega, F. (2017). Subclinical doses of ATP-sensitive potassium channel modulators prevent alterations in memory and synaptic plasticity induced by amyloid- $\beta$. J. Alzheimers Dis. 57, 205-226. doi: 10.3233/JAD- 160543

Salvetti, N. R., Panzani, C. G., Gimeno, E. J., Neme, L. G., Alfaro, N. S., and Ortega, H. H. (2009). An imbalance between apoptosis and proliferation contributes to follicular persistence in polycystic ovaries in rats. Reprod. Biol. Endocrinol. 7:68. doi: $10.1186 / 1477-7827-7-68$

Samuel, S. S., Mani, A., Tachett, B., Desai, M., and Thevananther, S. (2010). P2Y2 purinergic receptor activation is essential for endotoxin-induced acute liver injury in mice. Hepatology 52:608A.

Sanderson, J., Dartt, D. A., Trinkaus-Randall, V., Pintor, J., Civan, M. M., Delamere, N. A., et al. (2014). Purines in the eye: recent evidence for the physiological and pathological role of purines in the RPE, retinal neurons, astrocytes, Muller cells, lens, trabecular meshwork, cornea and lacrimal gland. Exp. Eye Res. 127, 270-279. doi: 10.1016/j.exer.2014.08.009

Santana, P. T., Benjamim, C. F., Martinez, C. G., Kurtenbach, E., Takiya, C. M., and Coutinho-Silva, R. (2015). The P2X7 receptor contributes to the development of the exacerbated inflammatory response associated with sepsis. J. Innate Immun. 7, 417-427. doi: 10.1159/000371388

Santiago, A. R., Baptista, F. I., Santos, P. F., Cristóvão, G., Ambrósio, A. F., Cunha, R. A., et al. (2014). Role of microglia adenosine A2A receptors in retinal and brain neurodegenerative diseases. Mediators Inflamm. 2014:465694. doi: 10.1155/2014/465694

Sarafoff, N., Byrne, R. A., and Sibbing, D. (2012). Clinical use of clopidogrel. Curr. Pharm. Des. 18, 5224-5239. doi: 10.2174/138161212803251853

Sashindranath, M., Dwyer, K. M., Dezfouli, S., Selan, C., Crikis, S., Lu, B., et al. (2017). Development of a novel strategy to target CD39 antithrombotic activity to the endothelial-platelet microenvironment in kidney ischemia-reperfusion injury. Purinergic Signal. 13, 259-265. doi: 10.1007/s11302-017-9558-3

Sauer, A. V., Mrak, E., Hernandez, R. J., Zacchi, E., Cavani, F., Casiraghi, M., et al. (2009). ADA-deficient SCID is associated with a specific microenvironment and bone phenotype characterized by RANKL/OPG imbalance and osteoblast insufficiency. Blood 114, 3216-3226. doi: 10.1182/blood-2009-03-209221

Savio, L. E., Andrade, M. G., De Andrade Mello, P., Santana, P. T., MoreiraSouza, A. C., Kolling, J., et al. (2016). P2X7 receptor signaling contributes to sepsis-associated brain dysfunction. Mol. Neurobiol. 54, 6459-6470. doi: 10.1007/s12035-016-0168-9

Savio, L. E., and Coutinho-Silva, R. (2016). Purinergic signaling in infection and autoimmune disease. Biomed. J. 39, 304-305. doi: 10.1016/j.bj.2016.09.002

Sawynok, J. (2016). Adenosine receptor targets for pain. Neuroscience 338, 1-18. doi: 10.1016/j.neuroscience.2015.10.031

Schepp, C. P., and Reutershan, J. (2008). Bench-to-bedside review: adenosine receptors-promising targets in acute lung injury? Crit. Care 12:226. doi: $10.1186 /$ cc6990
Schneider, G., Glaser, T., Lameu, C., Bdelbaset-Ismail, A., Sellers, Z. P., Moniuszko, M., et al. (2015). Extracellular nucleotides as novel, underappreciated pro-metastatic factors that stimulate purinergic signaling in human lung cancer cells. Mol. Cancer 14:201. doi: 10.1186/s12943-015-0469-z

Schulte am Esch, J., Akyildiz, A., Tustas, R. Y., Ganschow, R., Schmelzle, M., Krieg, A., et al. (2010). ADP-dependent platelet function prior to and in the early course of pediatric liver transplantation and persisting thrombocytopenia are positively correlated with ischemia/reperfusion injury. Transpl. Int. 23, 745-752. doi: 10.1111/j.1432-2277.2010.01054.x

Schulz, N., Liu, K. C., Charbord, J., Mattsson, C. L., Tao, L., Tworus, D., et al. (2016). Critical role for adenosine receptor A2a in $\beta$-cell proliferation. Mol. Metab. 5, 1138-1146. doi: 10.1016/j.molmet.2016.09.006

Sebastián-Serrano, A., Engel, T., De Diego-García, L., Olivos-Oré, L. A., ArribasBlázquez, M., Martínez-Frailes, C., et al. (2016). Neurodevelopmental alterations and seizures developed by mouse model of infantile hypophosphatasia are associated with purinergic signalling deregulation. Hum. Mol. Genet. 25, 4143-4156. doi: 10.1093/hmg/ddw248

Seref-Ferlengez, Z., Maung, S., Schaffler, M. B., Spray, D. C., Suadicani, S. O., and Thi, M. M. (2016). P2X7R-Panx1 complex impairs bone mechanosignaling under high glucose levels associated with Type-1 diabetes. PLOS ONE 11:e0155107. doi: 10.1371/journal.pone.0155107

Shabbir, M., and Burnstock, G. (2009). Purinergic receptor-mediated effects of ATP in urogenital malignant diseases. Int. J. Urol. 16, 143-150. doi: 10.1111/j.14422042.2008.02207.x

Shabbir, M., Ryten, M., Thompson, C. S., Mikhailidis, D. P., and Burnstock, G. (2008). Purinergic receptor-mediated effects of ATP in high-grade bladder cancer. BJU Int. 101, 106-112.

Shaikh, G., and Cronstein, B. (2016). Signaling pathways involving adenosine $\mathrm{A} 2 \mathrm{~A}$ and $\mathrm{A} 2 \mathrm{~B}$ receptors in wound healing and fibrosis. Purinergic Signal. 12, 191-197. doi: 10.1007/s11302-016-9498-3

Shcherbatko, A., Foletti, D., Poulsen, K., Strop, P., Zhu, G., Hasa-Moreno, A., et al. (2016). Modulation of P2X3 and P2X2/3 receptors by monoclonal antibodies. J. Biol. Chem. 291, 12254-12270. doi: 10.1074/jbc.M116.722330

Shibukawa, Y., Sato, M., Kimura, M., Sobhan, U., Shimada, M., Nishiyama, A., et al. (2015). Odontoblasts as sensory receptors: transient receptor potential channels, pannexin-1, and ionotropic ATP receptors mediate intercellular odontoblast-neuron signal transduction. Pflugers. Arch. 467, 843-863. doi: 10.1007/s00424-014-1551-x

Shimizu, Y., and Calvert, J. W. (2016). Recycling KATP channels for cardioprotection. Am. J. Physiol. Heart Circ. Physiol. 310, H1381-H1382. doi: 10.1152/ajpheart.00309.2016

Shishikura, Y., Koarai, A., Sugiura, H., Aizawa, H., Hashimoto, Y., Numakura, T., et al. (2016). Extracellular ATP involves in dsRNA-induced MUC5AC production via P2Y2R in human airway epithelium. Am. J. Respir. Crit. Care Med. 193, A5851.

Sillero, M. A., De Diego, A., Tavares, J. E., Silva, J. A., Pérez-Zúñiga, F. J., and Sillero, A. (2009). Synthesis of ATP derivatives of compounds of the mevalonate pathway (isopentenyl di- and triphosphate; geranyl di- and triphosphate, farnesyl di- and triphosphate, and dimethylallyl diphosphate) catalyzed by T4 RNA ligase, T4 DNA ligase and other ligases Potential relationship with the effect of bisphosphonates on osteoclasts. Biochem. Pharmacol. 78, 335-343. doi: $10.1016 /$ j.bcp.2009.04.028

Silva, C. L. (2016). Purinergic signaling in schistosomal infection. Biomed. J. 39, 316-325. doi: 10.1016/j.bj.2016.06.006

Silva-Ramos, M., Silva, I., Oliveira, J. C., and Correia-De-Sa, P. (2016). Increased urinary adenosine triphosphate in patients with bladder outlet obstruction due to benign prostate hyperplasia. Prostate 76, 1353-1363. doi: 10.1002/pros.23207

Silva-Ramos, M., Silva, I., Oliveira, O., Ferreira, S., Reis, M. J., Oliveira, J. C., et al. (2013). Urinary ATP may be a dynamic biomarker of detrusor overactivity in women with overactive bladder syndrome. PLOS ONE 8:e64696. doi: 10.1371/ journal.pone.0064696

Silveira, G. F., Buffon, A., and Bruno, A. N. (2013). New approaches to thyroid hormones and purinergic signaling. J. Thyroid Res. 2013:434727. doi: 10.1155/ 2013/434727

Silverman, M. H., Strand, V., Markovits, D., Nahir, M., Reitblat, T., Molad, Y., et al. (2008). Clinical evidence for utilization of the $\mathrm{A} 3$ adenosine receptor as a target to treat rheumatoid arthritis: data from a phase II clinical trial. J. Rheumatol. 35, $41-48$. 
Sinadinos, A., Young, C. N., Al-Khalidi, R., Teti, A., Kalinski, P., Mohamad, S., et al. (2015). P2RX7 purinoceptor: a therapeutic target for ameliorating the symptoms of duchenne muscular dystrophy. PLOS Med. 12:e1001888. doi: 10.1371/journal.pmed.1001888

Singh, S., Roy, K. K., Khan, S. R., Kashyap, V. K., Sharma, A., Jaiswal, S., et al. (2015). Novel, potent, orally bioavailable and selective mycobacterial ATP synthase inhibitors that demonstrated activity against both replicating and nonreplicating M. tuberculosis. Bioorg. Med. Chem. 23, 742-752. doi: 10.1016/j.bmc. 2014.12.060

Sivak, K. V., Vasin, A. V., Egorov, V. V., Tsevtkov, V. B., Kuzmich, N. N., Savina, V. A., et al. (2016). Adenosine A2A receptor as a drug target for treatment of sepsis. Mol. Biol. 50, 231-245. doi: 10.7868/S0026898416020233

Sjuve Scott, R., Uvelius, B., and Arner, A. (2004). Changes in intracellular calcium concentration and $\mathrm{P} 2 \mathrm{X} 1$ receptor expression in hypertrophic rat urinary bladder smooth muscle. Neurourol. Urodyn. 23, 361-366. doi: 10.1002/nau. 20047

Slater, M., Danieletto, S., Gidley-Baird, A., Teh, L. C., and Barden, J. A. (2004). Early prostate cancer detected using expression of non-functional cytolytic P2X7 receptors. Histopathology 44, 206-215. doi: 10.1111/j.0309-0167.2004. 01798.x

Smith, C. P., Vemulakonda, V. M., Kiss, S., Boone, T. B., and Somogyi, G. T. (2005). Enhanced ATP release from rat bladder urothelium during chronic bladder inflammation: effect of botulinum toxin A. Neurochem. Int. 47, 291-297. doi: 10.1016/j.neuint.2005.04.021

Smith, S. B., Xu, Z., Novitskaya, T., Zhang, B., Chepurko, E., Pu, X. A., et al. (2016). Impact of cardiac-specific expression of CD39 on myocardial infarct size in mice. Life Sci. 179, 54-59. doi: 10.1016/j.lfs.2016.10.016

Soares, A. F., Diniz, C., and Fresco, P. (2012). A3-adenosine receptor effects on malignant melanoma cells. FEBS J. 279:547.

Soares-Bezerra, R. J., Pinho, R. T., Bisaggio Rda, C., Benevolo-De-Andrade, T. C., and Alves, L. A. (2015). The search for new agonists to P2X7R for clinical use: tuberculosis as a possible target. Cell Physiol. Biochem. 37, 409-418. doi: 10.1159/000430364

Solini, A., Menini, S., Rossi, C., Ricci, C., Santini, E., Blasetti Fantauzzi, C., et al. (2013). The purinergic $2 X 7$ receptor participates in renal inflammation and injury induced by high-fat diet: possible role of NLRP3 inflammasome activation. J. Pathol. 231, 342-353. doi: 10.1002/path.4237

Soma, T., Kobayashi, T., Nakagome, K., and Nagata, M. (2016). Clinical implication of adenosine triphosphate in sputum in asthma. Am. J. Respir. Crit. Care Med. 193, A1394.

Song, H., Han, Y., Pan, C., Deng, X., Dai, W., Hu, L., et al. (2015). Activation of adenosine monophosphate-activated protein kinase suppresses neuroinflammation and ameliorates bone cancer pain: involvement of inhibition on mitogen-activated protein kinase. Anesthesiology 123, 1170-1185. doi: 10.1097/ALN.0000000000000856

Song, S., Jacobson, K. N., Mcdermott, K. M., Reddy, S. P., Cress, A. E., Tang, H., et al. (2016). ATP promotes cell survival via regulation of cytosolic [Ca2+] and Bcl-2/Bax ratio in lung cancer cells. Am. J. Physiol. Cell Physiol. 310, C99-C114. doi: 10.1152/ajpcell.00092.2015

Sousa, J. B., and Diniz, C. (2017). The adenosinergic system as a therapeutic target in the vasculature: new ligands and challenges. Molecules 22:E752. doi: 10.3390/molecules22050752

Spaans, F., Melgert, B. N., Borghuis, T., Klok, P. A., De Vos, P., Bakker, W. W., et al. (2014a). Extracellular adenosine triphosphate affects systemic and kidney immune cell populations in pregnant rats. Am. J. Reprod. Immunol. 72, 305-316. doi: 10.1111/aji.12267

Spaans, F., Melgert, B. N., Chiang, C., Borghuis, T., Klok, P. A., De Vos, P., et al. (2014b). Extracellular ATP decreases trophoblast invasion, spiral artery remodeling and immune cells in the mesometrial triangle in pregnant rats. Placenta 35, 587-595. doi: 10.1016/j.placenta.2014.05.013

Sperlagh, B., and Illes, P. (2014). P2X7 receptor: an emerging target in central nervous system diseases. Trends Pharmacol. Sci. 35, 537-547. doi: 10.1016/j.tips. 2014.08.002

Squadrito, F., Bitto, A., Altavilla, D., Arcoraci, V., De Caridi, G., De Feo, M. E., et al. (2014). The effect of PDRN, an adenosine receptor A2A agonist, on the healing of chronic diabetic foot ulcers: results of a clinical trial. J. Clin. Endocrinol. Metab. 99, E746-E753. doi: 10.1210/jc.2013-3569
Stachon, P., Heidenreich, A., Merz, J., Hoppe, N., Bode, C., Idzko, M., et al. (2016). Deficiency of purinergic receptor P2X7 reduces atherosclerosis in mice. Circulation 134(Suppl._1), A16502.

Stagg, J., Beavis, P. A., Divisekera, U., Liu, M. C., Moller, A., Darcy, P. K., et al. (2012). CD73-deficient mice are resistant to carcinogenesis. Cancer Res. 72, 2190-2196. doi: 10.1158/0008-5472.CAN-12-0420

Stagg, J., Divisekera, U., Mclaughlin, N., Sharkey, J., Pommey, S., Denoyer, D., et al. (2010). Anti-CD73 antibody therapy inhibits breast tumor growth and metastasis. Proc. Natl. Acad. Sci. U.S.A. 107, 1547-1552. doi: 10.1073/pnas. 0908801107

Stamp, L. K., Hazlett, J., Roberts, R. L., Frampton, C., Highton, J., and Hessian, P. A. (2012). Adenosine receptor expression in rheumatoid synovium: a basis for methotrexate action. Arthritis Res. Ther. 14:R138. doi: 10.1186/ar3871

Steculorum, S. M., Timper, K., Engström Ruud, L., Evers, N., Paeger, L., Bremser, S., et al. (2017). Inhibition of P2Y6 signaling in AgRP neurons reduces food intake and improves systemic insulin sensitivity in obesity. Cell Rep. 18, 1587-1597. doi: 10.1016/j.celrep.2017.01.047

Stemmer, S., Silverman, M. H., Kerns, W. D., Bar-Yehuda, S., Fishman, S., Harpaz, Z., et al. (2010). Phase $1 / 2$ trial of CF102, a selective A3 adenosine receptor (A3AR) agonist, in patients with hepatocellular carcinoma (HCC). Eur. J. Cancer 8:122. doi: 10.1634/theoncologist.2012-0211

Sterle, I., Zupancic, D., and Romih, R. (2014). Correlation between urothelial differentiation and sensory proteins P2X3, P2X5, TRPV1, and TRPV4 in normal urothelium and papillary carcinoma of human bladder. Biomed. Res. Int. 2014:805236. doi: 10.1155/2014/805236

Stoll, M., Kim, Y. O., Bebich, B., Robson, S. C., and Schuppan, D. (2012). The selective adenosine 2B receptor antagonist MRS1754 mitigates hepatic collagen deposition during fibrosis progression and induces mild fibrosis regression. Gastroenterology 142, S974-S975. doi: 10.1016/S0016-5085(12)63778-0

Straub, R. H., Rauch, L., Fassold, A., Lowin, T., and Pongratz, G. (2008). Neuronally released sympathetic neurotransmitters stimulate splenic interferon- $\gamma$ secretion from T cells in early type II collagen-induced arthritis. Arthritis Rheum. 58, 3450-3460. doi: 10.1002/art.24030

Suadicani, S. O., Urban-Maldonado, M., Tar, M. T., Melman, A., and Spray, D. C. (2009). Effects of ageing and streptozotocin-induced diabetes on connexin 43 and P2 purinoceptor expression in the rat corpora cavernosa and urinary bladder. BJU Int. 103, 1686-1693. doi: 10.1111/j.1464-410X.2008.08337.x

Subramanian, M., Kini, R., Madasu, M., Ohta, A., Nowak, M., Exley, M., et al. (2014). Extracellular adenosine controls NKT-cell-dependent hepatitis induction. Eur. J. Immunol. 44, 1119-1129. doi: 10.1002/eji.201343866

Sugiyama, T. (2014). Role of P2X7 receptors in the development of diabetic retinopathy. World J. Diabetes 5, 141-145. doi: 10.4239/wjd.v5.i2.141

Sun, H. S., Xu, B., Chen, W., Xiao, A., Turlova, E., Alibraham, A., et al. (2015). Neuronal KATP channels mediate hypoxic preconditioning and reduce subsequent neonatal hypoxic-ischemic brain injury. Exp. Neurol. 263, 161-171. doi: 10.1016/j.expneurol.2014.10.003

Sun, L., Gao, J., Zhao, M., Cui, J., Li, Y., Yang, X., et al. (2015). A novel cognitive impairment mechanism that astrocytic p-connexin 43 promotes neuronic autophagy via activation of P2X7R and down-regulation of GLT-1 expression in the hippocampus following traumatic brain injury in rats. Behav. Brain Res. 291, 315-324. doi: 10.1016/j.bbr.2015.05.049

Sun, X., Han, L., Seth, P., Bian, S., Li, L., Csizmadia, E., et al. (2013). Disordered purinergic signaling and abnormal cellular metabolism are associated with development of liver cancer in Cd39/ENTPD1 null mice. Hepatology 57, 205-216. doi: 10.1002/hep.25989

Sun, X., Imai, M., Nowak-Machen, M., Guckelberger, O., Enjyoji, K., Wu, Y., et al. (2011). Liver damage and systemic inflammatory responses are exacerbated by the genetic deletion of CD39 in total hepatic ischemia. Purinergic Signal. 7, 427-434. doi: 10.1007/s11302-011-9239-6

Sunggip, C., Nishimura, A., Shimoda, K., Numaga-Tomita, T., Tsuda, M., and Nishida, M. (2017). Purinergic P2Y6 receptors: A new therapeutic target of agedependent hypertension. Pharmacol. Res. 120, 51-59. doi: 10.1016/j.phrs.2017. 03.013

Suriyaphol, G., Sarikaputi, M., and Suriyaphol, P. (2009). Differential responses of cells from human skin keratinocyte and bovine mammary epithelium to attack by pore-forming Staphylococcus aureus $\alpha$-toxin. Comp. Immunol. Microbiol. Infect. Dis. 32, 491-502. doi: 10.1016/j.cimid.2008.07.002 
Swennen, E. L., Dagnelie, P. C., Van Den Beucken, T., and Bast, A. (2008). Radioprotective effects of ATP in human blood ex vivo. Biochem. Biophys. Res. Commun. 367, 383-387. doi: 10.1016/j.bbrc.2007.12.125

Swiatkowski, P., Murugan, M., Eyo, U. B., Wang, Y., Rangaraju, S., Oh, S. B., et al. (2016). Activation of microglial P2Y12 receptor is required for outward potassium currents in response to neuronal injury. Neuroscience 318, 22-33. doi: 10.1016/j.neuroscience.2016.01.008

Szkudelski, T. (2007). Intracellular mediators in regulation of leptin secretion from adipocytes. Physiol. Res. 56, 503-512.

Szuster-Ciesielska, A., Sztanke, K., and Kandefer-Szerszen, M. (2012). A novel fused 1,2,4-triazine aryl derivative as antioxidant and nonselective antagonist of adenosine A2A receptors in ethanol-activated liver stellate cells. Chem. Biol. Interact. 195, 18-24. doi: 10.1016/j.cbi.2011.10.004

Tai, Y. H., Cheng, P. Y., Tsai, R. Y., Chen, Y. F., and Wong, C. S. (2010). Purinergic $\mathrm{P} 2 \mathrm{X}$ receptor regulates $\mathrm{N}$-methyl-D-aspartate receptor expression and synaptic excitatory amino acid concentration in morphine-tolerant rats. Anesthesiology 113, 1163-1175. doi: 10.1097/ALN.0b013e3181f11aa2

Tak, E., Jun, D. Y., Kim, S. H., Park, G. C., Lee, J., Hwang, S., et al. (2016). Upregulation of P2Y2 nucleotide receptor in human hepatocellular carcinoma cells. J. Int. Med. Res. 44, 1234-1247. doi: 10.1177/0300060516662135

Tak, E., Ridyard, D., Kim, J. H., Zimmerman, M., Werner, T., Wang, X. X., et al. (2014). CD73-dependent generation of adenosine and endothelial Adora2b signaling attenuate diabetic nephropathy. J. Am. Soc. Nephrol. 25, 547-563. doi: 10.1681/ASN.2012101014

Takai, E., Tsukimoto, M., Harada, H., and Kojima, S. (2011). Involvement of P2Y6 receptor in p38 MAPK-mediated COX-2 expression in response to UVB irradiation of human keratinocytes. Radiat. Res. 175, 358-366. doi: 10.1667/ RR2375.1

Takai, E., Tsukimoto, M., Harada, H., and Kojima, S. (2014). Autocrine signaling via release of ATP and activation of $\mathrm{P} 2 \mathrm{X} 7$ receptor influences motile activity of human lung cancer cells. Purinergic Signal. 10, 487-497. doi: 10.1007/s11302014-9411-x

Takenouchi, T., Tsukimoto, M., Hashimoto, M., and Kitani, H. (2014). Inflammasome activation by danger signals: extracellular ATP and $\mathrm{pH}$. Inflammasome 1, 76-80. doi: 10.2478/infl-2014-0008

Tanaka, S., Kudo, H., Asari, T., Ono, A., Motomura, S., Toh, S., et al. (2011). P2Y1 transient overexpression induced mineralization in spinal ligament cells derived from patients with ossification of the posterior longitudinal ligament of the cervical spine. Calcif. Tissue Int. 88, 263-271. doi: 10.1007/s00223-010-9456-y

Tang, J., Li, M. P., Zhou, H. H., and Chen, X. P. (2015). Platelet inhibition agents: current and future P2Y12 receptor antagonists. Curr. Vasc. Pharmacol. 13, 566-577. doi: 10.2174/1570161112666141127162209

Tang, L. M., Zhu, J. F., Wang, F., Qian, J., Zhu, J., Mo, Q., et al. (2010). Activation of adenosine A2A receptor attenuates inflammatory response in a rat model of small-for-size liver transplantation. Transplant. Proc. 42, 1915-1920. doi: 10.1016/j.transproceed.2010.02.084

Tantry, S. J., Markad, S. D., Shinde, V., Bhat, J., Balakrishnan, G., Gupta, A. K., et al. (2017). Discovery of imidazo[1,2-a]pyridine ethers and squaramides as selective and potent inhibitors of mycobacterial adenosine triphosphate (ATP) synthesis. J. Med. Chem. 60, 1379-1399. doi: 10.1021/acs.jmedchem.6b01358

Tao, Y., and Liang, G. (2015). Efficacy of adenosine A2A receptor antagonist istradefylline as augmentation for Parkinson's disease: a meta-analysis of randomized controlled trials. Cell Biochem. Biophys. 71, 57-62. doi: 10.1007/ s12013-014-0162-7

Taskiran, E., Erbas, O., Yigittürk, G., Meral, A., Akar, H., and Taskiran, D. (2016). Exogenously administered adenosine attenuates renal damage in streptozotocin-induced diabetic rats. Ren. Fail. 38, 1276-1282. doi: 10.1080/ 0886022X.2016.1207054

Taylor, J. M., and Han, Z. (2010). Purinergic receptor functionality is necessary for infection of human hepatocytes by hepatitis delta virus and hepatitis B virus. PLOS ONE 5:e15784. doi: 10.1371/journal.pone.0015784

Taylor, S. R. J., Turner, C. M., Elliott, J. I., Hewitt, R., Pickering, M., Cook, H. T., et al. (2009). P2X7-deficiency ameliorates accelerated nephrotoxic nephritis in mice. J. Am. Soc. Nephrol. 20, 1275-1281. doi: 10.1681/ASN.2008060559

Teixeira, J. M., Bobinski, F., Parada, C. A., Sluka, K. A., and Tambeli, C. H. (2016). P2X3 and P2X2/3 receptors play a crucial role in articular hyperalgesia development through inflammatory mechanisms in the knee joint experimental synovitis. Mol. Neurobiol. doi: 10.1007/s12035-016-0146-2 [Epub ahead of print].

Teramachi, J., Kukita, A., Li, Y. J., Ushijima, Y., Ohkuma, H., Wada, N., et al. (2011). Adenosine abolishes MTX-induced suppression of osteoclastogenesis and inflammatory bone destruction in adjuvant-induced arthritis. Lab. Invest. 91, 719-731. doi: 10.1038/labinvest.2011.9

Thevananther, S., Sun, H., Hernandez, A., Awad, S. S., and Karpen, S. J. (2008). Impaired hepatocellular proliferation in P2Y2 purinergic receptor knockout mice: mitogenic role of extracellular ATP. Hepatology 44:206A.

Thevananther, S., Sun, H., Li, D., Arjunan, V., Awad, S. S., Wyllie, S., et al. (2004). Extracellular ATP activates c-jun N-terminal kinase signaling and cell cycle progression in hepatocytes. Hepatology 39, 393-402. doi: 10.1002/hep.20075

Tian, T., Zhou, Y., Feng, X., Ye, S., Wang, H., Wu, W., et al. (2016). MicroRNA16 is putatively involved in the NF- $\mathrm{B}$ pathway regulation in ulcerative colitis through adenosine A2a receptor (A2aAR) mRNA targeting. Sci. Rep. 6:30824. doi: $10.1038 /$ srep30824

Tian, Y., Marshall, M., French, B. A., Linden, J., and Yang, Z. (2015). The infarctsparing effect of IB-MECA against myocardial ischemia/reperfusion injury in mice is mediated by sequential activation of adenosine $\mathrm{A} 3$ and $\mathrm{A} 2 \mathrm{~A}$ receptors. Basic Res. Cardiol. 110:16. doi: 10.1007/s00395-015-0473-X

Tian, Y., Tian, X., Han, X., Chen, Y., Song, C. Y., Zhang, Y. B., et al. (2016). Expression of ATP binding cassette E1 enhances viability and invasiveness of lung adenocarcinoma cells in vitro. Mol. Med. Rep. 14, 1345-1350. doi: 10.3892/ mmr.2016.5388

Torres, A., Vargas, Y., Uribe, D., Jaramillo, C., Gleisner, A., Salazar-Onfray, F., et al. (2016). Adenosine A3 receptor elicits chemoresistance mediated by multiple resistance-associated protein-1 in human glioblastoma stem-like cells. Oncotarget 7, 67373-67386. doi: 10.18632/oncotarget.12033

Torres, B. T., Jimenez, D. A., and Budsberg, S. C. (2016). Elevated synovial fluid concentration of adenosine triphosphate in dogs with osteoarthritis or sodium urate-induced synovitis of the stifle. Vet. Comp. Orthop. Traumatol. 29, 344-346. doi: 10.3415/VCOT-15-06-0111

Torres-Fuentes, J. L., Rios, M., and Moreno, R. D. (2015). Involvement of a P2X7 receptor in the acrosome reaction induced by ATP in rat spermatozoa. J. Cell. Physiol. 230, 3068-3075. doi: 10.1002/jcp.25044

Truong, L. D., Trostel, J., Mcmahan, R., Chen, J. F., and Garcia, G. E. (2016). Macrophage A2A adenosine receptors are essential to protect from progressive kidney injury. Am. J. Pathol. 186, 2601-2613. doi: 10.1016/j.ajpath.2016.06.017

Tsuda, M. (2016). Microglia in the spinal cord and neuropathic pain. J. Diabetes Investig. 7, 17-26. doi: 10.1111/jdi.12379

Tsuda, M. (2017). P2 receptors, microglial cytokines and chemokines, and neuropathic pain. J. Neurosci. Res. 95, 1319-1329. doi: 10.1002/jnr.23816

Tsuda, M., and Inoue, K. (2016). Neuron-microglia interaction by purinergic signaling in neuropathic pain following neurodegeneration. Neuropharmacology 104, 76-81. doi: 10.1016/j.neuropharm.2015.08.042

Tsuda, M., Koga, K., Chen, T., and Zhuo, M. (2017). Neuronal and microglial mechanisms for neuropathic pain in the spinal dorsal horn and anterior cingulate cortex. J. Neurochem. 141, 486-498. doi: 10.1111/jnc.14001

Tu, G., Zou, L., Liu, S., Wu, B., Lv, Q., Wang, S., et al. (2016). Long noncoding NONRATT021972 siRNA normalized abnormal sympathetic activity mediated by the upregulation of $\mathrm{P} 2 \mathrm{X} 7$ receptor in superior cervical ganglia after myocardial ischemia. Purinergic Signal. 12, 521-535. doi: 10.1007/s11302-0169518-3

Turcin, A., Dolzan, V., Porcelli, S., Serretti, A., and Plesnicar, B. K. (2016). Adenosine hypothesis of antipsychotic drugs revisited: pharmacogenomics variation in nonacute schizophrenia. OMICS 20, 283-289. doi: 10.1089/omi. 2016.0003

Uchida, S., Soshiroda, K., Okita, E., Kawai-Uchida, M., Mori, A., Jenner, P., et al. (2015). The adenosine A2A receptor antagonist, istradefylline enhances the anti-parkinsonian activity of low doses of dopamine agonists in MPTP-treated common marmosets. Eur. J. Pharmacol. 747, 160-165. doi: 10.1016/j.ejphar. 2014.11.038

Uluçkan, Ö., Eagleton, M. C., Floyd, D. H., Morgan, E. A., Hirbe, A. C., Kramer, M., et al. (2008). APT102, a novel adpase, cooperates with aspirin to disrupt bone metastasis in mice. J. Cell. Biochem 104, 1311-1323. doi: 10.1002/jcb.21709

Ursu, D., Ebert, P., Langron, E., Ruble, C., Munsie, L., Zou, W., et al. (2014). Gain and loss of function of P2X7 receptors: mechanisms, pharmacology and 
relevance to diabetic neuropathic pain. Mol. Pain 10:37. doi: 10.1186/17448069-10-37

Valladares, D., Altamirano, F., Henríquez-Olguín, C., Díaz-Vegas, A., Intriago, G., Contreras-Ferrat, A., et al. (2014). ATP signaling complex is altered in muscular dystrophy and was partly recovered after nifedipine treatment. FASEB J. 28, 762.3 .

Vandenbeuch, A., Anderson, C. B., Parnes, J., Enjyoji, K., Robson, S. C., Finger, T. E., et al. (2013). Role of the ectonucleotidase NTPDase2 in taste bud function. Proc. Natl. Acad. Sci. U.S.A. 110, 14789-14794. doi: 10.1073/pnas.130946 8110

Vandenbeuch, A., Larson, E. D., Anderson, C. B., Smith, S. A., Ford, A. P., Finger, T. E., et al. (2015). Postsynaptic P2X3-containing receptors in gustatory nerve fibres mediate responses to all taste qualities in mice. J. Physiol. 593, 1113-1125. doi: 10.1113/jphysiol.2014.281014

Vanderstocken, G., Van De Paar, E., Robaye, B., Di Pietrantonio, L., Bondue, B., Boeynaems, J. M., et al. (2012). Protective role of P2Y2 receptor against lung infection induced by pneumonia virus of mice. PLOS ONE 7:e50385. doi: 10.1371/journal.pone.0050385

Varani, K., Padovan, M., Govoni, M., Vincenzi, F., Trotta, F., and Borea, P. A. (2010a). The role of adenosine receptors in rheumatoid arthritis. Autoimmun. Rev. 10, 61-64. doi: 10.1016/j.autrev.2010.07.019

Varani, K., Vincenzi, F., Targa, M., Paradiso, B., Parrilli, A., Fini, M., et al. (2013). The stimulation of $\mathrm{A} 3$ adenosine receptors reduces bone-residing breast cancer in a rat preclinical model. Eur. J. Cancer 49, 482-491. doi: 10.1016/j.ejca.2012. 06.005

Varani, K., Vincenzi, F., Tosi, A., Targa, M., Masieri, F. F., Ongaro, A., et al. (2010b). Expression and functional role of adenosine receptors in regulating inflammatory responses in human synoviocytes. Br. J. Pharmacol. 160, 101-115. doi: 10.1111/j.1476-5381.2010.00667.x

Vaughn, B. P., Robson, S. C., and Longhi, M. S. (2014). Purinergic signaling in liver disease. Dig. Dis. 32, 516-524. doi: 10.1159/000360498

Vazquez-Cuevas, F. G., Martinez-Ramirez, A. S., Robles-Martinez, L., Garay, E., Garcia-Carranca, A., Perez-Montiel, D., et al. (2014). Paracrine stimulation of P2X7 receptor by ATP activates a proliferative pathway in ovarian carcinoma cells. J. Cell. Biochem. 115, 1955-1966. doi: 10.1002/jcb.24867

Vergani, A., Fotino, C., D’addio, F., Tezza, S., Podetta, M., Gatti, F., et al. (2013). Effect of the purinergic inhibitor oxidized ATP in a model of islet allograft rejection. Diabetes Metab. Res. Rev. 62, 1665-1675. doi: 10.2337/db120242

Veselá, R., Aronsson, P., Andersson, M., Wsol, V., and Tobin, G. (2012a). The potential of non-adrenergic, non-cholinergic targets in the treatment of interstitial cystitis/painful bladder syndrome. J. Physiol. Pharmacol. 63, 209-216.

Veselá, R., Aronsson, P., and Tobin, G. (2011). Functional and morphological examinations of P1Al purinoceptors in the normal and inflamed urinary bladder of the rat. Auton. Neurosci. 159, 26-31. doi: 10.1016/j.autneu.2010. 07.008

Veselá, R., Asklund, H., Aronsson, P., Johnsson, M., Wsol, V., Andersson, M., et al. (2012b). Coupled nitric oxide and autonomic receptor functional responses in the normal and inflamed urinary bladder of the rat. Physiol. Res. 61, 371-380.

Vieira, F. S., Nanini, H. F., Takiya, C. M., and Coutinho-Silva, R. (2016). P2X7 receptor knockout prevents streptozotocin-induced type 1 diabetes in mice. Mol. Cell. Endocrinol. 419, 148-157. doi: 10.1016/j.mce.2015.10.008

Vincent, I. S., and Okusa, M. D. (2015). Adenosine 2A receptors in acute kidney injury. Acta Physiol. 214, 303-310. doi: 10.1111/apha.12508

Vincenzi, F., Padovan, M., Targa, M., Corciulo, C., Giacuzzo, S., Merighi, S., et al. (2013). A2A adenosine receptors are differentially modulated by pharmacological treatments in rheumatoid arthritis patients and their stimulation ameliorates adjuvant-induced arthritis in rats. PLOS ONE 8:e54195. doi: 10.1371/journal.pone.0054195

Vincenzi, F., Ravani, A., Pasquini, S., Merighi, S., Gessi, S., Romagnoli, R., et al. (2016). Positive allosteric modulation of Al adenosine receptors as a novel and promising therapeutic strategy for anxiety. Neuropharmacology 111, 283-292. doi: 10.1016/j.neuropharm.2016.09.015

Vindeirinho, J., Santiago, A. R., Cavadas, C., Ambrósio, A. F., and Santos, P. F. (2016). The adenosinergic system in diabetic retinopathy. J. Diabetes Res. 2016:4270301. doi: 10.1155/2016/4270301
Vlajkovic, S. M., Ambepitiya, K., Barclay, M., Boison, D., Housley, G. D., and Thorne, P. R. (2017). Adenosine receptors regulate susceptibility to noiseinduced neural injury in the mouse cochlea and hearing loss. Hear. Res. 345, 43-51. doi: 10.1016/j.heares.2016.12.015

Vlaskovska, M., Kasakov, L., Rong, W., Bodin, P., Bardini, M., Cockayne, D. A., et al. (2001). P2X3 knockout mice reveal a major sensory role for urothelially released ATP. J. Neurosci. 21, 5670-5677.

Völkl, T., Ogilvie, A., Neuhuber, W., and Ogilvie, A. (2008). Cell death induced by uridine $5^{\prime}$-triphosphate (UTP) in contrast to adenosine $5^{\prime}$-triphosphate (ATP) in human epidermoid carcinoma cells (A-431). Cell Physiol. Biochem. 22, 441-454. doi: 10.1159/000185491

Volonté, C., Apolloni, S., Parisi, C., and Amadio, S. (2016). Purinergic contribution to amyotrophic lateral sclerosis. Neuropharmacology 104, 180-193. doi: 10.1016/j.neuropharm.2015.10.026

von Versen-Höynck, F., Rajakumar, A., Bainbridge, S. A., Gallaher, M. J., Roberts, J. M., and Powers, R. W. (2009). Human placental adenosine receptor expression is elevated in preeclampsia and hypoxia increases expression of the A2A receptor. Placenta 30, 434-442. doi: 10.1016/j.placenta.2009.02.004

Vonend, O., Turner, C., Chan, C. M., Loesch, A., Dell'anna, G. C., Srai, S. K., et al. (2004). Glomerular expression of the ATP-sensitive P2X7 receptor in diabetic and hypertensive rat models. Kidney Int. 66, 157-166. doi: 10.1111/j.1523-1755. 2004.00717.x

Voors, A. A., Dungen, H. D., Senni, M., Nodari, S., Agostoni, P., Ponikowski, P., et al. (2017). Safety and tolerability of neladenoson bialanate, a novel oral partial adenosine A1 receptor agonist, in patients with chronic heart failure. J. Clin. Pharmacol. 57, 440-451. doi: 10.1002/jcph.828

Vorovenci, R. J., and Antonini, A. (2015). The efficacy of oral adenosine A2A antagonist istradefylline for the treatment of moderate to severe Parkinson's disease. Expert Rev. Neurother. 15, 1383-1390. doi: 10.1586/14737175.2015. 1113131

Waeber, C., and Moskowitz, M. A. (2003). Therapeutic implications of central and peripheral neurologic mechanisms in migraine. Neurology 61, S9-S20. doi: 10.1212/WNL.61.8_suppl_4.S9

Walsh, C. A., Cheng, Y., Mansfield, K. J., Parkin, K., Mukerjee, C., and Moore, K. H. (2013). Decreased intravesical adenosine triphosphate in patients with refractory detrusor overactivity and bacteriuria. J. Urol. 189, 1383-1387. doi: 10.1016/j.juro.2012.10.003

Wan, H. X., Hu, J. H., Xie, R., Yang, S. M., and Dong, H. (2016). Important roles of $\mathrm{P} 2 \mathrm{Y}$ receptors in the inflammation and cancer of digestive system. Oncotarget 7, 28736-28747. doi: 10.18632/oncotarget.7518

Wan, P., Liu, X., Xiong, Y., Ren, Y., Chen, J., Lu, N., et al. (2016). Extracellular ATP mediates inflammatory responses in colitis via P2 x 7 receptor signaling. Sci. Rep. 6:19108. doi: 10.1038/srep19108

Wang, C., Hu, S. M., Xie, H., Qiao, S. G., Liu, H., and Liu, C. F. (2015). Role of mitochondrial ATP-sensitive potassium channel-mediated PKC- $\varepsilon$ in delayed protection against myocardial ischemia/reperfusion injury in isolated hearts of sevoflurane-preconditioned rats. Braz. J. Med. Biol. Res. 48, 528-536. doi: 10.1590/1414-431X20143876

Wang, L., Wan, H., Tang, W., Ni, Y., Hou, X., Pan, L., et al. (2016). Critical roles of adenosine A2A receptor in regulating the balance of Treg/Th17cells in allergic asthma. Clin. Respir. J. doi: 10.1111/cri.12503 [Epub ahead of print].

Wang, N., Rumney, R. M., Yang, L., Robaye, B., Boeynaems, J. M., Skerry, T. M., et al. (2013). The P2Y13 receptor regulates extracellular ATP metabolism and the osteogenic response to mechanical loading. J. Bone Miner. Res. 28, 1446-1456. doi: 10.1002/jbmr.1877

Wang, X., Arcuino, G., Takano, T., Lin, J., Peng, W. G., Wan, P., et al. (2004). P2X7 receptor inhibition improves recovery after spinal cord injury. Nat. Med. 10, 821-827. doi: $10.1038 / \mathrm{nm} 1082$

Wang, X. H., Xie, X., Luo, X. G., Shang, H., and He, Z. Y. (2017). Inhibiting purinergic P2X7 receptors with the antagonist brilliant blue $\mathrm{G}$ is neuroprotective in an intranigral lipopolysaccharide animal model of Parkinson's disease. Mol. Med. Rep. 15, 768-776. doi: 10.3892/mmr.2016.6070

Wang, Z., Cristofaro, V., Cheng, Z., Xiao, X., Ge, R., Sullivan, M., et al. (2013). Urothelium-released ATP contributes to bladder dysfunction in type 2 diabetes. J. Urol. 189, e116. doi: 10.1016/j.juro.2013.02.1668

Wei, C. J., Augusto, E., Gomes, C. A., Singer, P., Wang, Y., Boison, D., et al. (2014). Regulation of fear responses by striatal and extrastriatal adenosine A2A 
receptors in forebrain. Biol. Psychiatry 75, 855-863. doi: 10.1016/j.biopsych. 2013.05.003

Wei, Q., Costanzi, S., Liu, Q. Z., Gao, Z. G., and Jacobson, K. A. (2011). Activation of the P2Y1 receptor induces apoptosis and inhibits proliferation of prostate cancer cells. Biochem. Pharmacol. 82, 418-425. doi: 10.1016/j.bcp.2011.05.013

Wen, J., Wang, B., Du, C., Xu, G., Zhang, Z., Li, Y., et al. (2015). A2B adenosine receptor agonist improves erectile function in diabetic rats. Tohoku J. Exp. Med. 237, 141-148. doi: 10.1620/tjem.237.141

Wen, J., and Xia, Y. (2012). Adenosine signaling: good or bad in erectile function? Arterioscler. Thromb. Vasc. Biol. 32, 845-850. doi: 10.1161/ATVBAHA.111. 226803

Wesselius, A., Bours, M. J., Jørgensen, N. R., Wiley, J., Gu, B., Van Helden, S., et al. (2013). Non-synonymous polymorphisms in the P2RX4 are related to bone mineral density and osteoporosis risk in a cohort of Dutch fracture patients. Purinergic Signal. 9, 123-130. doi: 10.1007/s11302-012-9337-0

Wessels, J. A., Kooloos, W. M., De Jonge, R., De Vries-Bouwstra, J. K., Allaart, C. F., Linssen, A., et al. (2006). Relationship between genetic variants in the adenosine pathway and outcome of methotrexate treatment in patients with recent-onset rheumatoid arthritis. Arthritis Rheum. 54, 2830-2839. doi: 10.1002/art.22032

Wettstein, M. S., Buser, L., Hermanns, T., Roudnicky, F., Eberli, D., Baumeister, P., et al. (2015). CD73 predicts favorable prognosis in patients with nonmuscleinvasive urothelial bladder cancer. Dis. Markers 2015:785461. doi: 10.1155/ 2015/785461

White, C. W., Choong, Y. T., Short, J. L., Exintaris, B., Malone, D. T., Allen, A. M., et al. (2013). Male contraception via simultaneous knockout of $\alpha 1 \mathrm{~A}-$ adrenoceptors and P2X1-purinoceptors in mice. Proc. Natl. Acad. Sci U.S.A. 110, 20825-20830. doi: 10.1073/pnas.1318624110

White, N., Butler, P. E. M., and Burnstock, G. (2005). Human melanomas express functional P2X7 receptors. Cell Tissue Res. 321, 411-418. doi: 10.1007/s00441005-1149-x

White, N., Knight, G. E., Butler, P. E. M., and Burnstock, G. (2009). An in vivo model of melanoma: treatment with ATP. Purinergic Signal. 5, 327-333. doi: 10.1007/s11302-009-9156-0

Wiesler, B., Zech, A., and Idzko, M. (2016). The purinergic receptor P2X4 mediates acute airway inflammation by regulating dendritic cell function. Am. J. Respir. Crit. Care Med. 193, A7543.

Wille, A., Amort, T., Singewald, N., Sartori, S. B., and Lusser, A. (2016). Dysregulation of select ATP-dependent chromatin remodeling factors in high trait anxiety. Behav. Brain Res. 311, 141-146. doi: 10.1016/j.bbr.2016.05.036

Winerdal, M., Winerdal, M. E., Wang, Y. Q., Fredholm, B. B., Winqvist, O., and Ådén, U. (2016). Adenosine A1 receptors contribute to immune regulation after neonatal hypoxic ischemic brain injury. Purinergic Signal. 12, 89-101. doi: 10.1007/s11302-015-9482-3

Wirsdörfer, F., De Leve, S., Cappuccini, F., Eldh, T., Meyer, A. V., Gau, E., et al. (2016). Extracellular adenosine production by ecto-5' - nucleotidase (CD73) enhances radiation-induced lung fibrosis. Cancer Res. 76, 3045-3056. doi: 10.1158/0008-5472.CAN-15-2310

Wojcik, M., Zieleniak, A., Mac-Marcjanek, K., Wozniak, L. A., and Cypryk, K. (2014). The elevated gene expression level of the A2B adenosine receptor is associated with hyperglycemia in women with gestational diabetes mellitus. Diabetes Metab. Res. Rev. 30, 42-53. doi: 10.1002/dmrr.2446

Woods, L. T., Ajit, D., Camden, J. M., Erb, L., and Weisman, G. A. (2016). Purinergic receptors as potential therapeutic targets in Alzheimer's disease. Neuropharmacology 104, 169-179. doi: 10.1016/j.neuropharm.2015.10.031

Wright, A., Mahaut-Smith, M., Symon, F., Sylvius, N., Ran, S., Bafadhel, M., et al. (2016). Impaired P2X1 receptor-mediated adhesion in eosinophils from asthmatic patients. J. Immunol. 196, 4877-4884. doi: 10.4049/jimmunol. 1501585

Wright, S. R., Zanos, P., Georgiou, P., Yoo, J. H., Ledent, C., Hourani, S. M., et al. (2016). A critical role of striatal A2A R-mGlu R interactions in modulating the psychomotor and drug-seeking effects of methamphetamine. Addict. Biol. 21, 811-825. doi: 10.1111/adb.12259

Wu, B., Zhang, C., Zou, L., Ma, Y., Huang, K., Lv, Q., et al. (2016). LncRNA uc. $48+$ siRNA improved diabetic sympathetic neuropathy in type 2 diabetic rats mediated by P2X7 receptor in SCG. Auton. Neurosci. 197, 14-18. doi: 10.1016/j.autneu.2016.04.001

Wu, H., Nie, Y., Xiong, H., Liu, S., Li, G., Huang, A., et al. (2015). P2X7 receptor expression in peripheral blood monocytes is correlated with plasma
C-reactive protein and cytokine levels in patients with type 2 diabetes mellitus: a preliminary report. Inflammation 38, 2076-2081. doi: 10.1007/s10753-0150189-y

Wu, J. X., Xu, M. Y., Miao, X. R., Lu, Z. J., Yuan, X. M., Li, X. Q., et al. (2012). Functional up-regulation of $\mathrm{P} 2 \mathrm{X} 3$ receptors in dorsal root ganglion in a rat model of bone cancer pain. Eur. J. Pain 16, 1378-1388. doi: 10.1002/j.15322149.2012.00149.x

Wu, M., Sahbaie, P., Zheng, M., Lobato, R., Boison, D., Clark, J. D., et al. (2013). Opiate-induced changes in brain adenosine levels and narcotic drug responses. Neuroscience 228, 235-242. doi: 10.1016/j.neuroscience.2012.10.031

Wu, W., He, Y., Feng, X., Ye, S., Wang, H., Tan, W., et al. (2017). MicroRNA-206 is involved in the pathogenesis of ulcerative colitis via regulation of adenosine A3 receptor. Oncotarget 8, 705-721. doi: 10.18632/oncotarget.13525

Xia, H., Zhang, D., Yang, S., Wang, Y., Xu, L., Wu, J., et al. (2014). Role of ATPsensitive potassium channels in modulating nociception in rat model of bone cancer pain. Brain Res. 1554, 29-35. doi: 10.1016/j.brainres.2014.01.032

Xia, J., Yu, X., Tang, L., Li, G., and He, T. (2015). P2X7 receptor stimulates breast cancer cell invasion and migration via the AKT pathway. Oncol. Rep. 34, 103-110. doi: 10.3892/or.2015.3979

Xiang, H. J., Liu, Z. C., Wang, D. S., Chen, Y., Yang, Y. L., and Dou, K. F. (2006). Adenosine A2b receptor is highly expressed in human hepatocellular carcinoma. Hepatol. Res. 36, 56-60. doi: 10.1016/j.hepres.2006.06.008

Xiao, F., Waldrop, S. L., Khimji, A. K., and Kilic, G. (2012). Pannexin1 contributes to pathophysiological ATP release in lipoapoptosis induced by saturated free fatty acids in liver cells. Am. J. Physiol. Cell Physiol. 303, C1034-C1044. doi: 10.1152/ajpcell.00175.2012

Xiao, H., Si, L. Y., Liu, W., Li, N., Meng, G., Yang, N., et al. (2013). The effects of adenosine A2A receptor knockout on renal interstitial fibrosis in a mouse model of unilateral ureteral obstruction. Acta Histochem. 115, 315-319. doi: 10.1016/j.acthis.2012.09.002

Xie, R., Xu, J., Wen, G., Jin, H., Liu, X., Yang, Y., et al. (2014). The P2Y2 nucleotide receptor mediates the proliferation and migration of human hepatocellular carcinoma cells induced by ATP. J. Biol. Chem. 289, 19137-19149. doi: 10.1074/ jbc.M113.540047

Xie, Y., Williams, C. D., Mcgill, M. R., Lebofsky, M., Ramachandran, A., and Jaeschke, H. (2013). Purinergic receptor antagonist A438079 protects against acetaminophen-induced liver injury by inhibiting P450 isoenzymes, not by inflammasome activation. Toxicol. Sci. 131, 325-335. doi: 10.1093/toxsci/kfs283

Xu, J., Chu, K. L., Brederson, J. D., Jarvis, M. F., and Mcgaraughty, S. (2012). Spontaneous firing and evoked responses of spinal nociceptive neurons are attenuated by blockade of $\mathrm{P} 2 \mathrm{X} 3$ and $\mathrm{P} 2 \mathrm{X} 2 / 3$ receptors in inflamed rats. J. Neurosci. Res. 90, 1597-1606. doi: 10.1002/jnr.23042

Xu, P., Xu, Y., Hu, B., Wang, J., Pan, R., Murugan, M., et al. (2015). Extracellular ATP enhances radiation-induced brain injury through microglial activation and paracrine signaling via P2X7 receptor. Brain Behav. Immun. 50, 87-100. doi: 10.1016/j.bbi.2015.06.020

Xu, X., Zheng, S., Xiong, Y., Wang, X., Qin, W., Zhang, H., et al. (2017). Adenosine effectively restores endotoxin-induced inhibition of human neutrophil chemotaxis via A1 receptor-p38 pathway. Inflamm. Res. 66, 353-364. doi: 10.1007/s00011-016-1021-3

Yamada, K., Kobayashi, M., and Kanda, T. (2014). Involvement of adenosine A2A receptors in depression and anxiety. Int. Rev. Neurobiol. 119, 373-393. doi: 10.1016/B978-0-12-801022-8.00015-5

Yamauchi, T., and Kadowaki, T. (2013). Adiponectin receptor as a key player in healthy longevity and obesity-related diseases. Cell Metab. 17, 185-196. doi: 10.1016/j.cmet.2013.01.001

Yan, D., Zhu, Y., Walsh, T., Xie, D., Yuan, H., Sirmaci, A., et al. (2013). Mutation of the ATP-gated $\mathrm{P} 2 \mathrm{X} 2$ receptor leads to progressive hearing loss and increased susceptibility to noise. Proc. Natl. Acad. Sci. U.S.A. 110, 2228-2233. doi: 10.1073/pnas.1222285110

Yan, H., Zhang, E., Feng, C., and Zhao, X. (2016). Role of A3 adenosine receptor in diabetic neuropathy. J. Neurosci. Res. 94, 936-946. doi: 10.1002/jnr.23774

Yan, S., Du, F., Wu, L., Zhang, Z., Zhong, C., Yu, Q., et al. (2016). F1F0 ATP synthase-cyclophilin D interaction contributes to diabetes-induced synaptic dysfunction and cognitive decline. Diabetes Metab. Res. Rev. 65, 3482-3494.

Yan, Y., Bai, J., Zhou, X., Tang, J., Jiang, C., Tolbert, E., et al. (2015). P2X7 receptor inhibition protects against ischemic acute kidney injury in mice. Am. J. Physiol. Cell Physiol. 308, C463-C472. doi: 10.1152/ajpcell.00245.2014 
Yang, C., Xu, H., Cai, L., Du, X., Jiang, Y., Zhang, Y., et al. (2016). Donor pretreatment with adenosine monophosphate-activated protein kinase activator protects cardiac grafts from cold ischaemia/reperfusion injury. Eur. J Cardiothorac. Surg. 49, 1354-1360. doi: 10.1093/ejcts/ezv413

Yang, Z., Li, L., Zheng, J., Ma, H., Tian, S., Li, J., et al. (2016). Identification of a new series of potent adenosine A2A receptor antagonists based on 4-amino-5carbonitrile pyrimidine template for the treatment of Parkinson's disease. ACS Chem. Neurosci. 7, 1575-1584. doi: 10.1021/acschemneuro.6b00218

Yang, P., Wang, Z., Zhan, Y., Wang, T., Zhou, M., Xia, L., et al. (2013). Endogenous A1 adenosine receptor protects mice from acute ethanol-induced hepatotoxicity. Toxicology 309, 100-106. doi: 10.1016/j.tox.2013.05.003

Yap, S. C., and Lee, H. T. (2012). Adenosine and protection from acute kidney injury. Curr Opin. Nephrol. Hypertens 21, 24-32. doi: 10.1097/MNH. 0b013e32834d2ec 9

Yegutkin, G. G., Guerrero-Toro, C., Kilinc, E., Koroleva, K., Ishchenko, Y., Abushik, P., et al. (2016). Nucleotide homeostasis and purinergic nociceptive signaling in rat meninges in migraine-like conditions. Purinergic Signal. 12, 561-574. doi: 10.1007/s11302-016-9521-8

Yegutkin, G. G., Marttila-Ichihara, F., Karikoski, M., Niemela, J., Laurila, J. P., Elima, K., et al. (2011). Altered purinergic signaling in CD73-deficient mice inhibits tumor progression. Eur. J. Immunol. 41, 1231-1241. doi: 10.1002/eji. 201041292

Yelovitch, S., Barr, H. M., Camden, J., Weisman, G. A., Shai, E., Varon, D., et al. (2012). Identification of a promising drug candidate for the treatment of type 2 diabetes based on a P2Y1 receptor agonist. J. Med. Chem. 55, 7623-7635. doi: $10.1021 / \mathrm{jm} 3006355$

Yetgin, T., Uitterdijk, A., Te Lintel Hekkert, M., Merkus, D., KrabbendamPeters, I., Van Beusekom, H. M., et al. (2015). Limitation of infarct size and no-reflow by intracoronary adenosine depends critically on dose and duration. JACC Cardiovasc. Interv. 8, 1990-1999. doi: 10.1016/j.jcin.2015.08.033

Yip, L., Taylor, C., Whiting, C. C., and Fathman, C. G. (2013). Diminished adenosine A1 receptor expression in pancreatic $\alpha$-cells may contribute to the pathology of type 1 diabetes. Diabetes Metab. Res. Rev. 62, 4208-4219. doi: $10.2337 / \mathrm{db} 13-0614$

Yoneyama, Y., Sawa, R., Suzuki, S., Shin, S., Power, G. G., and Araki, T. (1996). The relationship between uterine artery Doppler velocimetry and umbilical venous adenosine levels in pregnancies complicated by preeclampsia. Am. J. Obstet. Gynecol. 174, 267-271. doi: 10.1016/S0002-9378(96)70406-4

Yoneyama, Y., Suzuki, S., Sawa, R., and Araki, T. (2004). Plasma adenosine concentrations increase in women with hyperemesis gravidarum. Clin. Chim. Acta 342, 99-103. doi: 10.1016/j.cccn.2003.12.004

Yoshida, K., Ito, M., and Matsuoka, I. (2015). P2X7 receptor antagonist activity of the anti-allergic agent oxatomide. Eur. J. Pharmacol. 767, 41-51. doi: 10.1016/j. ejphar.2015.10.002

Young, C. N. J., Sinadinos, A., and Gorecki, D. C. (2013). P2X receptor signaling in skeletal muscle health and disease. Wiley Interdiscip. Rev. Membr. Transp. Signal. 2, 265-274. doi: 10.1042/BJ20141551

Yu, Q., Guo, Z., Liu, X., Ouyang, Q., He, C., Burnstock, G., et al. (2013). Block of $\mathrm{P} 2 \mathrm{X} 7$ receptors could partly reverse the delayed neuronal death in area CA1 of the hippocampus after transient global cerebral ischemia. Purinergic Signal. 9, 663-675. doi: 10.1007/s11302-013-9379-y

Yu, W., Sun, X., Robson, S. C., and Hill, W. G. (2013). Extracellular UDP enhances $\mathrm{P} 2 \mathrm{X}$-mediated bladder smooth muscle contractility via P2Y6 activation of the phospholipase C/inositol trisphosphate pathway. FASEB J. 27, 1895-1903. doi: 10.1096/fj.12-219006

Yu, Y. (2015). Nucleotide modulates odor response through activation of purinergic receptor in olfactory sensory neuron. Biochem. Biophys. Res. Commun. 463, 1006-1011. doi: 10.1016/j.bbrc.2015.06.050

Yu, Y., and Zhang, C. (2014). Purinergic signaling negatively regulates activity of an olfactory receptor in an odorant-dependent manner. Neuroscience 275, 89-101. doi: 10.1016/j.neuroscience.2014.05.055

Yu, Y. C., Sohma, Y., and Hwang, T. C. (2016). On the mechanism of gating defects caused by the $\mathrm{R} 117 \mathrm{H}$ mutation in cystic fibrosis transmembrane conductance regulator. J. Physiol. 594, 3227-3244. doi: 10.1113/JP271723

Yuryeva, K., Saltykova, I., Ogorodova, L., Kirillova, N., Kulikov, E., Korotkaya, E., et al. (2015). Expression of adenosine receptors in monocytes from patients with bronchial asthma. Biochem. Biophys. Res. Commun. 464, 1314-1320. doi: 10.1016/j.bbrc.2015.07.141
Zafrah, H. A., and Alotaibi, M. F. (2017). The effect of extracellular ATP on rat uterine contraction from different gestational stages and its possible mechanisms of action. J. Basic Clin. Physiol. Pharmacol. 28, 209-217. doi: 10.1515/jbcpp-2016-0118

Zakeri, Z., Izadi, S., Niazi, A., Bari, Z., Zendeboodi, S., Shakiba, M., et al. (2012). Comparison of adenosine deaminase levels in serum and synovial fluid between patients with rheumatoid arthritis and osteoarthritis. Int. J. Clin. Exp. Med. 5, 195-200.

Zanin, R. F., Da Silva, G. L., Erig, T., Sperotto, N. D., Leite, C. E., CoutinhoSilva, R., et al. (2015). Decrease of serum adenine nucleotide hydrolysis in an irritant contact dermatitis mice model: potential P2X7R involvement. Mol. Cell. Biochem. 404, 221-228. doi: 10.1007/s11010-015-2381-7

Zarjou, A., and Agarwal, A. (2011). ATP as a death factor: purinergic signaling in renal epithelial-fibroblast cross talk. Am. J. Physiol. Renal Physiol. 300, F60-F61. doi: 10.1152/ajprenal.00593.2010

Zempo, H., Sugita, Y., Ogawa, M., Watanabe, R., Suzuki, J., and Isobe, M. (2015). A P2X7 receptor antagonist attenuates experimental autoimmune myocarditis via suppressed myocardial $\mathrm{CD} 4+\mathrm{T}$ and macrophage infiltration and $\mathrm{NADPH}$ oxidase 2/4 expression in mice. Heart Vessels 30, 527-533. doi: 10.1007/s00380014-0527-2

Zhang, B., Cheng, B., Li, F. S., Ding, J. H., Feng, Y. Y., Zhuo, G. Z., et al. (2015a). High expression of CD39/ENTPD1 in malignant epithelial cells of human rectal adenocarcinoma. Tumour. Biol. 36, 9411-9419. doi: 10.1007/s13277-0153683-9

Zhang, C., Gao, L., Yi, Y., Han, H., Cheng, H., Ye, X., et al. (2016). Adenosine triphosphate regresses endometrial explants in a rat model of endometriosis. Reprod. Sci. 23, 924-930. doi: 10.1177/1933719115625847

Zhang, K., Liu, J., You, X., Kong, P., Song, Y., Cao, L., et al. (2016). P2X7 as a new target for chrysophanol to treat lipopolysaccharide-induced depression in mice. Neurosci. Lett. 613, 60-65. doi: 10.1016/j.neulet.2015.12.043

Zhang, X., Qin, J., Zou, J., Lv, Z., Tan, B., Shi, J., et al. (2016). Extracellular ADP facilitates monocyte recruitment in bacterial infection via ERK signaling. Cell Mol. Immunol. doi: 10.1038/cmi.2016.56 [Epub ahead of print].

Zhang, Y., Guo, Q., Li, X., Gao, J., Liu, Y., Yang, J., et al. (2015b). P2Y purinergic receptor-regulated insulin secretion is mediated by a cAMP/Epac/Kv channel pathway. Biochem. Biophys. Res. Commun. 460, 850-856. doi: 10.1016/j.bbrc. 2015.03.121

Zhang, Y., Peti-Peterdi, J., Müller, C. E., Carlson, N. G., Baqi, Y., Strasburg, D. L., et al. (2015c). P2Y12 receptor localizes in the renal collecting duct and its blockade augments arginine vasopressin action and alleviates nephrogenic diabetes insipidus. J. Am. Soc. Nephrol. 26, 2978-2987. doi: 10.1681/ASN. 2014010118

Zhang, Y., Yuan, F., Cao, X., Zhai, Z., Ganghuang, Du, X., et al. (2014). P2X7 receptor blockade protects against cisplatin-induced nephrotoxicity in mice by decreasing the activities of inflammasome components, oxidative stress and caspase-3. Toxicol. Appl. Pharmacol. 281, 1-10. doi: 10.1016/j.taap.2014. 09.016

Zhao, H., Zhang, X., Dai, Z., Feng, Y., Li, Q., Zhang, J. H., et al. (2016). P2X7 receptor suppression preserves blood-brain barrier through inhibiting RhoA activation after experimental intracerebral hemorrhage in rats. Sci. Rep. 6:23286. doi: 10.1038/srep23286

Zhao, J., Wang, H., Dai, C., Wang, H., Zhang, H., Huang, Y., et al. (2013). P2X7 blockade attenuates lupus nephritis by inhibiting NLRP3/ASC/caspase-1 activation. Arthritis Rheum. 65, 3176-3185. doi: 10.1002/art.38174

Zhao, R., Liang, D., and Sun, D. (2016). Blockade of extracellular ATP effect by oxidized ATP effectively mitigated induced mouse experimental autoimmune uveitis (EAU). PLOS ONE 11:e0155953. doi: 10.1371/journal.pone.015 5953

Zheng, B., Lai, R., Li, J., and Zuo, Z. (2017). Critical role of P2X7 receptors in the neuroinflammation and cognitive dysfunction after surgery. Brain Behav. Immun. 61, 365-374. doi: 10.1016/j.bbi.2017.01.005

Zheng, X., Li, T., Chen, Y., Pan, H., Zhang, Z., Dai, Y., et al. (2017). Genetic polymorphisms of the $\mathrm{P} 2 \mathrm{X} 7$ gene associated with susceptibility to and prognosis of pulmonary tuberculosis. Infect. Genet. Evol. 53, 24-29. doi: 10.1016/j.meegid. 2017.05.003

Zhong, H., Yang, L., Belardinelli, L., and Zeng, D. (2007). Pro-fibrotic roles of the A2B adenosine receptor in human primary hepatic stellate cells. J. Hepatol. 46, S135-S135. doi: 10.1016/S0168-8278(07)61943-6 
Zhong, Y., Yang, Z., Huang, W. C., and Luo, X. (2013). Adenosine, adenosine receptors and glaucoma: an updated overview. Biochim. Biophys. Acta 1830, 2882-2890. doi: 10.1016/j.bbagen.2013.01.005

Zhou, Q., Xu, C., Cheng, X., Liu, Y., Yue, M., Hu, M., et al. (2016). Platelets promote cartilage repair and chondrocyte proliferation via ADP in a rodent model of osteoarthritis. Platelets 27, 212-222. doi: 10.3109/09537104.2015.107 5493

Zhou, X., Ma, L. M., Xiong, Y., Huang, H., Yuan, J. X., Li, R. H., et al. (2016). Upregulated P2X3 receptor expression in patients with intractable temporal lobe epilepsy and in a rat model of epilepsy. Neurochem. Res. 41, 1263-1273. doi: 10.1007/s11064-015-1820-x

Zhu, J., Zeng, Y., Li, W., Qin, H., Lei, Z., Shen, D., et al. (2017). CD73/NT5E is a target of miR-30a-5p and plays an important role in the pathogenesis of non-small cell lung cancer. Mol. Cancer 16:34. doi: 10.1186/s12943-017-0591-1

Zhu, Y., and Zhao, H. B. (2012). ATP activates P2X receptors to mediate gap junctional coupling in the cochlea. Biochem. Biophys. Res. Commun. 426, 528-532. doi: 10.1016/j.bbrc.2012.08.119

Zhu, Y. J., and Lu, T. J. (2010). A multi-scale view of skin thermal pain: from nociception to pain sensation. Philos. Trans. A Math. Phys. Eng. Sci. 368, 521-559. doi: 10.1098/rsta.2009.0234
Zierhut, M., Dyckhoff, S., Masouris, I., Klein, M., Hammerschmidt, S., Pfister, H. W., et al. (2017). Role of purinergic signaling in experimental pneumococcal meningitis. Sci. Rep. 7:44625. doi: 10.1038/srep44625

Ziganshin, A. U., Vafina, Z. R., and Fatkullin, I. F. (2008). Contrasting effects of P2 receptor agonists on spontaneous contractility of human fallopian tubes with and without acute inflammation. Pharmacol. Res. 57, 56-59. doi: 10.1016/j.phrs. 2007.11.004

Conflict of Interest Statement: The author declares that the research was conducted in the absence of any commercial or financial relationships that could be construed as a potential conflict of interest.

The reviewer SS and handling Editor declared their shared affiliation.

Copyright (C) 2017 Burnstock. This is an open-access article distributed under the terms of the Creative Commons Attribution License (CC BY). The use, distribution or reproduction in other forums is permitted, provided the original author(s) or licensor are credited and that the original publication in this journal is cited, in accordance with accepted academic practice. No use, distribution or reproduction is permitted which does not comply with these terms. 